SLAC-R-545

UC-414

\title{
A Test of the Flavor Independence of
}

Strong Interactions in $\mathrm{e}^{+} \mathrm{e}^{-}$Annihilation at the $\mathrm{Z}^{0}$ Pole

\author{
Noritsugu Oishi \\ Stanford Linear Accelerator Center \\ Stanford University \\ Stanford, CA 94309
}

SLAC-Report-545

September 1999

\section{Prepared for the Department of Energy \\ under contract number DE-AC03-76SF00515}

Printed in the United States of America. Available from the National Technical Information Service, U.S. Department of Commerce, 5285 Port Royal Road, Springfield, VA 22161.

* Ph.D. thesis, Nagoya University, Department of Physics, Nagoya 464-8602, Japan. 
DPNU-98-31

SLAC-R-545

\section{A TEST OF THE FLAVOR INDEPENDENCE OF STRONG INTERACTIONS IN $\mathrm{e}^{+} \mathrm{e}^{-}$ ANNIHILATION AT THE $Z^{0}$ POLE}

NORITSUGU OISHI

Department of Physics, Nagoya University Nagoya 464-8602, Japan

July 1998

Ph.D Dissertation

\section{Abstract}

This thesis presents a comparison of the strong coupling of the gluons to light $\left(q_{l}=\right.$ $u+d+s), c$, and $b$ quarks, determined from multijet rates in flavor-tagged samples of approximately 150,000 hadronic $Z^{0}$ decays recorded with the SLC Large Detector at the SLAC Linear Collider between 1993 and 1995.

Flavor separation among primary $q_{l} \bar{q}_{l}, c \bar{c}$ and $b \bar{b}$ final states was made on the basis of the reconstructed mass of long-lived heavy-hadron decay vertices, yielding tags with high purity and low bias against $\geq 3$-jet final states.

We find:

$$
\begin{aligned}
\alpha_{s}^{\text {uds }} / \alpha_{s}^{\text {all }} & =0.987 \pm 0.010(\text { stat } .)_{-0.010}^{+0.012}(\text { syst. })_{-0.008}^{+0.009}(\text { theor } y) \\
\alpha_{s}^{c} / \alpha_{s}^{\text {all }} & =1.023 \pm 0.034(\text { stat } .)_{-0.036}^{+0.032}(\text { syst. })_{-0.014}^{+0.018}(\text { theor } y) \\
\alpha_{s}^{b} / \alpha_{s}^{\text {all }} & =0.993 \pm 0.016(\text { stat } .)_{-0.023}^{+0.020}(\text { syst } .)_{-0.027}^{+0.019}(\text { theory })
\end{aligned}
$$

and alternatively

$$
\alpha_{s}^{c} / \alpha_{s}^{u d s}=1.036 \pm 0.043(\text { stat. })_{-0.045}^{+0.041}(\text { syst. })_{-0.018}^{+0.020}(\text { theory })
$$$$
\alpha_{s}^{b} / \alpha_{s}^{u d s}=1.004 \pm 0.018(\text { stat. })_{-0.031}^{+0.026}(\text { syst. })_{-0.029}^{+0.018}(\text { theory })
$$

which implies that no flavor dependence within our sensitivity. 


\section{Acknowledgments}

I would like to appreciate Prof. T. Ohshima, S. Suzuki, A. Sugiyama, and Prof. R. Kajikawa of the high energy physics laboratory $(N$-lab.) at Nagoya University for their continuous assistance.

I am extremely thankful to P. N. Burrows, D. Muller for their brilliant suggestions and patients. I would particularly like to thank T. Maruyama, P. Dervan, D. Dong, T. Abe with whom I had useful discussions every week, I also thank all of the SLD QCD working group members who give me countless suggestions, and D. Jackson, J. Yashima, E. Weiss, for their numerous suggestions about the flavor tagging technique. M. Hildreth who provided me the basic ideas of unfolding formulae, D. Su for his patients to answer my questions about heavy flavor physics. I also thank A. Brandenburg, P. Uwer and L. Dixon for calculating the QCD coefficients used in the theoretical translations and for their assistance with this analysis, as well as T. Rizzo for many useful discussions.

I would like to thank the SLD Collaboration of the Stanford Linear Accelerator and for the opportunity of engaging in this research, and the personnel of the SLAC accelerato department and the technical staffs of our collaborating institutions for their outstanding efforts on our behalf. I owe a tremendous debt to my friends in the United States of America for their hospitality. Finally, I express my best gratitude to my parents for their support.

This work was supported by the U.S. Department of Energy and National Science Foundation, the UK Particle Physics and Astronomy Research Council, the Istituto Nazionale di Fisica Nucleare of Italy, the Japan-US Cooperative Research Project on High Energy Physics, and the Korea Research Foundation.

\section{Contents}

1 Introduction

2 The Strong Interaction in $e^{+} e^{-}$Annihilation 4

2.1 Quarks and Interactions . . . . . . . . . . . . . . . . . . . 4

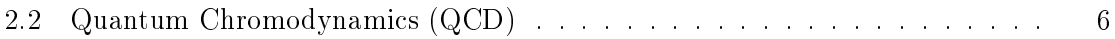
2.2.1 Strong Coupling and Perturbative QCD . . . . . . . . . 8 2.2.2 Unitarity and Flavor Independence of QCD . . . . . . . . . . . . 11

2.3 Hadron Production in $e^{+} e^{-}$Collisions . . . . . . . . . . . . . . . . . . . . . 14 $2.3 .1 e^{+} e^{-}$annihilation into hadrons $\ldots \ldots \ldots \ldots \ldots$

2.3 .2 Parton Production . . . . . . . . . . . . . . . 18

2.3.3 Hadronization . . . . . . . . . . . . . . . . . . . . . . . . . 24

2.4 Current Experimental Status . . . . . . . . . . . . . . . . . . . . . . 28

2.4 .1 Measurements of $\alpha_{s}$ ratios . . . . . . . . . . . . . . . 28

2.4.2 Anomalous Chromomagnetic Moment . . . . . . . . . . . . . . . 31

\section{Experimental Apparatus}

3.1 The SLAC Linear Collider $(\mathrm{SLC}) \ldots \ldots \ldots$. . . . . . . . . . . 34

3.2 SLC Large Detector $(\mathrm{SLD}) \ldots \ldots \ldots \ldots$

3.2 .1 Vertex Detector $($ VXD2) $\ldots \ldots \ldots \ldots$

3.2 .2 Central Drift Chamber $(\mathrm{CDC}) \ldots \ldots \ldots \ldots \ldots$. . . . . . . 40

3.2.3 Cherenkov Ring Imaging Detector (CRID) . . . . . . . . . . . . 43

3.2.4 Liquid Argon Calorimeter(LAC) . . . . . . . . . . . . . . . . . . . . 44

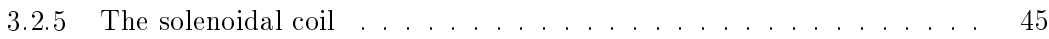

3.2.6 Warm Iron Calorimeter (WIC) $\ldots \ldots \ldots \ldots \ldots$. . . . . . . . 45 
3.2 .7 Luminosity Monitor (LUM) . . . . . . . . . . . . . . . . . . . 46

3.3 The SLD Monte Carlo Simulation . . . . . . . . . . . . . . . . . . . . . . 47

3.3 .1 Physics Simulation . . . . . . . . . . . . . . . . . . 47

3.3.2 Detector Simulation . . . . . . . . . . . . . . . 48

3.4 Tracking System Performance . . . . . . . . . . . . . . . . . . . . . . . 49

3.4.1 Charged Track Reconstruction . . . . . . . . . . . . . . . . . . 49

3.4 .2 Impact Parameter Resolution . . . . . . . . . . . . . . . . 51

3.4.3 Determination of the Interaction Point (IP) . . . . . . . . . . 51

4 Event Selection and Flavor Tagging $\quad \mathbf{5 6}$

4.1 Hadronic Event Selection . . . . . . . . . . . . . . . . . . . . . . . . . 57

4.1 .1 Event Trigger . . . . . . . . . . . . . . . . . . . . 57

4.1 .2 Hadronic Event Filter . . . . . . . . . . . . . . . . 60

4.1 .3 Event Selection . . . . . . . . . . . . . . . . . 60

4.2 Flavor Tagging . . . . . . . . . . . . . . . . . . . . . . 64

4.2.1 Flavor Tagging Quality Track Selection . . . . . . . . . . . . . . 64

4.2 .2 Topological Vertex Mass . . . . . . . . . . . . . . . . . 68

4.2 .3 Requirements for Flavor Tags . . . . . . . . . . . . . 71

$5 \quad$ Measurement of the $\alpha_{s}$ (flavor) ratios $\quad 78$

5.1 Introduction . . . . . . . . . . . . . . . . . . . . . . 78

5.2 Jet Finding . . . . . . . . . . . . . . . . . . . . . . 79

5.2 .1 Jet Clustering Algorithms . . . . . . . . . . . . . . . . 79

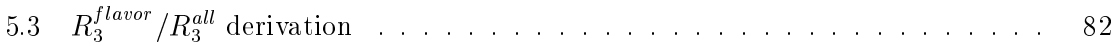

5.3 .1 Detector level jet rate . . . . . . . . . . . . . . . . . . . 82

5.3.2 Parton level $R_{3}$ Unfolding . . . . . . . . . . . . . . . . . . 84

$5.3 .3 R_{3}^{i} / R_{3}^{\text {all }}$ and Propagation of Errors . . . . . . . . . . . 85

5.4 Systematic Errors on $R_{3}^{i} / R_{3}^{\text {all }} \ldots \ldots \ldots \ldots \ldots \ldots$

5.4 .1 Detector Modeling . . . . . . . . . . . . . . . . . . . 96

5.4 .2 MC statistics . . . . . . . . . . . . . . . . . . . . . . 102

5.4 .3 Physics Modeling . . . . . . . . . . . . . . . . . . . . . 102

5.4.4 Hadronization Uncertainties . . . . . . . . . . . . . . . . . . 105
CONTENTS

5.4 .5 Choice of $y_{c u t}$ Values . . . . . . . . . . . . . . . . . . 113

5.5 Translation to $\alpha_{s}$ Ratios . . . . . . . . . . . . . . . . . . . . . 120

5.5.1 Heavy Quark Mass Effects . . . . . . . . . . . . . . . . . . . . 120

5.5.2 Derivation of $\alpha_{s}^{\text {uds }} / \alpha_{s}^{\text {all }}, \alpha_{s}^{c} / \alpha_{s}^{\text {all }}$, and $\alpha_{s}^{b} / \alpha_{s}^{\text {all }} \ldots \ldots \ldots \ldots 124$

5.5.3 Translation Uncertainties . . . . . . . . . . . . . . . . . . . . . . 128

5.5 .4 Comparison of $\alpha_{s}$ Ratios . . . . . . . . . . . . . . . . . . . . 133

5.5.5 Alternative ratios $\alpha_{s}^{c} / \alpha_{s}^{u d s}$ and $\alpha_{s}^{b} / \alpha_{s}^{u d s} \ldots \ldots \ldots \ldots \ldots$

5.6 Cross Checks . . . . . . . . . . . . . . . . . . . 140

5.6 .1 Variation of Topological Vertex Tagging . . . . . . . . . . . . . 140

5.6 .2 Lifetime-based Tag . . . . . . . . . . . . . . . . . . . . . . . . . . . . 142

5.6 .3 Discussion . . . . . . . . . . . . . . . . . . . . . . . . . . . 148

6 Summary, Discussion and Conclusions 150

Appendix

A $V^{0}$ Rejection

B Unfolding Scheme using the Least Squares Method

C Possible Improvements with the VXD3 detector

D The SLD collabolation

References 


\section{LIST OF TABLES}

5.13 The coefficient $A^{b}, B^{b}, C^{b}$ for the massive next-to-leading-order calculation. 123

5.14 Correlations between flavors . . . . . . . . . . . . . . . . . . . 128

5.15 Leading and next-to-leading order contributions to the 4 -jet rate. . . . . . . 130

5.16 Renormalization scale and corresponding $\alpha_{s}$ ranges used to estimate higherorder perturbative QCD uncertainties. . . . . . . . . . . . . . . . 131

\section{List of Tables}

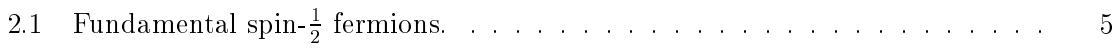

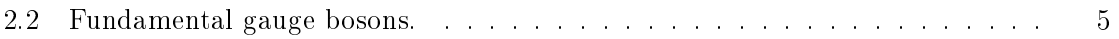

2.3 The vector and axial vector couplings for fermions to the $Z^{0}$ gauge boson. . . 17

2.4 Some properties of the $Z^{0} \ldots \ldots \ldots \ldots \ldots \ldots$

3.1 JETSET7.4 parameters: default and optimized values . . . . . . . . . . . . . 47

4.1 The efficiencies for hadronic events of different primary quark flavors to pass the event selection cuts. . . . . . . . . . . . . . . . . 62

4.2 Summary of flavor-tagged subsamples . . . . . . . . . . . . . . . 74

5.1 Jet finging algorithm . . . . . . . . . . . . . . . . . . 81

5.2 The number of 2- and $\geq 3$-jet events for each subsample and algorithm. . . . 83

5.3 Results for $R_{3}^{j} / R_{3}^{\text {all }}$, derived from Eq. 5.11; The errors shown are statistical. 86

5.4 Smearing factors $C\left(P_{t}, \cos \theta\right)$ for 2-dimensional normalized impact parameter

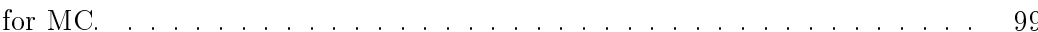

5.5 Compilation of the systematic errors for the E algorithm and $y_{c u t}=0.04$. . 107

5.6 Compilation of the systematic errors for the E0 algorithm and $y_{\text {cut }}=0.02$. 108

5.7 Compilation of the systematic errors for the P algorithm and $y_{c u t}=0.02 . \quad$. 109

5.8 Compilation of the systematic errors for the P0 algorithm and $y_{\text {cut }}=0.015$. 110

5.9 Compilation of the systematic errors for the Durham algorithm and $y_{\text {cut }}=0.01 .111$

5.10 Compilation of the systematic errors for the Geneva algorithm and $y_{\text {cut }}=0.08 .112$

5.11 Central values and errors on $R_{3}^{i} / R_{3}^{\text {all }}$ for the 6 different jet-finding algorithms

at the respective chosen $y_{c}$ values. . . . . . . . . . . . . 113

5.12 Massless coefficients $A^{0}, B^{0}$, and $C^{0}$ for each algorithm. . . . . . . . . . 123
5.17 Summary of translation uncertainties on the $\alpha_{s}$ ratios for each algorithm. . . 132

5.18 Central values of $\alpha_{s}^{i} / \alpha_{s}^{\text {all }}$ and errors. . . . . . . . . . . . 134

5.19 Compilation of the systematic errors for the E0 algorithm and $y_{c u t}=0.02 . \quad$. 136

5.20 Summary of translation uncertainties on $\alpha_{s}^{i} / \alpha_{s}^{u d s}$ for each algorithm . . . . 137

$5.21 R_{3}^{i} / R_{3}^{u d s}$ and $\alpha_{s}^{i} / \alpha_{s}^{u d s}$ values and errors. . . . . . . . . . . . . . 138

5.22 Results of $R_{3}^{i} / R_{3}^{\text {all }}(i=u d s, c, b)$ using lifetime (impact parameter) tag. The errors shown are statistical only. . . . . . . . . . . . . . . . 146

5.23 Systematic errors on the results of $\alpha_{s}^{i} / \alpha_{s}^{\text {all }}$ using lifetime tag. The first column shows the error source, the second column the central value used, and the third column the variation considered. The remaining columns show the corresponding errors on the values of $\alpha_{s}^{i} / \alpha_{s}^{\text {all }}(i=u d s, c, b) \ldots \ldots \ldots \ldots$

5.24 (a) Comparison of tagging methods . . . . . . . . . . . . . . . . 148

C.1 VXD3/VXD2 Comparison. . . . . . . . . . . . . . . . . . . . . . . 160

C.2 VXD3/VXD2 comparison for flavor tagging performance . . . . . . . . 162 
3.6 Wire configuration of the Central Drift Chamber (CDC). . . . . . . . . . . 42

3.7 Schematic view of the Cherenkov Ring Imaging Detector(CRID) . . . . . . . 43

3.8 LAC barrel EM and HAD modules . . . . . . . . . . . . . . . . . . . . . . . . . . . . . . 44

3.9 Warm Iron Calorimeter . . . . . . . . . . . . . . . . . . 46

3.10 A side view of the Luminosity Monitor (LUM). . . . . . . . . . . . . . 46

\section{List of Figures}

2.1 (a) quark-gluon, (b) triple-gluon, (c) four-gluon coupling. . . . . . . . . . 8

2.2 The values of $\alpha_{s}(Q)$ at the values of $Q$ where they are measured. . . . . . 9

2.3 Determination of absolute value of $\alpha_{s} \ldots \ldots \ldots \ldots \ldots$

2.4 Summary of world $\alpha_{s}\left(M_{Z}^{2}\right)$ measurements. . . . . . . . . . . . . . . 10

2.5 Feynman diagrams of (a) the bare $q \bar{q} g$ vertex; (b) the vertex corrections; (c) the quark self-energy corrections; (d) the corrections to the gluon propagator, and the corresponding vertex factors. . . . . . . . . . . . . . 13

2.6 Schematic view of the renormalized couplings of $q \bar{q} g$ (a), and $g g g(\mathrm{~b}) \ldots \ldots$

2.7 The phases in the process of hadronic production in $e^{+} e^{-}$annihilation. . . . 15

2.8 Total cross section for $e^{+} e^{-}$annihilation into hadrons and muon pairs as function of the center-of-mass energy. . . . . . . . . . . . 17

2.9 Feynman diagrams for $e^{+} e^{-} \rightarrow q \bar{q}$, (a) $\mathcal{O}\left(\alpha_{s}^{0}\right)$, (b) $\mathcal{O}\left(\alpha_{s}^{1}\right)$, and (c) $\mathcal{O}\left(\alpha_{s}^{2}\right) . \quad \ldots \quad 19$

2.10 Feynman diagrams for $e^{+} e^{-} \rightarrow q \bar{q} g$, (a) $\mathcal{O}\left(\alpha_{s}\right)$ and (b) $\mathcal{O}\left(\alpha_{s}^{2}\right) \ldots \ldots . \ldots 20$

2.11 Feynman diagrams for four-jet events. . . . . . . . . . . . . . . 21

2.12 Schematic views of $q \bar{q} g$ string and hadronization in string model. . . . . . . . 24

2.13 Schematic of hadronization in cluster fragmentation scenario. . . . . . . . . . 26

2.14 Values of $\alpha_{s}^{\text {flavors }} / \alpha_{s}^{\text {all }}$ previously measured values. $\ldots \ldots \ldots 33$

3.1 The layout of the SLAC Linear Collider. . . . . . . . . . . . . . . 35

3.2 The history of the integrated luminosity for the 1991 through 1995 runs of the SLD experiment. . . . . . . . . . . . . . . . . . 36

3.3 The cutaway and quadrant sections of the SLD . . . . . . . . . . . . . 37

3.4 The SLD Vertex Detector, bird's-eye view (left) and $x y$ view (right). . . . . 38

3.5 Drift distance resolution as a function of the drift distance in a cell. . . . . . 41
3.11 Distribution of charge over momentum for tracks from $Z^{0} \rightarrow \mu^{+} \mu^{-}$decays. $\quad 50$

3.12 The impact parameter resolutions as a function of track momentum, for the

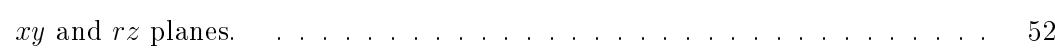

$3.13 \mu$-pair miss distance in $r \phi$ and $r z$ projections . . . . . . . . . . . . . . 52

3.14 (a) schematic view of the transverse IP position, (b) distribution of the track impact parameters in $\mu^{+} \mu^{-}$events. . . . . . . . . . . . . . 54

4.1 Distributions of the event selection variables for data (points) and MC hadronic events (histograms). . . . . . . . . . . . . . . 63

4.2 Distributions of the variables upon which cuts are applied to select flavor tagging quality tracks. . . . . . . . . . . . . . 66

4.3 (a): Definition of the impact parameter. (b): signing scheme of the impact

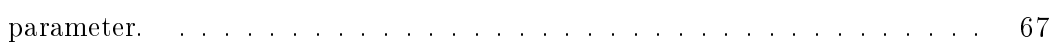

4.4 The normalized impact parameter distribution. . . . . . . . . . . . 67

4.5 Construction of the Gaussian tube $f_{i}(\mathbf{r})$ for each track $i$. . . . . . . . . . 69

4.6 (a) Track and $(b)$ vertex functions projected onto the $x y$ plane for a Monte Carlo event. . . . . . . . . . . . . . . . 6 69

4.7 Schematic description of missing transverse momentum, $P_{t}^{v}$, and vertex im-

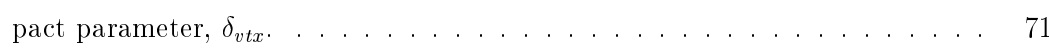

4.8 The distribution of the corrected vertex-mass $M_{v t x}$ and the vertex momentum $P_{v t x}$ in our data sample (points). . . . . . . . . . . . . 72

4.9 The two-dimensional distribution of vertex momentum $P_{v t x}$ vs. vertex mass

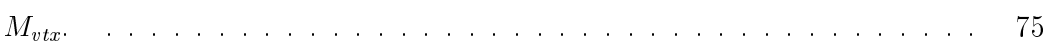

4.10 The distribution of vertex impact parameter, $\delta_{v t x}$ for events in region (B): data (points); the simulated distribution is shown as a histogram in which the contributions from events of different primary quark flavor are indicated . . . 76 


\section{LIST OF FIGURES}

4.11 The distribution of the number of tracks per event that miss the IP by at least $2 \sigma$ in terms of their impact parameter in the plane normal to the beamline, in events that contain no reconstructed vertex. . . . . . . . . . . 77

5.1 The detector-level n-jet rate vs. $y_{\text {cut }}$. The points are Data and lines are MC.

5.2 The detector-level $R_{3}^{j}$ vs. $y_{c u t}$ for each flavor-tagged subsample and for each algorithm. The points are Data and lines are MC. . . . . . . . . . . . 87

5.3 The unfolded parton-level $R_{3}$ for each flavor and different algorithms. The points are Data and lines are MC. Arrows indicate the chosen $y_{\text {cut }}$ values. .

5.4 (a) The raw measured ratios $R_{3}^{j} / R_{3}^{\text {all }}$ vs. $y_{\text {cut }}$ for subsample $j$. (b) The unfolded ratios $R_{3}^{i} / R_{3}^{\text {all }}, i=u d s, c, b$, vs. $y_{c}$ for the 3 primary event flavors. :

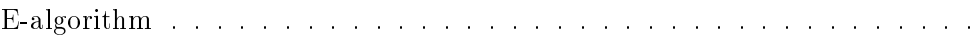

5.5 (a) The raw measured ratios $R_{3}^{j} / R_{3}^{\text {all }}$ vs. $y_{\text {cut }}$ for subsample $j$. (b) The unfolded ratios $R_{3}^{i} / R_{3}^{\text {all }}, i=u d s, c, b$, vs. $y_{c}$ for the 3 primary event flavors. :

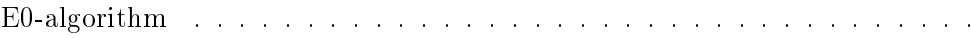

5.6 (a) The raw measured ratios $R_{3}^{j} / R_{3}^{\text {all }}$ vs. $y_{\text {cut }}$ for subsample $j$. (b) The unfolded ratios $R_{3}^{i} / R_{3}^{\text {all }}, i=u d s, c, b$, vs. $y_{c}$ for the 3 primary event flavors. :

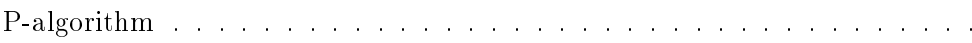

5.7 (a) The raw measured ratios $R_{3}^{j} / R_{3}^{\text {all }}$ vs. $y_{\text {cut }}$ for subsample $j$. (b) The unfolded ratios $R_{3}^{i} / R_{3}^{\text {all }}, i=u d s, c, b$, vs. $y_{c}$ for the 3 primary event flavors. :

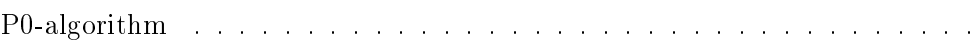

5.8 (a) The raw measured ratios $R_{3}^{j} / R_{3}^{\text {all }}$ vs. $y_{\text {cut }}$ for subsample $j$. (b) The unfolded ratios $R_{3}^{i} / R_{3}^{\text {all }}, i=u d s, c, b$, vs. $y_{c}$ for the 3 primary event flavors. :

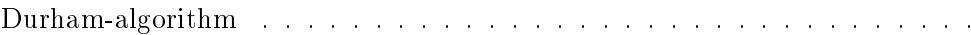

5.9 (a) The raw measured ratios $R_{3}^{j} / R_{3}^{\text {all }}$ vs. $y_{\text {cut }}$ for subsample $j$. (b) The unfolded ratios $R_{3}^{i} / R_{3}^{\text {all }}, i=u d s, c, b$, vs. $y_{c}$ for the 3 primary event flavors. :

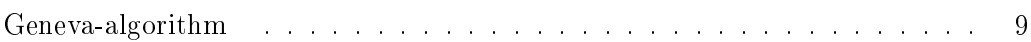

5.10 The flavor tag bias against 3-jet as a function of $y_{\text {cut }}$. Arrows indicate the

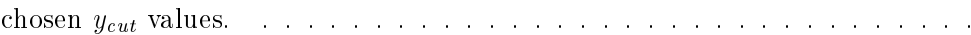

5.11 (a) Transverse momentum (w.r.t beam axis) distribution for the well-measured tracks, (b) Transverse momentum (w.r.t beam axis) distribution for the flavor tagging quality tracks, (c) Polar angle (w.r.t beam axis) distribution for the flavor tagging quality tracks, for data (points) and Monte Carlo (histogram), respectively. (d-f); Data/MC ratio for (a-c), respectively. . . . . . . . . . . . 97

5.12 Distribution of the normalized 2D impact parameter for data (points) and Monte Carlo (histogram) comparing before smearing (a) and after smearing $(\mathrm{c})$, and the corresponding ratios $(\mathrm{b})$ and $(\mathrm{d}) \ldots \ldots \ldots \ldots$

5.13 Distribution of the normalized 3D impact parameter for data (points) and Monte Carlo (histogram) comparing before smearing (a) and after smearing (c), and the corresponding ratios (b) and (d). . . . . . . . . . . . . . 101

5.14 Row(a) statistical errors; (b) experimental systematic errors; (c) hadronization uncertainties; (d) total errors on $R_{3}^{i} / R_{3}^{\text {all }}$ as a function of $y_{\text {cut }}$ for $i=u d s$ (first column), $i=$ charm (second column), and $i=b$ flavors (third column), respectively, for E-algorithm. Arrows indicate the chosen $y_{c u t}$ values. . . . . . 114

5.15 Row(a) statistical errors; (b) experimental systematic errors; (c) hadronization uncertainties; (d) total errors on $R_{3}^{i} / R_{3}^{\text {all }}$ as a function of $y_{\text {cut }}$ for $i=u d s$ (first column), $i=$ charm (second column), and $i=b$ flavors (third column), respectively, for E0-algorithm. Arrows indicate the chosen $y_{\text {cut }}$ values. . . . . 115

$5.16 \operatorname{Row}(\mathrm{a})$ statistical errors; (b) experimental systematic errors; (c) hadronization uncertainties; (d) total errors on $R_{3}^{i} / R_{3}^{\text {all }}$ as a function of $y_{c u t}$ for $i=u d s$ (first column), $i=$ charm (second column), and $i=b$ flavors (third column), respectively, for P-algorithm. Arrows indicate the chosen $y_{c u t}$ values. . . . . . 116

5.17 Row(a) statistical errors; (b) experimental systematic errors; (c) hadronization uncertainties; (d) total errors on $R_{3}^{i} / R_{3}^{\text {all }}$ as a function of $y_{\text {cut }}$ for $i=u d s$ (first column), $i=$ charm (second column), and $i=b$ flavors (third column), respectively, for P0-algorithm. Arrows indicate the chosen $y_{\text {cut }}$ values. . . . . 117

5.18 Row(a) statistical errors; (b) experimental systematic errors; (c) hadronization uncertainties; (d) total errors on $R_{3}^{i} / R_{3}^{\text {all }}$ as a function of $y_{\text {cut }}$ for $i=u d s$ (first column), $i=$ charm (second column), and $i=b$ flavors (third column), respectively, for Durham algorithm. Arrows indicate the chosen $y_{c u t}$ values. . 118 
5.19 Row(a) statistical errors; (b) experimental systematic errors; (c) hadronization uncertainties; (d) total errors on $R_{3}^{i} / R_{3}^{\text {all }}$ as a function of $y_{\text {cut }}$ for $i=u d s$ (first column), $i=$ charm (second column), and $i=b$ flavors (third column), respectively, for Geneva algorithm. Arrows indicate the chosen $y_{\text {cut }}$ values. . 119

5.20 The numerical calculation of the ratio; $R_{3}^{b} / R_{3}^{0}$ for the Durham algorithm as a function of $y_{c u t} \ldots \ldots \ldots \ldots \ldots \ldots \ldots$

5.21 Schematic view of the modification of jet rate for $b$ event. . . . . . . . . . 126

5.22 The measured ratios $R_{3}^{i} / R_{3}^{\text {all }}$ (a), and the corresponding translated ratios $\alpha_{s}^{i} / \alpha_{s}^{\text {all }}(i=u d s, c, b)(\mathrm{b})$. The arrows in (a) indicate the range of the theoretical prediction described in the text for values of the $b$-quark mass in the range $2.5 \leq m_{b}\left(M_{Z}\right) \leq 3.5 \mathrm{GeV} / c^{2}$, with the arrow pointing towards the lower mass value. In (b) the weighted average over the six algorithms is also shown. In all cases only statistical error bars are displayed. . . . . . . . . . . . 127

5.23 (a) $\alpha_{s}\left(M_{Z}^{2}\right)$ and (b) $\chi_{\text {dof }}^{2}$ from the $\mathcal{O}\left(\alpha_{s}^{2}\right)$ fits to the jet rates as a function of renormalization scale factor $f$ (see text) . . . . . . . . . . . . . 131

5.24 (a) The measured ratios $R_{3}^{i} / R_{3}^{u d s}$, and (b) the corresponding translated ratios $\alpha_{s}^{i} / \alpha_{s}^{u d s}(i=c, b)$. The arrows in (a) indicate the range of the theoretical prediction described in the text for values of the $b$-quark mass in the range $2.5 \leq m_{b}\left(M_{Z}\right) \leq 3.5 \mathrm{GeV} / c^{2}$, with the arrow pointing towards the lower mass value. In (b) the weighted average over the six algorithms is also shown. In all cases only statistical error bars are displayed. . . . . . . . . . . . 139

5.25 The distribution of the the number of tracks that miss the IP by at least $3 \sigma$ in terms of their impact parameter in the plane normal to the beamline. . . 143

5.26 (a) The raw measured ratios $R_{3}^{j} / R_{3}^{\text {all }}, 1 \leq j \leq 5$, vs. $y_{\text {cut }}$ for the 5 subsamples classified by $N_{\text {sig }}$ (see text); data (points with error bars), and simulation (lines joining values at the same $y_{\text {cut }}$ values as the data). (b) The unfolded $R_{3}^{i} / R_{3}^{\text {all }}, i=u d s, c, b$, vs. $y_{\text {cut }}$ for the 3 primary event flavor groups. Only statistical errors are shown. The arrow indicate the chosen $y_{\text {cut }}$ value. . . . . 144

5.27 The unfolded ratios $R_{3}^{i} / R_{3}^{\text {all }}, i=u d s, c, b$, vs. $y_{\text {cut }}$ for each algorithms using impact parameter tag. Only statistical errors are shown. Arrows indicate the chosen $y_{\text {cut }}$ values. . . . . . . . . . . . . . . . . . . . . . 145

5.28 (a) The measured ratios of $R_{3}^{i} / R_{3}^{\text {all }}$, and (b) translated ratios $\alpha_{s}^{i} / \alpha_{s}^{\text {all }}(i=$ $u d s, c, b)$ using lifetime (impact parameter) tag as a cross check (see text). The arrows in (a) indicate the range of the theoretical prediction described in the text for values of the $b$-quark mass in the range $2.5 \leq m_{b}\left(M_{Z}\right) \leq 3.5 \mathrm{GeV} / c^{2}$, with the arrow pointing towards the lower mass value. In (b) the weighted average over the six algorithms is also shown. In all cases only statistical error bars are displayed. . . . . . . . . . . . . . . . . . . . . 147

5.29 Summary of cross checks. . . . . . . . . . . . . . . . . . . . . 149

6.1 Summary of measurements of $\alpha_{s}^{i} / \alpha_{s}^{a l l}(i=u d s, c, b)$ from experiments at the $Z^{0}$ resonance. . . . . . . . . . . . . . . . . . . . . . 152

A.1 Distribution of the difference between reconstructed mass and expect value of the identified $\gamma$-conversion and $V^{0}$ candidates. The points represent the data and the histogram represent the Monte Carlo, whose hatched area is the fraction of random combinatorial background. . . . . . . . . . . . . . 155

A.2 Reconstructed gamma conversions as a function of distance from beam-line; the data (plots) and Monte Carlo(histogram). Peaks in the distribution correspond to the positions of the detector materials. . . . . . . . . . . . . . 156

C.1 Cross-section view ( $x y$ plane) of the VXD2 and VXD3. . . . . . . . . . . 161

C.2 Cross-section view ( $r z$ plane) of the VXD2 and VXD3. . . . . . . . . . 161

C.3 Comparison of impact parameter resolution as a function of track momentum for tracks at $\cos \theta=0$ for VXD2 and VXD3, where $\theta$ is the polar angle w.r.t the beam-axis. Points are measured data and lines are the Monte Carlo simulations. . . . . . . . . . . . . . . . . . 162 


\section{Chapter 1}

\section{Introduction}

The "Standard Model"; of elementary particles and fields, successfully predicts a large number of high energy physics phenomena. Some measurements in the electroweak sector $(S U(2) \times U(1))$ have reached a precision of better than $1 \%$ [1]. However, measurements of strong interactions $\left(S U(3)_{c}\right)$ have not yet achieved the same level of precision.

In order for Quantum Chromodynamics (QCD) to be a gauge-invariant renormalizable field theory it is required that the strong coupling $\alpha_{s}$ be independent of quark flavor. This basic ansatz can be tested directly in $e^{+} e^{-}$annihilation by measuring the strong coupling in events of the type $e^{+} e^{-} \rightarrow q \bar{q}(g)$ for specific quark flavors $q$.

Whereas an absolute determination of $\alpha_{s}$ using such a technique is limited, primarily by large theoretical uncertainties, to the 5\%-level of precision [2], a much more precise test of flavor-independence can be made from the ratio of the couplings for different quark flavors, in which most experimental errors and theoretical uncertainties are expected to cancel. Furthermore, it has recently been suggested [3] that the emission of gluon radiation in $b \bar{b}$ events is expected to be modified relative to that in $q_{l} \bar{q}_{l}$. Finally, in addition to providing a powerful test of QCD, such measurements allow constraints to be placed on physics beyond the Standard Model. For example, a flavor-dependent anomalous quark chromomagnetic moment would modify [4] the emission rate of gluons for the different quark flavors, and would manifest itself in the form of an apparently flavor-dependent strong coupling.

The first such comparisons, of $\alpha_{s}$ for $b$ or $c$ with $\alpha_{s}$ for all flavors, were made at PETRA at a center-of-mass energy of $35 \mathrm{GeV}$ and were limited in precision to $\pm 0.41(c)$ and \pm 0.57 (b) [5] due to small data samples and limited heavy quark tagging capability. These studies made the simplifying assumptions that $\alpha_{s}$ is independent of flavor for all the non- $b$ and non- $c$ quarks, respectively. Measurements made using $Z^{0}$ decays recorded at LEP, under the first assumption, verified flavor-independence to a precision of \pm 0.012 on $\alpha_{s}^{b} / \alpha_{s}^{\text {all }}[6]$, and were insensitive to any difference among $\alpha_{s}$ values for the other flavors. The ALEPH Collaboration also measured $\alpha_{s}^{b c} / \alpha_{s}^{u d s}$ to a precision of \pm 0.023 , but in this case there is no sensitivity to a different $\alpha_{s}$ for $c$ and $b$ quarks. In addition, the OPAL Collaboration has measured $\alpha_{s}^{f} / \alpha_{s}^{\text {all }}$ for all five flavors $f$ with no assumption on the relative value of $\alpha_{s}$ for different flavors [7] and has verified flavor-independence to a precision of $\pm 0.026(b), \pm 0.09$ $(c), \pm 0.15(s), \pm 0.20(d)$ and $\pm 0.21(u)$. In that analysis the precision of the test was limited by the kinematic signatures used to tag $c$ and light quarks, which suffer from low efficiency and strong biases against events containing hard gluon radiation.

Here we present an improved test of the flavor-independence of strong interactions using a sample of approximately 150,000 hadronic $Z^{0}$ decay events produced by the SLAC Linear Collider (SLC) and recorded in the SLC Large Detector (SLD) [8] in data-taking runs between 1993 and 1995. The precise tracking capability of the Central Drift Chamber and the 120-million-pixel CCD-based Vertex Detector, combined with the stable, micron-sized beam interaction point (IP), allowed us to reconstruct topologically secondary vertices from heavy-hadron decays with high efficiency. High-purity samples of $Z^{0} \rightarrow b \bar{b}(g)$ and $Z^{0} \rightarrow c \bar{c}(g)$ events were then tagged on the basis of the reconstructed mass and momentum of the secondary vertex. Event containing no secondary vertex and no tracks significantly displaced from the IP were tagged as a high-purity $Z^{0} \rightarrow q_{l} \bar{q}_{l}(g)\left(q_{l}=u+d+s\right)$ event sample. The method makes no assumptions about the relative values of $\alpha_{s}^{b}, \alpha_{s}^{c}$, and $\alpha_{s}^{u d s}$. An important advantage of the method is that it has low bias against $\geq 3$-jet events. In addition to using an improved flavor-tagging technique, this analysis utilizes a data-sample size three times larger than that used in our previous measurement [9], based on the sample of roughly 50,000 $Z^{0}$ decay events collected in 1993, reached precisions of \pm 0.04 (uds), \pm 0.17 (c), and \pm 0.06 (b) on the basis of lifetime and decay multiplicity differences among hadrons containing light, $c$, and $b$ quarks. These features allow us to test the flavor independence of strong interactions to a precision higher by roughly a factor of three.

The organization of this thesis is as follows; chapter 2 reviews the perturbative QCD 
predictions and studies of the strong interaction in $e^{+} e^{-}$annihilation. Chapter 3 is devoted to a brief review of the SLC and the SLD. The hadronic event selection and flavor tagging are described in chapter 4 . The measurement of $R_{3}$ ratios and translation to the $\alpha_{s}$ ratios, using a next-to-leading-order calculation and taking into account heavy quark mass effects, are presented in chapter 5 . Chapter 6 summarizes the results and concludes this thesis.

Chapter 2

\section{The Strong Interaction in $e^{+} e^{-}$ Annihilation}

\subsection{Quarks and Interactions}

The quark parton model (QPM) was developed by Gell-Mann and Zweig [10] to explain the rich spectroscopy of mesons $(q \bar{q})$ and baryons $(q q q$ or $\bar{q} \bar{q} \bar{q})$ in terms of bound states of quarks $(q)$. If the quarks are assigned spin $1 / 2$, possess the quantum number flavor ( $f=u, d, s, c, b, t)$, which represents eigenstates of the weak interaction, electromagnetic charge $(+2 / 3$ for $u, c, t,-1 / 3$ for $d, s, b)$ and are triplets in terms of the color quantum number $(c=r, g, b)$ then the quantum numbers of all known hadrons can be created ${ }^{1}$. The color quantum number was introduced by Han and Nambu [11] to explain the existence of particles such as $\Delta^{++}$and $\Omega^{-}$which is symmetric quark state of $(u \uparrow u \uparrow u \uparrow)$ and $(s \uparrow s \uparrow s \uparrow)$, respectively, are forbidden by the Pauli exclusion principle.

The first direct evidence for quarks came from the observation at the SLAC-MIT experiment using deep inelastic electron-nucleon scattering [12], which revealed the existence of the point-like particle interaction from the properties of the Bjorken scaling structure functions [13].

In $e^{+} e^{-}$annihilation, the existence of quarks was demonstrated by the observation of

${ }^{1}$ There are a few hadron resonances that are difficult to interpret in terms of the Quark Parton Model (QPM) and may be candidates for so-called glueball states, particles which are bound states of gluons only. 
jets, the stream of final state particles coming from the production of a back-to-back quark and antiquark, in the Mark I experiment at SPEAR in 1975 [14]. Furthermore, the angular distribution of the jets confirmed the spin- $1 / 2$ of quarks and antiquarks [15]. Events containing three jets of hadrons were first observed at PETRA [16] in 1979 at center-of-mass energies around $20 \mathrm{GeV}$. Such events were interpreted in terms of the fundamental process $e^{+} e^{-} \rightarrow q \bar{q} g$, providing direct evidence for the existence of the gluon $(g)$ and its coupling to quarks.

Table 2.1: Fundamental spin- $\frac{1}{2}$ fermions. These masses are from [17].

\begin{tabular}{|c|c|c|c|c|}
\hline generation & flavor & symbol & charge & mass $\left(\mathrm{GeV} / c^{2}\right)$ \\
\hline \multicolumn{5}{|c|}{ quarks } \\
\hline first & up & $u$ & $2 / 3$ & $0.002 \sim 0.008$ \\
& down & $d$ & $-1 / 3$ & $0.005 \sim 0.015$ \\
\hline \multirow{2}{*}{ second } & charm & $c$ & $2 / 3$ & $1.0 \sim 1.6$ \\
& strange & $s$ & $-1 / 3$ & $0.1 \sim 0.3$ \\
\hline third & top & $t$ & $2 / 3$ & $180 \pm 12$ \\
& bottom & $b$ & $-1 / 3$ & $4.1 \sim 4.5$ \\
\hline \multicolumn{5}{|c|}{ leptons } \\
\hline first & e-neutrino & $\nu_{e}$ & 0 & $<1.5 \times 10^{-8}$ \\
& electron & $e$ & -1 & 0.0051 \\
\hline second & $\mu$-neutrino & $\nu_{\mu}$ & 0 & $<1.7 \times 10^{-4}$ \\
& muon & $\mu$ & -1 & 0.106 \\
\hline third & $\tau$-neutrino & $\nu_{\tau}$ & 0 & $<0.024$ \\
& tau & $\tau$ & -1 & $1.7770 \pm 0.0003$ \\
\hline
\end{tabular}

Table 2.2: Fundamental gauge bosons.

\begin{tabular}{|cccccc|}
\hline Force & gauge boson & symbol & charge & spin & mass $\left(\mathrm{GeV} / c^{2}\right)$ \\
\hline Strong & gluon & $g$ & 0 & 1 & 0 \\
\hline Electromagnetic & photon & $\gamma$ & 0 & 1 & 0 \\
\hline \multirow{2}{*}{ Weak } & W-boson & $W^{ \pm}$ & \pm 1 & 1 & $80.36 \pm 0.12[17]$ \\
& Z-boson & $Z^{0}$ & 0 & 1 & $91.187 \pm 0.007[17]$ \\
\hline Gravitational & graviton & $G$ & 0 & 2 & 0 \\
\hline
\end{tabular}

There are several different sorts of quarks $(u, d, c, s, t, b)$, these are assigned the flavor quantum numbers, which accounts for the variety of hadrons. The quark flavors are listed in
Table 2.1, as well as the leptons, which do not interact via the strong force. The interactions between these fermions are described by gauge theories; the transmitting particles are gauge bosons. Table 2.2 summarizes the fundamental forces and some properties of their gauge bosons. Flavors are only changed by charged weak interactions.

So far, a number of experiments support the $S U(2)_{L} \times U(1)$ electroweak theory, the Standard Model of electroweak interaction, developed by Glashow, Weinberg, and Salam [18], which unifies the electromagnetic and weak interactions. In this theory the left-handed quarks and leptons are arranged into isospin doublets, while the right-handed quarks and leptons are arranged in isospin singlet states. The Standard Electroweak Model is an effective theory and has three parameters ${ }^{2}$ which have to be determined from experiments. A set of useful parameterization is:

- The fine structure constant $\alpha=1 / 137.036$ [20], determined from the quantum Hall effect, is the electromagnetic coupling strength at $0 \mathrm{GeV}$ energy scale. Due to the vacuum polarization the coupling is modified, $1 / \alpha\left(M_{Z}\right)=127.9 \pm 0.1[21]$ at the energy scale of $M_{z}$.

- The Fermi coupling constant $G_{F}=1.16639 \times 10^{-5}$, determined from the lifetime of the muon [22].

- The Weinberg angle $\sin ^{2} \theta_{w}^{\text {eff }}=0.2315 \pm 0.0004$ [20], which is the electroweak mixing angle, directly measured by SLD [23] or from a combination of measurements for the $Z^{0}$ mass and other $Z^{0}$-pole observables, the $W$ mass, and neutral-current processes.

\subsection{Quantum Chromodynamics (QCD)}

Quantum Chromodynamics (QCD) is the $S U(3)$ gauge field theory which describes the strong interactions between quarks and gluons (color gauge boson). The Lagrangian describing the interactions of quarks and gluons is

$$
L_{Q C D}=\sum_{j}^{n_{f}}\left[\bar{q}_{j}\left(i \gamma^{\mu} \partial_{\mu}-m_{j}\right) q_{j}-g_{s}^{j}\left(\bar{q}_{j} \gamma^{\mu} T_{a} q_{j}\right) B_{\mu}^{a}-\frac{1}{4} G_{\mu \nu}^{a} G_{a}^{\mu \nu}\right]
$$

${ }^{2}$ not counting the masses of the fermions, Higgs boson, and CKM parameters [19] 
where $\alpha_{s}^{j}=g_{s}^{j^{2}} / 4 \pi$ is the strong coupling constant, $j=u, d, c, s, t, b$ is the quark flavor, and the $q_{j}$ are the 4-component Dirac spinors associated with each quark field, the $m_{j}$ are the quark masses, the $B_{\mu}^{a}$ are the Yang-Mills [24] gluon fields. The kinetic energy term is given by

$$
G_{\mu \nu}^{a}=\partial_{\mu} B_{\nu}^{a}-\partial_{\nu} B_{\mu}^{a}-g_{s}^{j} f_{a b c} B_{\mu}^{b} B_{\nu}^{c}
$$

The $f_{a b c}$ and $T_{a}(a=1, . .8)$ are the structure constants and the generators of the $\mathrm{SU}(3)$ algebra, which obey the commutation relations:

$$
\left[T_{a}, T_{b}\right]=i f_{a b c} T_{c}
$$

The requirement of invariance under local gauge transformation leads to a unique Lagrangian which severely restricts the possible interaction terms between quarks and gluons. This local gauge invariance is also necessary to ensure that the theory is renormalizable [25], by which the divergences in calculations are absorbed into finite numbers of physical values [26]. The last term in Eqn. 2.2 required by local gauge invariance implies that gluon themselves carry the color charge, and hence the existence of self-couplings (Fig. 2.1(b-c)). This is due to a non-Abelian nature of the $S U(3)$ gauge group.

Following Ref. [27], the essential features of QCD may be summarized as:

(i) quarks with spin $1 / 2$ exist as color triplets.

(ii) gluons with spin 1 as color octets.

(iii) the coupling $q \bar{q} g$ exists (Fig. 2.1(a)).

(iv) the coupling $g g g$ and $g g g g$ exists (Fig. 2.1(b-c)).

(v) the coupling constants in (iii) and (iv) are equal.

(vi) the coupling decreases as $1 / \ln Q^{2}$ with increasing energy scale $Q$.

Some properties of the strong coupling, (v) and (vi), are discussed in the following sections.
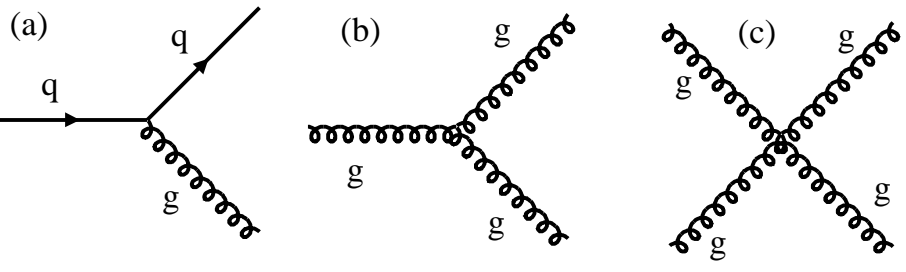

Figure 2.1: (a) quark-gluon, (b) triple-gluon, (c) four-gluon coupling.

\subsubsection{Strong Coupling and Perturbative QCD}

A characteristic feature of non-Abelian gauge theories is that the coupling strength $\alpha_{s}$ decreases with the four-momentum-transfer-squared $Q^{2}$ :

$$
\alpha_{s}\left(Q^{2}\right) \sim \frac{4 \pi}{\beta_{0} \log \left(Q^{2} / \Lambda^{2}\right)}
$$

where $\beta_{0}=11-2 n_{f} / 3, n_{f}$ is the number of active quark. Figure 2.2 summarizes the evidence of running $\alpha_{s}$ [28]. This means that for QCD, perturbation theory should work well at high $Q^{2}$ (short distance) where the quarks and gluons are quasi-free (asymptotic freedom), but breaks down at small $Q^{2}$ (large distance) where $\alpha_{s}\left(Q^{2}\right)$ becomes large and presumably confines quarks within hadrons.

The definition of the energy scale $\Lambda$ depends on the renormalization scheme, and is a matter of convention. The most commonly used prescription is the modified minimal subtraction $(\overline{M S})$ scheme [29], in which only the ultraviolet pole terms, and the constant terms arising from dimensional regularization ${ }^{3}[30]$ are absorbed into the coupling constant. Eqn. 2.4 can be written at $\mathcal{O}\left(\alpha_{s}^{2}\right)$ :

$$
\alpha_{s}\left(Q^{2}\right)=\frac{12 \pi}{\left(33-2 n_{f}\right) \ln \left(Q^{2} / \Lambda_{\overline{M S}}^{2}\right)+6\left(153-19 n_{f}\right) /\left(33-2 n_{f}\right) \ln \left(\ln \left(Q^{2} / \Lambda_{\overline{M S}}^{2}\right)\right)}(2.5)
$$

in the $\overline{M S}$ scheme.

As an example, a derivation of $\alpha_{s}$ value from a fit of $\mathcal{O}\left(\alpha_{s}^{2}\right)$ perturbative QCD to the observables in $e^{+} e^{-}$annihilation is shown in Fig. 2.3(a) [31]. The determined $\alpha_{s}$ values are

${ }^{3}$ The ultraviolet divergences in Feynman diagrams comes from the integration of internal momenta in 4 -dimensional space, which can be made finite by lowering the dimensions of the space-time to $N$, and then take the limit of the results as $N \rightarrow 4$. 
not internally consistent with one another within experimental errors. The reason for the scattering most likely comes from a truncation of the QCD perturbation series at finite order, which causes a residual dependence on the (scheme-dependent) renormalization scale $\mu$. This parameter is unphysical and should not enter at all into an exact infinite-order calculation, and its value is arbitrary. The SLD estimate of the renormalization scale uncertainty for each observable is shown in Fig. 2.3(b). It is apparent that the scale uncertainty is much larger than the experimental error.

The measured $\alpha_{s}$ values are conventionally extrapolated to $Q=M_{Z}$. Fig. 2.4 summarizes the current measurements of $\alpha_{s}$ along with the averaged value of $\alpha_{s}^{\text {all }}=0.118 \pm 0.005$ [32].

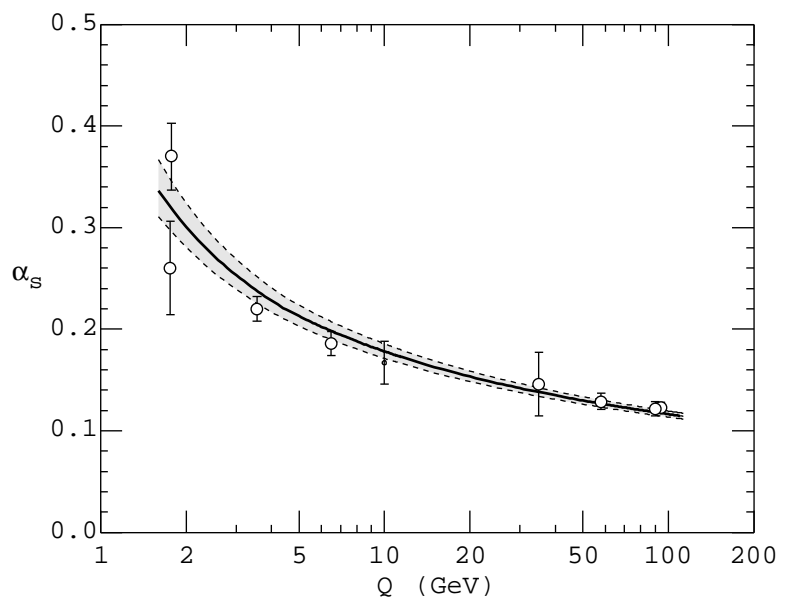

Figure 2.2: The values of $\alpha_{s}(Q)$ at the values of $Q$ where they are measured. The lines show the central values and the $\pm 1 \sigma$ limits of the world average. The figure clearly shows the decrease in $\alpha_{s}(Q)$ with increasing $Q$ [28].
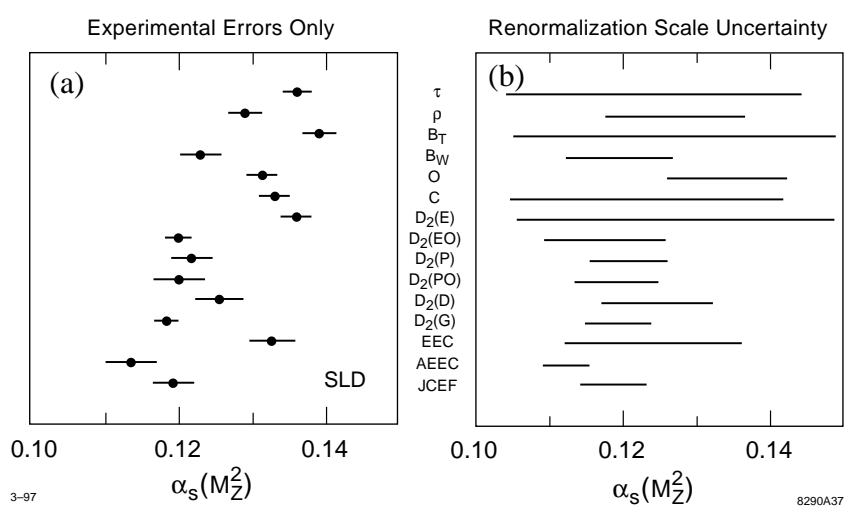

Figure 2.3: Determination of absolute value of $\alpha_{s}$ [31] using $\tau$; 1-Thrust [33], $\rho$; Heavy jet mass [34], $B_{t}, B_{w}$; Jet broadening (total, wide) [35], $O$; Oblateness [36], $C$; C-parameter [37], $D_{2}$; Differential two jet rates for E, E0, P, P0, Durham, Geneva algorithms, $E E C$; Energyenergy correlations [38], AEEC; Asymmetry of EEC, JCEF; Jet cone energy fraction [31].

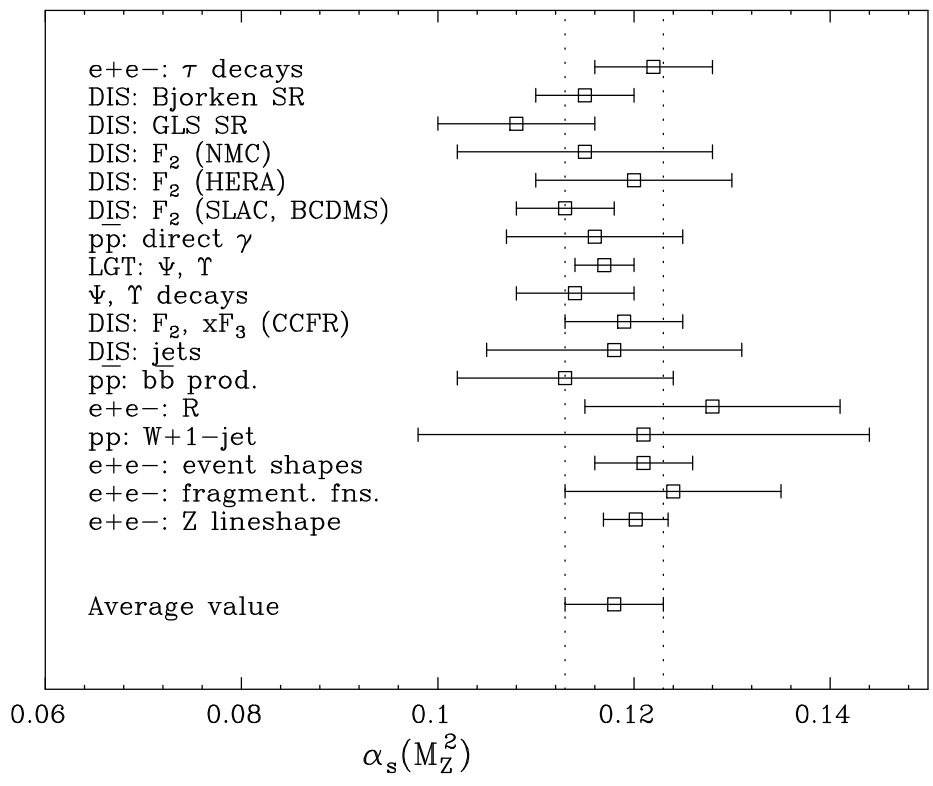

Figure 2.4: Summary of world $\alpha_{s}\left(M_{Z}^{2}\right)$ measurements [32]. The results are ordered vertically in terms of the hard scale $Q$ of the experiment. 


\subsubsection{Unitarity and Flavor Independence of QCD}

The existence of the gluon self-coupling vertices ( $g g g$ and $g g g g$ ) as well as the quark-gluonquark vertex $(q \bar{q} g)$, which is an another characteristic of non-Abelian gauge theory, and the requirement of unitarity of $\mathrm{QCD}$ demand the flavor independence of strong interactions.

The bare $q \bar{q} g$ vertex in Fig. 2.5(a) is remormalized with the various higher order corrections (Fig. 2.5(b)-(d)):

$$
\begin{aligned}
-i g_{r} \gamma_{\mu} T_{a} & =\frac{-i g_{o} \gamma_{\mu} T_{a}}{Z_{2} \sqrt{Z}_{3}}\left[1+\left(Z_{1}^{-1}-1\right)-2\left(Z_{2}^{-1}-1\right)+\left(Z_{3}-1\right)\right] \\
& =\frac{Z_{2} \sqrt{Z_{3}}}{Z_{1}}\left(-i g_{0} \gamma_{\mu} T_{a}\right)
\end{aligned}
$$

where $T_{a}(a=1, . .8)$ are the generators of $S U(3), Z_{i}$ is the renormalization factor, $g_{0}$ is the bare coupling, and the $g_{r}=Z_{2} \sqrt{Z}_{3} g_{0} / Z_{1}$ is the renormalized coupling.

As well as the coupling of $q \bar{q} g$ :

$$
g_{r}(q \bar{q} g)=\frac{Z_{2}^{m} \sqrt{Z_{3}^{g}}}{Z_{1}^{m}} g_{0} .
$$

the coupling of $g g g$ is renormalized :

$$
g_{r}(g g g)=\frac{Z_{2}^{g} \sqrt{Z_{3}^{g}}}{Z_{1}^{g}} g_{0}
$$

where $Z_{1}^{m}$ and $Z_{1}^{g}$ are the renormalization factor for $q \bar{q} g$ vertex and $g g g$ vertex, $Z_{2}^{m}$ is that for the self-energy of the quark matter field $q_{f}$, and $Z_{2}^{g}=Z_{3}^{g}$ is that for the self-energy of the gluon gauge field $g$. These are schematically shown in Fig. 2.6. In order to conserve probability the S-matrix must be unitary, which requires the Ward-Takahashi identity ${ }^{4}$. A generalized Ward-Takahashi identity applied for $\mathrm{QCD}^{5}$ implies that [40]:

$$
\frac{Z_{1}^{g}}{Z_{3}^{g}}=\frac{Z_{1}^{m}}{Z_{2}^{m}}
$$

${ }^{4}$ For instance, the Ward identity for a scattering of $q \bar{q} \rightarrow q \bar{q}$ with the intermediate states being two gauge boson states is [39]:

$$
k_{1}^{\mu} M_{\mu \nu}^{a b}=-i S^{a b} k_{2 \nu}
$$

where $M_{\mu \nu}^{a b}$ and $S^{a b}$ are the $q \bar{q} \rightarrow A_{\mu}^{a} A_{\nu}^{b}$ and $q \bar{q} \rightarrow c^{a \dagger} c^{b}$ amplitudes, where $A_{\mu}^{a}$ and $c^{a}$ are the gauge and ghost fields, respectively, and $k_{1}$ and $k_{2}$ are the four momenta of intermediate state gauge boson.

${ }^{5}$ In QED, the Ward-Takahashi identity implies that the renormalization factor for the vertex correction $\left(Z_{1}\right)$ and for the matter field self-energy $\left(Z_{2}\right)$ are equal.

Eqn. 2.11 and the presence of the three gluon vertex demands that the renormalized coupling between gluons $\left(g_{r}(g g g)\right)$ must be the same as that between gluons and quarks $\left(g_{r}(q \bar{q} g)\right)$ :

$$
g_{r}(q \bar{q} g) \equiv \frac{Z_{2}^{m} \sqrt{Z_{3}^{g}}}{Z_{1}^{m}} g_{0}=g_{r}(g g g) \equiv \frac{Z_{3}^{g} \sqrt{Z_{3}^{g}}}{Z_{1}^{g}} g_{0}
$$

Thus, the renormalized strong "charge" or the magnitude of the strong coupling must be universal and same for all quark flavors (f1, f2 ...); $g_{r}\left(q_{f 1} \bar{q}_{f 1} g\right)=g_{r}(g g g)=g_{r}\left(q_{f 2} \bar{q}_{f 2} g\right)$. If there were any flavor dependence of strong interaction, it would break the unitarity of QCD or might imply the existence of possible new physics. 
(a)

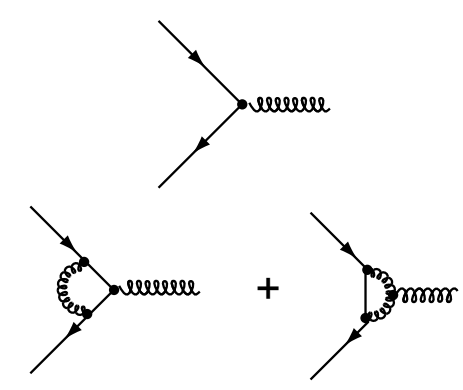

(c)
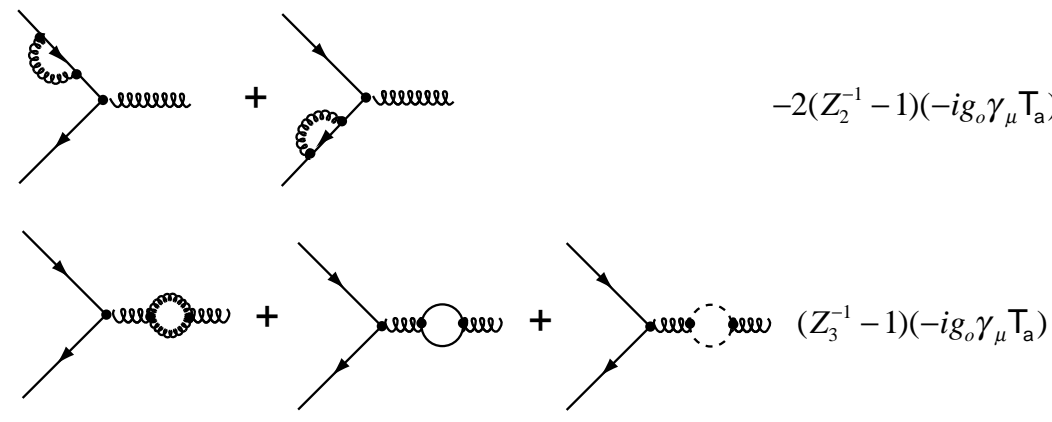

Figure 2.5: Feynman diagrams of (a) the bare $q \bar{q} g$ vertex; (b) the vertex corrections; (c) the quark self-energy corrections; (d) the corrections to the gluon propagator, and the corresponding vertex factors. The solid lines correspond to quarks, the helical curves represent gluons, and the dashed loop is the ghost contribution necessary for removing non-physical gluon polarization from the calculation at the chosen gauge [41] (a)

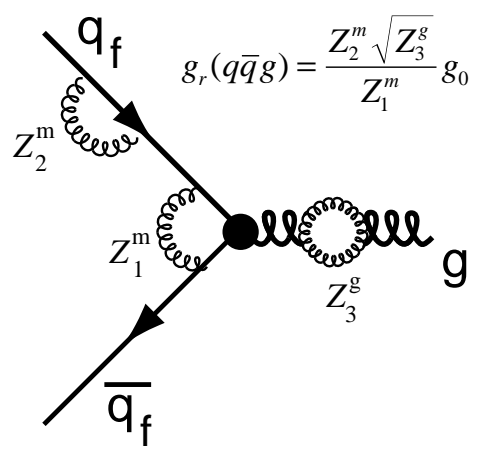

(b)

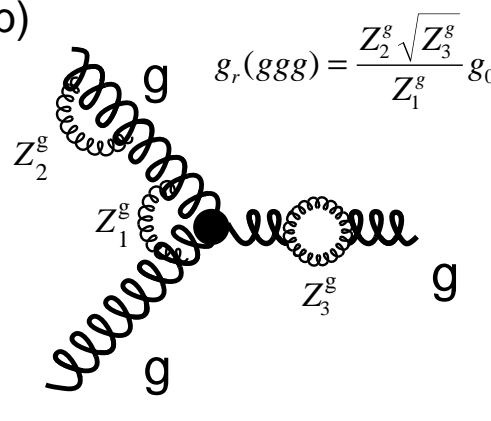

Figure 2.6: Schematic view of the renormalized couplings of $q \bar{q} g$ (a), and $g g g$ (b).

\subsection{Hadron Production in $e^{+} e^{-}$Collisions}

Characteristic of studies of the strong interaction in $e^{+} e^{-}$annihilation may be summarized as follows [42]. In $e^{+} e^{-}$annihilation, hadronic activity is, by construction, limited to the final state, making the study of hadronic events cleaner and simpler relative to lepton-hadron and hadron-hadron collisions, from both the experimental and theoretical points of view. On the experimental side, there are no remnants of the beam particles to add confusion to the interpretation of hadronic structures, and the hadronic center-of-mass frame coincides with the laboratory frame. On the theoretical side, the absence of hadrons in the incoming beams removes dependence on the limited knowledge of the parton density functions of hadrons, as well as rendering $\mathrm{QCD}$ calculations at a given order of perturbation theory easier to perform because there are generally fewer strong-interaction Feynman diagrams to consider. Electron-positron annihilation thus provides an ideal environment for precise test of QCD.

The process $e^{+} e^{-} \rightarrow$ hadrons is schematically shown in Fig. 2.7 [42], and can be classified in four phases:

(i) Production of a $q \bar{q}$ pair. This phase is described by the electroweak theory.

(ii) The primary quarks may radiate gluons, which may radiate $q \bar{q}$ or gluons, in consequence, leading to a parton shower. 
(iii) The colored objects produced in phase (ii) fragment into colorless hadrons.

(iv) The unstable hadrons produced in phase (iii) decay into the experimentally observed hadrons.

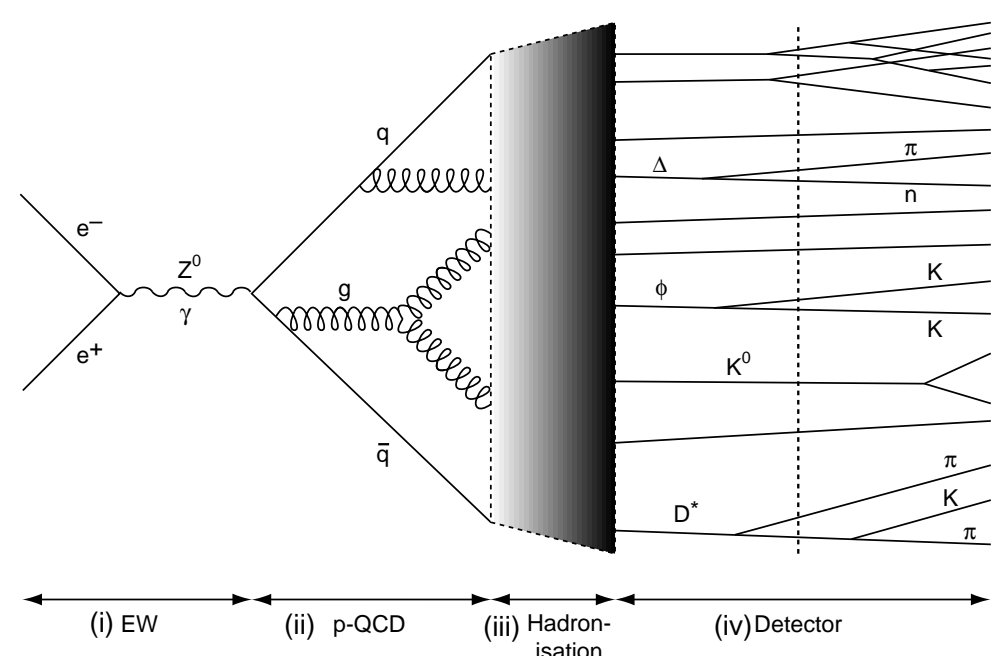

Figure 2.7: The phases in the process of hadronic production in $e^{+} e^{-}$annihilation [42]

The parton evolution in Phase (ii) is described by perturbative QCD. By contrast in large distance or low $Q^{2}$ interactions, the coupling strength increases so that quarks and gluons remain bound together or confined in color singlet states (Phase (iii)). Unfortunately, because the coupling strength is relatively large in 'soft' low $Q^{2}$ processes, the transition of partons into hadrons, known as hadronization or fragmentation, cannot be calculated in perturbative QCD. Non-perturbative calculations are very difficult; so-called 'lattice' QCD [43] calculations succeed in predicting some static hadronic properties such as masses and decay constants, but as yet there is little progress in the calculation of dynamical processes. At present, hadronization can only be described by phenomenological models, to be discussed later in this chapter.

The confinement of quarks and gluons within hadrons makes it difficult for us to compare experimental results with theoretical calculations. Although we can observe only hadronized

particles, we believe that we can peek at and go upstream to the primary quarks and gluons by investigating the final hadron distributions. According to perturbative QCD, a collection of final state hadrons called a jet would remember the property of the primary parton under the assumption of local parton-hadron duality [44].

In the following sections the above four phases will be described more in detail.

\subsection{1 $e^{+} e^{-}$annihilation into hadrons}

In the process of $e^{+} e^{-} \rightarrow f \bar{f}$ (phase (i) in Fig. 2.7), where $f$ denotes the final state fermion, a virtual gauge boson $\gamma / Z^{0}$ is exchanged. In the lowest-order approximation, the total cross section is given by [45]

$$
\sigma\left(e^{+} e^{-} \rightarrow f \bar{f}\right)=N_{c} \frac{4 \pi}{3} \frac{\alpha^{2}}{s}\left[q_{e}^{2} Q_{f}^{2}+2 q_{e} Q_{f} v_{e} v_{f} \operatorname{Re}(\chi)+\left(v_{e}^{2}+a_{e}^{2}\right)\left(v_{f}^{2}+a_{f}^{2}\right)|\chi|^{2}\right]
$$

where $\alpha$ is the electromagnetic fine structure constant, $Q_{f}$ is the electric charge of fermion $f$, $v_{f}$ and $a_{f}$ denote the vector and axial vector couplings of the $Z^{0}$ to fermion $f . N_{c}$ corresponds to the degrees of freedom of the color quantum number of fermion: 1 for leptons, 3 for quarks. The function $\chi$ describes the Breit-Wigner form of the $Z^{0}$ resonance:

$$
\chi=\frac{\alpha^{2}}{4 \sin ^{2} \theta_{w}} \frac{s}{\left(s-M_{Z}^{2}\right)+\Gamma_{Z}^{2} s^{2} / M_{Z}^{2}}
$$

where $M_{Z}=91.187 \mathrm{GeV} / c^{2}$ the $Z^{0}$ mass, $\Gamma_{Z}=2.49 \mathrm{GeV}$ the $Z^{0}$ width [20].

The total cross section for $e^{+} e^{-} \rightarrow$ hadrons as a function of the center-of-mass energy $(\sqrt{s})$ is shown in Fig. 2.8. At $\sqrt{s} \sim M_{z}$, the effect of the electromagnetic term and $\gamma-Z$ interference term (first term and second term in Eqn. 2.13) can be neglected and the $Z^{0}$ exchange term (third term in Eqn. 2.13) dominates by a factor of $\sim 800$. The cross section into hadronic final states is very large $(\sim 30 n b)$. Some properties of the $Z^{0}$ are shown in Table 2.4. 


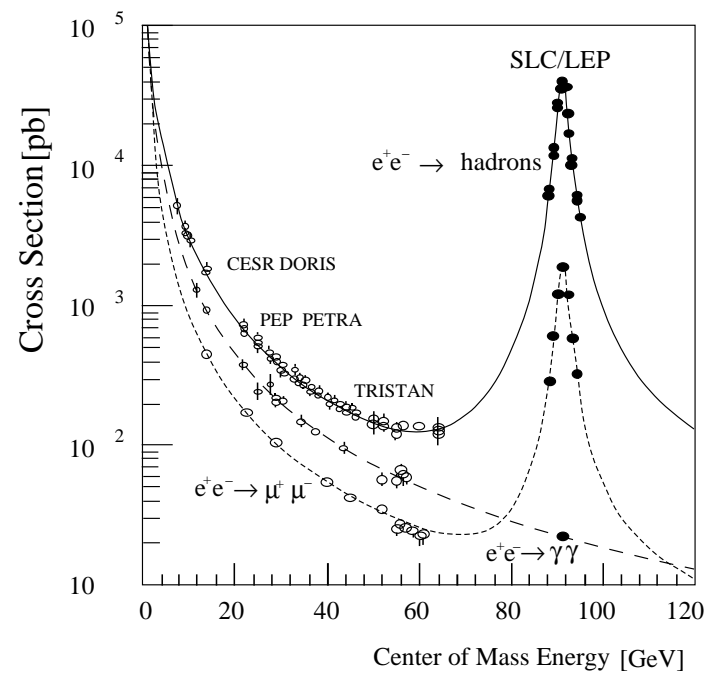

Figure 2.8: Total cross section for $e^{+} e^{-}$annihilation into hadrons and muon pairs as function of the center-of-mass energy. Also given is the two-photon cross section. Experimental data are compared with predictions from the Standard Model [46].

\begin{tabular}{|c|c|c|}
\hline fermion & $a_{f}$ & $v_{f}$ \\
\hline$\nu_{e}, \nu_{\mu}, \nu_{\tau}$ & $+\frac{1}{2}$ & $+\frac{1}{2}$ \\
$e^{-}, \mu^{-}, \tau^{-}$ & $-\frac{1}{2}$ & $-\frac{1}{2}+2 \sin ^{2} \theta_{w}$ \\
$u, c, t$ & $+\frac{1}{2}$ & $+\frac{1}{2}-\frac{4}{3} \sin ^{2} \theta_{w}$ \\
$d, s, b$ & $-\frac{1}{2}$ & $-\frac{1}{2}+\frac{2}{3} \sin ^{2} \theta_{w}$ \\
\hline
\end{tabular}

Table 2.3: The vector and axial vector couplings for fermions to the $Z^{0}$ gauge boson. $a_{f}=$ $T_{f}^{3}, v_{f}=T_{f}^{3}-2 \sin ^{2} \theta_{w} Q_{f}$, where $Q_{f}$ is the charge of the fermion, and $T_{f}^{3}$ is the third component of the weak isospin for the fermion.

\begin{tabular}{|c|c|}
\hline$M_{Z}$ & $91.187 \pm 0.007 \mathrm{GeV} / c^{2}$ \\
\hline$\Gamma_{Z}$ & $2.490 \pm 0.007 \mathrm{GeV}$ \\
\hline branching ratio (\%) & \\
\hline$e^{+} e^{-}$ & $3.366 \pm 0.008$ \\
$\mu^{+} \mu^{-}$ & $3.367 \pm 0.013$ \\
$\tau^{+} \tau^{-}$ & $3.360 \pm 0.015$ \\
$\nu \bar{\nu}$ & $20.01 \pm 0.16$ \\
$q \bar{q}^{-}$ & $69.90 \pm 0.15$ \\
\hline
\end{tabular}

Table 2.4: Some properties of the $Z^{0}$

\subsubsection{Parton Production}

In phase (ii) of Fig. 2.7, the primary quarks can radiate gluons and the subsequent gluons may radiate gluons or split into quarks. The parton structure can be calculated by perturbative QCD by using two approaches: matrix element (ME), the explicit calculation of Feynman diagrams for hard gluon emissions at the center of mass energy $(Q=\sqrt{s})$; and the parton shower (PS) method, employing explicit modeling of parton shower evolution based on the Altarelli Parisi equations [47].

\section{matrix element}

The cross section of the hadronic final state is derived by squaring the sum of the amplitudes, and may be expanded in terms of powers of $\alpha_{s}=g^{2} / 4 \pi$. Fig. 2.9a shows the zeroth order process $\left(\mathcal{O}\left(\alpha_{s}^{0}\right)\right)$ in which a $q \bar{q}$ pair is produced. Diagrams shown in Fig. $2.9 \mathrm{~b}$ and $2.9 \mathrm{c}$ contribute to the $q \bar{q}$ production in the power series of $\mathcal{O}\left(\alpha_{s}\right)$ and $\mathcal{O}\left(\alpha_{s}^{2}\right)$, respectively.

The differential cross section of $e^{+} e^{-} \rightarrow q \bar{q} g$ (Fig 2.10a) in $\mathcal{O}\left(\alpha_{s}\right)$ is given by

$$
\frac{d^{2} \sigma}{d x_{1} d x_{2}}=\sigma_{0} \frac{2}{3} \frac{\alpha_{s}}{2 \pi} \frac{x_{1}^{2}+x_{2}^{2}}{\left(1-x_{1}\right)\left(1-x_{2}\right)}
$$

where $\sigma_{0}$ is the lowest order Born cross section,

$$
\sigma_{0}=\frac{4 \pi \alpha^{2}}{3 s} N_{c} \sum_{f} Q_{f}^{2}
$$




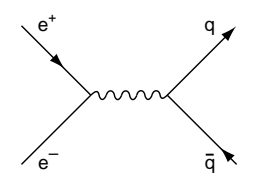

$\mathrm{O}\left(\mathrm{g}^{\circ}\right)$
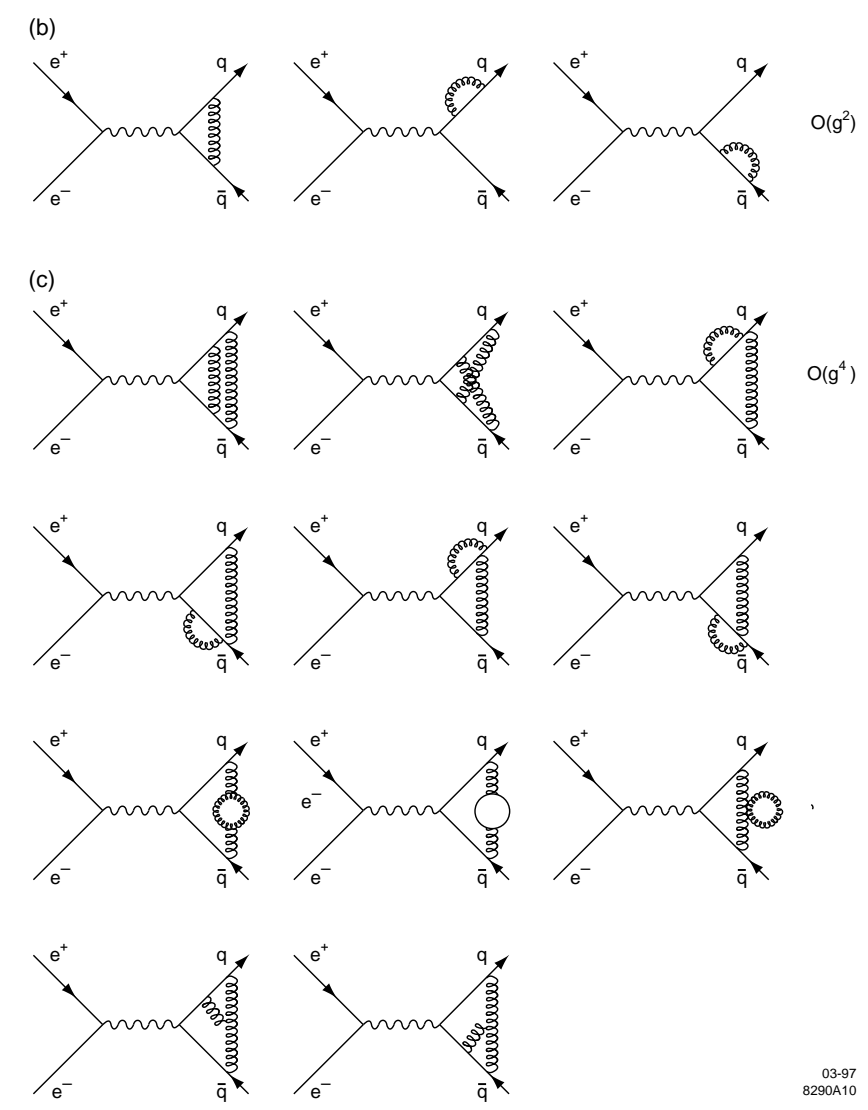

(a)
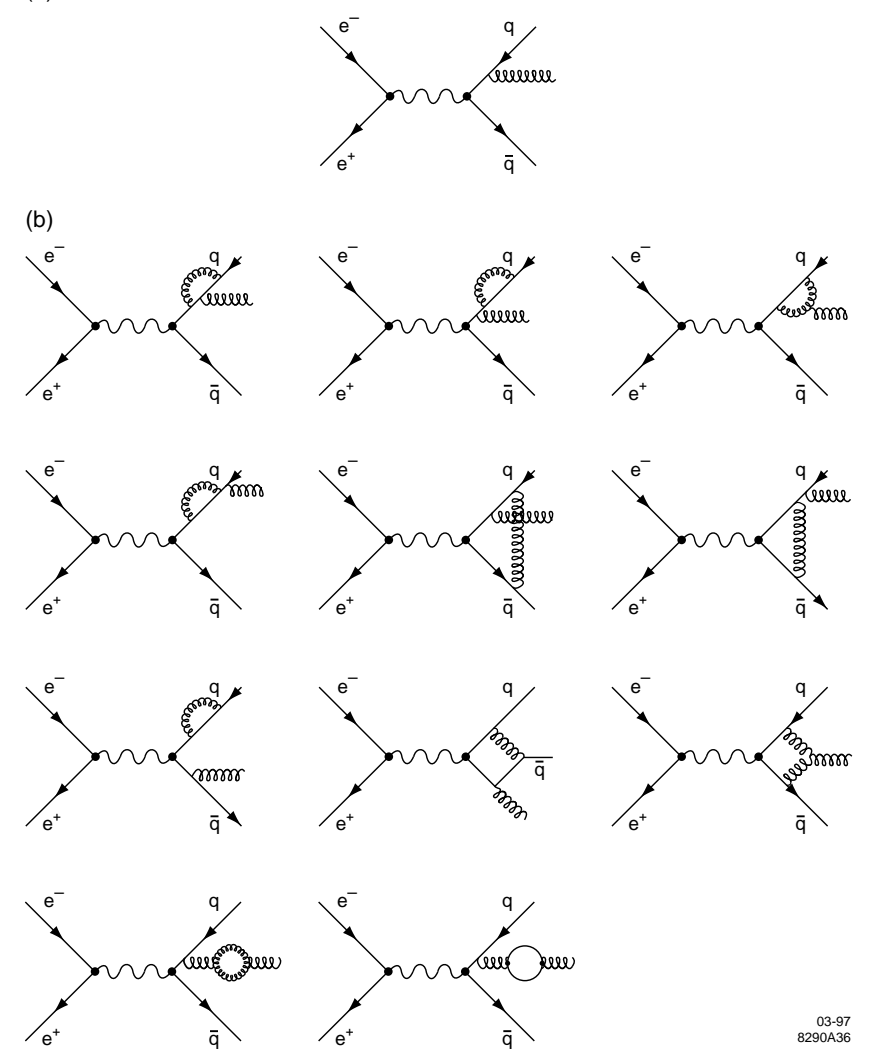

03997
8290936

Figure 2.10: Feynman diagrams for $e^{+} e^{-} \rightarrow q \bar{q} g$, (a) $\mathcal{O}\left(\alpha_{s}\right)$ and (b) $\mathcal{O}\left(\alpha_{s}^{2}\right)$ [42].

Figure 2.9: Feynman diagrams for $e^{+} e^{-} \rightarrow q \bar{q}$, (a) $\mathcal{O}\left(\alpha_{s}^{0}\right)$, (b) $\mathcal{O}\left(\alpha_{s}^{1}\right)$, and (c) $\mathcal{O}\left(\alpha_{s}^{2}\right)[42]$. 
$\mathrm{O}\left(\mathrm{g}^{2}\right)$
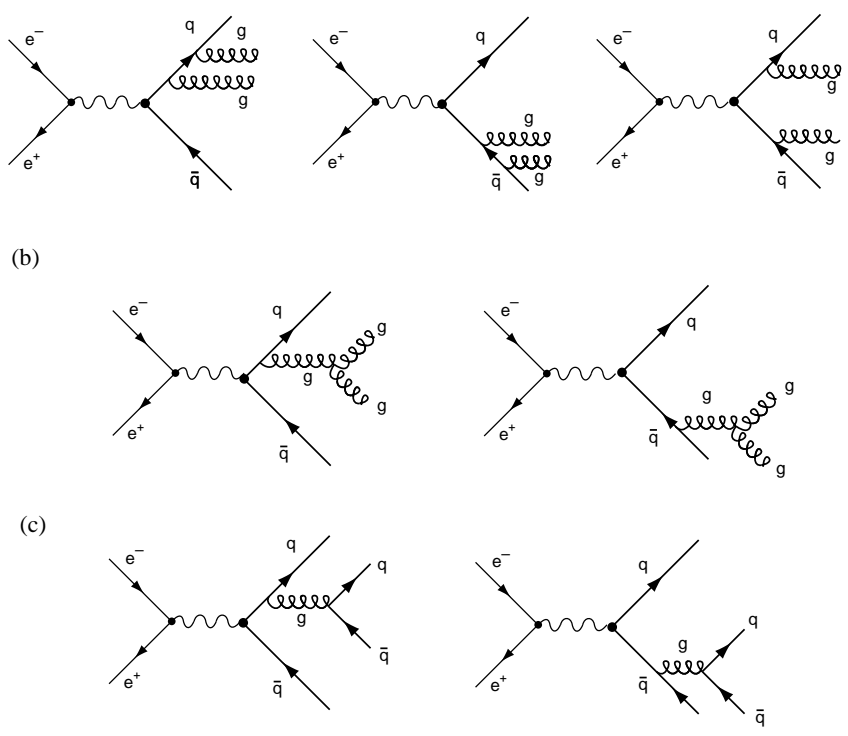

Figure 2.11: Feynman diagrams for four-jet events [42]. and $x_{i}=2 E_{i} / \sqrt{s}$ (particle energy normalized by center of mass energy), for $i=1: q, i=2$ : $\bar{q}, i=3: g$. Here, $N_{c}=3$ is the number of colors, $\alpha$ is the electromagnetic coupling, and $Q_{f}$ is the charge of quark flavor $f$.

This differential cross section diverges in the case that $x_{1} \rightarrow 1$ or $x_{2} \rightarrow 1$. The case $x_{1}=1$ and $x_{2}=1$ corresponds to the gluon emission with zero energy. This type of divergence is called an infrared divergence. The case $x_{1}=1$ but $x_{2} \neq 1$ corresponds to the situation when one of the quarks emits a gluon parallel to it. This is known as a collinear singularity.

Calculations including $\mathcal{O}\left(\alpha_{s}^{2}\right)$ contribution are very involved; the amplitudes corresponding to all the diagrams in Fig. 2.9-2.10 must be summed and squared, including the gluon self-coupling diagrams (Fig 2.11b). $\mathcal{O}\left(\alpha_{s}^{2}\right)$ calculations have been performed for observables such as 3-jet rate:

$$
R_{3}=\frac{\sigma_{3-j e t}}{\sigma_{t o t}}
$$

where $\sigma_{t o t}$ and $\sigma_{3-j e t}$ is the total hadronic- and 3-jet event cross section, respectively.

$\alpha_{s}$ can be measured from the number of 3-jet events, because the cross section for hard gluon radiation depends on it (Eq. 2.15). The collinear and infra-red divergences are handled by dimensional regularization and by isolating the singular regions of phase space using a parton resolution criteria such as scaled invariant mass $y_{i j}=m_{i j}^{2} / s$. For each pair of partons $i, j$, one requires that all $y_{i j}>y_{\text {cut }}$ for the event to be counted as a 3-jet event, otherwise it is counted as a 2-jet event. Note, however, that jet cross sections now depend on $y_{c u t}$ The perturbative QCD prediction for the differential cross section in $y_{\text {cut }}$ up to $\mathcal{O}\left(\alpha_{s}^{2}\right)[48,49]$ is

$$
\frac{1}{\sigma_{\text {tot }}} \frac{d \sigma\left(y_{\text {cut }}\right)}{d y_{\text {cut }}}=A\left(y_{\text {cut }}\right)\left(\frac{\alpha_{s}}{2 \pi}\right)+\left[B\left(y_{\text {cut }}\right)+A\left(y_{\text {cut }}\right) 2 \pi b_{0} \operatorname{lnf}\right]\left(\frac{\alpha_{s}}{2 \pi}\right)^{2}
$$

where $b_{0}=\left(33-2 n_{f}\right) / 12 \pi$; and $n_{f}=5$ at $\sqrt{s}=M_{Z}$ is the number of active quark flavors; $f=\mu / s$ is the renormalization scale factor.

Calculations beyond $\mathcal{O}\left(\alpha_{s}^{2}\right)$ are very few ${ }^{6}$. A few observables can be calculated by resummation technique ${ }^{7}[52]$ to access the leading terms beyond $\mathcal{O}\left(\alpha_{s}^{2}\right)$ recently developed just for massless parton.

${ }^{6}$ For instance in $e^{+} e^{-}$annihilation, $\mathcal{O}\left(\alpha_{s}^{3}\right)$ calculations: the ratio of hadronic to $\mu$ pair production; $R=\sigma\left(e^{+} e^{-} \rightarrow\right.$ hadrons $) / \sigma\left(e^{+} e^{-} \rightarrow \mu^{+} \mu^{-}\right)$for massless quarks [50], and the ratio of hadronic $\left(B_{h}\right)$ to leptonic $\left(B_{l}\right) \tau$-lepton decay branching ratios; $R_{\tau}=B_{h} / B_{l}[51]$ are available.

${ }^{7} \mathrm{~A}$ resummation technique utilizes the leading and next-to-leading logarithmic terms which can be calcu- 


\section{Parton Shower}

When it comes to multi-parton structures, the calculation for the cross section in the ME approach becomes unrealistic due to the tremendous numbers of Feynman diagrams contributing. One alternative is the parton shower approach based on the framework of the leading logarithm approximation (LLA) [47, 53], where the leading term in the perturbative QCD expansion is retained.

In the shower formulation, the kinematics of each $a \rightarrow b c$ branch is given in terms of two variables, $Q^{2}$ and $z$. $Q^{2}$ has dimensions of squared mass, and is related to the mass or transverse momentum scale of the branching. Variable $z$ gives the sharing of energy and momentum between the two daughters. $b$ takes a fraction $z$ and $c$ takes a fraction $1-z$.

The probability for a parton to branches is given by the Altarelli Parisi evolution equation [47] :

$$
\begin{aligned}
d P_{a} & =\sum_{b, c} \frac{\alpha_{s}}{2 \pi} P_{a \rightarrow b c}(z) d z d t \\
P_{q \rightarrow q g}(z) & =C_{F} \frac{1+z^{2}}{1-z} \\
P_{g \rightarrow g g}(z) & =N_{C} \frac{(1-z(1-z))^{2}}{z(1-z)} \\
P_{g \rightarrow q \bar{q}}(z) & =T_{R}\left(z^{2}+(1-z)^{2}\right)
\end{aligned}
$$

where $C_{F}=4 / 3, N_{C}=3, T_{R}=n_{f} / 2$, and $t=\ln \left(Q^{2} / \Lambda_{P S}^{2}\right)$ is the evolution scale parameter. After a number of steps, these partons have virtualities $Q^{2}<\Lambda_{P S} \sim 1 \mathrm{GeV}$ and the shower is terminated.

The parton shower models based on these frameworks describe the coherent effects of soft gluons inside a jet and particle flow in between jets as well as hard gluon radiation in an approximate manner.

lated to all orders in $\alpha_{s}$. In order to make reliable predictions it is necessary to combine them with the $\mathcal{O}\left(\alpha_{s}^{2}\right)$ calculations taking into account overlapping terms. This procedure is expected a priori to yield formulae which are less dependent on the renormalization scale.

\subsubsection{Hadronization}

As described in sect. 2.2.1, the dependence of the momentum transfer, $Q^{2}$ on the strong coupling is $\alpha_{s} \sim 1 / \log \left(Q^{2} / \Lambda^{2}\right)$. We can see that as the parton shower evolves, $Q^{2}$ decreases and the coupling becomes quite large. This implies that no free quarks are found in nature. They must be confined within color-singlet hadrons. The hadronization process, phase (ii) of Fig. 2.7, is intrinsically non-perturbative. Dynamics of the confinement of quarks into hadrons is not yet well-understood.

In the absence of a deterministic calculation, however, several phenomenological models have been developed and implemented in Monte Carlo event generators.

\section{String Fragmentation}

A QCD-inspired model of fragmentation is the string model [54]. The basic idea is that the confinement potential of QCD corresponds to a color flux tube (i.e. string) which is stretched between the partons in an overall singlet state. In this picture, a quark or anti quark corresponds to an end of the string, while gluons correspond to kinks on the string (Fig. 2.12).
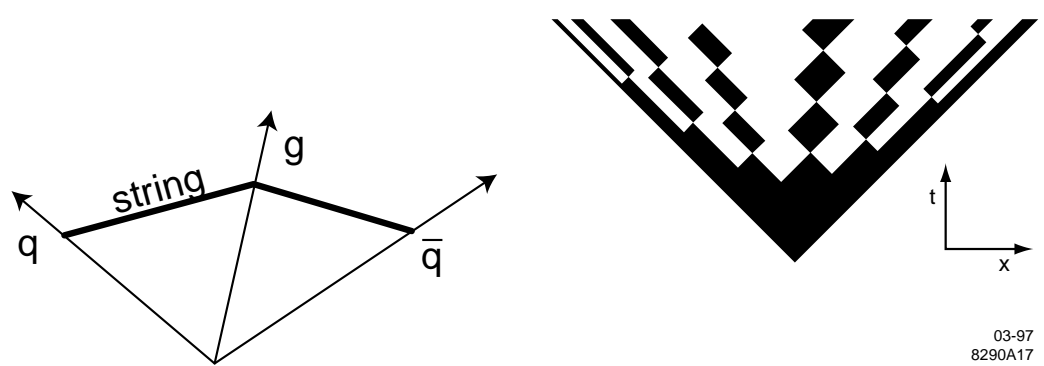

Figure 2.12: Schematic views of $q \bar{q} g$ string (left) and hadronization (right) [42] in string model.

The Lund string model [55] invokes the idea of quantum tunneling, which lead to string breakups. The equations of motion for the end-points of this relativistic string are

$$
\frac{d p}{d t}= \pm \kappa
$$


where $p$ is the momentum of the end-point quarks and the $+(-)$ sign refers to the left (right) end of the string. The probability for string breaking is

$$
\exp \left(-\frac{\pi m_{\perp}^{2}}{\kappa}\right)=\exp \left(-\frac{\pi m^{2}}{\kappa}\right) \exp \left(-\frac{\pi p_{\perp}^{2}}{\kappa}\right)
$$

where $m$ is the mass of the created quarks; this is like a tunneling probability through a potential barrier. JETSET7.4 [56], an implementation of Parton Shower calculation plus string fragmentation, reproduces data quite successfully and is used as a reference Monte Carlo generator by SLD.

\section{Cluster Fragmentation}

An alternative model is the cluster fragmentation model implemented in the HERWIG Monte Carlo generator [57]. This hadronization model is inspired by the preconfinement [58] characteristic of perturbative QCD. Preconfinement is the property of QCD whereby a parton almost always finds itself nearby in momentum space to a parton carrying opposite color charge, whenever the partons have evolved from high to low virtuality. It is assumed that such colorless objects called clusters therefore possess the properties of a hadron resonance. At the end of the parton shower evolution, gluons which posses two color indices are forcibly split into $q \bar{q}$ pairs, and pairs of adjacent partons are associated into colorless clusters. Hadrons are produced through two-body, isotropic decays of the clusters. The hadron species are selected in proportion to phase-space (hadron mass) and spin factors.

\section{Independent Fragmentation}

The independent fragmentation model was inspired by the Field-Feynman model [59]. An initial quark $q$ with energy $W$ becomes paired into a hadron $\left(q \bar{q}_{1}\right)$ carrying energy fraction $W z_{1}$. Remnant quark $q_{1}$ becomes paired into a hadron $\left(q_{1} \bar{q}_{2}\right)$ carrying energy fraction $W z_{2}\left(1-z_{1}\right)$ and so on. This model has been gradually phased out, since it does not describe the string effect, which is an experimentally well established phenomenon [60]; in $q \bar{q} g$ events fewer hadrons are produced in the region between the two quarks than between a quark and the gluon. 


\section{Fragmentation Functions}

The fragmentation function $f(z)$ expresses the probability distribution of $z=E_{h} / E_{q}$, the fraction of a primordial quark energy $\left(E_{q}\right)$ carried by a fragmented hadron $\left(E_{h}\right)$. If one requires the iterative procedure of the fragmentation to yield the same result irrespectively of whether one starts from the $q$ end or the $\bar{q}$ of the string in Fig. 2.12(a), only one function is allowed: the "Lund symmetric fragmentation function" [55],

$$
f(z) \propto \frac{(1-z)^{a}}{z} \exp \left(\frac{-b m_{\perp}^{2}}{z}\right) .
$$

where $m_{\perp}^{2}=p_{\perp}^{2}+m^{2}$ is the hadron transverse mass-squared w.r.t. the initial quark direction. Parameters $a$ and $b$ used in the SLD Monte Carlo are listed in Table. 3.1

If the fragmented parton is a heavy quark $Q$, it needs to lose only a small fraction of its energy [61]. The resulting heavy-flavored hadron $H_{Q}$ will carry a large fraction of the original energy: $z=E_{H} / E_{Q} \sim 1$. The Bowler function [62] is a function within the framework of the string model. Eqn. 2.25 is modified:

$$
f(z) \propto \frac{z^{a_{1}}}{z^{1+r_{Q} b m_{Q}^{2}}}\left(\frac{1-z}{z}\right)^{a_{2}} \exp \left(\frac{-b m_{\perp}^{2}}{z}\right) .
$$

$r_{Q}$ is the parameter so as to extrapolate smoothly between this form and the Lund symmetric one.

The Peterson function [63] is the best known one for heavy quark fragmentation that well agrees with data $[64,65,66]$. The energy denominator in the amplitude for this process is

$$
\begin{aligned}
\delta E & =E_{Q}-E_{H}-E_{q} \\
& \simeq \frac{m_{Q}^{2}}{2 p}-\frac{m_{H}^{2}}{2 p}-\frac{m_{q}^{2}}{2(1-z) p} \sim 1-\frac{1}{z}-\frac{m_{q}^{2} / m_{Q}^{2}}{1-z}
\end{aligned}
$$

where $p$ is the initial $Q$ momentum, and $m_{Q} \simeq m_{H}$ is assumed. All other factors are approximated by constants, apart from a longitudinal phase space factor $d^{3} p_{H} / E_{H} \simeq \pi d p_{T}^{2} d z / z$. This gives the Peterson fragmentation function [63]

$$
f(z) \propto \frac{1}{z\left(1-\frac{1}{z}-\frac{\epsilon_{Q}}{1-z}\right)^{2}}
$$

where $\epsilon_{Q}$ is a parameter expected to be proportional to $m_{Q}^{-2}$, listed in Table. 3.1.

\subsection{Current Experimental Status}

In this section we briefly discuss current experimental tests of flavor independence of strong interactions, as well as the possibility of anomalous moments which might modify the $q \bar{q} g$ vertex in the heavy quark sector.

\subsubsection{Measurements of $\alpha_{s}$ ratios}

\section{TASSO}

The TASSO Collaboration [5] at PETRA $e^{+} e^{-}$collider carried out the first test of the flavor independence of $\alpha_{s}$ at center of mass energy of $\sqrt{s}=35 \mathrm{GeV}$ using approximately 31,000 hadronic events. Events containing charm quarks were identified by exclusive reconstruction of $D^{*} \rightarrow \pi D^{0}$ meson decays and the Energy-energy correlation (EEC) [38] was used for the measurements of the strong coupling. They obtained $\alpha_{s}^{c} / \alpha_{s}^{\text {all }}=0.91 \pm 0.38$ (stat) \pm 0.15 (syst). This technique of selecting charm decays requires the charm meson to be carrying a large fraction of the energy available to it, which limits the acceptance for events with very hard gluons. Also, charm events containing the appropriate $D^{*} \rightarrow \pi D^{0}$ cascades only make up a small fraction $(7 \%)$ of the total and the exclusive decay modes used to reconstruct the $D^{0}$ have small branching fractions. These factors lead to an undesirably small efficiency for tagging charm events and hence to a large statistical error. Events containing $b$ quarks were enriched by weighting the decay vertices separated from the beam interaction region using the long $B$ hadron lifetime. 516 events were selected as a $b$-rich sample with a purity of $68 \%$. They obtained $\alpha_{s}^{b} / \alpha_{s}^{\text {all }}=1.17 \pm 0.50($ stat $) \pm 0.28$ (syst) from the EEC.

\section{Tests at the $Z^{0}$ Resonance}

The era of experimentation at the $Z^{0}$ pole with $e^{+} e^{-}$colliders has allowed test of the flavor independence of QCD with greater precision. A common method is to use identified leptons with large momentum and transverse momentum relative to the nearest jet axis as a tag of $b$ or $c$ quarks. Depending on the cuts placed on the lepton momenta, this method could also bias the event sample away from those events containing hard gluon radiation. Another method is lifetime tagging using long lifetime of heavy hadrons (see Sect. 5.6.2.). Jet clus- 
tering algorithms are commonly used to define the number of jets in an event, and will be discussed in Sect. 5.2.1.

L3

The L3 collaboration [67] found $\alpha_{s}^{b} / \alpha_{s}^{u d s c}=1.00 \pm 0.05$ (stat) \pm 0.06 (syst) from the measured 3jet rate. From 110,000 hadronic events approximately $2900 b$ events were selected identifying the muons or electrons coming from the semileptonic decay of the $b$ quark. The $b$ purity was evaluated to be $87 \%$. The jet rates in the selected samples of hadronic and inclusive lepton samples were determined using the JADE algorithm [68] at $y_{\text {cut }}=0.05$.

\section{DELPHI}

The DELPHI collaboration measured $\alpha_{s}^{b} / \alpha_{s}^{u d s c}$ using 356,000 hadronic events collected in 1990 and 1991 and that subset containing leptons with large momenta and transverse momenta. The purities of $b$-sample are $76 \%$ and $68 \%$ for muon and electron samples, respectively. They measured $\alpha_{s}^{b}=0.118 \pm 0.004($ stat $) \pm 0.003($ syst $) \pm 0.008$ (scale) by fitting the momentum and transverse momentum spectra of the lepton candidates in both two- and three-jet event samples simultaneously using the spectra predicted from the Monte Carlo simulation for $b, c$, and background events [69]. A comparison with $\alpha_{s}$ for all flavors yielded the relative strength $\alpha_{s}^{b} / \alpha_{s}^{u d s c}=1.00 \pm 0.04$ (stat) \pm 0.03 (syst) from jet rates using the JADE algorithm with the E0 scheme at $y_{c u t}=0.06$. the P-scheme, the Durham and the Geneva algorithms [70] were also used as cross checks.

Recently they updated their measurement using 2.8 million hadronic $Z$ decays collected during 1992-1994 [71]. $b$ and $u d s$ event flavor was identified using impact parameter tag. The purity and efficiency for $b$ tag sample were $85 \%$ and $55 \%$, respectively. Those for $u d s$ tag sample were approximately $80 \%$ and $80 \%$, respectively. Using the Durham algorithm at $y_{c u t}=0.02$ they found: $\alpha_{s}^{b} / \alpha_{s}^{u d s}=1.007 \pm 0.005$ (stat) \pm 0.007 (frag.) \pm 0.005 (theo.) where frag. and theo. denote the uncertainty from fragmentation model and theoretical uncertainty from renormalization and $b$ mass ambiguity, respectively. They also derived the $b$ quark mass at the $M_{Z}$ scale defined in the $\overline{M S}$ renormalization scheme:

$$
m_{b}\left(M_{z}\right)=2.67 \pm 0.25(\text { stat. }) \pm 0.34(\text { frag. }) \pm 0.27(\text { theo. }) \mathrm{GeV} / c^{2}
$$

assuming the flavor independence of strong interaction.

\section{ALEPH}

The ALEPH collaboration [72] measured the ratios $\alpha_{s}^{b} / \alpha_{s}^{u d s c}=1.002 \pm 0.009$ (stat.) \pm 0.005 (syst.) \pm 0.021 (theo.) and $\alpha_{s}^{u d s} / \alpha_{s}^{c b}=0.971 \pm 0.009$ (stat) \pm 0.011 (syst) \pm 0.018 (theo.) using about 950,000 hadronic events collected during 1991 and 1992. The analysis was based on eventshape variables (Thrust [33], C-parameter [37]), JADE [68] and Durham [70] jet clusterings. Two $b$-quark samples were enriched by lepton tagging and lifetime tagging, and a light-quark sample was enriched by lifetime anti-tagging; the flavor purities were $88 \pm 2 \%, 86 \pm 3 \%$, $81 \pm 3 \%$, respectively

\section{OPAL}

The OPAL collaboration performed the first truly comprehensive study of the flavor independence of the strong interaction. They used high $p_{t}$ leptons to form a $b$ tag, exclusively reconstructed $D$ mesons for the charm tag, fast $K_{s}^{0}$ for a strange tag $\left(X_{k}=2 E_{k} / \sqrt{s}>\right.$ 0.4 ; the scaled energy fraction to the primary quark), and fast pions, protons, and kaons $\left(0.7<X_{\pi, K, p}<1.07\right)$ as a tag of light $(u d s)$ flavors. The differential 2-jet rate was measured using JADE with E0 algorithm, and a grand unfolding was done to obtain the ratios of couplings $[7]$ :

$$
\begin{aligned}
& \left.\alpha_{s}^{b} / \alpha_{s}^{\text {all }}=1.021 \pm 0.013(\text { stat. }) \pm 0.023 \text { (syst. }\right) \\
& \left.\left.\alpha_{s}^{c} / \alpha_{s}^{\text {all }}=0.912 \pm 0.067 \text { (stat. }\right) \pm 0.061 \text { (syst. }\right) \\
& \alpha_{s}^{s} / \alpha_{s}^{\text {all }}=1.141 \pm 0.043(\text { stat. }) \pm 0.142(\text { syst. }) \\
& \left.\left.\alpha_{s}^{d} / \alpha_{s}^{\text {all }}=0.933 \pm 0.087 \text { (stat. }\right) \pm 0.175 \text { (syst. }\right) \\
& \left.\left.\alpha_{s}^{u} / \alpha_{s}^{\text {all }}=0.951 \pm 0.103 \text { (stat. }\right) \pm 0.182 \text { (syst. }\right)
\end{aligned}
$$

The large systematic errors on the light quark coupling ratios are due to the uncertainties in basing a tag on the identity of the fastest particle in an event, as this is uncharted territory for many of the Monte Carlo models of hadron production. The large statistical errors on these quantities are a consequence of the inefficiency of the light flavor tags. A 1995 paper [73] by OPAL has extended the repertoire of techniques used in the measurement 
of $\alpha_{s}$ for the flavor tagged samples. They used a detached vertex $b$ tag. Their result is $\alpha_{s}^{b} / \alpha_{s}^{\text {all }}=0.994 \pm 0.015 \pm 0.011$.

\section{SLD}

The SLD collaboration carried out an $\alpha_{s}$ ratio measurement using 50,000 hadronic event collected during the 1993 run [9]. In an attempt to obtain the best possible statistical precision for all flavors, we have chosen to pursue an "inclusive" analysis to test the flavor independence of the strong interaction. The flavor tag was accomplished by an impact parameter tag (see Sect. 5.6.2). The published result is:

$$
\begin{aligned}
\frac{\alpha_{s}^{\text {uds }}}{\alpha_{s}^{\text {all }}} & =0.987 \pm 0.027(\text { stat }) \pm 0.022(\text { syst }) \pm 0.022(\text { theor } y) \\
\frac{\alpha_{s}^{c}}{\alpha_{s}^{\text {all }}} & =1.012 \pm 0.104(\text { stat }) \pm 0.102(\text { syst }) \pm 0.096(\text { theor } y) \\
\frac{\alpha_{s}^{b}}{\alpha_{s}^{\text {all }}} & =1.026 \pm 0.041(\text { stat }) \pm 0.041(\text { syst }) \pm 0.030(\text { theor } y)
\end{aligned}
$$

The dominant experimental systematic errors arise from the uncertainties in the modeling of heavy hadron decays. The theoretical uncertainties are dominated by the variation in the results obtained with the six different jet algorithms (E, E0, P, P0, Durham and Geneva, see Sect. 5.2.1) used in the analysis, which are presumably caused by uncalculated higher-order terms in the predictions for jet production rates.

\section{Summary}

The measurements of $\alpha_{s}$ (flavors) $/ \alpha_{s}($ all $)$ at $Z^{0}$ are summarized in Fig. 2.14. In all but the OPAL analysis involving five flavors, assumptions need to be made about the relative strength of the strong coupling for the other quark flavors, as no sample of events is $100 \%$ pure, and the backgrounds must be subtracted. The most common approach is to assume that $\alpha_{s}^{u}=\alpha_{s}^{d}=\alpha_{s}^{s}=\alpha_{s}^{c}$ and then proceed with the background subtraction. We only make the weak assumption that $\alpha_{s}^{u}=\alpha_{s}^{d}=\alpha_{s}^{s}$.

\subsubsection{Anomalous Chromomagnetic Moment}

Finally, in addition to providing a powerful test of QCD, such measurements allow constraints to be placed on physics beyond the Standard Model. For example, a flavor-dependent anoma- lous quark chromomagnetic moment would modify the emission rate of gluons for different quark flavors, and would manifest itself in the form of an apparently flavor-dependent strong coupling.

In general one might expect new high-mass scale dynamics to couple with the massive third generation fermions. The possibility and influence of an anomalous chromomagnetic moment on the spectrum of gluon radiation associated with heavy quark production has recently been proposed [4]. In terms of an anomalous chromomagnetic moment $\kappa$, the QCD Lagrangian is modified:

$$
L=g_{s} \bar{q}_{j} T_{a}\left(\gamma^{\mu}+i \frac{\kappa}{2 m_{j}} \sigma_{\mu \nu} k^{\nu}\right) q_{j} G_{\mu}^{a}
$$

where $k$ is the outgoing gluon momentum from the $q_{j} \bar{q}_{j} g$ vertex. Anomalous non-zero $\kappa$ yields, in effect, a modified gluon radiation rate (at the leading order of $\alpha_{s}$ and $\kappa$ ):

$$
\frac{\sigma_{q_{j} \bar{q}_{j} g}}{\sigma_{q_{j} \bar{q}_{j}}} \simeq \frac{\alpha_{s}}{\pi} \frac{\kappa^{2} s}{18 m_{j}^{2}} \frac{v_{j}^{2}+1.25 a_{j}^{2}}{v_{j}^{2}+a_{j}^{2}}
$$

where $a_{j}$ and $v_{j}$ are the axial and vector coupling, respectively, of the quark flavor $j$ in the weak interaction. If $\kappa$ were non-zero for a given quark flavor, the measured value of $\alpha_{s}$ for that flavor would be distorted.

The gluon energy distribution at NLC $(t \bar{t} g)$ will yield greatly increased sensitivity to nonzero $\kappa$, however, an experimental constraint was obtained from the gluon energy spectrum in $b \bar{b} g$ events [74]:

$$
-0.106<\kappa<0.044
$$

with $95 \%$ confidence level limit for $b \bar{b} g$ vertex. 


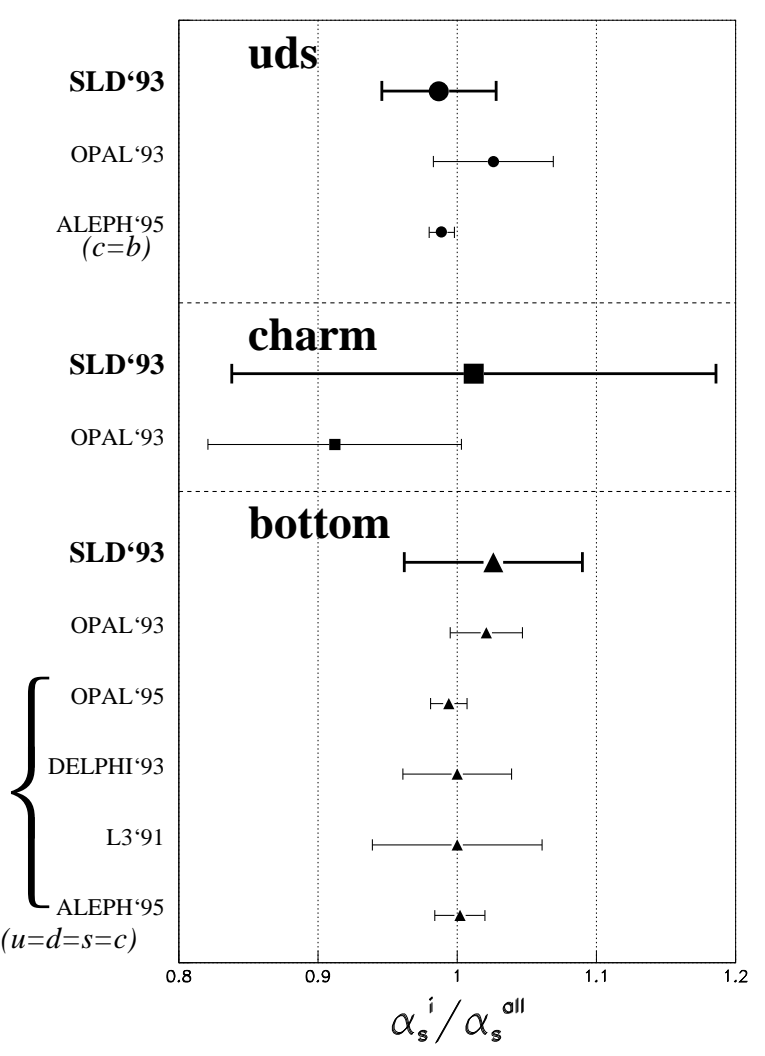

Figure 2.14: Values of $\alpha_{s}^{\text {flavors }} / \alpha_{s}^{\text {all }}$ previously measured values. The symbols $c=b$ and $u=d=s=c$ indicate assumptions of the flavor independence between these flavors. References for these values are provided in the text.

\section{Chapter 3}

\section{Experimental Apparatus}

\subsection{The SLAC Linear Collider (SLC)}

The SLAC Linear Collider (SLC) [75] accelerates both electrons and positrons up to $50 \mathrm{GeV}$. The SLC consists of mainly five systems: a polarized electron gun, damping rings, a linear accelerator (LINAC), arcs and a final focus system. The layout of the SLC is shown in Fig. 3.1.

Longitudinally polarized electrons are produced two times per $120 \mathrm{~Hz}$ accelerator repetition cycle by irradiating a GaAs semiconductor cathode with a circularly polarized laser. These electrons form two "bunches". Each bunch contains approximately $5 \times 10^{10}$ particles.

These bunches are accumulated in the North Damping Ring after being accelerated up to an energy of $1.19 \mathrm{GeV}$. The damping ring reduces the phase-space of particles in a bunch. After a large number of turns, they are extracted from the ring and accelerated in the LINAC.

Approximately after the first bunch is accelerated down two-thirds of the LINAC, the second electron bunch is delivered onto a target to produce positrons, which are sent back to the South Damping Ring through the Return Line to wait for the next accelerator cycle.

The electron and positron bunches, accelerated up to $46.7 \mathrm{GeV}$ energies in the LINAC, are divided into two opposing arcs by a bending magnet.

Three superconducting quadrupole magnets in the final focus system provide $2.6 \mu \mathrm{m}$ (horizontal) $\times 0.8 \mu m$ (vertical) flat beams and a stable Interaction Point (IP) where electrons and positrons collide [75]. 

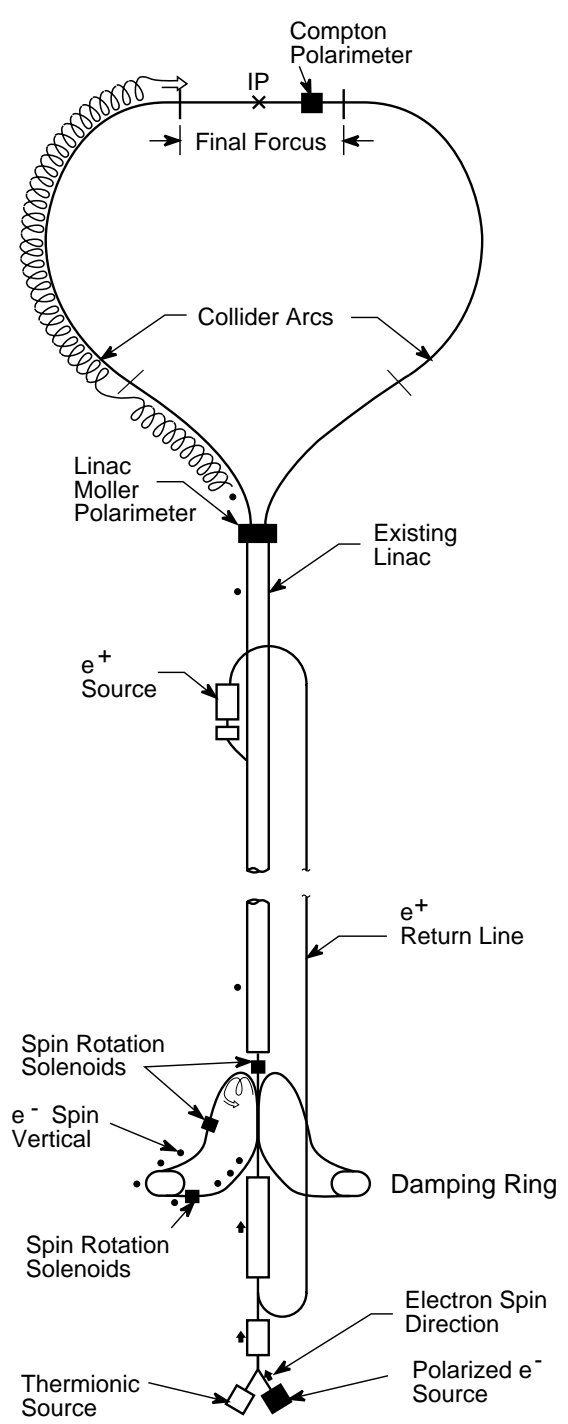

Figure 3.1: The layout of the SLAC Linear Collider [75].

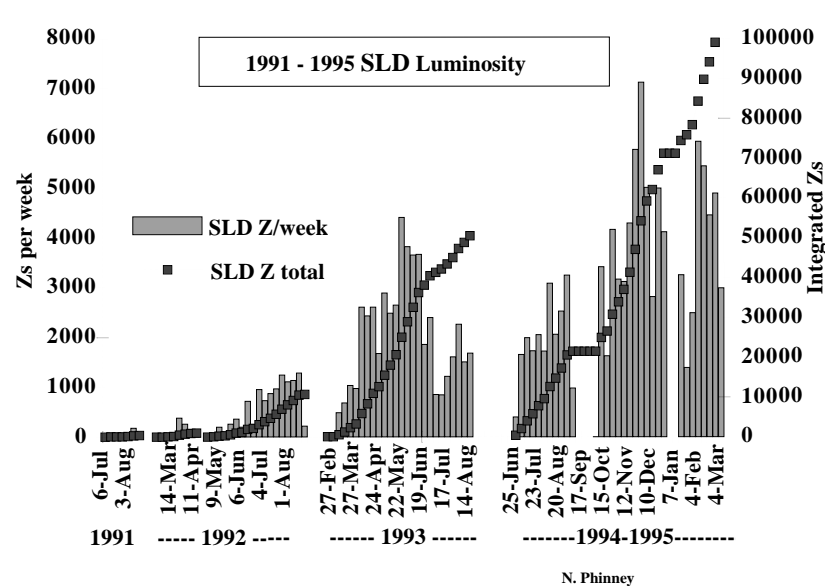

Figure 3.2: The history of the integrated luminosity for the 1991 through 1995 runs of the SLD experiment [76].

A number of upgrades were performed on the SLC between the 1993 and 1994-1995 runs. Introduction of an "over-compression" technique ${ }^{1}[77]$ allows a shorter bunch length and a reduced energy spread. A final focus optics upgrade was designed to reduce chromatic effects. A history of the integrated luminosity for the 1991 through 1995 runs of the SLD experiment is shown in Fig. 3.2.

\subsection{SLC Large Detector (SLD)}

The SLC Large Detector (SLD) [8] has recorded the $e^{+} e^{-}$annihilation events at the $Z^{0}$ resonance. The SLD is a cylindrically symmetric detector. Fig. 3.3 shows the cutaway and quadrant sections of the detector.

Charged tracks are measured in the Central Drift Chamber (CDC) [78] and in the Vertex Detector (VXD2) [79]. The Cherenkov Ring Imaging Detector (CRID) [80] makes identifica-

${ }^{1}$ The rf cavity in the dumping ring-to-LINAC (RTL) transport line compresses the longitudinal bunch distribution in a rectangular shape using the bunch energy-length correlation. 


\section{SLD}
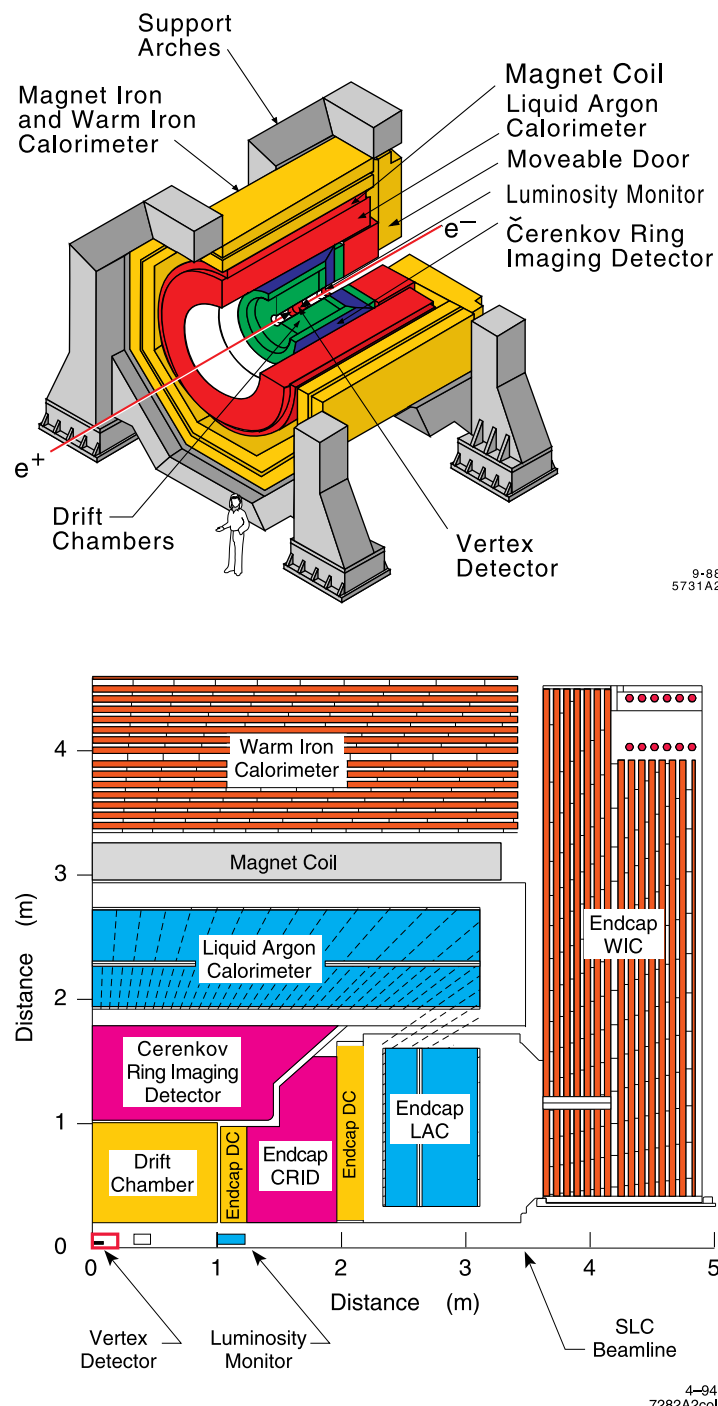

Figure 3.3: The cutaway and quadrant sections of the SLD [76] tion of the charged particles. Particle energies are measured in the Liquid Argon Calorimeter (LAC) [81]. The solenoidal coil provides a 0.6 T magnetic field for the measurement of charged particle momentum.

The SLD takes right-handed coordinate system. The $z$ coordinate is along the beam axis and points in the direction of the positron beam, with $z=0$ defined as the center of the CDC. The $x-y$ plane is perpendicular to the beam axis, with positive $y$ pointing vertically upwards, and positive $x$ pointing westwards. Cylindrical coordinates $(r \phi z)$ are also used with same $z$ axis of the $x y z$ coordinate system, where $r$ is the radial distance from the beam pipe and $\phi$ is measured in the $x-y$ plane counter-clockwise from the $x$ axis.

The subcomponents of the SLD are described briefly in the following sections.

\subsubsection{Vertex Detector (VXD2)}
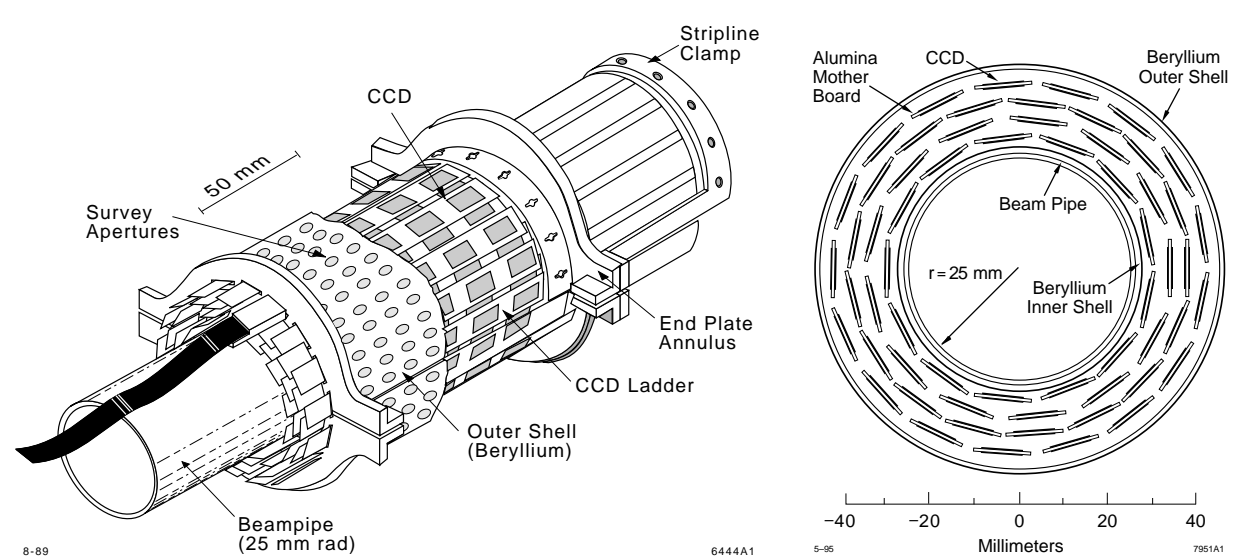

Figure 3.4: The SLD Vertex Detector, bird's-eye view (left) and $x y$ view (right).

The VXD2 [79] uses Charged Coupled Devices (CCDs). for detecting the deposition of ionization from traversing charged particles. Three dimensional precise track reconstruction is achieved by this pixel-based detector. The VXD2 consists of $480 \mathrm{CCDs}$ of $8.5 \mathrm{~mm} \times 12.7 \mathrm{~mm}$ die mounted on $9.2 \mathrm{~cm}$ long aluminum-ceramic boards (henceforth called ladders). Each ladder has 8 CCDs and composed of four cylindrical layers. The two inner layers are made 
up from 13 ladders and the two outer layers consist of 17 ladders. The CCDs are configured to cover the gaps between CCDs (Fig. 3.4). At least two hits are possible for tracks within $|\cos \theta|<0.75$, where $\theta$ is the polar angle respect to the beam axis. The vertex detector and the cryostat is clamped to each end of the CDC by means of a pair of aluminum support cones. The overall thermal dissipation in the detector is $12 W(0.1 \mu W$ per pixel $)$. The temperature of the detector has been maintained at $-80 \pm 2^{\circ} \mathrm{C}$ by the liquid nitrogen boil-off gas flow through cryostat cylinder made of expanded poly-urethane surrounding the VXD2 to suppress dark current and the loss of charge transfer efficiency.

Each CCD die contains $385 \times 578$ pixels which is a unit of Metal Oxide Semiconductor (MOS) structure of $n$-buried channel fabricated on $p$-type epitaxial layer having a $p^{+}$ substrate. Each pixel is $22 \mu \mathrm{m}$ square and has a depletion depth of $5 \mu \mathrm{m}$. Charge collection occurs over the thin epitaxial layer $(\sim 15 \mu \mathrm{m})$ which results in signal charge are very unlikely to be distorted due to $\delta$ electrons. A min-I particle traversing a CCD deposits about $80 e / \mu m \times 20 \mu m=1600 e$ of which about half will diffuse into the depletion region. The pixel threshold is set around 100e (thermal noise corresponds to 30e)

On receipt of a trigger all CCDs are read out in a raster-scan mode from the output node at the corner of CCD. A deposited charge is held statically in the potential well just below the surface of the MOS structure, typically $10 \mu \mathrm{m}$ in depth. These charges are transferred onto the CCD output node (one per CCD die) by the extra potential well provided by $10 \mathrm{~V}$ drive pulses. I-shifts which shifts all the charges in the CCD imaging (or I-) area down by one raw, with the bottom raw being moved into the Readout (or R-) register. There follow $385 R$-shifts which clock the charges in the R-register in turn onto the output node. The shaped pulse are digitized by a 10bit flash ADC synchronized to the $540 \mathrm{~ns}$ R-shift period.

Each pixel needs 4 bytes of storage for its address and contents. Since it is not feasible to store all of 120 million pixel data, a pipeline processor named Cluster Processor [82] was introduced to reduce the data size to a manageable level (20K bytes per event) selecting $3 \times 3$ hit clusters ${ }^{2}$.

${ }^{2}$ A Cluster Processor consists of 4 Application-Specific-Integrated-Circuits (ASIC) per CCD die. An ASIC consists of approximately 5000 gate logics. It worked synchronously equal to the CCD readout rate of $2 \mathrm{MHz}$. The $3 \times 3$ kernel size was taken into account charge diffusion in the undepleted part of the epitaxial layer. The backgrounds seen in the detector are predominantly from X-ray conversions and long tracks which, being nearly parallel to the beam direction.

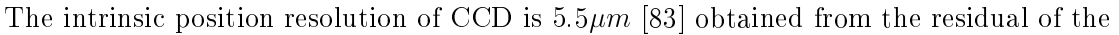
middle hit in a triplet of VXD hits from the same track on three separate layers. The average number of noise hits per CCD is 5 .

\subsubsection{Central Drift Chamber (CDC)}

The charged particle tracking is mainly carried out by the Central Drift Chamber(CDC) [78]. The CDC, which covers the barrel region, is $2 \mathrm{~m}$ long with an inner radius of $20 \mathrm{~cm}$ and an outer radius of $1.0 \mathrm{~m}$.

The structure of the drift cell is of the 'jet-chamber' type. The wire configuration is shown in Fig. 3.6. Each cell has an row of 8 sense wires of $25 \mu \mathrm{m}$ diameter gold-plated tungsten. The field shaping and guard wires are $150 \mu m$ diameter gold-plated aluminum. The CDC contains 640 cells organized into 10 superlayers. As the charged particle traverses the cell, a trail of ions is produced along the track trajectory in the drift gas. The deposited electrons will drift toward the sense wires $(7.9 \mu \mathrm{m} / \mathrm{ns})$ under the electric field $(0.9 \mathrm{kV} / \mathrm{cm})$ provided by the field shaping wires. From the measured time between a beam crossing and the arrival of the signal on the sense wire, one can determine the distance of a hit from the sense wire via a time-to-distance relation. The gas mixture in the $\mathrm{CDC}$ is $75 \% \mathrm{CO}_{2}, 21 \% \mathrm{Ar}, 4 \%$ isobutane, and $0.3 \% \mathrm{H}_{2} \mathrm{O} . \mathrm{CO}_{2}$ is chosen as the primary gas because of its low diffusion constant for drift electrons. Argon is added to increase the gas avalanche gain, Isobutane quenches photons from the avalanche that might cause fake second-hand ionization by the photoelectric effect, and water reduces the aging effect in the radiation environment of SLC by supressing dark current due to its electron affinity [84]. The CDC is maintained at a constant temperature of $20^{\circ} \mathrm{C}$ by a series of cooling tubes attached to each end-plate. No attempt is made to stabilize it against fluctuations in atmospheric pressure, however, the drift velocity is calibrated within $0.1 \%$ accuracy taking into account the pressure, temperature and water vapor concentration.

The gas-amplified charge is readout from both ends of the sense wires. At each beam crossing, the waveform of the charge deposited on each sense wire is sampled onto a switched capacitor array called an Analog Memory Unit (AMU) [85]. For triggered events the stored waveform from each end is digitized by 12-bit ADC and transported serially over optical fibers into a FAST-BUS Waveform Sampling Module. The waveform information allows 
us the close hit separation. An efficiency of $50 \%(\sim 100 \%)$ is achieved by a separation of $1 \mathrm{~mm}(1.5 \mathrm{~mm})$, which corresponds to approximately $3 \%$ of hits on tracks in hadronic $Z^{0}$ decays are too close to be resolved.

This double-ended readout, combined with the $330-\Omega$ resistance of the sense wires, permits a charge division measurement of the axial coordinate along with sense wire accurate to about $5 \mathrm{~cm}$. Furthermore, the superlayers alternate between Axial (A) and stereo layers which have a $\pm 41 \mathrm{mrad}$ stereo angle ( $\mathrm{U}$ and $\mathrm{V}$ ) with respect to the beam axis to allow a three dimensional measurement of the track trajectory.

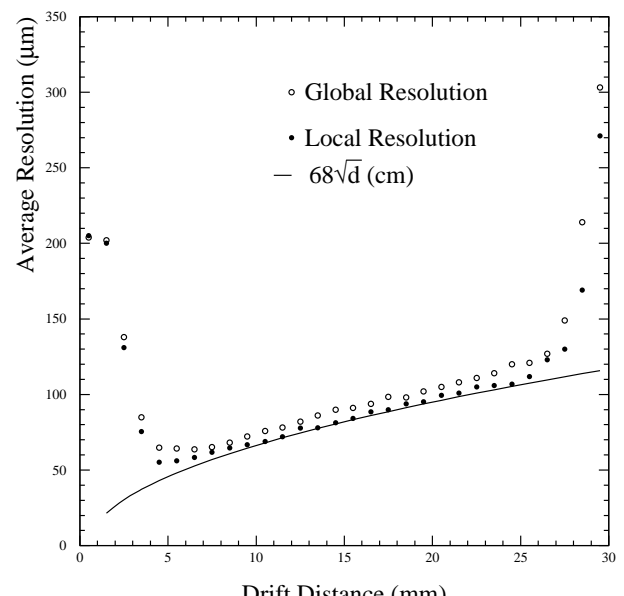

Figure 3.5: Drift distance resolution as a function of the drift distance in a cell [78]. The line indicates the contribution from the diffusion of drift electrons.

The intrinsic drift distance resolution averaged over the drift distance ${ }^{3}$ is $82 \mu m$ primarily dominated by the diffusion coefficients of the gas, the ratio of sampling speed to electron drift velocity in the gas, and electrical noise. The global resolution is measured to be $92 \mu \mathrm{m}$. the difference between these $(40 \mu \mathrm{m})$ reflects the global mis-alignment and the non-uniformity of the drift velocity near the sense and field wires (Fig. 3.5).

The overall tracking performance will be discussed in Sect. 3.4 .

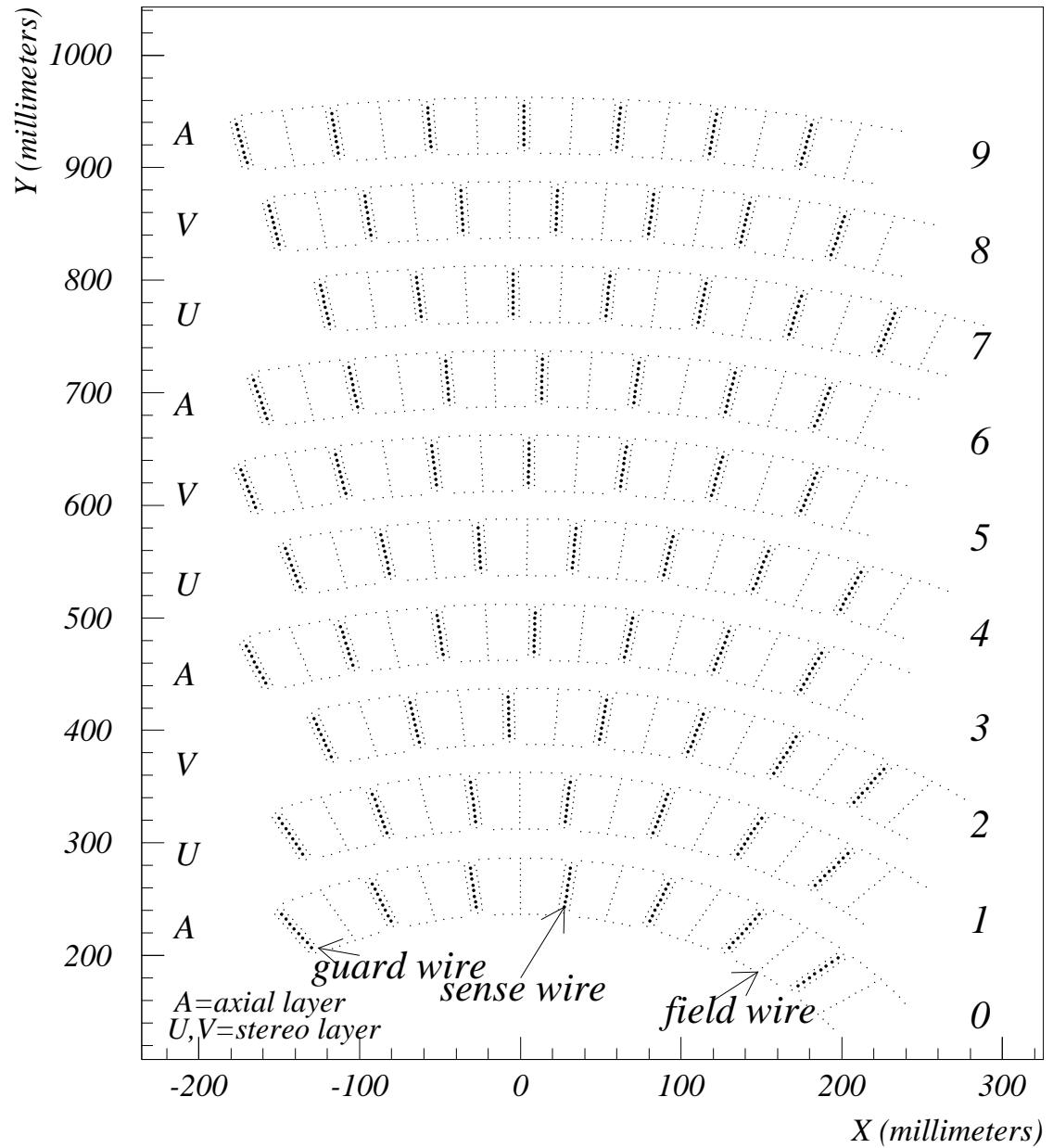

Figure 3.6: Wire configuration of the Central Drift Chamber (CDC). Axial layers are labeled 'A', while the stereo layers are labeled ' $U$ ' and ' $V$ ' [78].

${ }^{3}$ derived from Gaussian fit to the residual distribution in a given cell. 


\subsubsection{Cherenkov Ring Imaging Detector (CRID)}

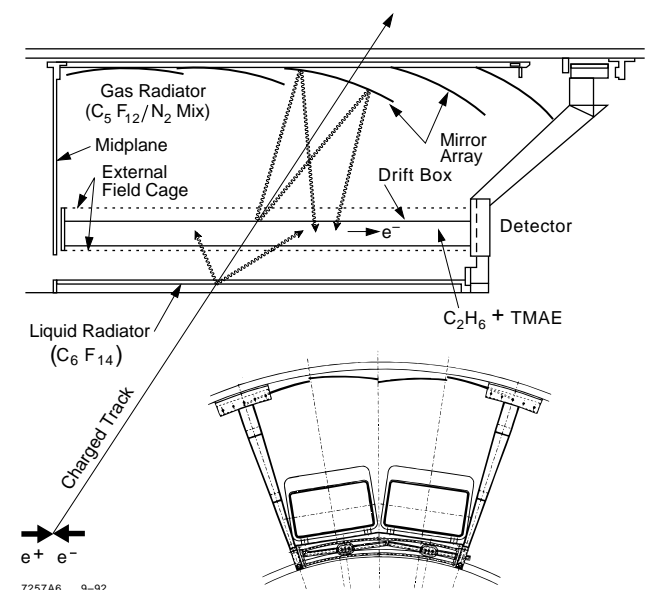

Figure 3.7: Schematic view of the Cherenkov Ring Imaging Detector(CRID)

The Cherenkov Ring Imaging Detector (CRID) [80] is designed to identify charged particles; $\pi / K / p$ separation is possible up to about $30 \mathrm{GeV} / \mathrm{c}$, and $e / \pi$ separation up to $6 \mathrm{GeV} / \mathrm{c}$. When the velocity of a charged particle exceeds the light velocity in a medium, Cherenkov light is emitted in a cone with an opening angle $\theta$ with respect to the direction of a charged particle, where $\cos \theta=1 / \beta n, \mathrm{n}$ is the refractive index of the medium and $\beta$ is the velocity of the particle in the light velocity unit. The combination of velocity measurement by this Cherenkov angle and momentum measurement by the tracking detectors makes possible particle identification.

The CRID employs liquid $\left(C_{6} F_{14}\right)$ and gas $\left(76 \% C_{5} F_{12}+24 \% N_{2}\right)$ radiators. Due to the different refractive indices, the former covers the low momentum $(0.3<p<5 \mathrm{GeV} / \mathrm{c})$ and the latter covers the high momentum region above $2.5 \mathrm{GeV} / \mathrm{c}$ [86].

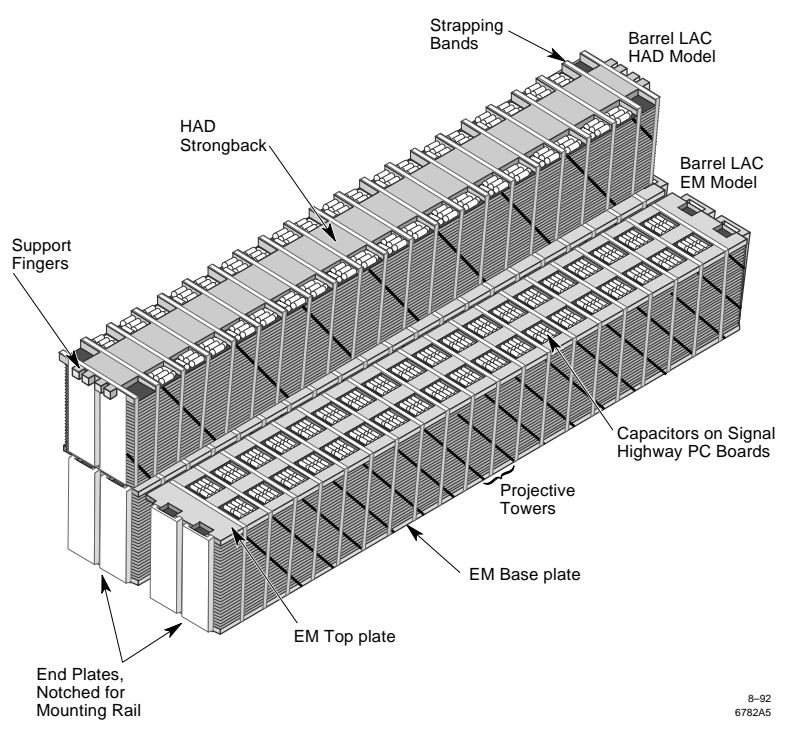

Figure 3.8: LAC barrel EM and HAD modules

\subsubsection{Liquid Argon Calorimeter(LAC)}

The Liquid Argon Calorimeter(LAC) [81] is composed of a cylindrical barrel calorimeter and two endcap calorimeters. The barrel LAC is $6 m$ long and extends in radius from 1.8 to $2.9 \mathrm{~m}$. The two endcaps fit just inside the barrel cylinder and are mounted on retractable end doors of the detector. The LAC provides both electromagnetic and hadronic calorimetry over the full azimuthal angle and polar angle $|\cos \theta|<0.98$ region (the barrel LAC covers $|\cos \theta|<0.84$, while the endcaps cover $0.82<|\cos \theta|<0.99)$.

The barrel section of the LAC is composed of 288 modules, and spanned in the azimuth direction $(\phi)$ by 48 modules of width $30 \mathrm{~cm}$. In the radial direction, two separate type of modules - electromagnetic (EM) and hadronic (HAD) - are stacked up. These modules are shown in figure 3.8

LAC modules are constructed as parallel plate liquid argon ionization chambers. The lead plates are grounded, while the tiles are held at high voltage and serve as the charge collecting electrodes. Transverse segmentation of the LAC is provided by the segmented lead 
tiles. Tiles from successive layers are laid out in a projective pattern, and stacks of these are ganged longitudinally into towers. The azimuthal range of the barrel is divided into 192 EM towers, the polar angle range of the barrel is divided into 34 EM towers. The transverse segmentation in the HAD sections is twice as coarse as in the EM sections. The EM sections which have a thickness of 21 radiation lengths contain approximately $99 \%$ of the energy of a $45 \mathrm{GeV}$ electron shower. The total EM + HAD thickness of 2.8 absorption lengths contains $80-90 \%$ of the energy of a hadron shower.

The endcap sections of the LAC are composed of 16 wedge shaped modules segmented in the azimuthal direction. The tower structure of endcap modules is similar to those of the barrel region. The energy resolution is estimated to be $\sigma(E) / E=12 \% / \sqrt{E}$ in the EM sections and $\sigma(E) / E=60 \% / \sqrt{E}$ for hadrons, respectively.

\subsubsection{The solenoidal coil}

The SLD magnet consists of a $5.9 \mathrm{~m}$ diameter, $6.4 \mathrm{~m}$ long and $29 \mathrm{~cm}$ thick normal conductive aluminum coil. It provides a magnetic field of 0.6 Tesla inside the coil. The field has been mapped within $0.05 \%$ accuracy over the tracking volume of the CDC to ensure the accurate measurement of momentum [8].

\subsubsection{Warm Iron Calorimeter (WIC)}

The Warm Iron Calorimeter (WIC) [87] plays four roles: measurement of hadronic energy which has leaked out of the LAC, tracking for muons, magnetic flux return for solenoidal coil, and the structural support.

The WIC is constructed in the form of an octagonal barrel with planar endcaps. Each barrel octant and endcap of the iron calorimeter consists of 14 iron plates of $5 \mathrm{~cm}$ thick, interleaved with sampling chambers incorporating external readout electrodes with plastic streamer tubes. Fig. 3.9 schematically shows the chamber arrangement in a typical barrel octant.

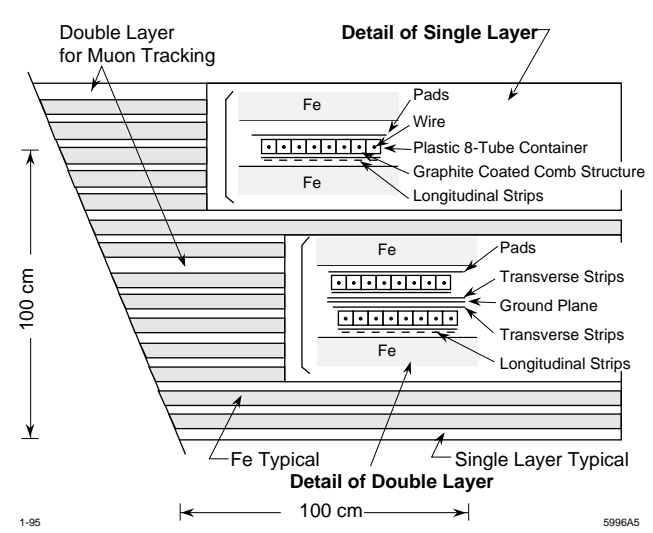

Figure 3.9: Warm Iron Calorimeter

\subsubsection{Luminosity Monitor (LUM)}

The Luminosity Monitor (LUM) [88] is a silicon-tungsten calorimeter designed to provide an absolute luminosity measurement by the rate of small angle Bhabha scattering. The LUM surrounds the beam pipe on both sides of the interaction point, and consists of two separate modules. The Medium Angle Silicon Calorimeter (MASiC) has 10 sampling layers of $1.74 X_{0}$ and the Luminosity-Monitor/Small-Angle Trigger (LMSTAT) has 23 sampling layers of $0.86 X_{0}$ mounted directly onto the final focus triplet, which cover $28-68 \mathrm{~m}$ rad and 68-190m rad, respectively (Fig. 3.10).

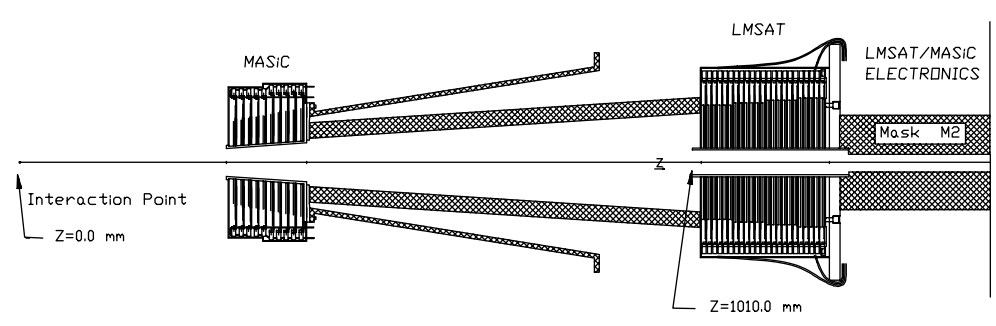

Figure 3.10: A side view of the Luminosity Monitor (LUM). 


\subsection{The SLD Monte Carlo Simulation}

A modern high energy physics detector such as SLD is a complex of sub-detector systems. It generally requires detailed computer simulation to understand its behavior thoroughly. Detector modelings implemented in the simulation provide information about acceptances, resolutions and efficiencies. It is also used to help choosing appropriate event selection and flavor tagging cuts. The SLD Monte Carlo simulation consists of two sequential steps; the event generation, and the detector simulation.

\subsubsection{Physics Simulation}

SLD uses the JETSET 7.4 event generator [56] to simulate the process of $e^{+} e^{-} \rightarrow$ hadrons at the $Z^{0}$ resonance. JETSET7.4 generates the parton shower according to the DGLAP equations [47] and string fragmentation for the hadronization, as described in Sect. 2.3. There is a variety of parameters to control the production of final state particles. These parameters were optimized to reproduce present SLD/LEP measurements. The major optimized parameters [89] are shown in Table. 3.1 along with the default sets.

\begin{tabular}{|c|c|c|}
\hline Parameter & Default & Optimized \\
\hline$\Lambda_{p s}$ & $0.29 \mathrm{GeV}$ & $0.26 \mathrm{GeV}$ \\
$Q_{0}$ & $1.0 \mathrm{GeV}$ & $1.0 \mathrm{GeV}$ \\
$\sigma_{q}$ & $0.36 \mathrm{GeV} / \mathrm{c}$ & $0.39 \mathrm{GeV} / \mathrm{c}$ \\
$\mathrm{a}$ & 0.3 & 0.18 \\
$\mathrm{~b}$ & $0.58 \mathrm{GeV}^{-2}$ & $0.35 \mathrm{GeV}^{-2}$ \\
\hline$\epsilon_{c}$ & -0.05 & -0.06 \\
$\epsilon_{b}$ & -0.005 & -0.006 \\
s quark prob. & 0.30 & 0.28 \\
\hline
\end{tabular}

Table 3.1: JETSET7.4 parameters: default and optimized values

Unstable particles in the final states are decayed by JETSET. For $B$ hadron decays, SLD incorporates an alternative program package implementing the CLEO model [90] based on the CLEO and ARGUS measurements [91]. The $B$ hadron lifetimes in the Monte Carlo simulation are set to $1.55 \mathrm{ps}$ for $B$ mesons and $1.10 \mathrm{ps}$ for $B$ baryons. The CLEO model was further tuned in the SLD environment [92].

\subsubsection{Detector Simulation}

The SLD detector simulation is performed using the package GEANT version 3.21 [93]. The modeling of detector materials for mechanical support, detection media, electronic cables, etc. provides the simulation of the effects such as energy loss, multiple scattering, gamma conversions and particle decays. Long-lived particles, like $K_{s}^{0}$ and $\Lambda^{0}$ may interact with the detector material before they decay. Their decays are treated separately in the GEANT simulation.

In addition, the effect of the beam-related backgrounds measured in random trigger events recorded during beam crossings is simulated by overlaying the event onto generated $\mathrm{MC}$ events. Other effects such as readout imperfections and electronics noise were also taken into account.

Finally, these fully simulated Monte Carlo events were reconstructed using the same processes and written in the same format as the real data events. 


\subsection{Tracking System Performance}

As will be seen in the next sections, a thorough understanding of the details of the tracking system is crucial to the analysis presented here. This section gives an overview of the excellent performance of the SLD tracking system and our understanding of its working. Below, we discuss the global performance of these detectors. As charged tracks are reconstructed using both the VXD and CDC, this global performance directly impacts the physics analyses that rely on the precision of the tracking system.

Knowledge of the interaction point is also essential to distinguish clearly the primary vertex from other vertices for the flavor tagging.

\subsubsection{Charged Track Reconstruction}

Charged track reconstruction begins with looking for segments of tracks in the individual drift cells of the CDC. The time and charge information from both ends of each wire are combined to yield the distance of each hit from its sense wire and its location along the wire. Hits from different wires within a cell are grouped into vector hits (VHs). A VH-finder searches for straight or slightly-curved strings of hits on the eight sense wires in each cell. At least 3 hits are required to form a vector hit. Each set of hits produces two possible VHs, one on either side of the sense wire plane. These vectors serve as the input to the pattern recognition phase, which attempts to find actual tracks in the CDC.

These VHs are linked to form arcs in the four axial layers, then vectors from the stereo layers are added if they fit on these arcs. The information from the charge division measurement is used to project the stereo vector hits onto arcs. In the first instance, only tracks with ten VHs (tracks hit all superlayers) are investigated. Among these, the one with the best $\chi^{2}$ is called a candidate track and its VHs are removed from further consideration. After identifying all tracks with ten VHs, the algorithm continues recursively until all tracks of at least three VHs are found.

Finally, all track candidates are processed by an iterative track fitter, taking into account the variation of the magnetic field, energy loss, and material in the chamber.

The output of the track fitter is a track defined by two sets of track parameters, one set evaluated at its innermost radius, one at its outermost. Extrapolation inward to the VXD or outward to the CRID, LAC, or WIC begins with these sets of track parameters.

To match the tracks reconstructed in the CDC to corresponding hits in the VXD, the tracks are extrapolated inward to the outermost layer of the VXD. Within an appropriate search region of the extrapolated track error, a Billoir fit ${ }^{4}[94]$ is performed for each possible combination to select those VXD hits which provide the best match to the track. The efficiency for linking "good CDC tracks" to hits in the VXD is approximately $96 \%$ above a momentum of $4 \mathrm{GeV} / \mathrm{c}$, and falls to $93 \%$ at the lowest momentum, as multiple scattering becomes more important [78].

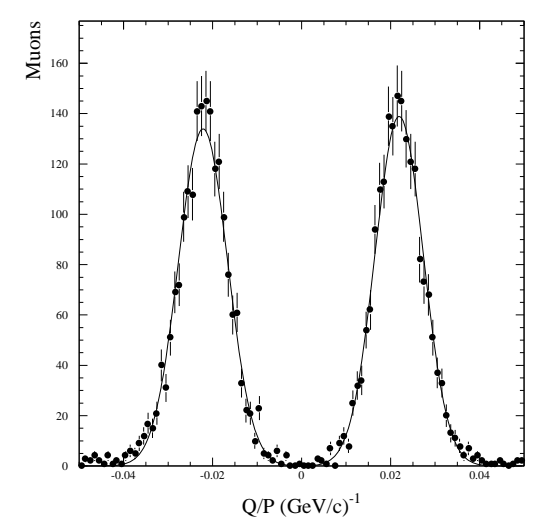

Figure 3.11: Distribution of charge over momentum for tracks from $Z^{0} \rightarrow \mu^{+} \mu^{-}$decays. The data are fit with one Gaussian for each peak [78].

The momentum resolution is parameterized by the formula:

$$
\begin{aligned}
\frac{\sigma\left(p_{t}\right)}{p_{t}} & =0.010 \oplus 0.0050 p_{t}(\mathrm{CDC} \text { only }) \\
\frac{\sigma\left(p_{t}\right)}{p_{t}} & =0.0095 \oplus 0.0026 p_{t}(\mathrm{CDC}+\mathrm{VXD} \text { combined })
\end{aligned}
$$

where $p_{t}$ is the track momentum perpendicular to the beam axis in $\mathrm{GeV} / \mathrm{c}[78,83]$. The first term is due to multiple scattering and the second one is the effect of measurement

\footnotetext{
${ }^{4} \mathrm{~A}$ Billoir fit makes use of the full $(n \times n)$ covariance matrix of the $n$ measurement points including
} multiple scattering effects in correlation terms. 
error (lever arm). The momentum resolution at high momentum was measured using the mono-energetic tracks in $Z^{0} \rightarrow \mu^{+} \mu^{-}$decays (Fig 3.11). At low momentum, cosmic rays passing through the CDC are used by considering the upper and lower halves of each track as two separate tracks.

\subsubsection{Impact Parameter Resolution}

The impact parameter of a track is its distance of closest approach to the IP. Its quality is determined by how well one can measure the position of the IP and how well one can extrapolate the tracks.

The error on the impact parameter can be expressed by the equation for the VXD and CDC tracking system [96]

$$
\begin{aligned}
\sigma_{\delta}^{r \phi} & =11 \oplus \frac{70}{p(\mathrm{GeV} / \mathrm{c}) \times \sin ^{\frac{3}{2}} \theta} \mu m \\
\sigma_{\delta}^{r z} & =38 \oplus \frac{70}{p(\mathrm{GeV} / \mathrm{c}) \times \sin ^{\frac{3}{2}} \theta} \mu m
\end{aligned}
$$

and is shown in Fig. 3.12

The former term is the intrinsic resolution of the tracking system and the latter is the multiple scattering term, where $\theta$ is the track polar angle and $p$ is the momentum of the charged particle. The intrinsic impact parameter resolution was measured from the miss distance of $\mu^{+} \mu^{-}$pairs at the IP (Fig. 3.13). These events provide pairs of isolated collinear tracks that have high momentum and minimum multiple scattering. The multiple scattering term was measured from hadronic $Z^{0}$ decays split into bins of momentum and $\cos \theta$. The width of a core Gaussian fit was taken.

Fits to CDC data alone yield $\sigma_{\delta}^{r \phi}=155 \mu \mathrm{m}$ and $\sigma_{\delta}^{r z}=1.9 \mathrm{~mm}$ at high momentum [78].

\subsubsection{Determination of the Interaction Point (IP)}

It is important to clearly distinguish secondary decay vertices from the IP. The extremely small luminous region of the SLC is a significant advantage compared with the LEP environment.
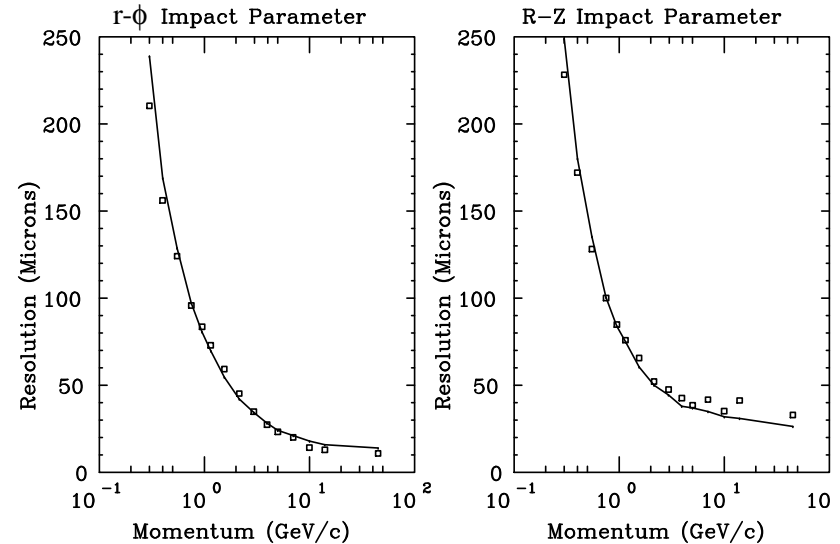

Figure 3.12: The impact parameter resolutions as a function of track momentum, for the $x y$ and $r z$ planes. The points are those from the data and the solid lines are the resolutions obtained from the MC [95].
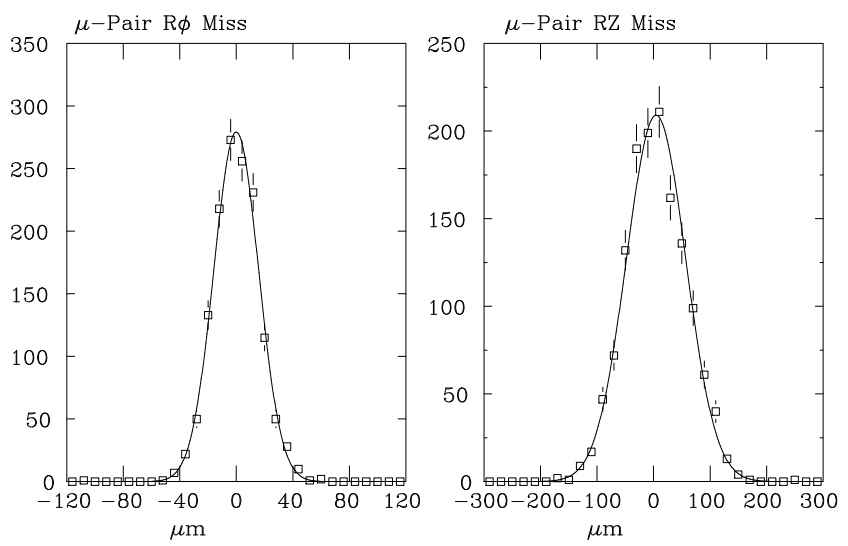

23.59:53 3.0N8

Figure 3.13: $\mu$-pair miss distance in $r \phi$ and $r z$ projections 


\section{Transverse Position}

The SLC luminous region is extremely small $(\sim 2.6 \mu \mathrm{m}$ in $x$ and $\sim 0.8 \mu \mathrm{m}$ in $y)$ [75]. Beam steering in the SLD final focus driven by feedback loops makes the beam position stable within $(\lesssim 6 \mu m)$ over the period of many $Z^{0}$ events [83, 97].

The spread in the interaction region is considerably better than, for instance, $120-$ $150 \mu m \times 5 \mu m$ in the typical LEP environment [98], where one may attempt to find the primary vertex (PV) from fitting all of the tracks event-by-event. The error has an ellipse shape due to the inevitable presence of collimated jets of particles. This is shown schematically in Fig. 3.14(a). Typically, the size of the errors are $100 \mu \mathrm{m}$ along the ellipse major axis, and $15 \mu \mathrm{m}$ along the ellipse minor axis. At the LEP environment, one might suffer from the large hemisphere correlation for the determination of the IP along the jet axis.

Thanks to the extremely small and stable luminous region, we can substitute it by the averaged position of the SLC IP rather than attempt to find the PV event-by-event. The error ellipse on the mean IP will be roughly circular. The possible bias in the PV position due to mis-reconstructed tracks and long-lived heavy hadrons will be greatly reduced.

To find values for the average IP, $<\mathrm{IP}>$, during the entire run, the SLD data sample is divided into sets of roughly 30 sequential hadronic decays. The $\langle\mathrm{IP}\rangle$ for each set is derived by fitting all tracks which have VXD hits and which come within $3 \sigma$ of the trial $<\mathrm{IP}>$ to a common vertex. This process is iterated until it converges. Typically, 330 tracks are used in a fit. Information from the SLC correctors is used to help determine exactly when a major shift occurs. When a major shift is found within a set, the boundaries of the set are changed to coincide with where the IP shift occurs.

The Uncertainty in the $\langle\mathrm{IP}\rangle,\left(\sigma_{I P}\right)$, is the combination of the statistical error from the fit $(\sim 3 \mu \mathrm{m})$, the extent of the SLC luminous region $(\sim 1 \mu \mathrm{m})$, and the motion of the IP within a set $(\sim 6 \mu \mathrm{m})$. This totals $\sim 7 \mu \mathrm{m}$ when added in quadrature.

The distribution of track impact parameters with respect to the $<\mathrm{IP}>$ in $Z^{0} \rightarrow \mu^{+} \mu^{-}$ events (Fig.3.14(b)) can provide an independent check on the precision of the beam position measurement, because those events are not used in the determination of the $<\mathrm{IP}>$. The $\sigma$ of the distribution of $12.7 \mu \mathrm{m}$ implies $\sigma_{I P}=6.7 \mu \mathrm{m}$ after the extrapolation error is subtracted in quadrature, which confirms that our estimates of the uncertainties on $<\mathrm{IP}>$ [83].

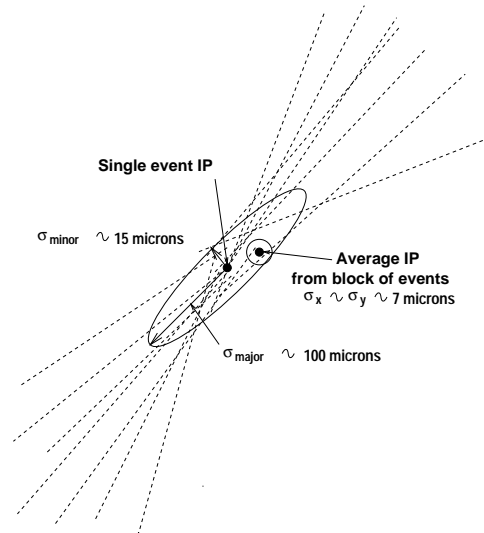

(a) The difference in the transverse IP position and errors determined from a single event and the average over a block of events [99].

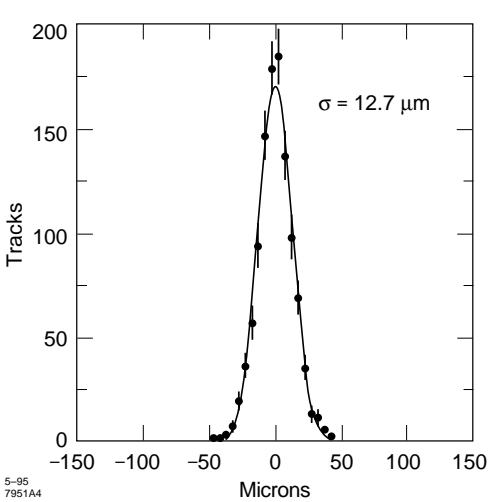

(b) Distribution of the track impact parameters in $\mu^{+} \mu^{-}$events with respect to the averaged IP determined from hadronic events [83]. The line shows the Gaussian fit to the data (plot). 


\section{Longitudinal Position}

The averaged IP along beam line cannot provide an adequate estimate of the event PV position due to the rather large beam interaction region $z$ spread of $\sigma_{z} \sim 700 \mu m$. The best estimate of the $\mathrm{PV} z$ position for each event comes from a technique using only the median $z$ of the tracks in the event itself. Each track with associated VXD hits is extrapolated to the point of closest approach to the $<\mathrm{IP}>$ in the $x-y$ plane, and the $z$ coordinate of the track at this point is denoted as $z_{\text {doca }}$. The event IP $z$ location is defined as the median of the $z_{\text {doca }}$ values from the selected tracks within $3 \sigma$ of the $x-y<\mathrm{IP}>$ position. The choice of the median $z$ method instead of the more common approach involving vertex finding is more robust against the distortion due to the inclusion of tracks not originating from PV. The typical resolution for locating the PV $z$ as derived from MC are $(32,36,52) \mu m$ for $(u d s$, $c, b)$ events [83].

\section{Chapter 4}

\section{Event Selection and Flavor Tagging}

The hadronic $Z^{0}$ decay $\left(Z^{0} \rightarrow q \bar{q}(g)\right)$ manifests itself as jets of hadrons. The signature of hadronic events is that a large number of charged particles $(\sim 20)$ are detected in tracking devices, and a large fraction of the center-of-mass energy is deposited in the tracking devices and calorimeters.

First, the trigger conditions necessary to record data are discussed. The criteria for selecting hadronic event sample are then described. Next, the flavor-tagging technique based on the recently developed topological vertexing [100], and lifetime tagging are explained. Lastly, the criteria used to classify selected hadronic event sample into flavor tagged subsamples are discussed.

This analysis predominantly used charged tracks measured in the CDC and VXD. Calorimeter information was also used mainly in the event filtering. A set of cuts was applied to the data to select well-measured tracks and events well-contained within the detector acceptance in the barrel region. The tracking capability of the CDC and the precise CCD Vertex Detector, combined with the stable, micron-sized beam interaction point (IP), allows us to select $Z^{0} \rightarrow \bar{b}(g)$ and $Z^{0} \rightarrow q_{l} \bar{q}_{l}(g)\left(q_{l}=u, d, s\right)$ events using their quark decay topological signatures with high efficiency and purity, and with low bias against 3 -jet events, an important advantage of this analysis.

The analysis presented in this thesis is based on the 150,000 hadronic $Z^{0}$ decays collected between 1993 and 1995 with the VXD2 detector. 


\subsection{Hadronic Event Selection}

Data accumulation begins with sets of online triggers. The triggered events were read out from each sub-detector system and recorded onto magnetic tapes in the raw-data format. An off-line filter was then applied to pre-process the accumulated data in order to separate signal from the background before the full reconstruction was performed. Finally, for the purpose of this analysis, a set of selection criteria was applied to the fully reconstructed data sample in order to select events well-contained within the detector acceptance of the barrel region.

There are two kinds of backgrounds to be considered; the physics related background and beam-related background. The physics related background mainly consists of $Z^{0} \rightarrow \tau^{+} \tau^{-}$ decay and photon-photon collision radiated from beam bunches. The decay modes of $\tau$ lepton are dominated by so called 1-prong decay containing one charged track $(85.90 \%)$ such as $\tau^{-} \rightarrow l^{-} \bar{\nu}_{l} \nu_{\tau}(l=e, \mu)$ or $\tau^{-} \rightarrow \pi^{-}\left(\pi^{0} s\right) \nu_{\tau}$, and the 3-prong decay containing three charged tracks (14.01\%) [20]. Therefore $Z^{0} \rightarrow \tau^{+} \tau^{-}$decay typically yields 2 , 4, or 6 number of charged tracks per event. As for two photon collision, the cross section is very small compared to $e^{+} e^{-} \rightarrow Z^{0} \rightarrow$ hadrons (Fig. 2.8), and hadrons from this process mainly escape inside the beam pipe, and hence this background is almost negligible in the barrel region. The beam-related backgrounds are such as SLC-induced muons and/or synchrotron radiation.

\subsubsection{Event Trigger}

The SLD trigger is deigned to record $Z^{0}$ events with a high efficiency, while rejecting most of the beam-related background [101]

\section{The Energy Trigger}

This trigger is designed to record events with a large amount of energy in the LAC, and requires at least $8 \mathrm{GeV}$ total deposited energy for the EM and/or HAD calorimeter towers in the LAC. The thresholds, above which the energy deposit in calorimeter towers are recorded, are defined to be $60 \mathrm{ADC}$ counts for the EM towers (corresponding to $246 \mathrm{MeV}$ ) and $120 \mathrm{ADC}$ counts for the HAD towers (corresponding to $1.298 \mathrm{GeV}$ ).
2. The Tracking Trigger

CDC cells are marked as hit if 6 of 8 sense wires have pulses above threshold. A hit cell pattern corresponding to a $p_{t}>250 \mathrm{MeV} / \mathrm{c}$ charged track coming from the interaction point is required. At least two charged tracks are required to pass through 9 of 10 $\mathrm{CDC}$ layers and be separated from each other by at least $120^{\circ}$. All subsystems are read out.

3. The Hadron Trigger:

This trigger requires at least one charged track passing through 9 super layers and at least $2 \mathrm{GeV}$ energy deposit in the LAC. All subsystems are read out.

4. The Wide Angle Bhabha Trigger:

This trigger is designed to ensure all wide-angle Bhabha events (and $Z^{0} \rightarrow e^{+} e^{-}$) are recorded. It requires a minimum total energy of $15 \mathrm{GeV}$ deposited in the LAC EM section. All subsystems are read out.

5. The Muon-pair Trigger:

This trigger requires at least one charged track in the event to satisfy the 9 CDC layer and two back-to-back barrel WIC tracks. All subsystems are read out.

6. The Bhabha Trigger:

This trigger requires a total energy in LUM EM2 section to be above $12.5 \mathrm{GeV}$ in both north and south detectors, where the sum is made over those towers above the threshold $(125 \mathrm{MeV})$. Only LUM systems are read out by this trigger.

7. The Random Trigger:

This trigger recorded data every 20 seconds, regardless of the detector status, to provide information for background studies.

At SLD, the efficiency for triggering on hadronic $Z^{0}$ decays approaches $100 \%$. The trigger rate is typically between 0.1 and $1 \mathrm{~Hz}$. The triggered events were written onto tape. 


\section{Trigger Veto}

It is important to inhibit to read out the detector on a condition of unexpected beamassociated background due to the variable noise environment of SLC such as SLC-induced muons and/or synchrotron radiation.

SLC-induced muons are created by photo-production from primary beam particles hitting an aperture upstream of the IP. As the final focus tunnel needs to be large enough to access the beam-line, there is not sufficient material present to absorb all of these muons. Hence, the muons can travel from their production point along the beam-line and can penetrate the detector.

The SLC produces copious synchrotron radiation from the focusing elements along the beam-line. A masking scheme around the IP shields sensitive portions of the SLD from the intense synchrotron radiation produced by the final dipoles and quadrapole triplet. Particles in the tail of the beam, however, can create synchrotron radiation which strikes the masks directly and can re-scatter into the detector, causing unwanted noise. Such events could be a significant source of dead-time when they are frequent, as it takes longer to read out SLD when its occupancy is high ${ }^{1}$.

Each trigger has vetoes to reject clearly unusable events. The track trigger, for instance, will be vetoed if the CDC has more than 275 hit cells out of the 640 cells ${ }^{2}$. During 1993, the track trigger veto was improperly configured for roughly 10\% of the 1993-1995 total data sample. During this hardware failure the trigger system accidentaly counted not only the normal hit cells but also the non-existent cells which correspond to the shorted motherboard of readout electronics as always being hit. This caused a wrong offset of $\sim 80$ cells for the number of hit CDC cells [102]. This bogus veto inhibited CDC from reading out high-multiplicity events. This is unfortunate for the analysis of 3 -jet events, which have a higher average multiplicity than 2-jet events, and are more likely to be affected by this veto. The cell overflow veto is simulated in the Monte Carlo, however, it is more prudent to omit this period since the event selection efficiency (which will be descibed in Sect. 4.1.3) for 3-jet events is estimated to be about $10 \%$ smaller than that of the normal run period, and thus the bias against 3-jet event is significant in this flawed sample.

\footnotetext{
${ }^{1}$ VXD2 takes $160 \mathrm{~ms}$ to be read out which correspond to 19 beam crossing equivalent time

${ }^{2}$ The average cell occupancy of the CDC is about 160 out of a total of 640 cells.
}

\subsubsection{Hadronic Event Filter}

Raw data recorded on tapes were processed to select $Z^{0}$ candidates and reject a large fraction of the background. In order to be quick and efficient, the initial event filtering was done using calorimeter information alone by requiring that an event deposit a certain amount of energy in the LAC and have good forward-backward momentum balance. This filter called 'EIT pass-1' [101] requires the following conditions.

1. $N E M H I>10$

NEMHI is the number of LAC EM towers with signals above 60ADC counts.

2. $E H I>15 \mathrm{GeV}$

$E H I$ is the sum of the energy deposited in all EM (HAD) towers with signals greater than a high threshold of at least 60 (120)ADC counts which is equivalent to $246 \mathrm{MeV}$ (1.298 $\mathrm{GeV})$.

\section{3. $E L O<140 \mathrm{GeV}$}

ELO is the sum of the energy deposited in all EM (HAD) towers with signals greater than a low threshold of 8 (12) ADC counts which is equivalent to $33 \mathrm{MeV}(130 \mathrm{MeV})$.

4. $2 \times E H I>3 \times(E L O-70)$

5. Both hemispheres divided at $z=0$ of the detector must have NEMHI $>0$

Cuts $2-5$ remove beam related background such as beam-wall events. The hadronic events are well-contained within the contour (2-4) on the EHI-ELO correlation [101].

The events which passed this filter were fully reconstructed to make a data summary tape (DST) containing information such as track helix parameters. The efficiency for hadronic events that pass this filter was estimated to be above $96 \%$ independent of quark flavor [103].

\subsubsection{Event Selection}

We apply a set of cuts designed to select events contained within the fiducial region of the SLD detector. Since we use charged tracks as a basis of the analysis, this restricts us to 
the barrel region of the SLD, as the CDC starts to loose tracking efficiency for $|\cos \theta| \geq 0.8$, where $\theta$ is the polar angle with respect to the beam axis. We also wish the energy flow of the event to be well contained within this fiducial region so as to guarantee that we are not missing large portions of the event that have ended up in the detector endcap.

We first define a "well measured track" as any track satisfying:

- $p_{t}>150 \mathrm{MeV} / \mathrm{c}$

- $|\cos \theta|<0.8$

- $|r|<5 \mathrm{~cm}$

- $|z|<10 \mathrm{~cm}$

where $p_{t}$ is the transverse momentum relative to the beam axis, $\theta$ is the polar angle with respect to the beam axis, $r$ is a distance of closest approach transverse to the beam axis and $z$ is a distance of closest approach along the beam axis. The cut on $p_{t}$ rejects most of tracks produced from beam-related background and from photon conversion, and loop-back tracks that have very low transverse momentum. Furthermore, the precise modeling of low $p_{t}$ track is difficult due to multiple scattering. The cut on $\theta$ is chosen to ensure that the tracks are well contained in the active region of the CDC. The cuts on $r$ and $z$ ensure that the tracks originate from the region of the interaction point.

The selected events for this analysis were required to satisfy:

- $\left|\cos \theta_{\text {thrust }}\right|<0.71$

- $\geq 7$ well measured tracks

- $E_{v i s}>20 \mathrm{GeV}$

- $\geq 3$ flavor tagging tracks

where $\theta_{\text {thrust }}$ is the polar angle with respect to the beam axis of the thrust axis $\vec{t}$ [33], which is defined to maximize the longitudinal momentum flow:

$$
T=\frac{\Sigma_{i}\left|\overrightarrow{p_{i}} \cdot \vec{t}\right|}{\Sigma_{i} \overrightarrow{p_{i}}}
$$

The thrust axis was calculated using calorimeter clusters. The requirement of $\geq 7$ well measured tracks eliminates physics related backgounds, especially $Z^{0} \rightarrow \tau^{+} \tau^{-}$decays. $E_{v i s}$ is the total energy calculated from the selected charged tracks assuming all tracks to be pions. The requirement for the flavor tagging tracks linked to the vertex detector, which will be defined in Sect. 4.2.1, ensures that the selected events can be used for flavor tagging. Figure 4.1 show the data sample before relevant cuts, with the Monte Carlo hadronic events overlaid. After applying these cuts, 77857 events remain.

Table 4.1 shows the efficiency for the different event flavors to pass hadronic event selection cuts. The efficiencies for 3-jet and heavy quark events are slightly higher than those of 2 jet and light flavors due to their higher multiplicities. Any small flavor bias in event selection will be explicitly corrected in the analysis, discussed in later section 5.3.

Table 4.1: The efficiencies for hadronic events of different primary quark flavors to pass the event selection cuts.

\begin{tabular}{|c|c|c|c|}
\hline & uds & charm & bottom \\
\hline 2-jet & $59.1 \%$ & 60.0 & 60.3 \\
$\geq 3$-jet & 61.7 & 62.2 & 62.4 \\
\hline
\end{tabular}

The non-hadronic event background contamination in the selected sample was estimated to be less than $0.20 \%$ from the MC, dominated by $\tau^{+} \tau^{-}$pairs [83]. 


\subsection{Flavor Tagging}

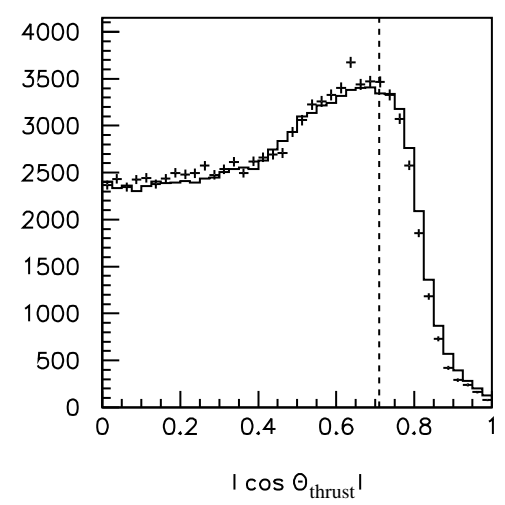

(a) $\cos \theta_{\text {thrust }}$ distribution

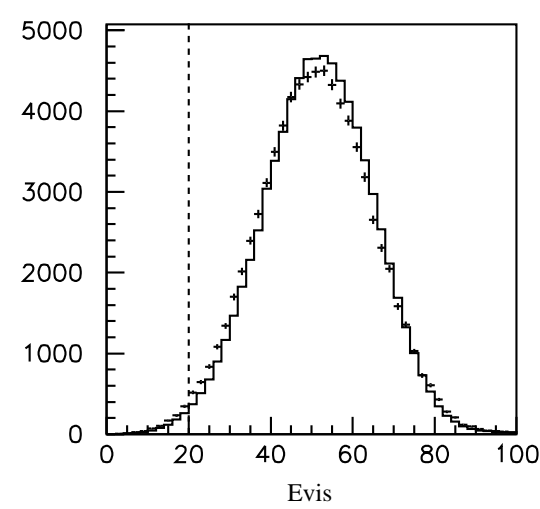

(c) Visible Energy distribution

Figure 4.1: Distributions of the event selection variables for data (points) and MC hadronic events (histograms). All of the other cuts have been applied except for the one being plotted. The dashed lines indicate the values of the cut on each variable.
Separation of the accepted event sample into tagged flavor sub-samples was based on the invariant mass of topologically-reconstructed long-lived heavy-hadron decay vertices, as well as on charged-track impact parameters in the plane normal to the beamline.

\subsubsection{Flavor Tagging Quality Track Selection}

In each event a jet structure was defined as a basis for flavor-tagging by applying the JADE jet-finding algorithm [68] to the 'well-measured tracks' described in the previous section; a value of the normalized jet-jet invariant-mass parameter $y_{c u t}=0.02$ was used. Charged tracks used for the subsequent event flavor-tagging were further required to have additional information from the VXD to select 'flavor tagging quality track's, which have more accurate track trajectories. 'Flavor tagging track's are required to satisfy :

- at least one VXD hit

Tracks that fail to link to a VXD hit will have poor extrapolation resolution at the IP.

- at least $40 \mathrm{CDC}$ hits

Tracks with few hits are likely segments of a kinked track, a track that curved back towards the beam-line before traversing the full radius of the CDC, a track from the decay of a very long lived neutral particle, or a track that has hits spoiled by another track at the track finding stage.

- the first $\mathrm{CDC}$ hit radius must be less than $39 \mathrm{~cm}$

As the extrapolation distance increases, the uncertainty on the track parameters increases and becomes more likely to link to fake VXD hits.

- quality of the CDC+VXD combined fit $\chi_{C D C+V X D}^{2}<5.0$

To reduce the possibility of having an incorrect vertex detector hit linked to the CDC track.

- $|P|>0.5 \mathrm{GeV}$

Low momentum tracks can suffer from large multiple scattering. 
- $\operatorname{doca}_{x y}^{I P}<0.3 \mathrm{~cm}$ and $d o c a_{r z}^{I P}<1.5 \mathrm{~cm}$

where $d_{o c a_{x y}^{I P}}^{I P}$ and $\operatorname{doca}_{r z}^{I P}$ are the distance of closest approach to the IP in the $x y$ and $r z$ plane, respectively. To remove tracks from interactions with detector material, a constraint was placed on the apparent origin of the track. This cut effectively eliminates strange particle decays.

- an error on doca $_{x y}^{I P}<250 \mu m$

This also eliminates poorly measured tracks.

- $V^{0}$ rejection

Track candidates from long lived neutral particle decays $\left(K^{0}\right.$ and $\left.\Lambda\right)$ and $\gamma$ conversions were rejected. Details are shown in Appendix A.

Fig. 4.2 shows the distributions of these variables for data and MC, with all cuts applied except the one being examined.

The impact parameter $[104,105]$ of each track, $\delta$, was given a positive (negative) sign according to whether the point-of-closest approach to its jet axis was on the same side (opposite side) of the IP as the jet in the $x y$ plane (see Fig. 4.3). The impact parameter of the track $Y$ is related to the lifetime, $\tau$, of its parent particle $X$ :

$$
\delta=\beta \gamma c \tau \sin \psi \sin \theta \propto c \tau
$$

where $c$ is the light velocity, $\theta$ is the polar angle of particle $X$ respect to the beam axis, $\psi$ is the angle between the directions of particle $X$ and $Y$. $\beta$ is the velocity of the particle $X$ and $\gamma=\left(1-\beta^{2}\right)^{-1 / 2}$ is Lorentz factor in the laboratory frame. Fig. 4.4 shows the distribution of the impact parameter normalized by its error, $\sigma_{\delta}$. This asymmetric distribution arises from the tracks from heavy hadrons which tend to populate the region of large positive values of $\delta$ due to long lifetime of the heavy hadrons and large acquired transverse momentum with respect to the flight direction of heavy hadrons.

The negative $\delta / \sigma_{\delta}$ region is the result of tracking resolution, interactions in the detector material, and IP position resolution. Therefore, this region is used to test the MC resolution modeling by comparing MC and data; our data are well described by MC.
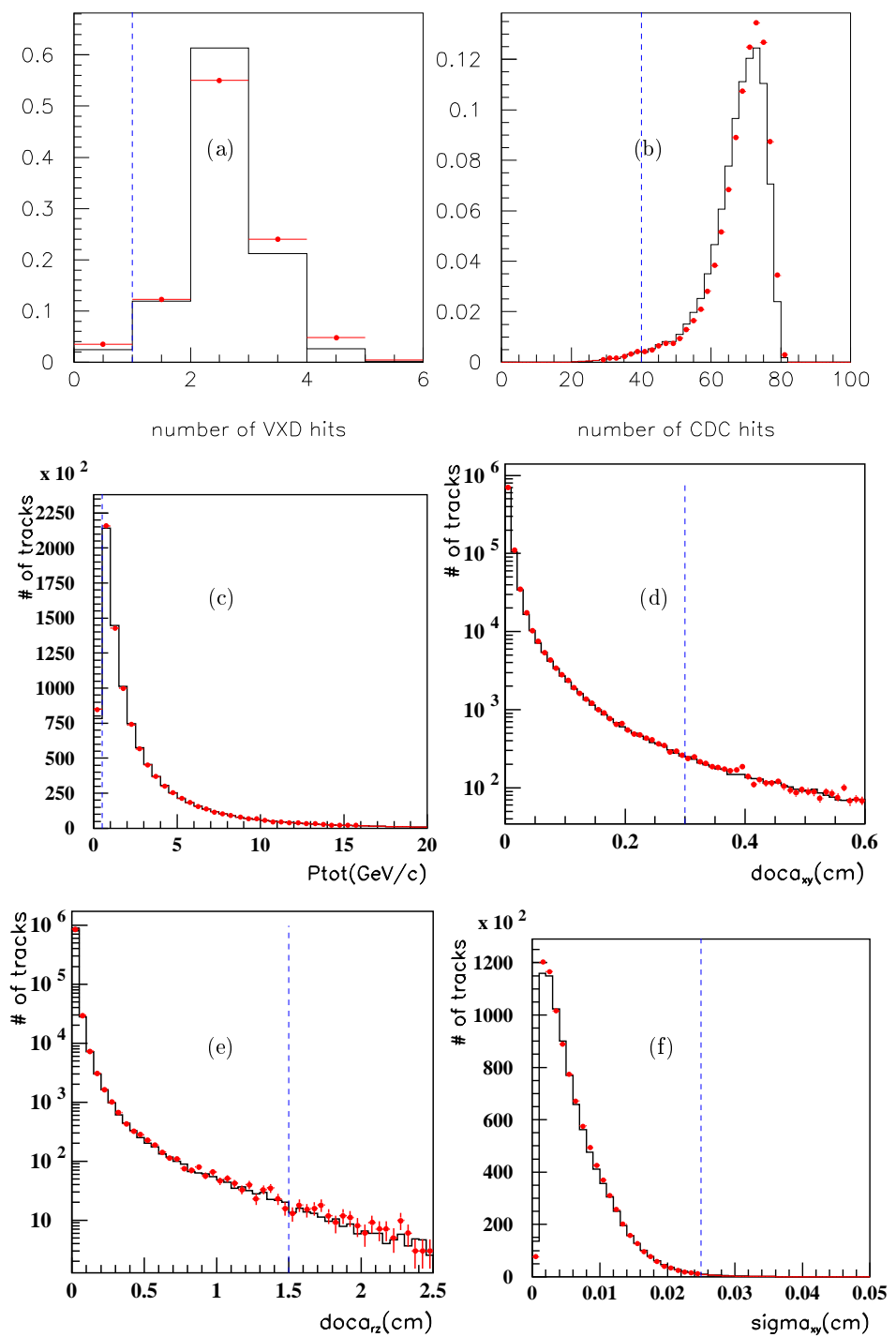

Figure 4.2: Distributions of the variables upon which cuts are applied to select flavor tagging quality tracks. All of the other cuts have been applied except for the one being plotted. The dashed lines indicate the values of the cut on each variable. 
(b)

(a)
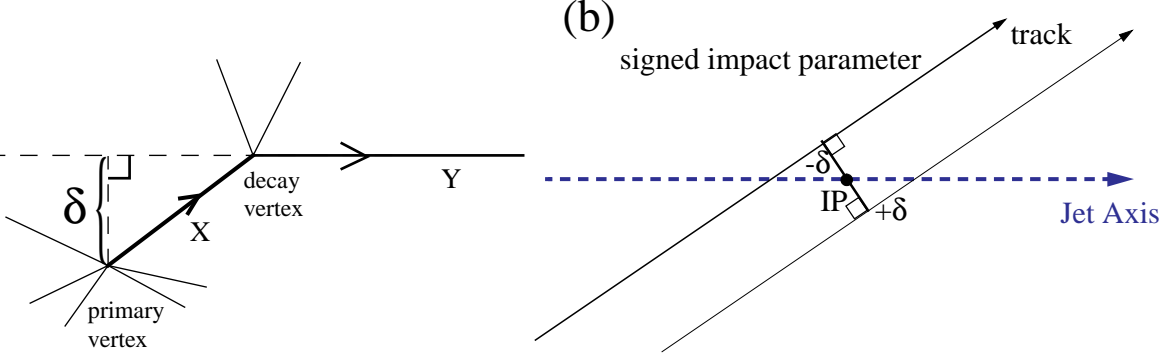

Figure 4.3: (a): Impact parameter $\delta$ for a track, $Y$, from a decay vertex [106]. (b): signing scheme of the impact parameter. Tracks which cross the jet axis downstream (upstream) of the IP are given positive (negative) impact parameters.

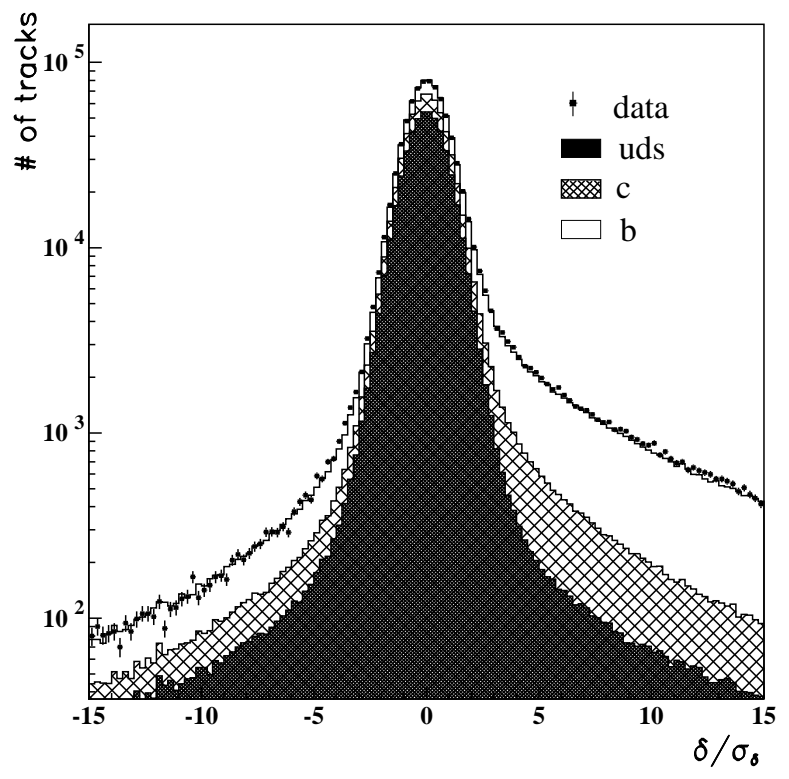

Figure 4.4: The normalized impact parameter distribution.

\subsubsection{Topological Vertex Mass}

In each jet we then searched for a secondary vertex (SV), namely a vertex spatially separated from the measured IP. In the search those tracks were considered that were assigned to the jet by the jet-finder. Individual track probability functions in 3-dimensional coordinate space were examined and a candidate SV was defined by a region of high track overlap density.

The method is described in detail in [100]; the philosophy adopted here is to search for the probability of vertices $V(\mathbf{r})$ in $3 \mathrm{D}$ coordinate space from track trajectories rather than by forming vertices from track combinations. At location $\mathbf{r}$, a Gaussian probability tube for a track trajectory, $f(\mathbf{r})$, is constructed from the helix parameter

$$
f(\mathbf{r})=\exp \left\{-\frac{1}{2}\left[\left(\frac{x-\left(x_{0}+y^{2} \kappa\right)}{\sigma_{T}}\right)^{2}+\left(\frac{z-\left(z_{0}+\tan (\lambda) y\right)}{\sigma_{L}}\right)^{2}\right]\right\}
$$

where the $x y$ coordinates have been transformed into $x^{\prime} y^{\prime}$ for each track (shown in Fig. 4.5), the $y^{\prime}$ coordinate is parallel to the track momentum vector at the point of closest approach to the IP $\left(\mathbf{r}_{\mathbf{0}}\right)$. The first term inside the exponential includes a parabolic approximation to the circular track trajectory in the $x y$ plane. The parameter $\kappa$ is determined from the particle charge and momentum, and the SLD magnetic field. The second term describes the propagation of the trajectory in the $z$ direction, and $\lambda$ is the dip angle of the track with respect to the beam axis. Parameters $\sigma_{T}$ and $\sigma_{L}$ are the measurement errors for the track at point $\mathbf{r}_{0}$

The relative probability of a vertex at $\mathbf{r}$ is defined as:

$$
V(\mathbf{r})=\sum_{i} f_{i}(\mathbf{r})-\frac{\sum_{i} f_{i}^{2}(\mathbf{r})}{\sum_{i} f_{i}(\mathbf{r})} .
$$

An example of the $x y$ projection of $\sum_{i=0}^{N} f_{i}(\mathbf{r})$ and $V(\mathbf{r})$ is shown in Fig. 4.6(a) and (b), respectively. The hemisphere of tracks chosen for this plot is taken from an SLD Monte Carlo $Z^{0} \rightarrow b \bar{b}$ event in which the jet momentum is directed from left to right in Fig. 4.6. The two peaks in $V(\mathbf{r})$ can be seen in plot $(b)$. The first peak at position $(0,0)$ is due to the primary vertex (IP), while the secondary peak is displaced to the right of the IP by about $1.5 \mathrm{~mm}$. The position of the most significant $V(\mathbf{r})$ value is taken as the secondary vertex.

A SV was required to contain two or more tracks, and to be separated from the IP by at least $1 \mathrm{~mm}$. We found 14,096 events containing a SV in only one jet, 5817 events containing 


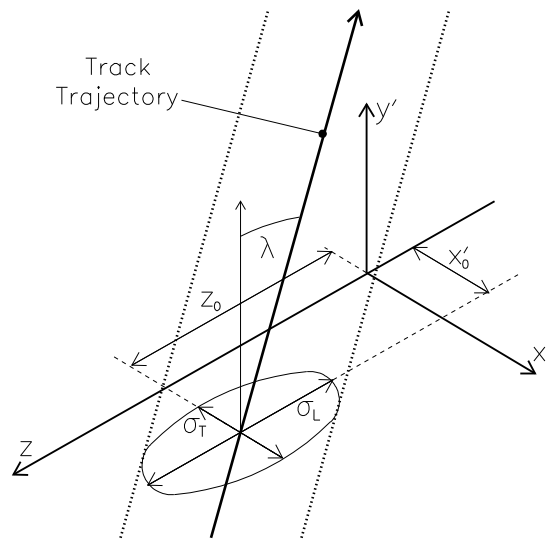

Figure 4.5: Construction of the Gaussian tube $f_{i}(\mathbf{r})$ for each track $i$ [100].
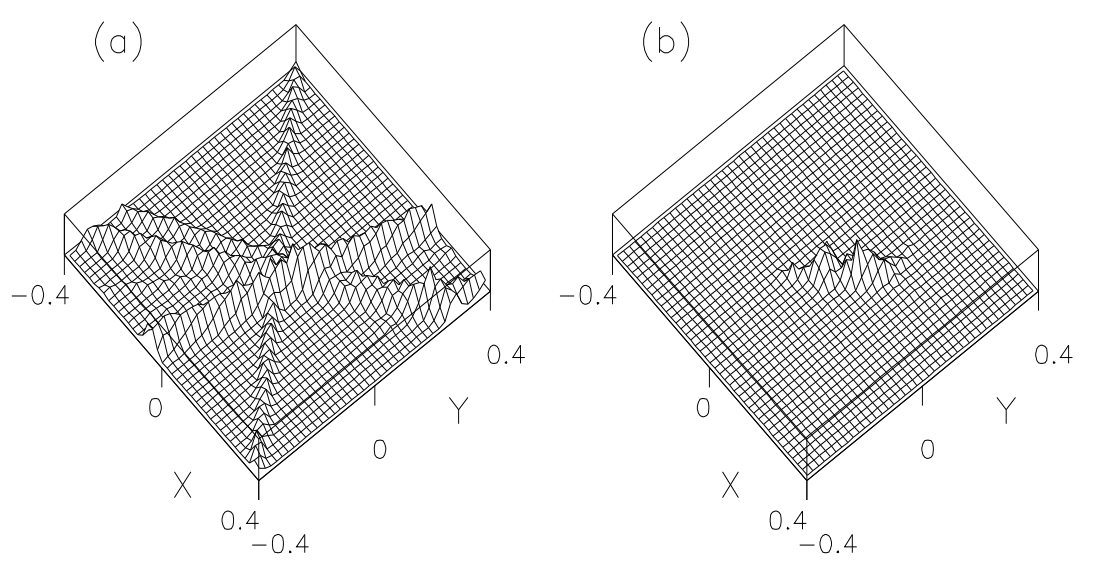

Figure 4.6: $(a)$ Track and $(b)$ vertex functions projected onto the $x y$ plane for a Monte Carlo event [100]. a SV in two jets, and 54 events containing a SV in more than two jets. The selected SVs comprise, on average, 3.0 tracks. These requirements preferentially select SVs that originate from the decay of particles with relatively long lifetime. In our simulated event sample a SV was found in $50 \%$ of all true $b$-quark hemispheres, in $15 \%$ of true $c$-quark, and in $<1 \%$ of true light-quark hemispheres [100], where hemispheres were defined by the plane normal to the thrust axis that contains the IP.

Due to the cascade structure of $B$-hadron decays, not all tracks in the decay chain will necessarily originate from a common decay point, and in such cases the SV may not be fully reconstructed in $b \bar{b}$ events. Therefore, we improved our estimate of the SV by allowing the possibility of attaching additional tracks. First, we defined the vertex axis to be the straight line joining the IP and the SV centroids, and $D$ to be the distance along this axis between the IP and the SV. For each track in the jet not included in the SV the point of closest approach (POCA), and corresponding distance of closest approach, $T$, to the vertex axis were determined. The length, $L$, of the projection of the vector joining the IP and the POCA, along the vertex axis was then calculated. Tracks with $T<1.0 \mathrm{~mm}, L>0.8 \mathrm{~mm}$, and $L / D>0.22$ were then attached to the SV. On average 0.5 tracks per SV were attached in this fashion.

The invariant mass, $M_{c h}$, of each SV was then calculated by assigning each track the charged pion mass. In order to account partially for the effect of neutral particles missing from the SV we applied a kinematic correction to the calculated $M_{c h}$. We added the momentum vectors of all tracks forming the SV to obtain the vertex momentum, $\vec{P}_{v t x}$, and evaluated the magnitude of the component of the vertex momentum transverse to the vertex axis, $P_{t}^{v}$ (Fig. 4.7). In order to reduce the effect of the IP and SV measurement errors, the vertex axis was varied within an envelope defined by all possible cotangents to the error ellipsoids of both the IP and the SV, and the minimum $P_{t}^{v}$ was chosen. We then defined the $P_{t}^{v}$-corrected vertex mass,

$$
M_{v t x}=\sqrt{M_{c h}^{2}+P_{t}^{v 2}}+\left|P_{t}^{v}\right| .
$$

The distributions of $M_{v t x}$ and $P_{v t x}$ are shown in Fig. 4.8; the data are reproduced by the simulation, in which the primary event-flavor breakdown is indicated. The region $M_{v t x}$ $>2 \mathrm{GeV} / c^{2}$ is populated predominantly by $Z^{0} \rightarrow b \bar{b}$ events, whereas the region $M_{v t x}<2$ $\mathrm{GeV} / c^{2}$ is populated roughly equally by $b \bar{b}$ and non- $b \bar{b}$ events. 


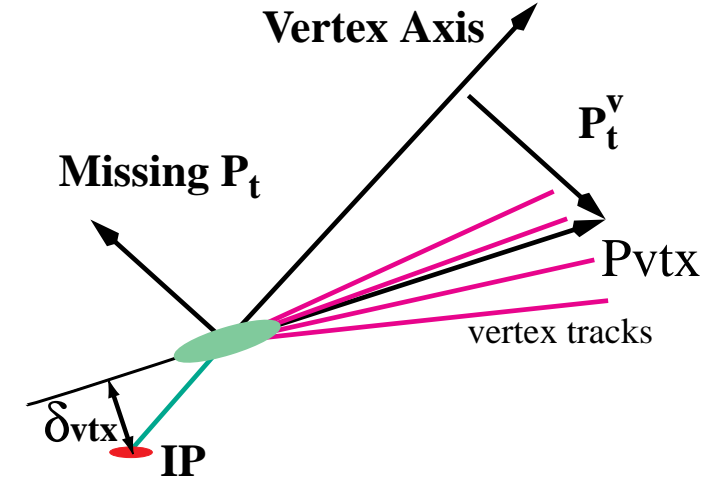

Figure 4.7: Schematic description of missing transverse momentum, $P_{t}^{v}$, and vertex impact parameter, $\delta_{v t x}$

\subsubsection{Requirements for Flavor Tags}

In order to optimize the separation among flavors we examined the two-dimensional distribution of $P_{v t x}$ vs. $M_{v t x}$. The distribution for events containing a SV is shown in Fig. 4.9 for the data and simulated samples; the data (Fig. 4.9a) are reproduced by the simulation (Fig. 4.9b). The distributions for the simulated subsamples corresponding to true primary $b \bar{b}, c \bar{c}$, and $q_{l} \overline{q_{l}}\left(q_{l}=u+d+s\right)$ events are shown in Figs. $4.9 \mathrm{c}, 4.9 \mathrm{~d}$ and $4.9 \mathrm{e}$ respectively. These figures show that vertices from charm decay typically have a higher momentum for the same mass than those from $b$ quark decays. The momentum information of SV uses the fact that $b$ fragmentation is harder than that of $c$ quarks. At the same vertex mass, the relation :

$$
M_{v t x}^{2}=E_{b}^{2}-P_{b}^{2}=E_{c}^{2}-P_{c}^{2}
$$

holds between energy, $E_{q}$, and momentum, $P_{q}$, of type $q \mathrm{SV}$. The signature of harder fragmentation of $b$ hadron implies that $E_{b}$ is larger than $E_{c}$ and $P_{b}$ is smaller than $P_{c}$.

In order to separate $b \bar{b}$ and $c \bar{c}$ events from each other, and from the $q_{l} \bar{q}_{l}$ events, we defined regions:

(A) $M_{v t x}>1.8$ and $P_{v t x}+10<15 M_{v t x}$
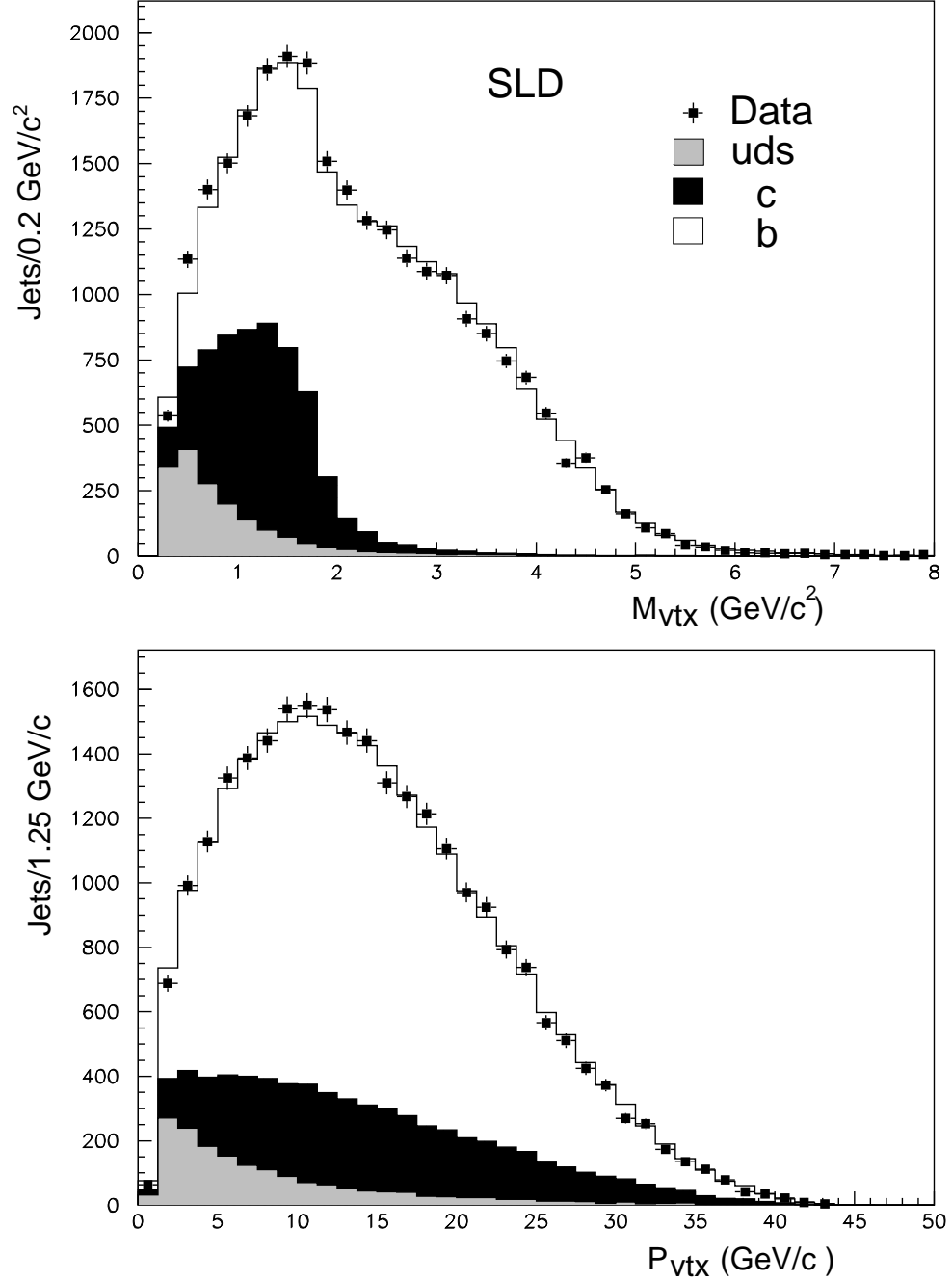

Figure 4.8: The distribution of the corrected vertex-mass $M_{v t x}$ and the vertex momentum $P_{v t x}$ in our data sample (points); the simulated distributions are shown as histograms in which the contributions from events of different primary quark flavor are indicated. 
(B) $M_{v t x}<1.8$ and $P_{v t x}>5$ and $P_{v t x}+10 \geq 15 M_{v t x}$ where $M_{v t x}\left(P_{v t x}\right)$ is in units of $\mathrm{GeV} / c^{2}$ $(\mathrm{GeV} / c)$.

(C) all remaining events containing a SV

The boundaries of regions (A) and (B) are indicated in Figs. 4.9c and 4.9d, respectively, and all three regions are labeled in Fig. 4.9f.

The $b$-tagged sample (subsample 1) was defined to comprise those events containing any vertex in region $(\mathrm{A})$.

For the remaining events containing any vertex in region $(\mathrm{B})$ we examined the distribution of the impact parameter of the vector $\overrightarrow{P_{v t x}}$ w.r.t. the IP, $\delta_{v t x}$ (Fig. 4.7); according to the simulation true primary $c \bar{c}$ events dominate the population in the region $\delta_{v t x}<0.02 \mathrm{~cm}$ (Fig. 4.10). Therefore, we defined the $c$-tagged sample (subsample 2) to comprise those events in region (B) with $\delta_{v t x}<0.02 \mathrm{~cm}$. This $\delta_{v t x}$ cut increases the charm tag purity by $3 \%$ and loses $2.5 \%$ in efficiency.

Events containing no selected SV were then examined. For such events the distribution of $N_{s i g}$, the number of tracks per event that miss the IP by $d>2 \sigma_{d}$, is shown in Fig 4.11. The $u d s$-tagged sample (subsample 3) was defined to comprise those events with $N_{\text {sig }}=0$. All events not assigned to subsample 1,2, or 3 were defined to comprise the untagged sample (subsample 4).

Using the simulation we estimated that the efficiencies $\epsilon^{j i}$ for selecting events (after acceptance cuts) of type $i(i=u d s, c, b)$ into subsample $j(j=u d s, c, b$, untagged $)$, and the fractions $\Pi^{j i}$ of events of type $i$ in subsample $j$, are :

$$
\begin{aligned}
& \varepsilon^{j i}=\left(\begin{array}{ccc}
56.4 \pm 0.1 & 18.6 \pm 0.1 & 1.65 \pm 0.04 \\
1.51 \pm 0.02 & 19.1 \pm 0.1 & 4.25 \pm 0.06 \\
0.35 \pm 0.01 & 2.48 \pm 0.05 & 61.5 \pm 0.1 \\
41.7 \pm 0.1 & 59.8 \pm 0.2 & 32.6 \pm 0.1
\end{array}\right) \\
& \Pi^{j i}=\left(\begin{array}{ccc}
90.6 \pm 0.1 & 8.4 \pm 0.1 & 0.93 \pm 0.03 \\
17.7 \pm 0.2 & 64.4 \pm 0.3 & 17.9 \pm 0.2 \\
1.50 \pm 0.04 & 3.0 \pm 0.1 & 95.5 \pm 0.1 \\
59.3 \pm 0.1 & 24.1 \pm 0.1 & 16.6 \pm 0.1
\end{array}\right)
\end{aligned}
$$

These diagonal elements are summarized in Table 4.2. The purity and efficiency for the $b$-tag subsample are $95.8 \%$ and $61.6 \%$, respectively. As for the $c$-tag subsample, the purity is substantially improved compared with the conventional lifetime based flavor tagging purity of $\sim 30 \%$, although its efficiency looks relatively small due to the tight tag criteria. As cross checks, we investigated many different flavor tagging criteria. which will be described in Section 5.6.

Table 4.2: Summary of flavor-tagged subsamples
\begin{tabular}{|cc|c|c|c|}
\hline \multicolumn{2}{|c|}{ subsample } & \# of events & purity & efficiency \\
\hline 1 & uds & 30298 & 90.6 & 56.4 \\
2 & $c$ & 4171 & 64.4 & 19.1 \\
3 & $b$ & 10772 & 95.5 & 61.5 \\
4 & untagged & 32655 & - & - \\
\hline
\end{tabular}



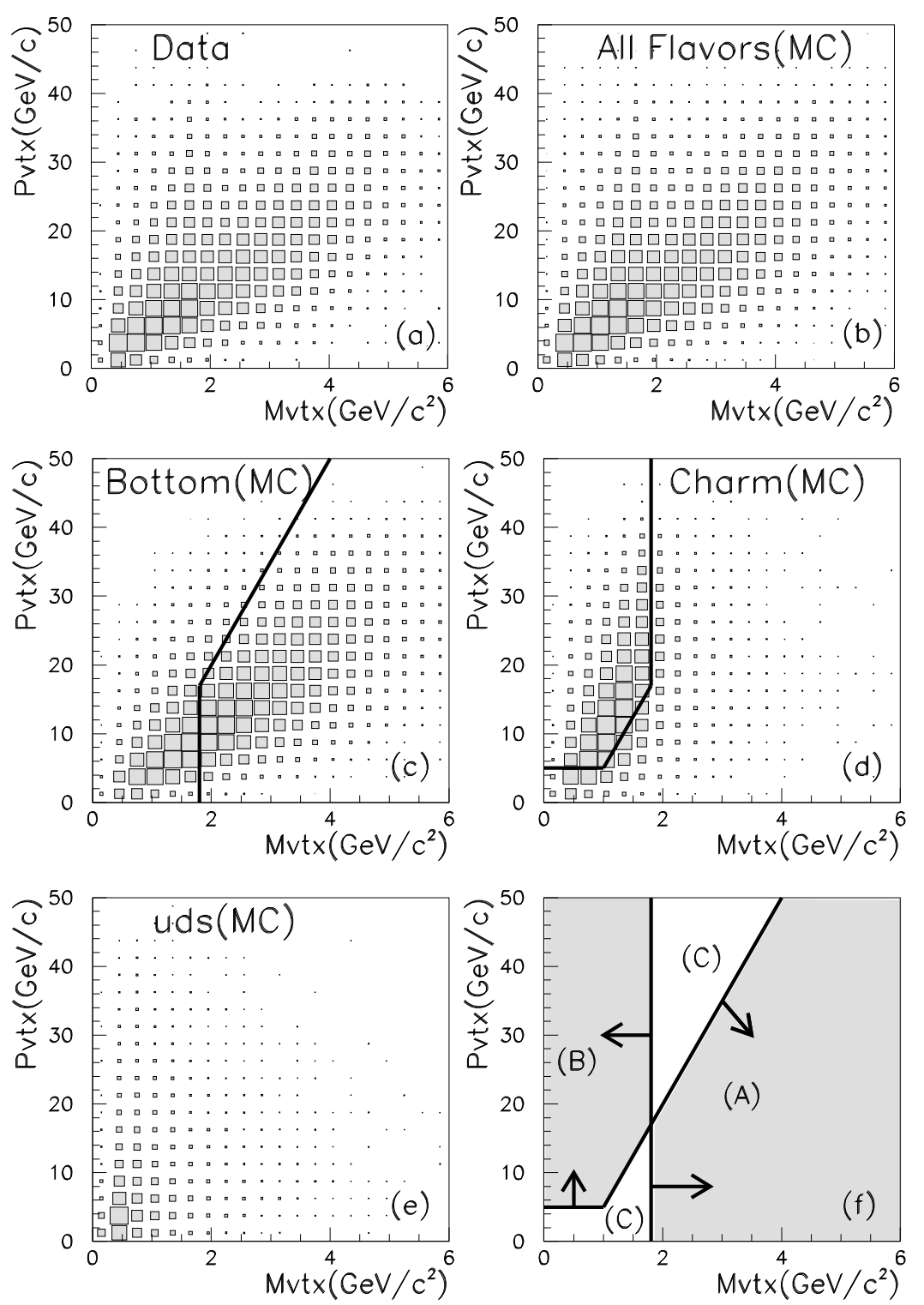

Figure 4.9: The two-dimensional distribution of vertex momentum $P_{v t x}$ vs. vertex mass $M_{v t x}$ (see text). (a) Data; (b) all-flavors simulation; (c) $b \bar{b}$ event simulation; (d) $c \bar{c}$ event simulation; (e) $q_{l} \bar{q}_{l}$ event simulation. In (f) the regions used for $b$-tagging (A), $c$-tagging (B) and no-tagging $(\mathrm{C})$ are indicated (see text).

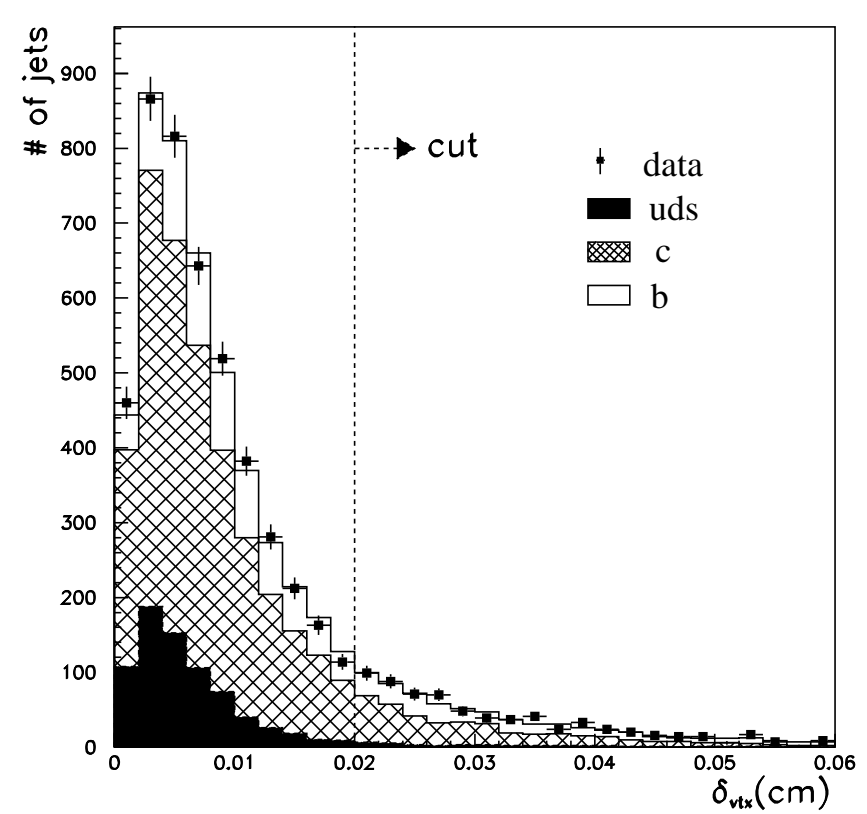

Figure 4.10: The distribution of vertex impact parameter, $\delta_{v t x}$ for events in region (B): data (points); the simulated distribution is shown as a histogram in which the contributions from events of different primary quark flavor are indicated 


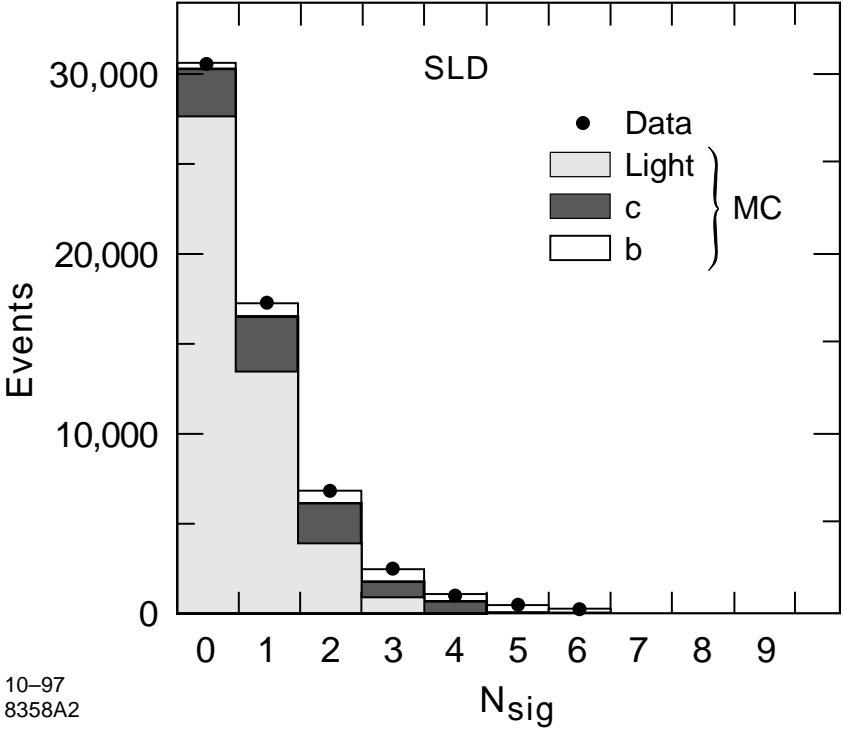

Figure 4.11: The distribution of the number of tracks per event that miss the IP by at least $2 \sigma$ in terms of their impact parameter in the plane normal to the beamline, in events that contain no reconstructed vertex (see text); data (points). The simulated distribution is shown as a histogram in which the contribution from events of different primary quark flavor are indicated.

\section{Chapter 5}

\section{Measurement of the $\alpha_{s}($ flavor $)$ ratios}

\subsection{Introduction}

Here we present the test of the flavor-independence of strong interaction in terms of the ratios $\alpha_{s}^{i} / \alpha_{s}^{\text {all }}(i=u d s, c, b)$. From the beginning of this analysis, it has been clear that the most precise test of the flavor-independence of strong interactions must come from measuring a ratio of the strong couplings of one quark flavor to another sample. As described in Sect. 2.2.1, determination of the absolute value of $\alpha_{s}$ is limited by the theoretical uncertainties predominantly due to the renormalization scale, which comes from the finite order expansions of perturbative QCD. By taking the ratio, most of the common uncertainties related to the absolute value of $\alpha_{s}$ such as detector effects, hadronization models, and renormalization scale, are largely canceled.

The ratios $\alpha_{s}^{i} / \alpha_{s}^{\text {all }}$ can be derived from the respective measured ratios $R_{3}^{i} / R_{3}^{\text {all }}$, where $R_{3}$ is the rate of the 3 -jet production to the total hadronic events :

$$
R_{3}=\frac{\sigma_{3-j e t}}{\sigma_{\text {had }}}
$$

which is directly proportional to $\alpha_{s}$ at leading order.

The translation from the $R_{3}$ ratio to the $\alpha_{s}$ ratio is subject to the theoretical calculations. The 3-jet rate in heavy-quark events is expected to be modified relative to that in light-quark events by the effect of the non-zero quark masses. This effect needs to be corrected when the $R_{3}$ ratios are translated to $\alpha_{s}$ ratios. In this thesis, we applied $\mathcal{O}\left(\alpha_{s}^{2}\right)$ calculations in order to translate the $R_{3}$ ratio to $\alpha_{s}$ ratios taking into account heavy quark mass effects. 
The measurement of $\alpha_{s}$ ratios was accomplished by six different jet clustering algorithms ( E0, E, P, P0, Durham, Geneva ). These results were then compared to evaluate systematic uncertainties.

\subsection{Jet Finding}

\subsubsection{Jet Clustering Algorithms}

A useful method of classifying the structure of hadronic final states is in terms of jets. Jets are reconstructed using iterative clustering algorithms [70] in which a measure $y_{i j}$, such as scaled invariant mass, is calculated for all pairs of particles $i$ and $j$, and the pair with the smallest $y_{i j}$ is combined into a single (pseudo-)particle. This procedure is repeated until all pairs have $y_{i j}$ exceeding a value $y_{c u t}$, and the jet multiplicity of the event is defined as the number of particles remaining. The $n$-jet rate $R_{n}\left(y_{c u t}\right)$ is the fraction of events classified as $n$-jet.

Several algorithms have been proposed featuring different $y_{i j}$ definitions and recombination methods. In this analysis, we have applied the E, E0, P, and P0 variations of the JADE algorithm [68] as well as the Durham (D) and Geneva (G) schemes [70]. All of these algorithms are collinear- and infrared-safe, thus the jet rate can be calculated in perturbative QCD. The six definitions of the jet resolution parameter $y_{i j}$ and recombination procedure are given below.

In the E-scheme, for instance, $y_{i j}$ is defined as the square of the invariant mass of the pair of particles $i$ and $j$ scaled by the visible energy in the event :

$$
y_{i j}=\frac{\left(p_{i}+p_{j}\right)^{2}}{E_{v i s}^{2}}
$$

with the recombination performed as

$$
p_{k}=p_{i}+p_{j}
$$

where $p_{i}$ and $p_{j}$ are four-momenta of the particles and pion masses are assumed in calculating particle energies. Energy and momentum are explicitly conserved in this scheme.

The E0-, P-, and P0-schemes are variations of the E-scheme. In the E0-scheme $y_{i j}$ is defined by Eq. 5.2, while the recombination procedure is defined by

$$
\begin{aligned}
E_{k} & =E_{i}+E_{j} \\
\vec{p}_{k} & =\frac{E_{k}}{\left|\vec{p}_{i}+\vec{p}_{j}\right|}\left(\vec{p}_{i}+\vec{p}_{j}\right)
\end{aligned}
$$

where $E_{i}$ and $E_{j}$ are the energies, and $\vec{p}_{i}$ and $\vec{p}_{j}$ are the three-momenta of the particles. The three-momentum $\vec{p}_{k}$ is rescaled so that particle $k$ has zero invariant mass. This scheme does not conserve the total momentum sum of an event.

In the P-scheme $y_{i j}$ is defined by Eq. 5.3 and the recombination procedure is defined by

$$
\begin{aligned}
\vec{p}_{k} & =\vec{p}_{i}+\vec{p}_{j} \\
E_{k} & =\left|\vec{p}_{k}\right| .
\end{aligned}
$$

This scheme conserves the total momentum of an event, but does not conserve the total energy.

The P0-scheme is similar to the P-scheme, but the total energy $E_{v i s}$ in Eq. 5.2 is recalculated at each iteration according to

$$
E_{v i s}=\sum_{k} E_{k} .
$$

In the D-scheme $y_{i j}$ is definded by

$$
y_{i j}=\frac{2 \min \left(E_{i}^{2}, E_{j}^{2}\right)\left(1-\cos \theta_{i j}\right)}{E_{v i s}^{2}}
$$

where $\theta_{i j}$ is the angle between the pair of particles $i$ and $j$. The recombination is defined by Eq. 5.3. With the D-scheme, a soft particle will only be combined with another soft particle, instead of being combined with a high-energy particle, if the angle it makes with the other soft particle is smaller than the angle that makes with the high-energy particle.

The definition of $y_{i j}$ for the G-scheme is

$$
y_{i j}=\frac{8 E_{i} E_{j}\left(1-\cos \theta_{i j}\right)}{9\left(E_{i}+E_{j}\right)^{2}}
$$

and the recombination is defined by Eq. 5.3. Note that $y_{i j}$ depends only on the energy of the particles to be combined, and not on the $E_{v i s}$ of the event. In this scheme soft particles are combined as in the D-scheme. 
Definitions of $y_{i j}$ and recombination schemes are summarized in Table. 5.1. Each jet algorithm is applied to the "well-measured tracks" described in Sect. 4.1.3 to define "detectorlevel" jets. For the Monte Carlo sample, jet algorithms are applied to the particles at the end of Parton Shower evolution, to all stable particles, as well as at the detector-level. We define these as "parton-level" and "hadron-level" jets, respectively.

\begin{tabular}{|c|c|c|}
\hline scheme & resolution parameter & recombination \\
\hline $\mathrm{E}$ & \multirow{4}{*}{$\left(p_{i}+p_{j}\right)^{2} / Q^{2}$} & $\begin{array}{c}p_{k}=p_{i}+p_{j} \\
Q=E_{v i s}\end{array}$ \\
\hline E0 & & $\begin{array}{c}E_{k}=E_{i}+E_{j} \\
\vec{p}_{k}=\frac{E_{k}}{\left|\vec{p}_{i}+\vec{p}_{j}\right|}\left(\vec{p}_{i}+\vec{p}_{j}\right) \\
Q=E_{v i s}\end{array}$ \\
\hline $\mathrm{P}$ & & $\begin{array}{c}\vec{p}_{k}=\vec{p}_{i}+\vec{p}_{j} \\
E_{k}=\left|\vec{p}_{k}\right| \\
Q=E_{v i s}\end{array}$ \\
\hline P0 & & $\begin{array}{c}\vec{p}_{k}=\vec{p}_{i}+\vec{p}_{j} \\
E_{k}=\left|\vec{p}_{k}\right| \\
Q=\sum_{k} E_{k}\end{array}$ \\
\hline $\mathrm{D}$ & $\frac{2 \min \left(E_{i}^{2}, E_{j}^{2}\right)\left(1-\cos \theta_{i j}\right)}{E_{v i s}^{2}}$ & $p_{k}=p_{i}+p_{j}$ \\
\hline G & $\frac{8 E_{i} E_{j}\left(1-\cos \theta_{i j}\right)}{9\left(E_{i}+E_{j}\right)^{2}}$ & $p_{k}=p_{i}+p_{j}$ \\
\hline
\end{tabular}

Table 5.1: Definition of the jet resolution parameter $y_{i j}$ and of recombination schemes for the jet clustering algorithm.

As an example, the $y_{c u t}$ dependence of the n-jet rate is illustrated in Fig. 5.1 for the $E 0$ algorithm. The $y_{c u t}$ represents the jet resolution scale parameter. Large $y_{\text {cut }}$ values corresponds to poor eyesight, and tend to classify most of the events into 2-jet-like. On the other hand, small $y_{c u t}$ correspond to good eyesight, and a richer event structure can be seen.

The $y_{c u t}$ values for each jet algorithm were chosen to minimize the total uncertainties. They are listed in Table 5.2, and also will be discussed later with the error estimation in Sect. 5.5.4.
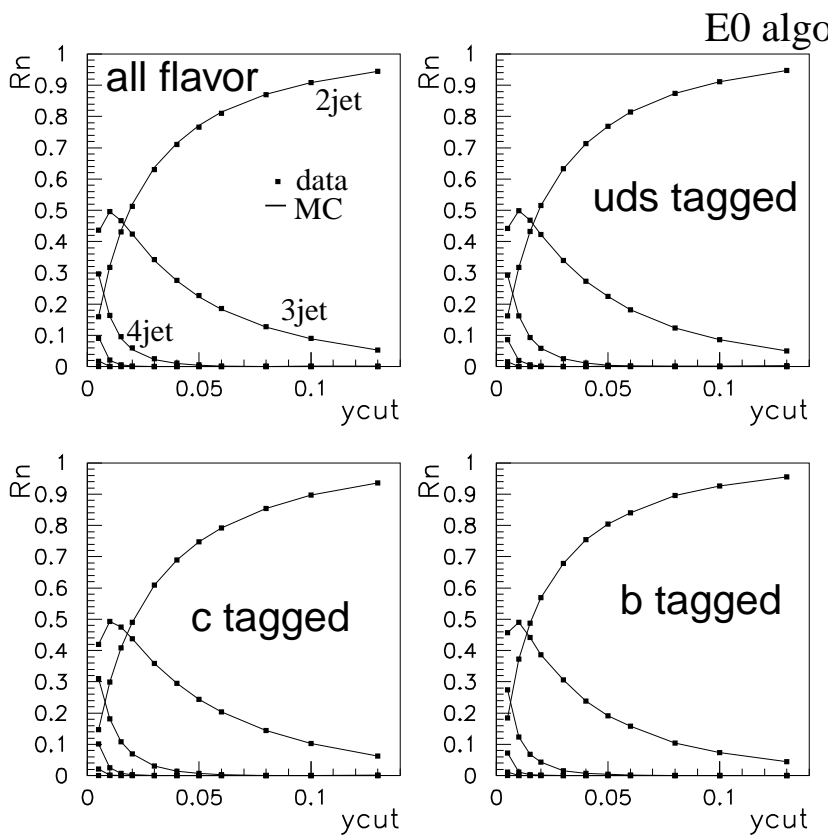

Figure 5.1: The detector-level n-jet rate vs. $y_{\text {cut }}$. The points are Data and lines are MC.

\section{$5.3 \quad R_{3}^{\text {flavor }} / R_{3}^{\text {all }}$ derivation}

\subsubsection{Detector level jet rate}

Each of the six jet-finding algorithms was applied to each event subsample $j, 1 \leq j \leq 4$, as well as to the global sample of all accepted events ('all'). In each case events were divided into two categories: (i) two jets, and (ii) three or more jets. The number of 2 - and $\geq 3$-jet 
events $\left(n_{2}{ }^{j}\right.$ and $n_{3}{ }^{j}$, respectively) for each tagged flavor and each algorithm are summarized in Table. 5.2 at the chosen $y_{\text {cut }}$ points. The fraction of the event sample in category (ii) was defined as the detector level 3-jet rate $R_{3}^{j}=n_{3}^{j} /\left(n_{2}^{j}+n_{3}^{j}\right)$ for each subsumple $j$. The measured 3-jet rate $R_{3}^{j}$ are shown as a function of $y_{\text {cut }}$ for each subsample and each algorithm in Fig. 5.2. The ratios $R_{3}^{j} / R_{3}^{\text {all }}$ for each subsample are shown in the upper figures in Fig. 5.5(a)-5.9(a). The results of the corresponding analysis applied to the simulated event sample are also shown; the simulation reproduces the data.

Table 5.2: The number of 2- and $\geq 3$-jet events for each subsample and algorithm.

\begin{tabular}{|c||c|c|c|c|c|c|c|c|c|}
\hline Jet Algorithm & $y_{\text {cut }}$ & $n_{2}{ }^{u d s}$ & $n_{2}{ }^{c}$ & $n_{2}{ }^{b}$ & $n_{2}{ }^{n o-t a g}$ & $n_{3}{ }^{u d s}$ & $n_{3}{ }^{c}$ & $n_{3}{ }^{b}$ & $n_{3}{ }^{n o-t a g}$ \\
\hline E & 0.040 & 16387 & 2257 & 5931 & 15853 & 13911 & 1914 & 4841 & 16802 \\
\hline E0 & 0.020 & 16097 & 2125 & 5952 & 15673 & 14201 & 2046 & 4820 & 16982 \\
\hline P & 0.020 & 18395 & 2507 & 6711 & 18174 & 11903 & 1664 & 4061 & 14481 \\
\hline D & 0.015 & 15272 & 2068 & 5671 & 14774 & 15026 & 2103 & 5101 & 17881 \\
\hline G & 0.010 & 21372 & 3011 & 7718 & 21164 & 8926 & 1160 & 3054 & 11491 \\
\hline
\end{tabular}

\section{Flavor Tag Bias}

We pause briefly in the description of the analysis to discuss the flavor-tagging bias. The flavor-tagging bias effect is evident in the figures in Fig. 5.5(a)-5.9(a), where it can be seen that the measured values of $R_{3}^{j} / R_{3}^{\text {all }}$ are slightly below unity for subsamples $j=1,2$, and 3 , implying that the flavor tags preferentially select 2 -jet rather than 3 -jet events. It should be noted that, as a corollary, the untagged event sample, subsample 4, contains an excess of 3 -jet events.

We define the normalized difference in efficiencies for correctly tagging a 2-jet event and a 3 -jet event of type $i(i=u d s, c$ and $b)$ in subsample $j(j=1,2$ and 3$)$ as the tag bias:

$$
\mathcal{B}^{j i}=\frac{\epsilon_{2 j e t}^{j i}-\epsilon_{3 j e t}^{j i}}{\epsilon_{2 j e t}^{j i}}
$$

For example, $\mathcal{B}^{1, b}, \mathcal{B}^{2, c}$, and $\mathcal{B}^{3, u d s}$ are $5.7 \%, 14.5 \%$, and $4.1 \%$, respectively in E0 scheme at $y_{\text {cut }}=0.02$. These biases are considerably smaller than those found in [9], which resulted from the kinematic signatures employed for flavor-tagging. Fig 5.10 shows that $\mathcal{B}^{1, u d s}, \mathcal{B}^{2, c}$ and $\mathcal{B}^{3, b}$ are flat against $y_{c u t}$.

The cause of the flavor-tag bias in our technique arises as follows: Quarks in $q \bar{q} g$ events have smaller available momentum than those in $q \bar{q}$ events. As a result, fragmented hadrons in $q \bar{q} g$ event are less energetic than those in $q \bar{q}$ event. The detector has momentum dependences on the resolution to reconstruct tracks discussed in Sect.3.4. For lower momentum tracks, extrapolation uncertainty to IP becomes large, significance from IP is diminished, and trajectories of tracks will be less precise around IP for those hadrons. Consequently, in the heavy quark $(Q)$ events, the secondary vertices (SV) in $Q \bar{Q} g$ events will be less likely to be well-reconstructed than those in $Q \bar{Q}$ events, and the contamination from heavy flavor in the $N_{s i g}=0$ bin for $u d s$ tagging increases in 3-jet events.

\subsubsection{Parton level $R_{3}$ Unfolding}

For each jet algorithm and $y_{c u t}$ value the parton-level 3-jet rate $R_{3}^{i}$ for each of the $i$ quark types $(i=u d s, c, b)$ was extracted from a simultaneous maximum likelihood fit to $n_{2}^{j}$ and $n_{3}^{j}$, the number of 2 -jet and 3 -jet events, respectively, in the flavor-tagged subsample $(1 \leq j \leq 3)$ using the relations:

$$
\begin{aligned}
& n_{2}^{j}=\sum_{i=1}^{3}\left(\varepsilon_{(2 \rightarrow 2)}^{j i}\left(1-R_{3}^{i}\right)+\varepsilon_{(3 \rightarrow 2)}^{j i} R_{3}^{i}\right) f_{i} N \\
& n_{3}^{j}=\sum_{i=1}^{3}\left(\varepsilon_{(3 \rightarrow 3)}^{j i} R_{3}^{i}+\varepsilon_{(2 \rightarrow 3)}^{j i}\left(1-R_{3}^{i}\right)\right) f_{i} N .
\end{aligned}
$$

Here $N$ is the total number of selected events corrected for the event selection efficiency, and $f_{i}$ is the Standard Model fractional hadronic width for $Z^{0}$ decays to quark type $i$, i.e.,

$$
f_{i}=\frac{B R\left(Z^{0} \rightarrow q_{i} \bar{q}_{i}\right)}{B R\left(Z^{0} \rightarrow \text { hadrons }\right)} .
$$

The $y_{c u t}$-dependent $3 \times 3$ matrices $\varepsilon_{(2 \rightarrow 2)}^{j i}$ and $\varepsilon_{(3 \rightarrow 3)}^{j i}$ are the efficiencies for an event of type $i$, with 2- or 3 -jets at the parton level, to pass all cuts and enter the flavor tagged subsamples $j$ as a 2- or 3-jet event, respectively. These matrices were calculated from the Monte Carlo simulation, and the systematic errors on the values of the matrix elements are discussed in Section 5.4. Mathematical details of the unfolding procedure are described in Appendix B. 
This formalism explicitly accounts for modifications of the parton-level 3-jet rate due to hadronization, detector effects, and flavor-tagging bias. The unfolded parton-level $R_{3}^{i}$ are shown in Fig. 5.3 along with the MC prediction.

\subsection{3 $R_{3}^{i} / R_{3}^{\text {all }}$ and Propagation of Errors}

As described in the previous section, we have measured the set of rates $\left\{R_{3}^{i}\right\}(i=u d s, c, b)$ for production of 3 or more hadronic jets in light-, charm- and bottom-quark events. In order to reduce both experimental and theoretical systematic effects the ratios $R_{3}^{i} / R_{3}^{\text {all }}$ were then calculated, where $R_{3}^{a l l}=\sum f_{i} R_{3}^{i}$ and $f_{i}$ is the Standard Model branching fraction for $Z^{0} \rightarrow q_{i} \overline{q_{i}}$. For each of the six jet algorithms $R_{3}^{i} / R_{3}^{\text {all }}$ are shown as a function of $y_{c u t}$ in Fig. 5.5(b)-5.9(b).

Statistical errors on those ratios were calculated in following manner [107]. In general, the propagation of the error matrix from the 'old' variable set $\vec{x}$ to 'new' variable set $\vec{y}$ is given by [108]:

$$
V(\vec{y})=S V(\vec{x}) S^{T}
$$

where $\mathrm{S}$ is the matrix whose elements are the partial derivatives:

$$
S_{i j}=\frac{\partial y_{i}}{\partial x_{j}}
$$

The error on the propagated variable $y_{i}$ is $\sigma_{i}=\sqrt{V_{i i}(\vec{y})}$, and the correlation between $y_{i}$ and $y_{j}$ is $\rho_{i j}(\vec{y})=V_{i j}(\vec{y}) /\left(\sigma_{i} \sigma_{j}\right)$.

In the case of the translation between the variable sets $R_{3}^{i}$ and $R_{3}^{i} / R_{3}^{\text {all }}$,

$$
\begin{aligned}
S_{i j} & =-\frac{f_{j} R_{3}^{i}}{R_{3}^{\text {all }}} \quad(i \neq j) \\
S_{i i} & =\frac{R_{3}^{\text {all }}-f_{i} R_{3}^{i}}{R_{3}^{\text {all }}} \quad(i=j)
\end{aligned}
$$

For example, for the E0 algorithm and $y_{c u t}=0.02$, the statistical error matrix for $R_{3}^{i}$ :

$$
V\left(R_{3}^{i}\right)=\left(\begin{array}{rrr}
0.0000424 & -0.0000567 & 0.0000024 \\
-0.0000567 & 0.0005120 & -0.0000373 \\
0.0000024 & -0.0000373 & 0.0000777
\end{array}\right)
$$

is propagated to the statistical error matrix for $R_{3}^{i} / R_{3}^{\text {all }}$ :

$$
V\left(R_{3}^{i} / R_{3}^{\text {all }}\right)=\left(\begin{array}{rrr}
0.0000880 & -0.0002920 & -0.0000170 \\
-0.0002920 & 0.0013182 & -0.0002221 \\
-0.0000170 & -0.0002221 & 0.0002255
\end{array}\right) .
$$

The values of $R_{3}^{i} / R_{3}^{\text {all }}$ at the chosen $y_{\text {cut }}$ points which will be described in Sect.5.4.5 are summarized in Table 5.3. The experimental systematic errors and hadronization uncertainties on these ratios are discussed in later (Sect. 5.4).

Table 5.3: Results for $R_{3}^{j} / R_{3}^{\text {all }}$, derived from Eq. 5.11; The errors shown are statistical.

\begin{tabular}{ccccc}
\hline Algorithm & $y_{\text {cut }}$ & $R_{3}^{\text {uds }} / R_{3}^{\text {all }}$ & $R_{3}^{c} / R_{3}^{\text {all }}$ & $R_{3}^{b} / R_{3}^{\text {all }}$ \\
\hline E & 0.040 & $0.982 \pm 0.014$ & $1.025 \pm 0.050$ & $1.031 \pm 0.022$ \\
E0 & 0.020 & $0.978 \pm 0.010$ & $1.042 \pm 0.036$ & $1.030 \pm 0.016$ \\
P & 0.020 & $0.989 \pm 0.010$ & $0.993 \pm 0.037$ & $1.036 \pm 0.017$ \\
P0 & 0.015 & $0.979 \pm 0.009$ & $1.036 \pm 0.032$ & $1.032 \pm 0.014$ \\
D & 0.010 & $1.001 \pm 0.013$ & $1.039 \pm 0.050$ & $0.965 \pm 0.021$ \\
G & 0.080 & $0.994 \pm 0.018$ & $1.034 \pm 0.069$ & $0.989 \pm 0.028$ \\
\hline
\end{tabular}


CHAPTER 5. MEASUREMENT OF THE $\alpha_{S}($ FLAVOR) RATIOS
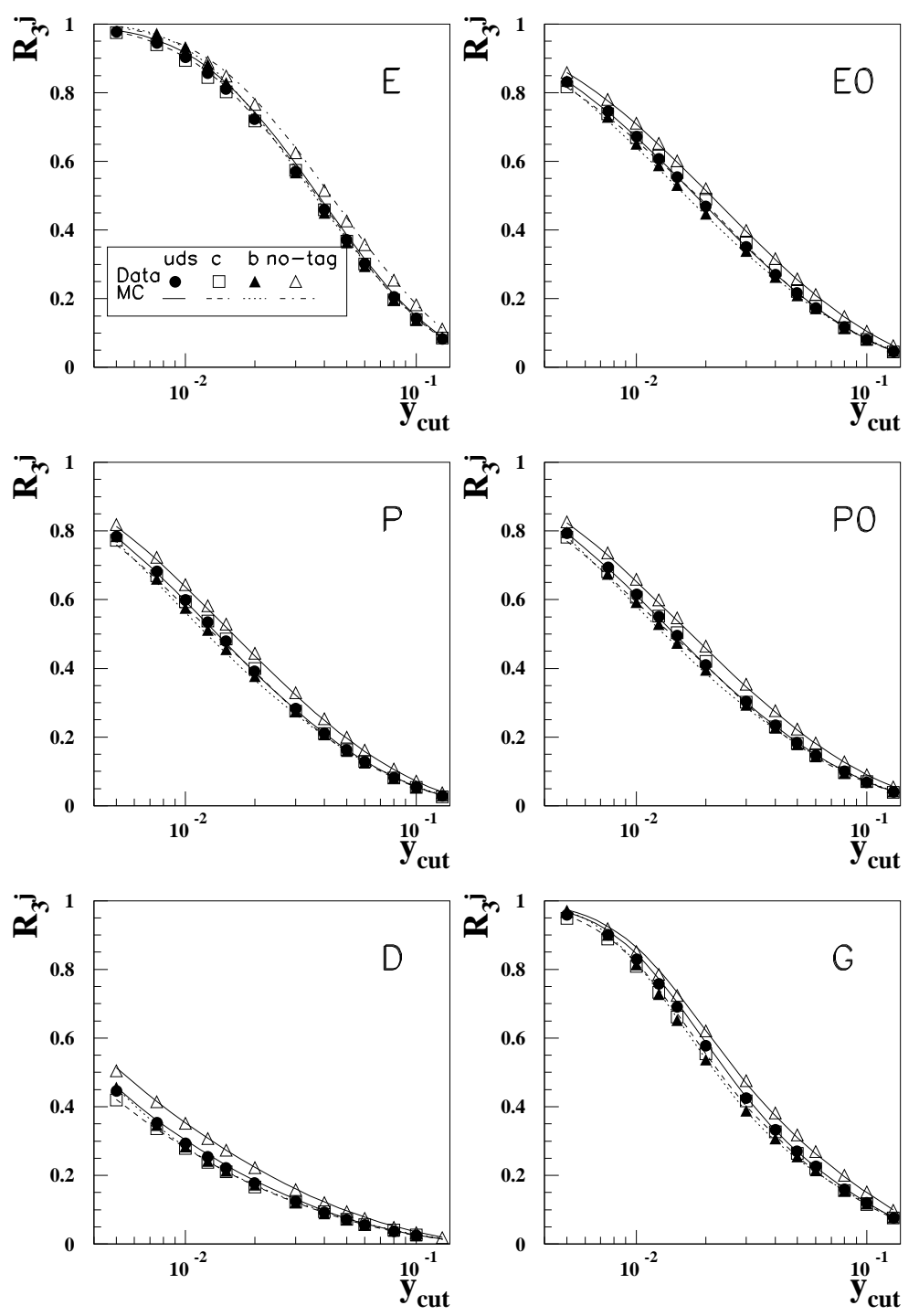

Figure 5.2: The detector-level $R_{3}^{j}$ vs. $y_{c u t}$ for each flavor-tagged subsample and for each algorithm. The points are Data and lines are MC.
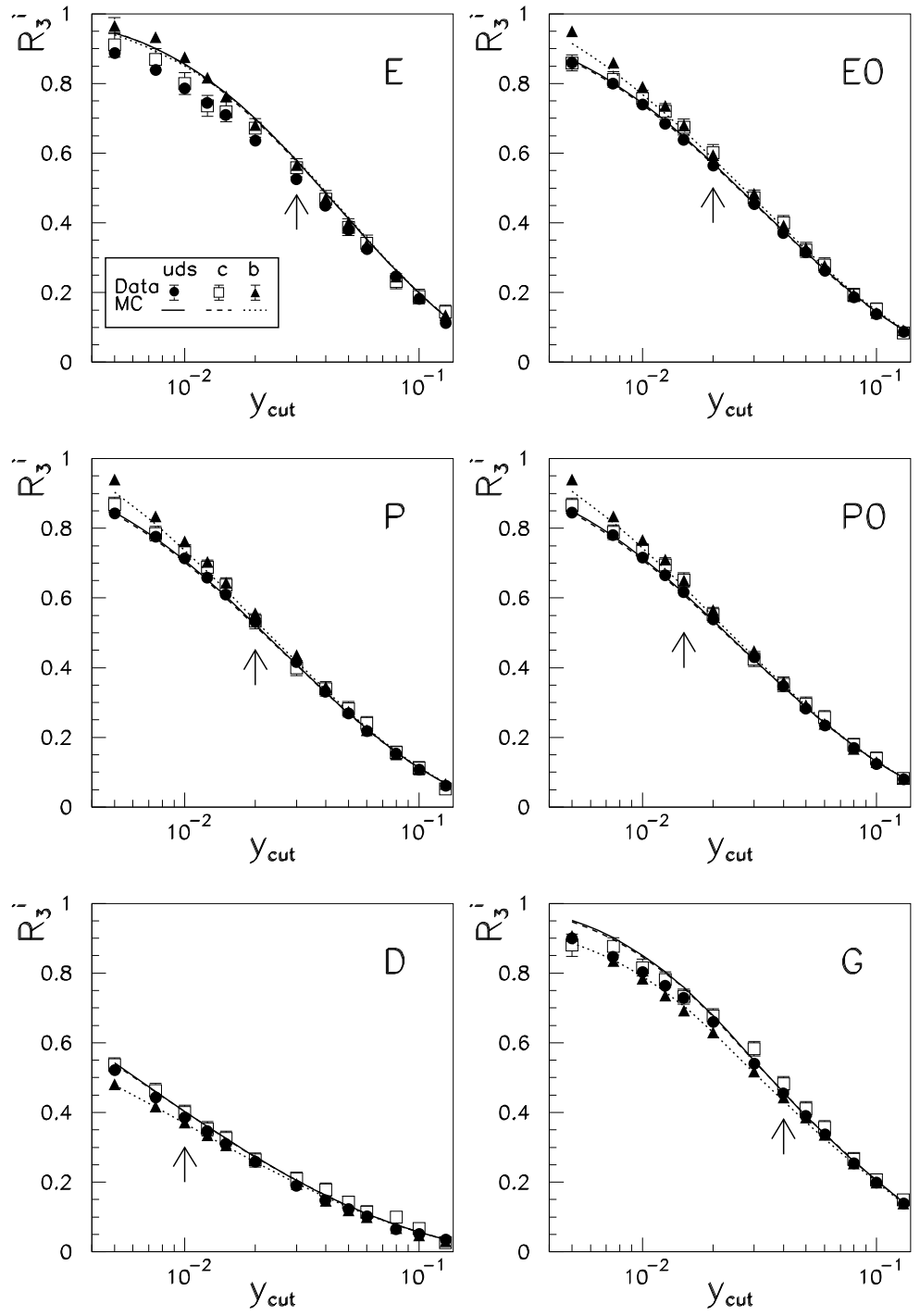

Figure 5.3: The unfolded parton-level $R_{3}$ for each flavor and different algorithms. The points are Data and lines are MC. Arrows indicate the chosen $y_{c u t}$ values. 

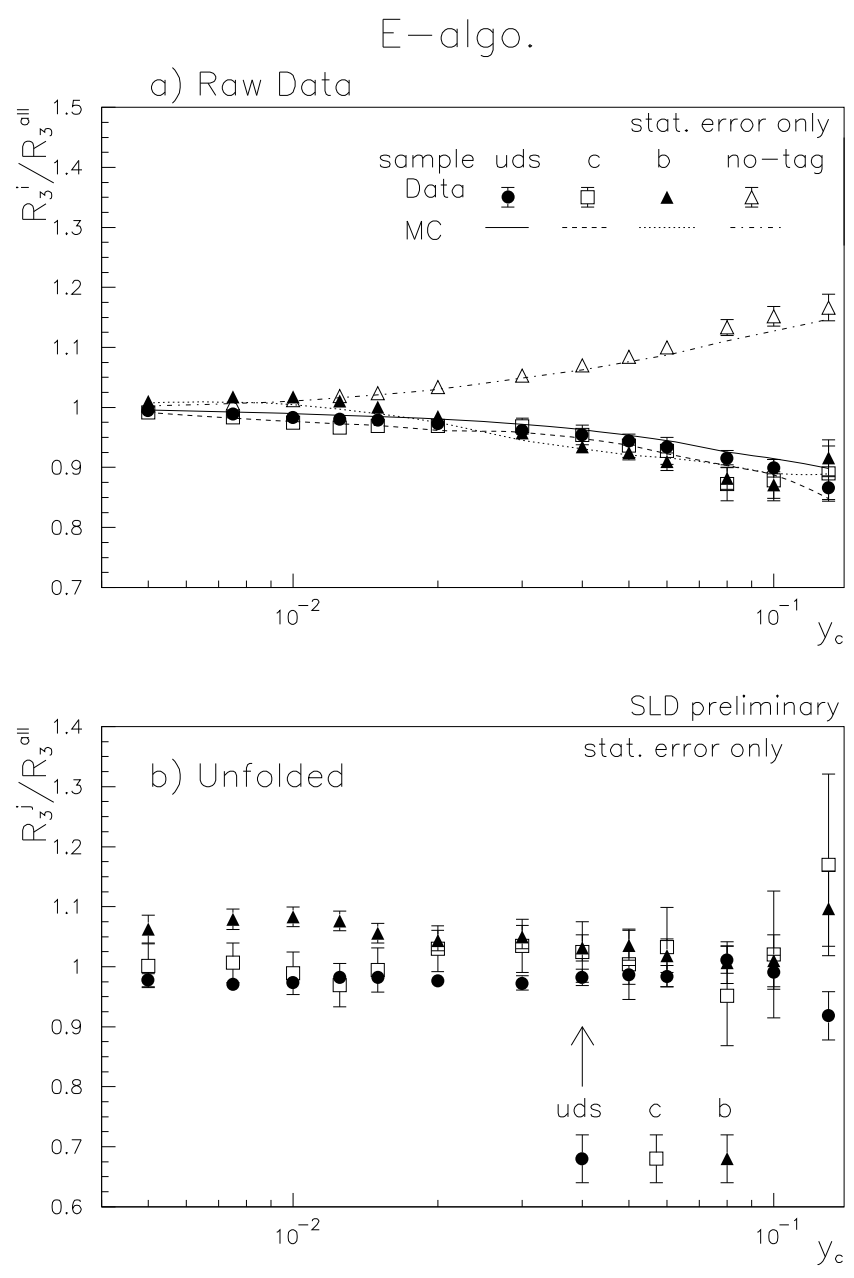

Figure 5.4: (a) The raw measured ratios $R_{3}^{j} / R_{3}^{\text {all }}$ vs. $y_{\text {cut }}$ for subsample $j$ (see text); data (points with error bars), and simulation (lines joining values at the same $y_{\text {cut }}$ values as the data). (b) The unfolded ratios $R_{3}^{i} / R_{3}^{a l l}, i=u d s, c, b$, vs. $y_{c}$ for the 3 primary event flavors. The arrow indicates the chosen $y_{c u t}$ value. : E-algorithm
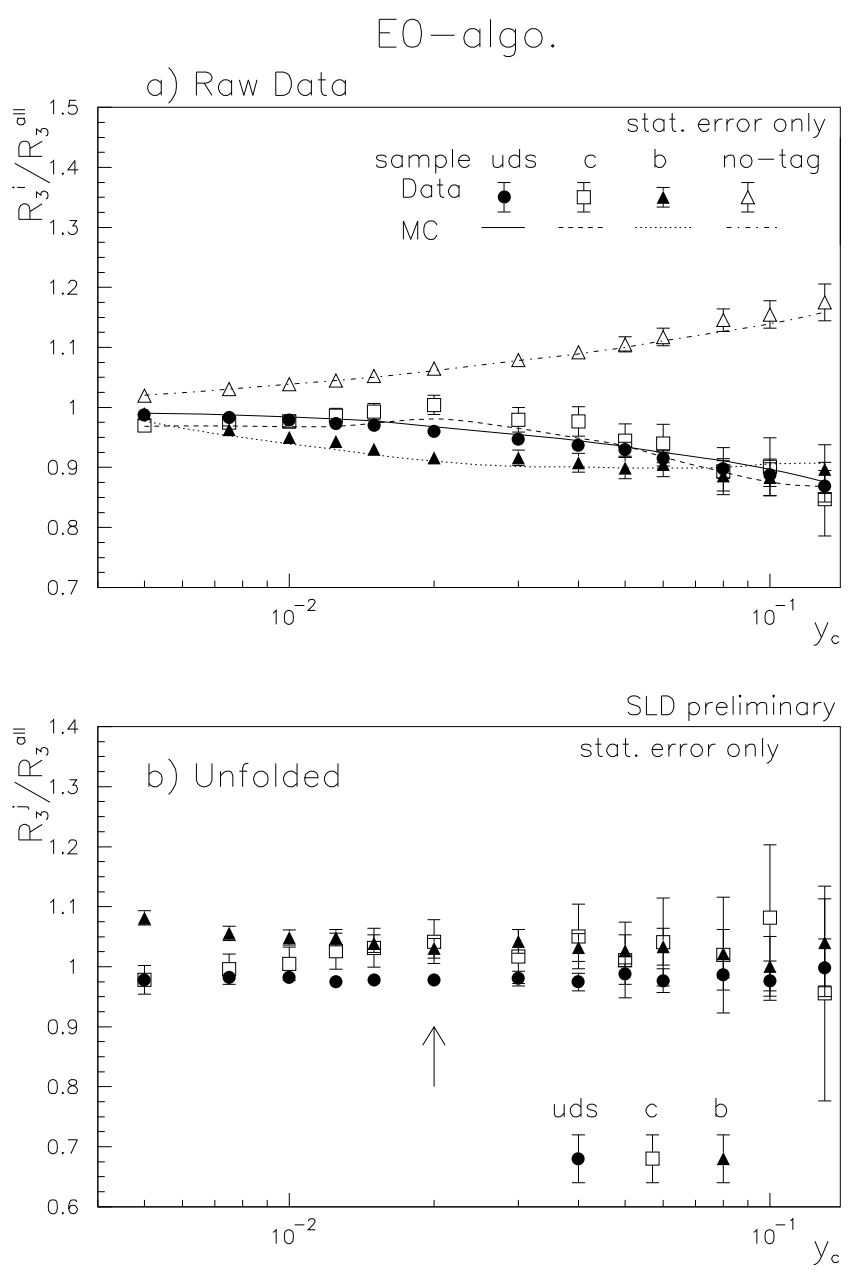

Figure 5.5: (a) The raw measured ratios $R_{3}^{j} / R_{3}^{\text {all }}$ vs. $y_{\text {cut }}$ for subsample $j$ (see text); data (points with error bars), and simulation (lines joining values at the same $y_{\text {cut }}$ values as the data). (b) The unfolded ratios $R_{3}^{i} / R_{3}^{\text {all }}, i=u d s, c, b$, vs. $y_{c}$ for the 3 primary event flavors. The arrow indicates the chosen $y_{c u t}$ value. : E0-algorithm 

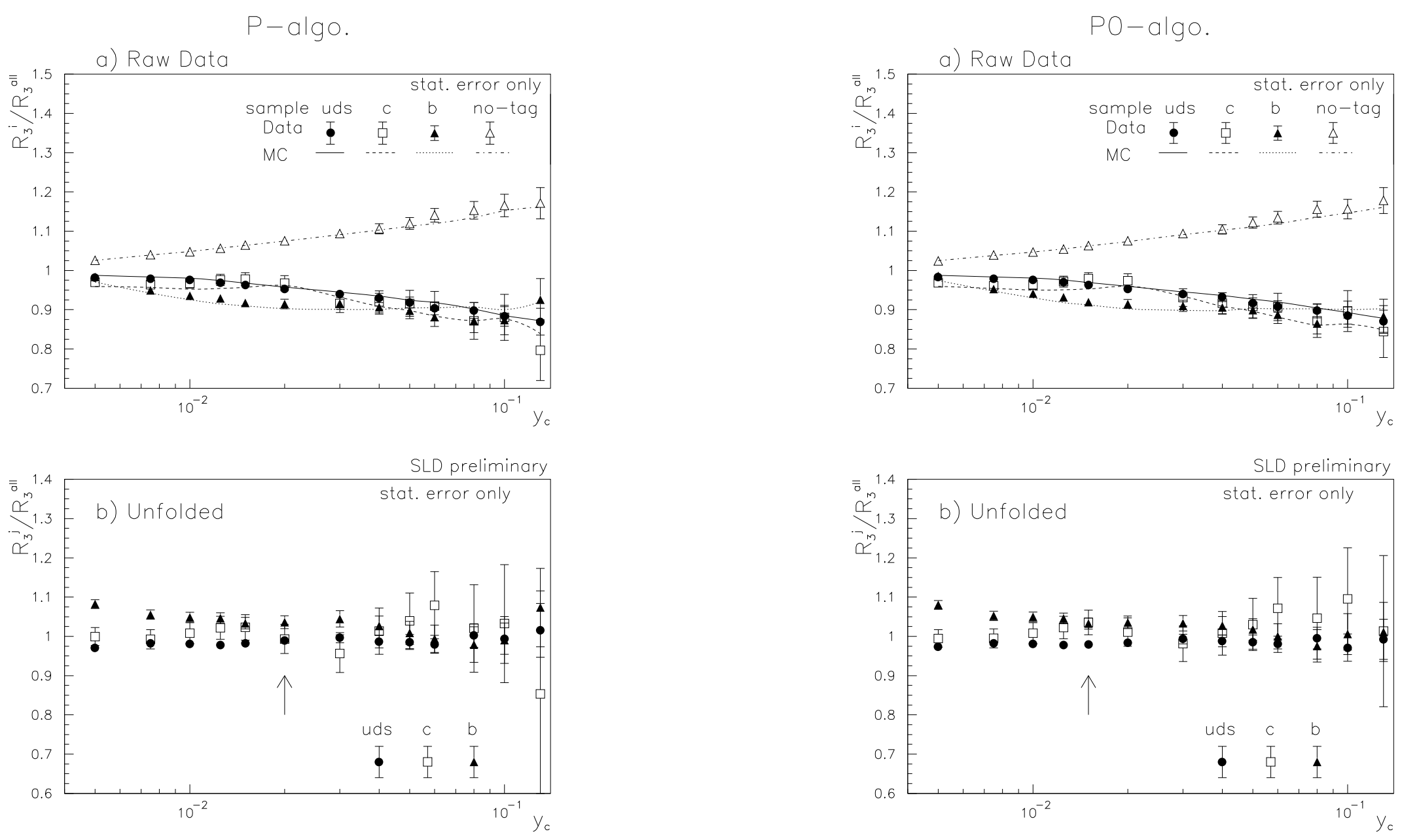

Figure 5.6: (a) The raw measured ratios $R_{3}^{j} / R_{3}^{\text {all }}$ vs. $y_{\text {cut }}$ for subsample $j$ (see text); data (points with error bars), and simulation (lines joining values at the same $y_{\text {cut }}$ values as the data). (b) The unfolded ratios $R_{3}^{i} / R_{3}^{\text {all }}, i=u d s, c, b$, vs. $y_{c}$ for the 3 primary event flavors. The arrow indicates the chosen $y_{c u t}$ value. : P-algorithm

Figure 5.7: (a) The raw measured ratios $R_{3}^{j} / R_{3}^{\text {all }}$ vs. $y_{\text {cut }}$ for subsample $j$ (see text); data (points with error bars), and simulation (lines joining values at the same $y_{\text {cut }}$ values as the data). (b) The unfolded ratios $R_{3}^{i} / R_{3}^{\text {all }}, i=u d s, c, b$, vs. $y_{c}$ for the 3 primary event flavors. The arrow indicates the chosen $y_{c u t}$ value. : P0-algorithm 
Durham
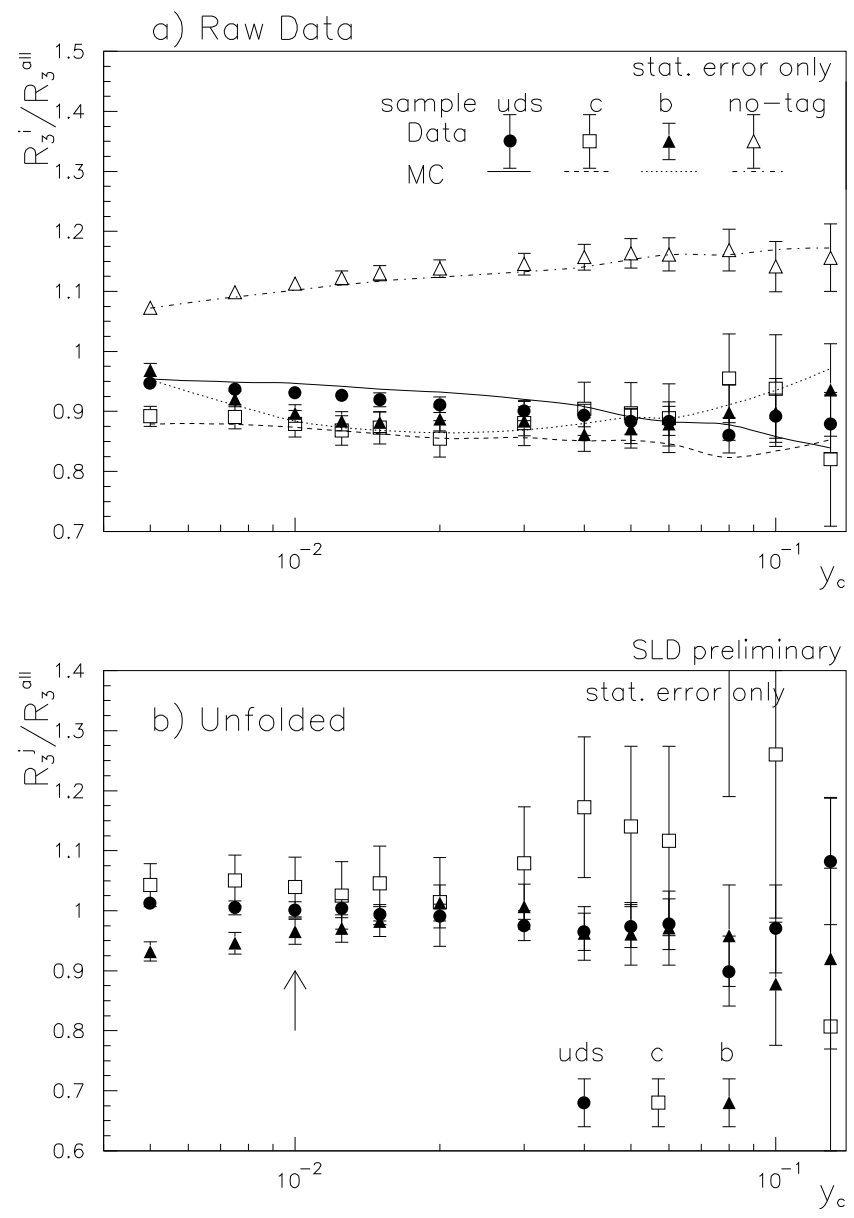

Figure 5.8: (a) The raw measured ratios $R_{3}^{j} / R_{3}^{\text {all }}$ vs. $y_{\text {cut }}$ for subsample $j$ (see text); data (points with error bars), and simulation (lines joining values at the same $y_{\text {cut }}$ values as the data). (b) The unfolded ratios $R_{3}^{i} / R_{3}^{a l l}, i=u d s, c, b$, vs. $y_{c}$ for the 3 primary event flavors. The arrow indicates the chosen $y_{c u t}$ value. : Durham-algorithm
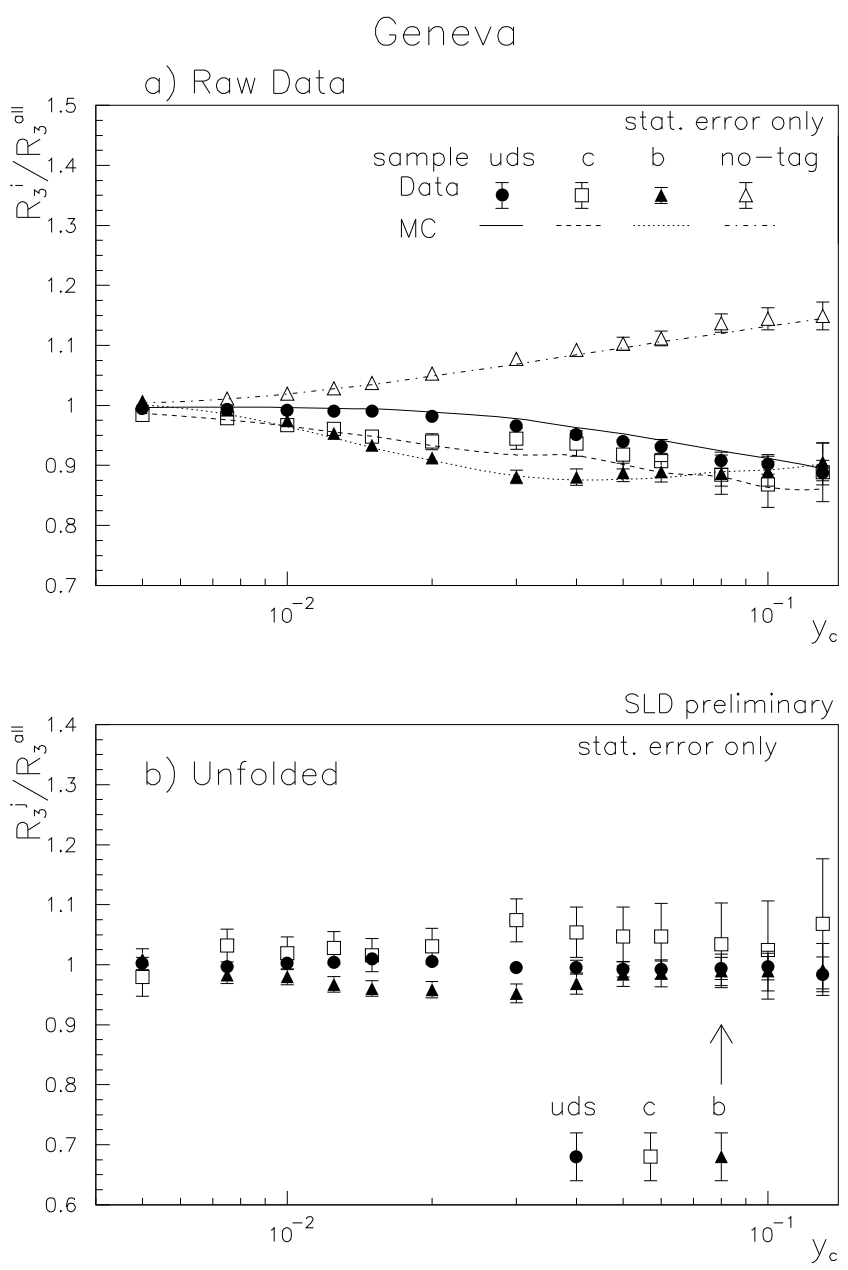

Figure 5.9: (a) The raw measured ratios $R_{3}^{j} / R_{3}^{\text {all }}$ vs. $y_{\text {cut }}$ for subsample $j$ (see text); data (points with error bars), and simulation (lines joining values at the same $y_{\text {cut }}$ values as the data). (b) The unfolded ratios $R_{3}^{i} / R_{3}^{\text {all }}, i=u d s, c, b$, vs. $y_{c}$ for the 3 primary event flavors. The arrow indicates the chosen $y_{c u t}$ value. : Geneva-algorithm 

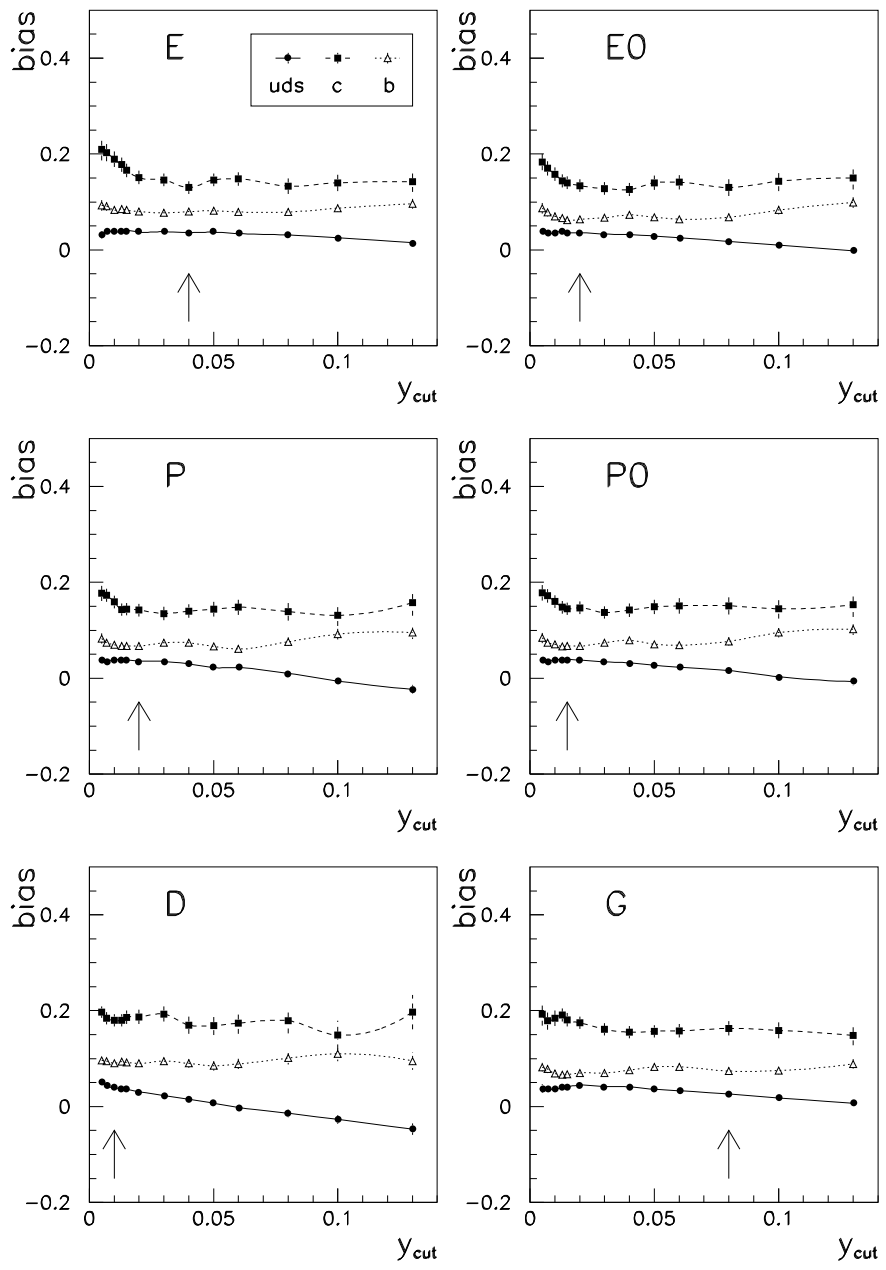

Figure 5.10: The flavor tag bias against 3-jet as a function of $y_{c u t}$. Arrows indicate the chosen $y_{c u t}$ values.

\subsection{Systematic Errors on $R_{3}^{i} / R_{3}^{\text {all }}$}

In the following sections, we will discuss the systematic errors that potentially affect our measurement of $R_{3}^{i} / R_{3}^{\text {all }}$. These may be divided into uncertainties in modeling the detector and uncertainties on experimental measurements serving as input parameters to the underlying physics modeling. The measurements rely on a Monte Carlo simulation to evaluate the efficiency matrices $\epsilon$ used in the procedure for unfolding from detector-level measured jet rates to those of the parton level. A large number of measured quantities from existing experiments relating to the production and decay of charm and bottom hadrons are used as input to our simulation based on the JETSET7.4 [56] event generator. The $B$ hadron decays are modeled according to the CLEO B decay model [90] tuned to reproduce the spectra measured at $\Upsilon(4 s)[91]$.

For each source of systematics considered, the error was evaluated by varying the appropriate parameter in the Monte Carlo simulation, turning on/off the corrections to the detector modeling, recalculating the unfolding matrices $\epsilon$, performing a new fit of Eq. 5.11 to the data, re-deriving values of $R_{3}^{i} / R_{3}^{\text {all }}$, and then taking the respective difference in results relative to our standard procedure as the systematic uncertainty.

\subsubsection{Detector Modeling}

We considered several properties of the detector modeling in the SLD Monte Carlo that potentially affect our measurement. The final results for $\alpha_{s}$ ratio are derived after applying all of the corrections discussed below.

\section{Tracking Efficiency}

Any variation of the number of tracks available for use could have some effect. The average multiplicities for the well-measured tracks are 19.90 in data and 20.34 in MC, those for the flavor-tagging tracks are 11.97 in data and 12.4 in MC. The former difference comes from the CDC tracking efficiency $96.67 \pm 0.35$ for data [109] and $97.47 \pm 0.07$ for MC [109], the latter predominantly comes from VXD hit linking efficiencies. We have applied a momentum dependent correction for the well-measured tracks, and a momentum and polar-angle dependent correction to the MC by tossing MC tracks randomly in order to provide good 
CHAPTER 5. MEASUREMENT OF THE $\alpha_{S}($ FLAVOR) RATIOS
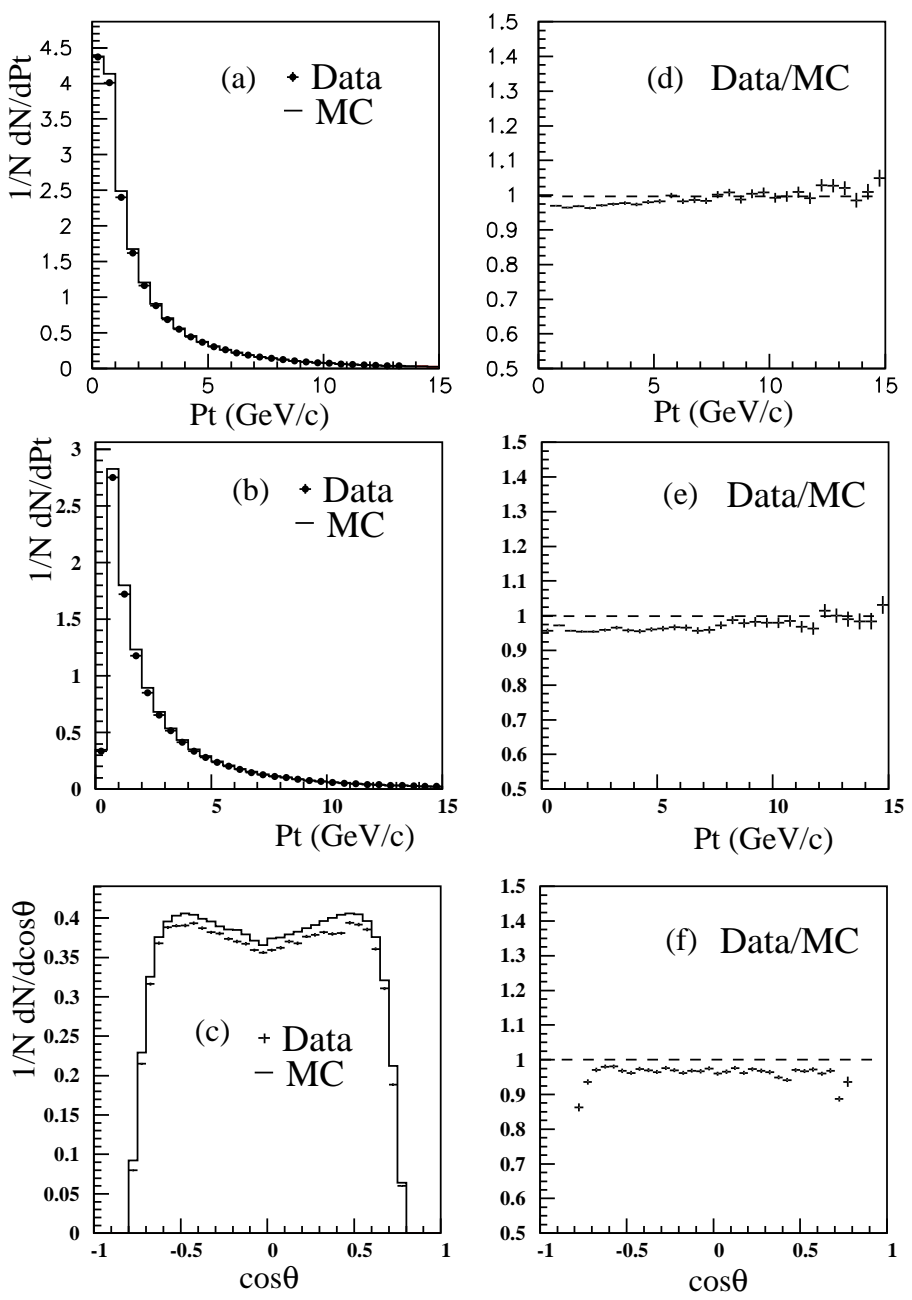

Figure 5.11: (a) Transverse momentum (w.r.t beam axis) distribution for the well-measured tracks, (b) Transverse momentum (w.r.t beam axis) distribution for the flavor tagging quality tracks, (c) Polar angle (w.r.t beam axis) distribution for the flavor tagging quality tracks, for data (points) and Monte Carlo (histogram), respectively. (d-f); Data/MC ratio for (a-c), respectively. agreement between the data and the MC. The distributions of transverse momentum with respect to the beam axis for the well-measured tracks and the flavor tagging quality tracks are shown in Fig. 5.11(a-b), and the distribution of polar angle with respect to the beam axis for the flavor tagging quality tracks in Fig. 5.11(c), as well as those ratios in Fig. 5.11(d-f).

The variation in the final results due to changing these corrections are relatively small.

\section{D impact parameter Resolution}

As described in Sect. 3.4.2, the modeling of the overall tracking resolution can be characterized by the impact parameter. The normalized 2-Dimensional impact parameter distribution of the flavor tagging quality tracks in the Monte Carlo describes the data quite well (Fig. 5.12a, also see Fig. 4.4), however, there is a small valley-shape structure in the core of the ratio between data and Monte Carlo (Fig. 5.12b). This implies that the modeling of the multiple scattering effect is slightly underestimated.

In order to well-match those of MC with data we hence smeared each flavor tagging tracks in the following manner:

$$
\frac{\delta^{x y}}{\sigma_{\delta^{x y}}}(\text { smeared })=\frac{\delta^{x y}}{\sigma_{\delta^{x y}}}(\mathrm{MC})+C\left(P_{t}, \cos \theta\right) \times G
$$

where $\delta^{x y} / \sigma_{\delta^{x} y}$ is the normalized 2D impact parameter, $G$ is the Gaussian random number with $\langle G\rangle=0$, and $C\left(P_{t}, \cos \theta\right)$ is the smearing factors taking into account transverse momentum w.r.t beam axis $P_{t}$ and polar angle $\theta$. The tracks with momentum $2.0<P_{t}<5.0$ $\mathrm{GeV}$ and large polar angle $0.6<\cos \theta<0.8$ in the MC were smeared by the factors $C\left(P_{t}, \cos \theta\right)$ listed in Table 5.4. These factors were estimated from the single Gaussian fit to the negative region of the $\delta^{x y} / \sigma_{\delta^{x y}}$ distribution, i.e resolution sensitive side. The smeared distribution is shown in Fig. 5.12c, as well as those ratio Fig. 5.12d. The difference between the result with smearing and without smearing was taken as a systematic error. 
Table 5.4: Smearing factors $C\left(P_{t}, \cos \theta\right)$ for 2-dimensional normalized impact parameter for MC.

\begin{tabular}{|c|cccc|}
\hline$P_{t} \backslash \cos \theta$ & $0.0-0.2$ & $0.2-0.4$ & $0.4-0.6$ & $0.6-0.8$ \\
\hline$<0.7 \mathrm{GeV}$ & 0. & 0. & 0. & 0.22572 \\
$0.7-2.0 \mathrm{GeV}$ & 0. & 0. & 0. & 0.33384 \\
$2.0-5.0 \mathrm{GeV}$ & 0.17631 & 0.16395 & 0.29100 & 0.52448 \\
$>5 \mathrm{GeV}$ & 0. & 0. & 0. & 0. \\
\hline
\end{tabular}
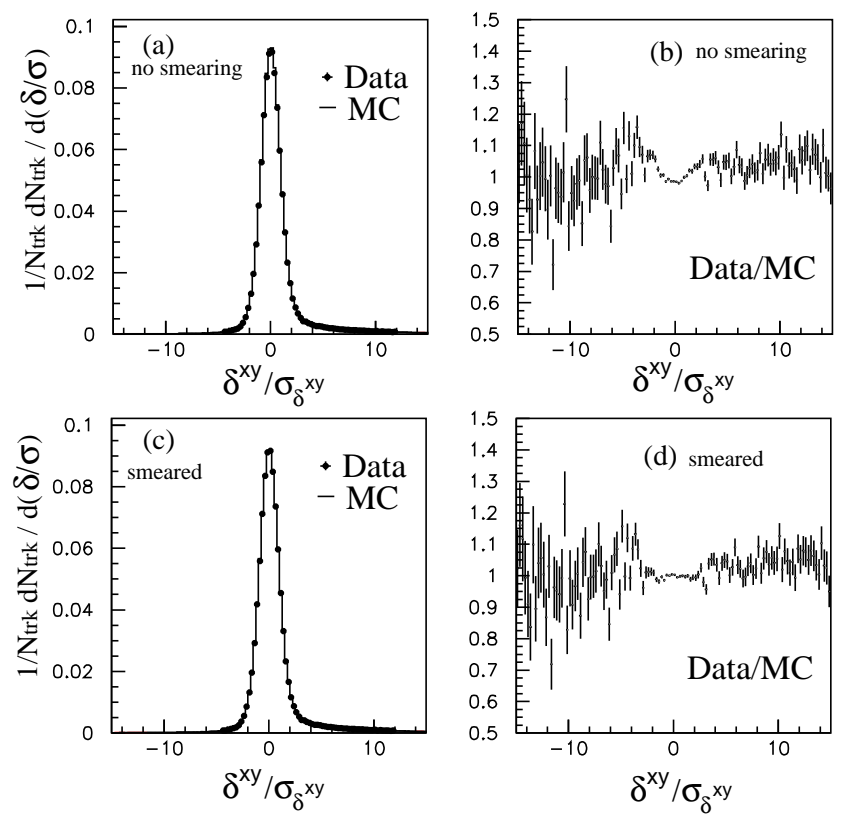

Figure 5.12: Distribution of the normalized 2D impact parameter for data (points) and Monte Carlo (histogram) comparing before smearing (a) and after smearing (c), and the corresponding ratios (b) and (d).

\section{$z$-coordinate track resolution}

Since this analysis uses the 3 dimensional topological reconstruction of the heavy hadron decay vertex to determine the initial quark flavor, we also investigated the $z$-coordinate tracking resolution. Figure 5.13(a) shows the data-Monte Carlo comparison for the normalized 3-D impact parameter $\delta^{3 D} / \sigma_{\delta}^{3 D}$. It should be noted that the dip at $\delta^{3 D} / \sigma_{\delta}^{3 D}=0$ in the distribution is due to reduced available phase space.

Just as described the $x y$ impact parameter is well described by MC, therefore this discrepancy predominantly seems to come from the resolution modeling for $z$-coordinate. In order to correct for the visible discrepancy (Fig. 5.13b), the $z$-coordinate of the helix parameter for each flavor tagging quality track was smeared:

$$
z_{\text {doca }}(\text { smeared })=z_{\text {doca }}(\mathrm{M} C)+\frac{g}{\cos \lambda},
$$

where $z_{\text {doca }}$ is the $z$-coordinate of the track at the distance of closest approach to the IP, $\lambda$ is the dip angle of the track and $g$ is a random number distributed according to the Gaussian probability with $\langle g\rangle=0$. This parameterization takes into account the dip angle dependence of the multiple scattering effect. Fig. 5.13 shows the distribution of $\delta^{3 D} / \sigma_{\delta}^{3 D}$ after smearing with $\sigma_{g}=20 \mu m[97,110,111]$. The ratio between data and MC is shown in Fig. 5.13d, the core distribution is well-described by the smeared MC. We took here the full correction as a systematic error. 

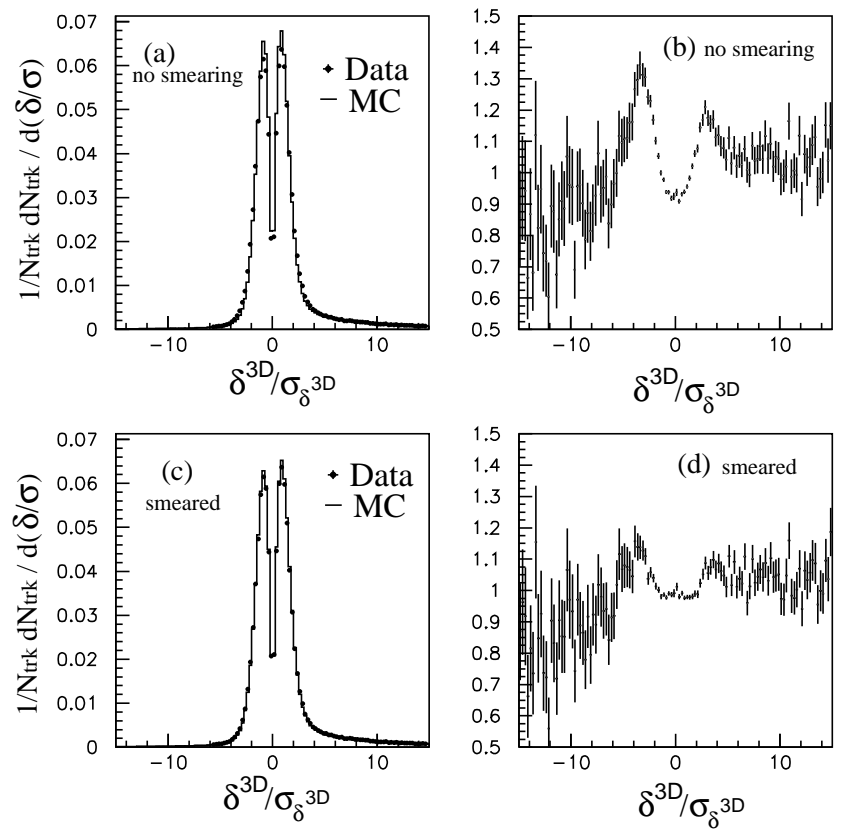

Figure 5.13: Distribution of the normalized 3D impact parameter for data (points) and Monte Carlo (histogram) comparing before smearing (a) and after smearing (c), and the corresponding ratios (b) and (d)

\subsubsection{MC statistics}

Since the efficiency matrices are evaluated using the MC, each matrix element has a statistical error due to the finite number of $\mathrm{MC}$ events available. We estimated the effects of this uncertainty by generating the multinomial distribution for each element of the matrices. Using the newly generated efficiency matrix, $R_{3}^{j} / R_{3}^{\text {all }}$ were re-evaluated. This procedure was repeated 1000 times for each algorithm. The root mean square derivations of the scattering around the central values of $R_{3}^{i} / R_{3}^{\text {all }}$ were taken as the errors due to finite MC statistics.

\subsubsection{Physics Modeling}

Since it is not feasible to generate a new set of Monte Carlo events and go through full detector simulation because of the limitation of the CPU power, systematic errors due to the uncertainties of physics modeling were studied by an event re-weighting method. One modified set of Monte Carlo events is obtained to reproduce a desired generator level distribution $G^{\text {mod. }}(\xi)$ of a parameter $\xi$ in question (e.g. $\xi=B$ lifetime) by weighting each event in the nominal Monte Carlo $G^{\text {nominal }}(\xi)$ with $W^{\text {mod. }}(\xi)$ :

$$
W^{\text {mod. }}(\xi)=\frac{G^{\text {mod. }}(\xi)}{G^{\text {nominal }}(\xi)} \text {. }
$$

We considered $B$ - and $D$-hadron properties that potentially affect our measurement. The central value and variation of each parameter were chosen to be consistent with the current world-averaged experimental measurement and its error.

\section{$B$ hadron Modeling}

\section{- $B$ fragmentation}

The mean scaled energy $\left\langle x_{E}\right\rangle_{B}=2 E_{B} / \sqrt{s}$ carried by weakly-decaying $B$ hadrons, where $E_{B}$ is the energy of a $B$ hadron and $\sqrt{s}$ is the center of mass energy, is varied as $\left\langle x_{E}\right\rangle_{B}=0.697 \pm 0.008$, which is consistent with measurements from SLD [66] and LEP $[64,112]$. The difference in function shapes between the Peterson model [63] and the Bowler model [62] with the same $\left\langle x_{E}\right\rangle_{B}$ was also investigated.

- $B$ hadron decay multiplicity 
The mean charged multiplicity 5.51 of $B$-hadron decays, which potentially affects SV reconstruction, is varied by \pm 0.35 tracks by re-weighting the multiplicity distribution. This variation is consistent with the SLD [113], LEP [114] and ARGUS [115] measurements.

- $B$ hadron lifetime

As the ease of tagging $b$ event is made possible by the long lifetimes of the $B$ hadron species, any change in the lifetimes may have an effect on the tagging efficiency. The values of $B$ meson (baryon) lifetimes in the MC samples are $1.56 \mathrm{ps}(1.10 \mathrm{ps})$. The $B$ meson lifetime was varied by $\pm 0.05 \mathrm{ps}$, and the $B$ baryon lifetime was variedby \pm 0.08 ps [20].

\section{- $B$ baryon production rate}

A change in the $B$ baryon production rate could affect the $b$-tagging efficiency due to its low lifetime. The rate of $B$ baryon production in $b$ events was changed from its central value of $7.6 \%$ by $\pm 3.2 \%[20]$.

\section{- $B \rightarrow D^{+}$fraction}

Changes in the fraction of $B \rightarrow D^{+}$decay could have some effect on the tagging efficiency for $b$ events, since the long decay path of the $D^{+}$downstream of the $B$ meson decay point enhances the efficiency for tagging that event. The branching fraction 0.192 is varied by $\pm 0.05[20]$.

- $R_{b}=B R\left(Z^{0} \rightarrow b \bar{b}\right) / B R\left(Z^{0} \rightarrow\right.$ hadrons $)$

The fraction of $b$ events in $Z^{0}$ decays gives the normalization for the unfolding procedure, so any change in that value has a direct effect on the values for the other quark flavors, due to the correlations in the unfolding. We varied $R_{b}$ by \pm 0.0017 about the Standard Model prediction of $R_{b}=0.2156$. This range of error is consistent with the current world average [20]. However, the high purity of the $b$-tag sample largely reduces the effects of these correlations.

\section{$D$-hadron Modeling}

- $R_{c}=B R\left(Z^{0} \rightarrow c \bar{c}\right) / B R\left(Z^{0} \rightarrow\right.$ hadrons $)$
As the $c$-tag is relatively low purity and contaminated by $b$ events, the fraction of $c$ events assumed for that sample has a potentially large effect on the outcome for $R_{3}^{c} / R_{3}^{\text {all }}$. This is due to the large background subtraction to derive $R_{3}^{c} / R_{3}^{\text {all }}$ at the parton level. We varied $R_{c}$ by \pm 0.010 about the standard model value of 0.172 [20].

- $D$ fragmentation

The mean scaled energy of charmed hadrons $\left\langle x_{E}\right\rangle_{D}=0.483$ was varied by \pm 0.008 , a value consistent with measurements from LEP [65]. The difference in function shapes between Peterson model [63] and the Bowler model [62] with the same mean value was also investigated.

- $D$ hadron lifetime

As well as $B$ hadron lifetime, the variation of $D$ hadron lifetime potentially affects the measurements. The values of $D$-meson $\left(\Lambda_{c}\right.$-baryon) lifetimes in the MC samples are varied according to the current world average [20].

- $c \bar{c} \rightarrow D+X$ fraction

Due to the long $D^{+}$lifetime, we would expect that the probability to find the secondary charm vertices depends on the rate of $D^{+}$production in $c \bar{c}$ events. We also investigated $D_{s}$ and $\Lambda_{c}$ fractions. The fractions $0.259,0.113$, and 0.074 of the $D^{+}+X, D_{s}+X$, and $\Lambda_{c}+X$ were varied by $\pm 0.028, \pm 0.037 \pm 0.029$, respectively [20].

- $D$ decay multiplicity

The systematic errors due to uncertainties in charmed meson decay topology were estimated by changing the Monte Carlo $D$ decay charged multiplicity according to the uncertainty in experimental measurements [116]. The mean decay multiplicities 2.54, $2.48,2,62,2.79$ of the $D^{0}, D^{+}, D_{s}$, and $\Lambda_{c}$ are varied by $\pm 0.06, \pm 0.06, \pm 0.31, \pm 0.45$, respectively.

- no- $\pi^{0}$ decay fraction

Charm decays with fewer neutral particles have higher charged mass and are therefore more likely to be tagged. Thus, an additional systematic uncertainty is estimated by varying the rates of charm decays with no $\pi^{0}$ 's by $\pm 10 \%$. 


\section{other physics modeling}

- The rate for $g \rightarrow Q \bar{Q}$

A rate for gluons to split into heavy quarks could change the frequency that $u d s$ events are tagged as heavy quark events. The $g \rightarrow b \bar{b}$ and $g \rightarrow c \bar{c}$ production rates are varied based on the OPAL $g \rightarrow c \bar{c}$ measurement; $f(g \rightarrow c \bar{c})=2.38 \pm 0.48 \times 10^{-2}[117]$ and the theoretical prediction for the ratio $f(g \rightarrow b \bar{b}) / f(g \rightarrow c \bar{c})=0.13$ [112]. This has a relatively small effect on the results.

- suppression for $s \bar{s}$ from vacuum

Uncertainties in the production of long-lived strange particles could also affect the measurements. The multiplicities of $K^{0}$ and $\Lambda$ were varied correspond to the strange quark suppression factor $0.280 \pm 0.028$, which is the ratio of the probability of popping up $s \bar{s}$ from the vacuum to that of $u \bar{u}$ or $d \bar{d}$. The central value and variation is consistent with LEP [118] and SLD [119] measurements.

For each algorithm at chosen $y_{\text {cut }}$ the errors on $R_{3}^{\text {uds }} / R_{3}^{\text {all }}, R_{3}^{c} / R_{3}^{\text {all }}$, and $R_{3}^{b} / R_{3}^{\text {all }}$ are listed in Table 5.6-5.10. The dominant physics contributions to $\delta R_{3}^{b} / R_{3}^{\text {all }}$ result from limited knowledge of the $B$-hadron fragmentation functions and the average $B$ decay multiplicity. The uncertainty in $B R\left(Z^{0} \rightarrow c \bar{c}\right)$ and in the charmed hadron fragmentation function produce dominant variations in $R_{3}^{c} / R_{3}^{\text {all }}$ and $R_{3}^{\text {uds }} / R_{3}^{\text {all }}$. Contributions from $B$ hadron lifetimes, the fraction of $D^{+}$in $B$ meson decays, $b$-baryon production rates, and the charm hadron decay multiplicity are relatively small.

For each jet altorithm and $y_{c u t}$ value all of the errors were added in quadrature to obtain a total experimental systematic error on each $R_{3}^{i} / R_{3}^{\text {all }}$ ratio.

\subsubsection{Hadronization Uncertainties}

The intrinsically non-perturbative process by which quarks and gluons fragment into the observed final-state hadrons cannot currently be calculated in perturbative-QCD. Phenomenological models of hadronization have been developed over the past few decades and have been implemented in Monte Carlo event-generator programs to facilitate comparison with experimental data. We have used the models implemented in the JETSET 7.4 and HERWIG 5.9 [57] programs to study hadronization effects; these models have been extensively studied and tuned to provide a good description of detailed properties of hadronic final states in $e^{+} e^{-}$annihilation; for a review of studies at the $Z^{0}$ resonance see [120]. Our standard simulation based on JETSET 7.4 was used to evaluate the efficiency and purity of the eventflavor tagging, as described in Sect. 5.3.2, as well as for the study of experimental systematic errors described in previous sections.

We investigated hadronization uncertainties by calculating from the Monte Carlo-generated event sample, the ratios:

$$
r_{i}=\left(\frac{R_{3}^{i}}{R_{3}^{\text {all }}}\right)_{\text {parton }} /\left(\frac{R_{3}^{i}}{R_{3}^{\text {all }}}\right)_{\text {hadron }}
$$

where $i=u d s, c$ or, $b$, parton refers to the calculation of the quantity in brackets at the parton-level, and hadron refers to the corresponding hadron-level calculation using stable final-state particles.

We recalculated these ratios by changing in turn the parameters $Q_{0}$ and $\sigma_{q}$ in the JSETSET program ${ }^{1}$ and generating one million event samples. We also recalculated these ratios by using the HERWIG 5.9 program with default parameter settings. For each variation we evaluated the fractional deviation $\Delta r_{i}$ w.r.t. the standard value:

$$
\Delta r_{i}=\frac{r_{i}^{\prime}-r_{i}}{r_{i}}
$$

and the corresponding deviations on $R_{3}^{i} / R_{3}^{\text {all }}$. For each algorithm the deviations are listed in Table 5.6-5.10. These deviations were added in quadrature to define the systematic error on $R_{3}^{i} / R_{3}^{\text {all }}$ due to hadronization uncertainties.

${ }^{1} Q_{0}(\mathrm{GeV})$ controls the minimum virtual mass allowed for partons in the parton shower; we considered a variation around the central value, 1.0 , of ${ }_{-0.5}^{+1.0} . \sigma_{q}(\mathrm{GeV} / c)$ is the width of the Gaussian distribution used to assign transverse momentum, w.r.t the color field, to quarks and antiquarks in the fragmentation process; we considered a variation around the central value, 0.39 of \pm 0.04 . 
Table 5.5: Compilation of the systematic errors for the E algorithm and $y_{\text {cut }}=0.04$. The first column shows the error source, the second column the central value used, and the third column the variation considered. The remaining columns show the corresponding errors on the values of $R_{3}^{\text {uds }} / R_{3}^{\text {all }}, R_{3}^{c} / R_{3}^{\text {all }}$, and $R_{3}^{b} / R_{3}^{\text {all }}$; '+' ('-') denotes the error corresponding to the relevant positive (negative) parameter variation.

\begin{tabular}{|c|c|c|c|c|c|c|c|c|}
\hline \multicolumn{9}{|c|}{ E algorithm } \\
\hline source & $\begin{array}{l}\text { Central } \\
\text { Value }\end{array}$ & Variation & \multicolumn{2}{|c|}{$\begin{array}{c}\delta R_{3}^{\text {uds }} / R_{3}^{\text {all }}(\%) \\
+\end{array}$} & \multicolumn{2}{|c|}{$\begin{array}{c}\delta R_{3}^{c} / R_{3}^{\text {all }}(\%) \\
+\end{array}$} & \multicolumn{2}{|c|}{$\begin{array}{c}\delta R_{3}^{b} / R_{3}^{\text {all }}(\%) \\
+\end{array}$} \\
\hline tracking efficiency & correction & off & 0.26 & & 0.60 & & -1.24 & \\
\hline 2D imp. par. res. & smear & off & 0.36 & & -2.72 & & 1.15 & \\
\hline $\mathrm{z}$ track resolution & smear & off & 0.11 & & -1.87 & & 1.18 & \\
\hline MC statistics & $0.8 \mathrm{M}$ & & 0.44 & -0.40 & 1.71 & -1.71 & 0.73 & -0.73 \\
\hline$B$ decay $\left\langle n_{c h}\right\rangle$ & 5.51 & \pm 0.35 trks & -0.34 & 0.44 & -0.80 & 0.20 & 1.61 & -1.40 \\
\hline$B$ fragm. $<x_{b}$ & 0.697 & \pm 0.008 & -0.47 & 0.50 & -0.39 & 0.49 & 1.65 & -1.80 \\
\hline$B$ fragm. shape & Peterson & Bowler & 0.35 & & 0.23 & & -1.19 & \\
\hline$B$ meson lifetime $\tau_{B}$ & $1.56 \mathrm{ps}$ & $\pm 0.05 \mathrm{ps}$ & 0.07 & -0.07 & -0.23 & 0.24 & -0.01 & 0.01 \\
\hline$B$ bary & 1. & $\pm 0.08 p s$ & -0.01 & 0.00 & -0.01 & 0.02 & 0.03 & -0.03 \\
\hline$B$ baryon proc & $7.6 \%$ & $\pm 3.2 \%$ & -0.10 & 0.10 & -0.12 & 0.15 & 0.38 & -0.39 \\
\hline$B \rightarrow D^{+}+$ & 0.192 & \pm 0.05 & 0.05 & 0.02 & -0.05 & -0.03 & -0.10 & -0.05 \\
\hline$Z^{0} \rightarrow b \bar{b}: f^{b}$ & 0.2156 & \pm 0.0017 & -0.10 & 0.10 & 0.17 & -0.17 & 0.11 & -0.11 \\
\hline$Z^{0} \rightarrow c \bar{c}: f^{c}$ & 0.172 & \pm 0.010 & \begin{tabular}{|l|}
-0.84 \\
\end{tabular} & 0.82 & 2.85 & -3.23 & -0.26 & 0.26 \\
\hline$C$ fragm. $\left\langle x_{c}\right\rangle$ & 0. & \pm 0.008 & -0.53 & 0.53 & 2.52 & -2.54 & -0.51 & 0.51 \\
\hline$C$ fragm. shape & Peterso & Bowler & 0.04 & & -0.17 & & 0.02 & \\
\hline$D^{0}$ decay $\left\langle n_{c h}\right\rangle$ & 2.54 & \pm 0.06 trks & -0.15 & 0.15 & 0.59 & -0.60 & -0.05 & 0.04 \\
\hline$D^{+}$decay $\left\langle n_{c h}\right\rangle$ & 2.48 & \pm 0.06 trks & -0.17 & 0.18 & 0.68 & -0.73 & -0.06 & 0.06 \\
\hline$D_{s}$ decay $\left\langle n_{c h}\right\rangle$ & 2.62 & \pm 0.31 trks & -0.26 & 0.27 & 0.96 & -0.98 & -0.01 & 0.00 \\
\hline$D^{0}$ lifetime & $0.418 p s$ & $\pm 0.004 \mathrm{ps}$ & 0.00 & 0.00 & 0.00 & 0.00 & 0.00 & 0.00 \\
\hline$D^{+}$lifetime & $1.054 \mathrm{ps}$ & $\pm 0.015 \mathrm{ps}$ & 0.00 & 0.00 & 0.01 & -0.01 & -0.01 & 0.02 \\
\hline$D_{s}$ lifetime & $0.466 \mathrm{ps}$ & $\pm 0.017 \mathrm{ps}$ & 0.01 & -0.01 & 0.01 & 0.00 & -0.04 & 0.03 \\
\hline$D^{0} \rightarrow K^{0}$ mult. & 0.402 & \pm 0.059 & -0.28 & 0.28 & 1.04 & -1.04 & -0.05 & 0.02 \\
\hline$D^{+} \rightarrow K^{0}$ mult. & 0.644 & \pm 0.078 & -0.34 & 0.38 & 1.21 & -1.38 & 0.00 & 0.03 \\
\hline$D_{s} \rightarrow K^{0}$ mult. & 0.382 & \pm 0.057 & -0.04 & 0.04 & 0.12 & -0.12 & 0.02 & -0.02 \\
\hline$D^{0} \rightarrow n o \pi^{0}$ & & 77 & -0.16 & 0.16 & 0.46 & -0.53 & 0.09 & -0.04 \\
\hline$D^{+} \rightarrow n o \pi^{0}$ & 0.496 & \pm 0.050 & -0.11 & 0.12 & 0.11 & -0.15 & 0.22 & -0.22 \\
\hline$D_{s} \rightarrow n o \pi^{0}$ & & \pm 0.035 & 0.03 & -0.03 & -0.07 & 0.07 & -0.02 & 0.02 \\
\hline$c \bar{c} \rightarrow D^{+}+X$ frac. & 0.2 & \pm 0.028 & -0.08 & 0.10 & 0.38 & -0.44 & -0.06 & 0.07 \\
\hline$c \bar{c} \rightarrow D_{s}+X$ frac. & & \pm 0.037 & 0.09 & -0.09 & -0.50 & 0.48 & 0.13 & -0.13 \\
\hline$c \bar{c} \rightarrow \Lambda_{c}+X$ & 0.0 & \pm 0.029 & 0.12 & -0.11 & -0.50 & 0.48 & 0.07 & -0.07 \\
\hline$\Lambda_{c} \operatorname{dec} a$ & & \pm 0.4 & -0.1 & 0. & 0.25 & -0.23 & 0.32 & -0.33 \\
\hline$\Lambda_{c}$ lifetim & 0.21 & $\pm 0.011 \mathrm{ps}$ & 0.03 & -0.01 & -0.16 & 0.04 & 0.05 & 0.01 \\
\hline$g \rightarrow b b$ & & & 0 . & -0.10 & 0.08 & -0.09 & -0.34 & 0.35 \\
\hline & & $\pm 0.48 \%$ & 0. & -0.08 & -0.20 & 0.20 & -0.07 & 0.08 \\
\hline$K^{0}$ prodn. & 0.658 trks & \pm 0.050 trks & 0.33 & -0.32 & -0.61 & 0.56 & -0.45 & 0.45 \\
\hline$\Lambda$ prodn. & 0.124 & $\pm 0.008 \mathrm{tr}$ & 00 & -0.0 & -0.05 & 0.10 & -0.02 & 0.04 \\
\hline Total Exp. Syst. & & & 1.59 & -1.42 & 4.86 & -6.15 & 3.08 & -3.11 \\
\hline$\overline{\mathrm{Q} 0}$ & $\overline{1 \mathrm{Ge}}$ & $\overline{-0}$ & \begin{tabular}{l|l}
-0.27 \\
\end{tabular} & 0.12 & $\begin{array}{c}-0.05 \\
\end{array}$ & 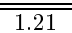 & 0.80 & \begin{tabular}{c|}
-1.34 \\
\end{tabular} \\
\hline & $0.39 \mathrm{GeV}$ & $\pm 0.04 \mathrm{GeV}$ & -0.04 & 0.05 & 0.27 & 0.01 & -0.09 & -0.13 \\
\hline hadronization model & JETSET7.4 & HERWIG5.9 & 0.91 & & 1.00 & & -3.44 & \\
\hline Total Hadronization & & & 0.91 & -0.27 & 1.59 & -0.05 & 0.80 & -3.69 \\
\hline
\end{tabular}

Table 5.6: Compilation of the systematic errors for the E0 algorithm and $y_{\text {cut }}=0.02$. The first column shows the error source, the second column the central value used, and the third column the variation considered. The remaining columns show the corresponding errors on the values of $R_{3}^{\text {uds }} / R_{3}^{\text {all }}, R_{3}^{c} / R_{3}^{\text {all }}$, and $R_{3}^{b} / R_{3}^{\text {all }}$; '+' ('-') denotes the error corresponding to the relevant positive (negative) parameter variation.

\begin{tabular}{|c|c|c|c|c|c|c|c|c|}
\hline \multicolumn{9}{|c|}{ E0 algorithm } \\
\hline source & $\begin{array}{l}\text { Central } \\
\text { Value }\end{array}$ & Variation & $\begin{array}{c}\delta R_{3}^{u d s} / \\
+\end{array}$ & , & $\begin{array}{c}\delta R_{3}^{c} / R \\
+\end{array}$ & $\pi(\%)$ & $\begin{array}{c}\delta R_{3}^{b} / H \\
+\end{array}$ & all $(\%)$ \\
\hline tracking efficiency & correction & off & 0.19 & & 0.40 & & -0.89 & \\
\hline 2D imp. par. res. & smear & off & $<0.01$ & & -0.91 & & 0.73 & \\
\hline $\mathrm{z}$ track resolution & smear & off & -0.26 & & -0.10 & & 0.83 & \\
\hline MC statistics & $0.8 \mathrm{M}$ & & 0.40 & -0.40 & 1.55 & -1.55 & 0.67 & -0.67 \\
\hline$B$ decay $\left\langle n_{c h}\right\rangle$ & 5.51 & \pm 0.35 trks & -0.23 & 0.32 & -0.54 & 0.08 & 1.08 & -0.96 \\
\hline$B$ fragm. $\left\langle x_{b}\right\rangle$ & 0.697 & \pm 0.008 & -0.35 & 0.39 & -0.38 & -0.46 & 1.31 & -1.47 \\
\hline$B$ fragm. shape & Peterson & Bowler & 0.41 & & 0.65 & & -1.69 & \\
\hline$B$ meson lifetime $\tau_{B}$ & $1.56 \mathrm{ps}$ & $\pm 0.05 \mathrm{ps}$ & 0.06 & -0.06 & -0.14 & 0.16 & -0.05 & 0.04 \\
\hline$B$ bary & & $\pm 0.08 \mathrm{ps}$ & $<0.01$ & $<0.01$ & -0.03 & 0.03 & 0.01 & -0.01 \\
\hline$B$ baryon prod & $7.6 \%$ & $\pm 3.2 \%$ & -0.07 & 0.08 & 0.07 & -0.08 & 0.13 & -0.15 \\
\hline$B \rightarrow D$ & 0.192 & \pm 0.05 & 0.01 & 0.04 & 0.12 & -0.08 & -0.12 & -0.04 \\
\hline$Z^{0} \rightarrow b \bar{b}: f^{b}$ & 0.2156 & \pm 0.0017 & -0.08 & 0.08 & 0.13 & -0.13 & 0.06 & -0.06 \\
\hline$Z^{0} \rightarrow c \bar{c}: f^{c}$ & 0.1 & \pm 0.010 & -0.62 & 0.61 & 1.98 & -2.24 & -0.23 & 0.23 \\
\hline$C$ fragm. $\left\langle x_{c}\right\rangle$ & 0.483 & \pm 0 . & .35 & 0.35 & 1.69 & -1.69 & -0.35 & 0.35 \\
\hline$C$ fragm. shape & Peterson & Bowler & -0.08 & & 0.32 & & -0.03 & \\
\hline$D^{0}$ decay $\left\langle n_{c h}\right\rangle$ & 254 & \pm 0.06 trks & -0.09 & 0.09 & 0.34 & -0.37 & -0.03 & 0.04 \\
\hline$D^{+}$decay $\left\langle n_{c h}\right\rangle$ & & \pm 0.06 trks & -0.14 & 0.15 & 0.53 & -0.56 & -0.03 & 0.02 \\
\hline$D_{s}$ decay $\left\langle n_{c h}\right\rangle$ & 2. & $\pm 0.31 \mathrm{trks}$ & -0.06 & 0.06 & 0.32 & -0.33 & -0.10 & 0.09 \\
\hline$D^{0}$ lifetime & $0.418 p s$ & $\pm 0.004 \mathrm{ps}$ & 0.01 & $<0.01$ & $<0.00$ & 0.01 & -0.02 & $<0.01$ \\
\hline$D^{+}$lifetime & $1.054 \mathrm{ps}$ & $\pm 0.015 \mathrm{ps}$ & $<0.01$ & $<0.01$ & 0.01 & -0.01 & -0.01 & 0.01 \\
\hline$D_{s}$ lifetime & $0.466 p s$ & $\pm 0.017 \mathrm{ps}$ & 0.01 & -0.01 & 0.02 & -0.02 & -0.03 & 0.03 \\
\hline$D^{0} \rightarrow K^{0}$ mult. & 0.402 & \pm 0.059 & -0.20 & 0.20 & 0.64 & -0.65 & 0.05 & -0.05 \\
\hline$D^{+} \rightarrow K^{0}$ mult. & 0.644 & \pm 0 . & -0.22 & 0.25 & 0.76 & -0.90 & 0.03 & -0.01 \\
\hline$D_{s} \rightarrow K^{0}$ mult. & 0.382 & \pm 0.057 & -0.03 & 0.03 & 0.09 & -0.10 & $<0.01$ & $<0.01$ \\
\hline$D^{0} \rightarrow n o \pi^{0}$ & & \pm 0 & -0.18 & 0.20 & 0.47 & -0.53 & 0.14 & -0.14 \\
\hline$D^{+} \rightarrow n o \pi^{0}$ & 0.496 & \pm 0.050 & -0.08 & 0.09 & 0.07 & -0.08 & 0.19 & -0.19 \\
\hline$D_{s} \rightarrow n o \pi^{0}$ & & & 0.01 & -0.01 & -0.01 & 0.01 & -0.02 & 0.02 \\
\hline$c \bar{c} \rightarrow D^{+}+X$ frac. & 0.2 & \pm 0.028 & -0.05 & 0.05 & 0.23 & -0.27 & -0.04 & 0.04 \\
\hline$c \bar{c} \rightarrow D_{s}+X$ frac. & 0.1 & \pm 0 . & 0.04 & -0.03 & -0.21 & 0.16 & 0.06 & -0.05 \\
\hline$c \bar{c} \rightarrow \Lambda_{c}+X$ & 0.0 & \pm 0. & 0.08 & -0.07 & -0.40 & 0.35 & 0.08 & -0.08 \\
\hline$\Lambda_{c} \mathrm{deca}$ & 2. & \pm 0 & -0.06 & 0. & -0.03 & 0.03 & 0.18 & -0.18 \\
\hline$\Lambda_{c}$ lifetime & 0.21 & \pm 0.0 & 0.07 & -0.02 & -0.28 & 0.09 & 0.02 & -0.01 \\
\hline$g \rightarrow b b$ & & & 0.08 & -0 . & 0.10 & -0.10 & -0.30 & 0.30 \\
\hline & & \pm 0.4 & 00 & -0 . & -0.12 & 0.13 & -0.09 & 0.09 \\
\hline$K^{0}$ prodn. & 0.658 trks & \pm 0.050 trks & 0.21 & -0.20 & -0.28 & 0.23 & -0.38 & 0.37 \\
\hline$\Lambda$ prodn. & 0.124 & $\pm 0.008 \mathrm{t}$ & 0.03 & -0.03 & -0.03 & -0.05 & -0.06 & 0.05 \\
\hline Total Exp. Syst. & & & 1.14 & -1.11 & 3.46 & -3.80 & 2.27 & -2.78 \\
\hline 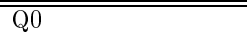 & $1 \mathrm{Ge}$ & $\overline{c+1-0}$ & 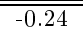 & "0.53 & $\overline{0.49}$ & 0.25 & 0.34 & $\begin{array}{ll}-1.79 \\
\end{array}$ \\
\hline & $0.39 \mathrm{G}$ & \pm 0.0 & -0.09 & $<0.01$ & 0.33 & -0.07 & 0.05 & 0.12 \\
\hline Total Hadronization & JETSET7.4 & HERWIG5.9 & 0.5 & & 1.74 & & -3.23 & \\
\hline Hadronization. Tota & & & 0.74 & -0.25 & 1.85 & -0.07 & 0.36 & -3.69 \\
\hline
\end{tabular}


Table 5.7: Compilation of the systematic errors for the $\mathrm{P}$ algorithm and $y_{\text {cut }}=0.02$. The first column shows the error source, the second column the central value used, and the third column the variation considered. The remaining columns show the corresponding errors on the values of $R_{3}^{\text {uds }} / R_{3}^{\text {all }}, R_{3}^{c} / R_{3}^{\text {all }}$, and $R_{3}^{b} / R_{3}^{\text {all }}$; '+' ('-') denotes the error corresponding to the relevant positive (negative) parameter variation.

\begin{tabular}{|c|c|c|c|c|c|c|c|c|}
\hline \multicolumn{9}{|c|}{$\mathrm{P}$ algorithm } \\
\hline source & Center & Variation & \multicolumn{2}{|c|}{$\begin{array}{c}\delta R_{3}^{\text {uds }} / R_{3}^{\text {all }}(\%) \\
+\end{array}$} & \multicolumn{2}{|c|}{$\delta R_{3}^{c} / R_{3}^{\text {all }}(\%)$} & \multicolumn{2}{|c|}{$\delta R_{3}^{b} / R_{3}^{\text {all }}(\%)$} \\
\hline tracking efficiency & correction & off & 029 & & 000 & & 000 & \\
\hline 2D imp. & smear & off & -0.1 & & -0.45 & & 0.60 & \\
\hline $\mathrm{z}$ trac & smea & off & -0.43 & & 0.67 & & 0.67 & \\
\hline MC statistics & $0.8 \mathrm{M}$ & & 0.35 & -0.35 & 1.38 & -1.38 & 0.62 & -0.62 \\
\hline$B$ decay $\left\langle n_{c h}\right\rangle$ & 5.51 & \pm 0.35 trks & -0.18 & 0.26 & -0.69 & 0.23 & 1.06 & -0.91 \\
\hline$B$ fragm. $<x_{b}$ & 0.697 & \pm 0.008 & -0.35 & 0.38 & -0.26 & 0.34 & 1.21 & -1.36 \\
\hline$B$ fragm. shape & Peterson & Bowler & 0.47 & & 0.34 & & -1.62 & \\
\hline$B$ meson lifetime $\tau_{B}$ & $1.56 \mathrm{ps}$ & $\pm 0.05 \mathrm{ps}$ & 0.06 & -0.06 & -0.15 & 0.16 & -0.04 & 0.04 \\
\hline$B$ bar & 1 & $\pm 0.08 p s$ & 0.00 & 0.00 & -0.03 & 0.03 & 0.01 & -0.01 \\
\hline$B$ bary & $6 \%$ & $\pm 3.2 \%$ & -0.09 & 0.11 & 0.15 & -0.19 & 0.15 & -0.16 \\
\hline$B \rightarrow D^{+}+$ & 0.192 & \pm 0.05 & 0.01 & 0.02 & 0.00 & 0.04 & -0.04 & -0.09 \\
\hline$Z^{0} \rightarrow b \bar{b}: f^{b}$ & 0.2156 & \pm 0.0017 & -0.07 & 0.07 & 0.13 & -0.13 & 0.06 & -0.06 \\
\hline$Z^{0} \rightarrow c \bar{c}: f^{c}$ & 0.172 & \pm 0.010 & \begin{tabular}{|c|}
-0.57 \\
\end{tabular} & 0.55 & 2.07 & -2.34 & -0.19 & 0.19 \\
\hline$C$ fragm. $\left\langle x_{c}\right\rangle$ & 0.4 & \pm 0.008 & -0.36 & 0.36 & 1.68 & -1.70 & -0.33 & 0.33 \\
\hline$C$ fragr & Peters & Bowler & -0.03 & & 0.12 & & 0.00 & \\
\hline$D^{0}$ decay $\left\langle n_{c h}\right\rangle$ & 2.54 & \pm 0.06 trks & -0.09 & 0.09 & 0.38 & -0.42 & -0.06 & 0.07 \\
\hline$D^{+}$decay $\left\langle n_{c h}\right\rangle$ & 2.48 & \pm 0.06 trks & -0.15 & 0.16 & 0.58 & -0.62 & -0.03 & 0.04 \\
\hline$D_{s}$ decay $\left\langle n_{c h}\right\rangle$ & 2.62 & \pm 0.31 trks & -0.16 & 0.17 & 0.59 & -0.61 & -0.02 & 0.02 \\
\hline$D^{0}$ lifetime & 0.41 & $\pm 0.004 \mathrm{ps}$ & 0.00 & 0.00 & 0.00 & 0.00 & -0.01 & 0.01 \\
\hline$D^{+}$lifetime & $54 p s$ & $\pm 0.015 \mathrm{ps}$ & 0.00 & 0.00 & 0.00 & 0.00 & -0.01 & 0.01 \\
\hline$D_{s}$ lifetime & $0.466 \mathrm{ps}$ & $\pm 0.017 \mathrm{ps}$ & 0.01 & -0.01 & 0.00 & 0.00 & -0.03 & 0.03 \\
\hline$D^{0} \rightarrow K^{0}$ mult. & 0.402 & \pm 0.059 & -0.24 & 0.25 & 0.71 & -0.73 & 0.13 & -0.14 \\
\hline$D^{+} \rightarrow K^{0}$ mult. & 0.644 & \pm 0.078 & -0.23 & 0.26 & 0.75 & -0.88 & 0.07 & -0.03 \\
\hline$D_{s} \rightarrow K^{0}$ mult. & 0.382 & \pm 0.057 & -0.04 & 0.04 & 0.16 & -0.17 & -0.01 & 0.01 \\
\hline$D^{0} \rightarrow n o \pi^{0}$ & & \pm & -0.09 & 0.10 & 0.25 & -0.31 & 0.06 & -0.03 \\
\hline$D^{+} \rightarrow n o \pi^{0}$ & 0. & \pm 0.050 & -0.10 & 0.10 & 0.11 & -0.11 & 0.18 & -0.19 \\
\hline$D_{s} \rightarrow n o \pi^{0}$ & & \pm & 0.02 & -0.02 & -0.05 & 0.05 & -0.02 & 0.02 \\
\hline$c \bar{c} \rightarrow D^{+}+X$ frac. & & \pm 0.028 & -0.03 & 0.04 & 0.15 & -0.19 & -0.03 & 0.04 \\
\hline$D+X$ frac & & \pm & 0.00 & 0.01 & -0.06 & 0.01 & 0.03 & -0.02 \\
\hline$c \bar{c} \rightarrow \Lambda_{c}+X$ & 0.0 & \pm 0.029 & 0.1 & -0.13 & -0.62 & 0.58 & 0.11 & -0.10 \\
\hline & & & -0. & & 0.19 & -0.18 & -0.02 & 0.01 \\
\hline$\Lambda_{c}$ lifetim & 0.21 & $\pm 0.011 \mathrm{ps}$ & 0.0 & -0.02 & -0.20 & 0.06 & 0.00 & 0.00 \\
\hline$g \rightarrow b b$ & & & $0.0 £$ & -0 . & 0.14 & -0.14 & -0.33 & 0.33 \\
\hline & & \pm 0 & 0. & -0 & -0.16 & 0.17 & -0.08 & 0.09 \\
\hline$K^{0}$ prodn. & $0.658 \mathrm{tt}_{1}$ & \pm 0.050 trks & 0.24 & -0.23 & -0.48 & 0.43 & -0.29 & 0.30 \\
\hline$\Lambda$ prodn. & 0.12 & \pm 0.008 & & -0.0 & 0.00 & 0.02 & -0.06 & 0.07 \\
\hline Total Exp. Syst. & & & 1.11 & -1.13 & 3.53 & -3.80 & 2.09 & -2.63 \\
\hline$\overline{\mathrm{Q} 0}$ & 16 & -0. & -0.14 & 0.51 & 0.51 & 0.18 & 0.02 & \begin{tabular}{c|}
-1.68 \\
\end{tabular} \\
\hline & $0.39 \mathrm{G}$ & $\pm 0.04 \mathrm{GeV}$ & 0.05 & -0.02 & -0.15 & 0.19 & $<0.01$ & -0.03 \\
\hline hadronization mod & JETSET7.4 & HERWIG5.9 & 0.02 & & 1.27 & & -1.35 & \\
\hline Total Hadronization & & & 0.51 & -0.14 & 1.39 & -0.15 & 0.02 & -2.16 \\
\hline
\end{tabular}

Table 5.8: Compilation of the systematic errors for the P0 algorithm and $y_{c u t}=0.015$. The first column shows the error source, the second column the central value used, and the third column the variation considered. The remaining columns show the corresponding errors on the values of $R_{3}^{\text {uds }} / R_{3}^{\text {all }}, R_{3}^{c} / R_{3}^{\text {all }}$, and $R_{3}^{b} / R_{3}^{\text {all }}$; '+' ('-') denotes the error corresponding to the relevant positive (negative) parameter variation.

\begin{tabular}{|c|c|c|c|c|c|c|c|c|}
\hline \multicolumn{9}{|c|}{ P0 algorithm } \\
\hline source & $\begin{array}{l}\text { Center } \\
\text { Value }\end{array}$ & Variation & $\begin{array}{c}\delta R_{3}^{u d s} / \\
+\end{array}$ & (\%) & $\begin{array}{c}\delta R_{3}^{c} / I \\
+\end{array}$ & $\begin{array}{c}{ }_{3}^{a l l}(\%) \\
-\end{array}$ & $\begin{array}{c}\delta R_{3}^{b} / 1 \\
+\end{array}$ & $\begin{array}{c}{ }^{n t l l}(\%) \\
-\end{array}$ \\
\hline tracking efficiency & correction & off & 0.32 & & -0.15 & & -0.79 & \\
\hline 2D imp. par. res. & smear & off & -0.04 & & -0.78 & & 0.74 & \\
\hline $\mathrm{z}$ track resolution & smear & off & -0.28 & & 0.07 & & 0.74 & \\
\hline MC statistics & $0.8 \mathrm{M}$ & & 0.32 & -0.32 & 1.16 & -1.16 & 0.55 & -0.55 \\
\hline$B$ decay $\left\langle n_{c h}\right\rangle$ & 5.51 & \pm 0.35 trks & -0.16 & 0.22 & -0.68 & 0.30 & 1.01 & -0.85 \\
\hline$B$ fragm. $\left\langle x_{b}\right\rangle$ & 0.697 & \pm 0.008 & -0.36 & 0.39 & -0.35 & 0.41 & 1.30 & -1.45 \\
\hline$B$ fragm. shape & Peterson & Bowler & 0.45 & & 0.36 & & -1.57 & \\
\hline$B$ meson lifetime $\tau_{B}$ & $1.56 \mathrm{ps}$ & $\pm 0.05 \mathrm{ps}$ & 0.06 & -0.05 & -0.12 & 0.14 & -0.07 & 0.04 \\
\hline$B$ baryon lifetime $\tau_{B}$ & $1.10 \mathrm{ps}$ & $\pm 0.08 \mathrm{ps}$ & 0.00 & 0.00 & -0.03 & 0.03 & 0.02 & -0.02 \\
\hline$B$ baryon prod. & $7.6 \%$ & $\pm 3.2 \%$ & -0.06 & 0.07 & 0.17 & -0.20 & 0.05 & -0.05 \\
\hline$B \rightarrow D^{+}+X$ frac. & 0.192 & \pm 0.05 & 0.01 & 0.03 & 0.06 & 0.00 & -0.09 & -0.10 \\
\hline$Z^{0} \rightarrow b \bar{b}: f^{b}$ & 0.2156 & \pm 0.0017 & -0.07 & 0.07 & 0.11 & -0.11 & 0.05 & -0.06 \\
\hline$Z^{0} \rightarrow c \bar{c}: f^{c}$ & 0.172 & \pm 0.010 & -0.53 & 0.52 & 1.64 & -1.86 & -0.17 & 0.17 \\
\hline$C$ fragm. $\left\langle x_{c}\right\rangle$ & 0.483 & \pm 0.008 & -0.30 & 0.30 & 1.47 & -1.46 & -0.31 & 0.30 \\
\hline$C$ fragm. shape & Peterson & Bowler & -0.10 & & 0.38 & & -0.04 & \\
\hline$D^{0}$ decay $<n_{c h}>$ & 2.54 & $\pm 0.06 \mathrm{trks}$ & -0.08 & 0.08 & 0.29 & -0.32 & -0.01 & 0.03 \\
\hline$D^{+}$decay $\left\langle n_{c h}>\right.$ & 2.48 & \pm 0.06 trks & -0.12 & 0.13 & 0.49 & -0.52 & -0.04 & 0.04 \\
\hline$D_{s}$ decay $\left\langle n_{c h}>\right.$ & 2.62 & \pm 0.31 trks & -0.12 & 0.12 & 0.39 & -0.40 & 0.03 & -0.03 \\
\hline$D^{0}$ lifetime & $0.418 p s$ & $\pm 0.004 \mathrm{ps}$ & 0.00 & 0.00 & 0.01 & 0.00 & -0.02 & 0.00 \\
\hline$D^{+}$lifetime & $1.054 \mathrm{ps}$ & $\pm 0.015 \mathrm{ps}$ & 0.00 & 0.00 & 0.01 & -0.01 & -0.01 & 0.01 \\
\hline$D_{s}$ lifetime & $0.466 \mathrm{ps}$ & $\pm 0.017 p s$ & 0.00 & -0.01 & 0.01 & -0.01 & -0.02 & 0.03 \\
\hline$D^{0} \rightarrow K^{0}$ mult. & 0.402 & \pm 0.059 & -0.14 & 0.14 & 0.45 & -0.47 & 0.04 & -0.04 \\
\hline$D^{+} \rightarrow K^{0}$ mult. & 0.644 & \pm 0.078 & -0.18 & 0.21 & 0.64 & -0.77 & 0.01 & 0.02 \\
\hline$D_{s} \rightarrow K^{0}$ mult. & 0.382 & \pm 0.057 & -0.02 & 0.02 & 0.08 & -0.08 & 0.00 & 0.00 \\
\hline$D^{0} \rightarrow n o \pi^{0}$ & 0.370 & \pm 0.037 & -0.10 & 0.13 & 0.29 & -0.36 & 0.07 & -0.07 \\
\hline$D^{+} \rightarrow n o \pi^{0}$ & 0.496 & \pm 0.050 & -0.11 & 0.11 & 0.19 & -0.18 & 0.16 & -0.17 \\
\hline$D_{s} \rightarrow n o \pi^{0}$ & 0.348 & \pm 0.035 & 0.01 & -0.01 & -0.01 & 0.01 & -0.02 & 0.03 \\
\hline$c \bar{c} \rightarrow D^{+}+X \mathrm{f}$ & 0.259 & \pm 0.028 & -0.05 & 0.06 & 0.24 & -0.27 & -0.05 & 0.05 \\
\hline$c \bar{c} \rightarrow D_{s}+X$ frac. & 0.113 & \pm 0.037 & 0.02 & -0.01 & -0.11 & 0.08 & 0.04 & -0.03 \\
\hline$c \bar{c} \rightarrow \Lambda$ & 0.074 & \pm 0.029 & 0.10 & -0.09 & -0.46 & 0.42 & 0.07 & -0.07 \\
\hline$\Lambda_{c}$ decay $\left\langle n_{c h}>\right.$ & 2.79 & \pm 0.45 trks & -0.05 & 0.05 & 0.06 & -0.06 & 0.11 & -0.10 \\
\hline$\Lambda_{c}$ lifetime & $0.216 \mathrm{ps}$ & $\pm 0.011 p s$ & 0.05 & -0.01 & -0.20 & 0.07 & 0.02 & -0.01 \\
\hline$g \rightarrow b b$ & 0.31 & $\pm 0.11 \%$ & 0.06 & -0.06 & 0.11 & -0.11 & -0.27 & 0.27 \\
\hline & 2.38 & $\pm 0.48 \%$ & 0.07 & -0.07 & -0.15 & 0.15 & -0.07 & 0.08 \\
\hline$K^{0}$ prodn. & 0.658 trks & \pm 0.050 trks & 0.23 & -0.21 & -0.32 & 0.29 & -0.39 & 0.37 \\
\hline$\Lambda$ prodn. & 0.124 trks & \pm 0.008 trks & 0.03 & -0.03 & -0.03 & 0.04 & -0.06 & 0.04 \\
\hline Total Exp. Syst. & & & 1.01 & -0.97 & 2.87 & -3.20 & 2.13 & -2.57 \\
\hline & $1 \mathrm{GeV}$ & $+1-0.5 \mathrm{GeV}$ & \begin{tabular}{c|c|}
-0.19 \\
\end{tabular} & 0.48 & 0.37 & 0.13 & 0.34 & \begin{tabular}{c|c|}
-1.56 \\
\end{tabular} \\
\hline & $0.39 \mathrm{GeV}$ & $\pm 0.04 \mathrm{GeV}$ & -0.17 & -0.02 & 0.41 & 0.11 & 0.22 & 0.05 \\
\hline hadron & JETSET7.4 & HERWIG5.9 & 0.31 & & 1.57 & & -2.59 & \\
\hline Total Hadronization. & & & 0.57 & -0.25 & 1.67 & $<0.01$ & 0.40 & -3.02 \\
\hline
\end{tabular}


Table 5.9: Compilation of the systematic errors for the Durham algorithm and $y_{c u t}=0.01$ The first column shows the error source, the second column the central value used, and the third column the variation considered. The remaining columns show the corresponding errors on the values of $R_{3}^{\text {uds }} / R_{3}^{\text {all }}, R_{3}^{c} / R_{3}^{\text {all }}$, and $R_{3}^{b} / R_{3}^{\text {all }}$; ' + ' ('-') denotes the error corresponding to the relevant positive (negative) parameter variation.

\begin{tabular}{|c|c|c|c|c|c|c|c|c|}
\hline \multicolumn{9}{|c|}{ Durham algorithm } \\
\hline source & $\begin{array}{l}\text { Center } \\
\text { Value }\end{array}$ & Variation & $\begin{array}{c}\delta R_{3}^{u d s} \\
+\end{array}$ & $\begin{array}{c}\text { Rall (\%) } \\
\text { a }\end{array}$ & $\begin{array}{c}\delta R_{3}^{c} / 1 \\
+\end{array}$ & all & $\begin{array}{c}\delta R_{3}^{b} / R \\
+\end{array}$ & $\begin{array}{l}\text { all } \\
\text { all } \\
-\end{array}$ \\
\hline tracking efficiency & correction & off & 0.57 & & -0.70 & & -1.06 & \\
\hline 2D imp. par. res. & smear & off & -0.15 & & -0.71 & & 1.01 & \\
\hline $\mathrm{z}$ track resolution & smear & off & -0.44 & & 0.48 & & 0.87 & \\
\hline MC statistics & $0.8 \mathrm{M}$ & & 0.45 & -0.45 & 1.81 & -1.81 & 0.74 & -0.74 \\
\hline$B$ decay $\left\langle n_{c h}\right\rangle$ & 5.51 & \pm 0.35 trks & -0.46 & 0.56 & -1.21 & 0.51 & 2.27 & -2.01 \\
\hline$B$ fragm. $\left\langle x_{b}\right\rangle$ & 0.697 & \pm 0.008 & -0.32 & 0.34 & -0.25 & 0.31 & 1.12 & -1.23 \\
\hline$B$ fragm. shape & Peterson & Bowler & 0.36 & & 0.07 & & -1.08 & \\
\hline$B$ meson lifetime $\tau_{B}$ & $1.56 \mathrm{ps}$ & $\pm 0.05 \mathrm{ps}$ & 0.07 & -0.09 & -0.23 & 0.22 & -0.02 & 0.07 \\
\hline$B$ bary & 1. & $\pm 0.08 p s$ & 0.02 & -0.02 & -0.08 & 0.07 & 0.01 & -0.01 \\
\hline$B$ baryon prod & $7.6 \%$ & $\pm 3.2 \%$ & -0.12 & 0.12 & 0.38 & -0.39 & 0.04 & -0.04 \\
\hline$B \rightarrow D^{+}+$ & 0.192 & \pm 0.05 & 0.05 & 0.02 & 0.15 & -0.21 & -0.25 & 0.12 \\
\hline$Z^{0} \rightarrow b \bar{b}: f^{b}$ & 0.2156 & \pm 0.0017 & -0.09 & 0.09 & 0.22 & -0.22 & 0.11 & -0.11 \\
\hline$Z^{0} \rightarrow c \bar{c}: f^{c}$ & 0.172 & \pm 0.010 & \begin{tabular}{|l|}
-0.91 \\
\end{tabular} & 0.89 & 3.13 & -3.56 & -0.29 & 0.29 \\
\hline$C$ fragm. $\left\langle x_{c}\right\rangle$ & 0.4 & \pm 0.0 & -0.49 & 0.50 & 2.23 & -2.30 & -0.39 & 0.40 \\
\hline$C$ fragm. shape & Peterso & Bowler & 0.04 & & -0.21 & & 0.06 & \\
\hline$D^{0}$ decay $\left\langle n_{c h}\right\rangle$ & 2.54 & \pm 0.06 trks & -0.16 & 0.16 & 0.66 & -0.64 & -0.08 & 0.05 \\
\hline$D^{+}$decay $\left\langle n_{c h}\right\rangle$ & 2.48 & \pm 0.06 trks & -0.22 & 0.23 & 0.83 & -0.89 & -0.04 & 0.06 \\
\hline$D_{s}$ decay $\left\langle n_{c h}\right\rangle$ & 2.62 & \pm 0.31 trks & -0.18 & 0.19 & 0.57 & -0.59 & 0.06 & -0.06 \\
\hline$D^{0}$ lifetime & $0.418 p s$ & $\pm 0.004 \mathrm{ps}$ & -0.01 & 0.00 & 0.00 & -0.02 & 0.02 & 0.02 \\
\hline$D^{+}$lifetime & $1.054 \mathrm{ps}$ & $\pm 0.015 \mathrm{ps}$ & 0.00 & 0.00 & 0.01 & -0.02 & 0.00 & 0.02 \\
\hline$D_{s}$ lifetime & $0.466 p s$ & $\pm 0.017 \mathrm{ps}$ & .00 & 0.00 & 0.05 & -0.05 & -0.05 & 0.05 \\
\hline$D^{0} \rightarrow K^{0}$ mult. & 0.402 & \pm 0.059 & -0.23 & 0.25 & 0.70 & -0.71 & 0.09 & -0.14 \\
\hline$D^{+} \rightarrow K^{0}$ mult. & 0.644 & \pm 0.078 & -0.35 & 0.37 & 1.05 & -1.19 & 0.14 & -0.09 \\
\hline$D_{s} \rightarrow K^{0}$ mult. & 0.382 & \pm 0.057 & -0.03 & 0.03 & 0.08 & -0.09 & 0.02 & -0.01 \\
\hline$D^{0} \rightarrow n o \pi^{0}$ & & & -0.15 & 0.14 & 0.48 & -0.51 & 0.04 & 0.01 \\
\hline$D^{+} \rightarrow n o \pi^{0}$ & 0.496 & \pm 0.050 & -0.22 & 0.23 & 0.49 & -0.50 & 0.24 & -0.24 \\
\hline$D_{s} \rightarrow n o \pi^{0}$ & & \pm & 0.03 & -0.03 & -0.05 & 0.06 & -0.04 & 0.03 \\
\hline$c \bar{c} \rightarrow D^{+}+X$ frac. & 0.2 & \pm 0.028 & -0.07 & 0.07 & 0.32 & -0.33 & -0.06 & 0.05 \\
\hline$c \bar{c} \rightarrow D_{s}+X$ frac. & 0.1 & \pm 0.037 & -0.01 & 0.00 & 0.00 & 0.04 & 0.03 & -0.04 \\
\hline$c \bar{c} \rightarrow \Lambda_{c}+X$ & 0.0 & \pm 0.029 & 0.20 & -0.19 & -0.87 & 0.83 & 0.13 & -0.14 \\
\hline$\Lambda_{c} \operatorname{dec} a$ & 2. & & -0 & 0.2 & 0.45 & -0.44 & 0.20 & -0.21 \\
\hline$\Lambda_{c}$ lifetime & 0.21 & \pm 0 . & 0.05 & -0.01 & -0.16 & 0.04 & -0.01 & 0.00 \\
\hline$g \rightarrow b b$ & & & 0.11 & -0.11 & 0.19 & -0.19 & -0.45 & 0.45 \\
\hline & & \pm 0 & 0.11 & -0.11 & -0.28 & 0.29 & -0.09 & 0.09 \\
\hline$K^{0}$ prodn. & 0.658 trks & \pm 0.050 trks & 0.23 & -0.24 & -0.61 & 0.54 & -0.18 & 0.24 \\
\hline$\Lambda$ prodn. & 0.124 & & & -0.0 & -0.04 & 0.11 & -0.04 & 0.10 \\
\hline Total Exp. Syst. & & & 1.61 & -1.53 & 4.89 & -5.44 & 3.08 & -3.01 \\
\hline$\overline{\mathrm{Q} 0}$ & $\overline{1 \mathrm{Ge}}$ & $+1-0.5$ & 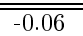 & 0.07 & 0.24 & 0.30 & \begin{tabular}{c|}
-0.04 \\
\end{tabular} & $2-0.50$ \\
\hline & $0.39 \mathrm{Ge}$ & $\pm 0.04 \mathrm{GeV}$ & -0.08 & 0.05 & 0.39 & -0.22 & -0.11 & 0.01 \\
\hline hadronization model & JETSET7.4 & HERWIG5.9 & 0.07 & & 0.14 & & -0.21 & \\
\hline Total Hadronization & & & 0.11 & -0.10 & 0.57 & -0.22 & 0.01 & -0.55 \\
\hline
\end{tabular}

Table 5.10: Compilation of the systematic errors for the Geneva algorithm and $y_{c u t}=0.08$ The first column shows the error source, the second column the central value used, and the third column the variation considered. The remaining columns show the corresponding errors on the values of $R_{3}^{u d s} / R_{3}^{\text {all }}, R_{3}^{c} / R_{3}^{\text {all }}$, and $R_{3}^{b} / R_{3}^{\text {all }} ;$ ' ' ' ' ('-') denotes the error corresponding to the relevant positive (negative) parameter variation.

\begin{tabular}{|c|c|c|c|c|c|c|c|c|}
\hline \multicolumn{9}{|c|}{ Geneva algorithm } \\
\hline source & $\begin{array}{l}\text { Center } \\
\text { Value }\end{array}$ & Variation & $\begin{array}{c}\delta R_{3}^{u d s} / \\
+\end{array}$ & (\%) & $\begin{array}{c}\delta R_{3}^{c} / H \\
+\end{array}$ & all & $\begin{array}{c}\delta R_{3}^{b} / R \\
+\end{array}$ & 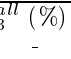 \\
\hline tracking efficiency & correction & off & 0.24 & & 0.76 & & -1.29 & \\
\hline 2D imp. par. res. & smear & off & 0.56 & & -3.20 & & 0.95 & \\
\hline $\mathrm{z}$ track resolution & smear & off & -0.25 & & -0.28 & & 0.93 & \\
\hline MC statistics & $0.8 \mathrm{M}$ & & 0.61 & -0.61 & 2.42 & -2.42 & 1.00 & -1.00 \\
\hline$B$ decay $\left\langle n_{c h}\right\rangle$ & 5.51 & \pm 0.35 trks & -0.47 & 0.53 & -0.24 & -0.64 & 1.51 & -1.00 \\
\hline$B$ fragm. $\left\langle x_{b}\right\rangle$ & 0.697 & \pm 0.008 & -0.31 & 0.33 & -0.04 & 0.15 & 0.92 & -1.04 \\
\hline$B$ fragm. shape & Peterson & Bowler & 0.49 & & -0.34 & & -1.12 & \\
\hline$B$ meson lifetime $\tau_{B}$ & $1.56 \mathrm{ps}$ & $\pm 0.05 p s$ & 0.10 & -0.11 & -0.33 & 0.32 & -0.01 & 0.07 \\
\hline$B$ baryon lifetime $\tau_{B}$ & $1.10 \mathrm{ps}$ & $\pm 0.08 \mathrm{ps}$ & 0.02 & -0.02 & -0.03 & 0.03 & -0.04 & 0.03 \\
\hline$B$ baryon prod. & $7.6 \%$ & $\pm 3.2 \%$ & -0.12 & 0.11 & 0.39 & -0.40 & 0.02 & -0.01 \\
\hline$B \rightarrow D^{+}+X$ frac. & 0.192 & \pm 0.05 & 0.01 & 0.05 & -0.18 & 0.03 & 0.12 & -0.16 \\
\hline$Z^{0} \rightarrow b \bar{b}: f^{b}$ & 0.2156 & \pm 0.0017 & -0.11 & 0.11 & 0.26 & -0.26 & 0.10 & -0.10 \\
\hline$Z^{0} \rightarrow c \bar{c}: f^{c}$ & 0.172 & \pm 0.010 & -0.99 & 0.97 & 3.40 & -3.86 & -0.30 & 0.30 \\
\hline$C$ fragm. $\left\langle x_{c}\right\rangle$ & 0.483 & \pm 0.008 & -0.50 & 0.50 & 2.23 & -2.21 & -0.36 & 0.36 \\
\hline$C$ fragm. shape & Peterson & Bowler & 0.03 & & -0.24 & & 0.10 & \\
\hline$D^{0}$ decay $\left\langle n_{c h}\right\rangle$ & 2.54 & \pm 0.06 trks & -0.09 & 0.09 & 0.41 & -0.34 & -0.07 & 0.01 \\
\hline$D^{+}$decay $\left\langle n_{c h}\right\rangle$ & 2.48 & \pm 0.06 trks & -0.18 & 0.19 & 0.90 & -0.97 & -0.20 & 0.23 \\
\hline$D_{s}$ decay $\left\langle n_{c h}\right\rangle$ & 2.62 & \pm 0.31 trks & -0.19 & 0.19 & 1.00 & -1.01 & -0.27 & 0.27 \\
\hline$D^{0}$ lifetime & $0.418 p s$ & $\pm 0.004 \mathrm{ps}$ & -0.02 & 0.01 & 0.03 & -0.05 & 0.03 & 0.02 \\
\hline$D^{+}$lifetime & $1.054 \mathrm{ps}$ & $\pm 0.015 \mathrm{ps}$ & -0.01 & 0.01 & 0.03 & -0.06 & 0.00 & 0.02 \\
\hline$D_{s}$ lifetime & $0.466 \mathrm{ps}$ & $\pm 0.017 \mathrm{ps}$ & 0.01 & -0.01 & 0.04 & -0.05 & -0.07 & 0.06 \\
\hline$D^{0} \rightarrow K^{0}$ mult. & 0.402 & \pm 0.059 & -0.32 & 0.34 & 1.21 & -1.18 & -0.07 & -0.03 \\
\hline$D^{+} \rightarrow K^{0}$ mult. & 0.644 & \pm 0.078 & -0.41 & 0.46 & 1.29 & -1.53 & 0.13 & -0.07 \\
\hline$D_{s} \rightarrow K^{0}$ mult. & 0.382 & \pm 0.057 & 0.00 & 0.00 & 0.02 & -0.03 & -0.02 & 0.01 \\
\hline$D^{0} \rightarrow n o \pi^{0}$ & 0.370 & \pm 0.037 & -0.22 & 0.19 & 0.73 & -0.71 & 0.03 & 0.04 \\
\hline$D^{+} \rightarrow n o \pi^{0}$ & 0.496 & \pm 0.050 & -0.19 & 0.19 & 0.43 & -0.44 & 0.18 & -0.18 \\
\hline$D_{s} \rightarrow n o \pi^{0}$ & 0.3 & \pm 0.035 & 0.05 & -0.05 & -0.13 & 0.14 & -0.04 & 0.03 \\
\hline$c \bar{c} \rightarrow D$ & 0.2 & \pm 0.0 & -0.15 & 0.14 & 0.61 & -0.57 & -0.07 & 0.05 \\
\hline$c \bar{c} \rightarrow D_{s}+X$ frac. & 0.1 & \pm 0.037 & -0.05 & 0.03 & 0.17 & -0.10 & -0.01 & 0.00 \\
\hline$c \bar{c} \rightarrow \Lambda_{c}+X$ & 0.074 & \pm 0.029 & 0.27 & -0.26 & -1.16 & 1.12 & 0.16 & -0.16 \\
\hline$\Lambda_{c}$ decay $<n_{c h}>$ & 2.7 & \pm 0.4 & -0.28 & 0.28 & 0.96 & -0.94 & 0.02 & -0.03 \\
\hline$\Lambda_{c}$ lifetime & $0.216 p s$ & $\pm 0.011 p s$ & 0.01 & 0.03 & -0.10 & -0.11 & 0.04 & 0.01 \\
\hline$g \rightarrow b b$ & 0.31 & & 0.1 . & -0.19 & 0.13 & -0.13 & -0.64 & 0.64 \\
\hline & 2.38 & $\pm 0.48 \%$ & 0.12 & -0.13 & -0.22 & 0.23 & -0.18 & 0.18 \\
\hline$K^{0}$ prodn. & 0.658 trks & \pm 0.050 trks & 0.31 & -0.32 & -1.02 & 0.98 & -0.08 & 0.14 \\
\hline$\Lambda$ prodn. & 0.124 trks & \pm 0.008 trks & 0.04 & -0.11 & -0.07 & 0.17 & -0.07 & 0.16 \\
\hline Total Exp. Syst. & & & 1.84 & -1.67 & 5.72 & -6.87 & 2.61 & -2.63 \\
\hline$\overline{\mathrm{QQ0}}$ & $\overline{1 \mathrm{Ge}}$ & $\overline{+1}$ & 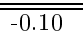 & 0.19 & 0.47 & 0.07 & 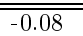 & 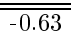 \\
\hline & $0.39 \mathrm{GeV}$ & $\pm 0.04 \mathrm{GeV}$ & 0.10 & -0.14 & -0.28 & -0.24 & -0.06 & 0.66 \\
\hline hadr & JETSET7.4 & HERWIG5.9 & -0.47 & & -0.04 & & 1.38 & \\
\hline Total Hadronization & & & 0.21 & -0.50 & 0.48 & -0.37 & 1.52 & -0.63 \\
\hline
\end{tabular}


Table 5.11: Central values and errors on $R_{3}^{i} / R_{3}^{\text {all }}$ for the 6 different jet-finding algorithms at the respective chosen $y_{c}$ values.

\begin{tabular}{|c|c|c|c|c|c|c|}
\hline & $R_{3}^{\text {uds }} / R_{3}^{\text {all }}$ & $R_{3}^{c} / R_{3}^{\text {all }}$ & $R_{3}^{b} / R_{3}^{\text {all }}$ & $R_{3}^{\text {uds }} / R_{3}^{\text {all }}$ & $R_{3}^{c} / R_{3}^{\text {all }}$ & $R_{3}^{b} / R_{3}^{\text {all }}$ \\
\hline & \multicolumn{3}{|c|}{$\mathrm{E}\left(y_{c}=0.04\right)$} & \multicolumn{3}{|c|}{ E0 $\left(y_{c}=0.02\right)$} \\
\hline Central val. & 0.982 & 1.025 & 1.031 & 0.978 & 1.042 & 1.030 \\
\hline Stat. & $\begin{array}{l}{ }_{-0.014}^{+0.014} \\
\end{array}$ & $\begin{array}{l}+0.050 \\
-0.050\end{array}$ & $\begin{array}{l}+0.022 \\
-0.022\end{array}$ & $\begin{array}{l}+0.010 \\
{ }_{-0.010}^{+0.0}\end{array}$ & $\begin{array}{l}+0.036 \\
-0.036\end{array}$ & $\begin{array}{l}0.016 \\
-0.016\end{array}$ \\
\hline Exp. Syst. & $\begin{array}{l}{ }_{-0.014}^{+0.016} \\
\end{array}$ & $\begin{array}{l}+0.049 \\
{ }_{-0.062}^{+0.04}\end{array}$ & ${ }_{-0.031}^{+0.031}$ & ${ }_{-0.011}^{+0.011}$ & $\begin{array}{l}+0.035 \\
{ }_{-0.038}^{+0.035} \\
\end{array}$ & $\begin{array}{l}+0.023 \\
{ }_{-0.028}\end{array}$ \\
\hline Hadronization & $\begin{array}{l}+0.009 \\
{ }_{-0.003}^{+0.09}\end{array}$ & $\begin{array}{l}0.016 \\
{ }_{-0.001}\end{array}$ & $\begin{array}{l}+0.008 \\
-0.037\end{array}$ & $\begin{array}{l}+0.007 \\
{ }_{-0.003}^{+}\end{array}$ & $\begin{array}{l}{ }_{-0.001}^{+0.019} \\
{ }_{-0.001}\end{array}$ & $\begin{array}{l}+0.004 \\
{ }_{-0.037}\end{array}$ \\
\hline \multirow[t]{2}{*}{ Total } & $\begin{array}{l}+0.023 \\
{ }_{-0.020}\end{array}$ & $\begin{array}{l}+0.072 \\
{ }_{-0.080}\end{array}$ & $\begin{array}{l}{ }_{-0.053}^{+0.039} \\
\end{array}$ & $\begin{array}{l}{ }_{-0.015}^{+0.0016} \\
\end{array}$ & $\begin{array}{l}{ }_{-0.052}^{+0.054} \\
\end{array}$ & $\begin{array}{l}{ }_{-0.049}^{+0.028} \\
\end{array}$ \\
\hline & \multicolumn{3}{|c|}{$\mathrm{P}\left(y_{c}=0.02\right)$} & \multicolumn{3}{|c|}{$\mathrm{P} 0\left(y_{c}=0.015\right)$} \\
\hline Central val. & 0.989 & 0.993 & 1.036 & 0.979 & 1.036 & 1.032 \\
\hline Stat. & $\begin{array}{l}+0.010 \\
{ }_{-0.010}\end{array}$ & $\begin{array}{l}+0.037 \\
{ }_{-0.037}\end{array}$ & $\begin{array}{l}+0.017 \\
{ }_{-0.017}^{+}\end{array}$ & $\begin{array}{l}+0.009 \\
{ }_{-0.009}^{+0}\end{array}$ & $\begin{array}{l}+0.032 \\
{ }_{-0.032}\end{array}$ & $\begin{array}{l}+0.014 \\
-0.014\end{array}$ \\
\hline Exp. Syst. & $\begin{array}{l}+0.011 \\
{ }_{-0.011}^{+}\end{array}$ & $\begin{array}{l}+0.035 \\
{ }_{-0.038}\end{array}$ & $\begin{array}{l}+0.021 \\
{ }_{-0.026}\end{array}$ & $\begin{array}{l}+0.010 \\
{ }_{-0.010}^{+0.0}\end{array}$ & $\begin{array}{l}+0.029 \\
{ }_{-0.032}\end{array}$ & $\begin{array}{l}+0.021 \\
{ }_{-0.026}\end{array}$ \\
\hline Hadronisation & $\begin{array}{l}+0.005 \\
{ }_{-0.001}^{+0.00}\end{array}$ & $\begin{array}{l}+0.014 \\
{ }_{-0.002}\end{array}$ & $\begin{array}{l}+0.0002 \\
{ }_{-0.022}\end{array}$ & $\begin{array}{l}{ }_{-0.003}^{+0.006} \\
\end{array}$ & ${ }_{-0.0001}^{+0.017}$ & $\begin{array}{l}{ }_{-0.030}+0.004 \\
\end{array}$ \\
\hline \multirow[t]{2}{*}{ Total } & $\begin{array}{l}+0.016 \\
{ }_{-0.015}\end{array}$ & $\begin{array}{l}+0.053 \\
{ }_{-0.053}\end{array}$ & $\begin{array}{l}+0.027 \\
{ }_{-0.038}\end{array}$ & $\begin{array}{l}+0.015 \\
{ }_{-0.014}\end{array}$ & $\begin{array}{l}+0.046 \\
{ }_{-0.045}\end{array}$ & $\begin{array}{l}+0.026 \\
{ }_{-0.042}\end{array}$ \\
\hline & \multicolumn{3}{|c|}{$\mathrm{D}\left(y_{c}=0.01\right)$} & \multicolumn{3}{|c|}{$\mathrm{G}\left(y_{c}=0.08\right)$} \\
\hline Central val. & 1.001 & 1.039 & 0.965 & 0.994 & 1.034 & 0.989 \\
\hline Stat. & $\begin{array}{l}+0.013 \\
{ }_{-0.013}^{+0.013} \\
\end{array}$ & $\begin{array}{l}+0.050 \\
-0.050\end{array}$ & $\begin{array}{l}+0.021 \\
{ }_{-0.021}^{+0.02}\end{array}$ & $\begin{array}{l}+0.018 \\
-0.018 \\
\end{array}$ & $\begin{array}{l}+0.069 \\
-0.069\end{array}$ & $\begin{array}{l}+0.028 \\
-0.028\end{array}$ \\
\hline Exp. Syst. & $\begin{array}{l}{ }_{-0.015}^{+0.016} \\
\end{array}$ & $\begin{array}{l}+0.049 \\
{ }_{-0.054}\end{array}$ & $\begin{array}{l}{ }_{-0.030}^{+0.031} \\
\end{array}$ & $\begin{array}{l}{ }_{-0.017}^{+0.018} \\
\end{array}$ & $\begin{array}{l}+0.057 \\
{ }_{-0.069}\end{array}$ & $\begin{array}{l}+0.026 \\
{ }_{-0.026}\end{array}$ \\
\hline Hadronisation & $\begin{array}{l}{ }_{-0.001}^{+0.001} \\
\end{array}$ & $\begin{array}{l}+0.006 \\
{ }_{-0.002}\end{array}$ & $\begin{array}{l}+0.0001 \\
-0.006\end{array}$ & $\begin{array}{l}+0.002 \\
{ }_{-0.005}\end{array}$ & $\begin{array}{l}+0.005 \\
{ }_{-0.004}\end{array}$ & $\begin{array}{l}+0.015 \\
{ }_{-0.006}\end{array}$ \\
\hline Total & $\begin{array}{l}{ }_{-0.020}^{+0.021} \\
\end{array}$ & $\begin{array}{l}+0.070 \\
-0.074 \\
\end{array}$ & $\begin{array}{l}+0.037 \\
-0.037\end{array}$ & $\begin{array}{l}+0.026 \\
{ }_{-0.025} \\
\end{array}$ & $\begin{array}{l}+0.090 \\
{ }_{-0.098} \\
\end{array}$ & $\begin{array}{l}+0.041 \\
-0.039 \\
\end{array}$ \\
\hline
\end{tabular}

\subsubsection{Choice of $y_{c u t}$ Values}

For each jet algorithm and $y_{c u t}$ value the statistical and experimental systematic errors and hadronization uncertainty on each $R_{3}^{i} / R_{3}^{\text {all }}$ were added in quadrature. No strong dependence of this combined error on $y_{c u t}$ was observed (Fig. 5.14-Fig. 5.19), but an 'optimal' $y_{c u t}$ value for each algorithm was then identified that corresponded with the smallest error. In the case of the $\mathrm{E}$ and $\mathrm{G}$ algorithms slightly larger $y_{c u t}$ values were chosen so as to ensure that the $\mathcal{O}\left(\alpha_{s}^{2}\right)$ calculations for massive quarks were reliable [121]. The chosen $y_{\text {cut }}$ value for each algorithm is listed in Table. 5.11, together with the corresponding values of the ratios $R_{3}^{i} / R_{3}^{\text {all }}$, as well as the statistical and experimental-systematic errors and hadronization uncertainties.
Figure 5.14: Row(a) statistical errors; (b) experimental systematic errors; (c) hadronization uncertainties; (d) total errors on $R_{3}^{i} / R_{3}^{\text {all }}$ as a function of $y_{\text {cut }}$ for $i=u d s$ (first column), $i=$ charm (second column), and $i=b$ flavors (third column), respectively, for E-algorithm. Arrows indicate the chosen $y_{c u t}$ values.
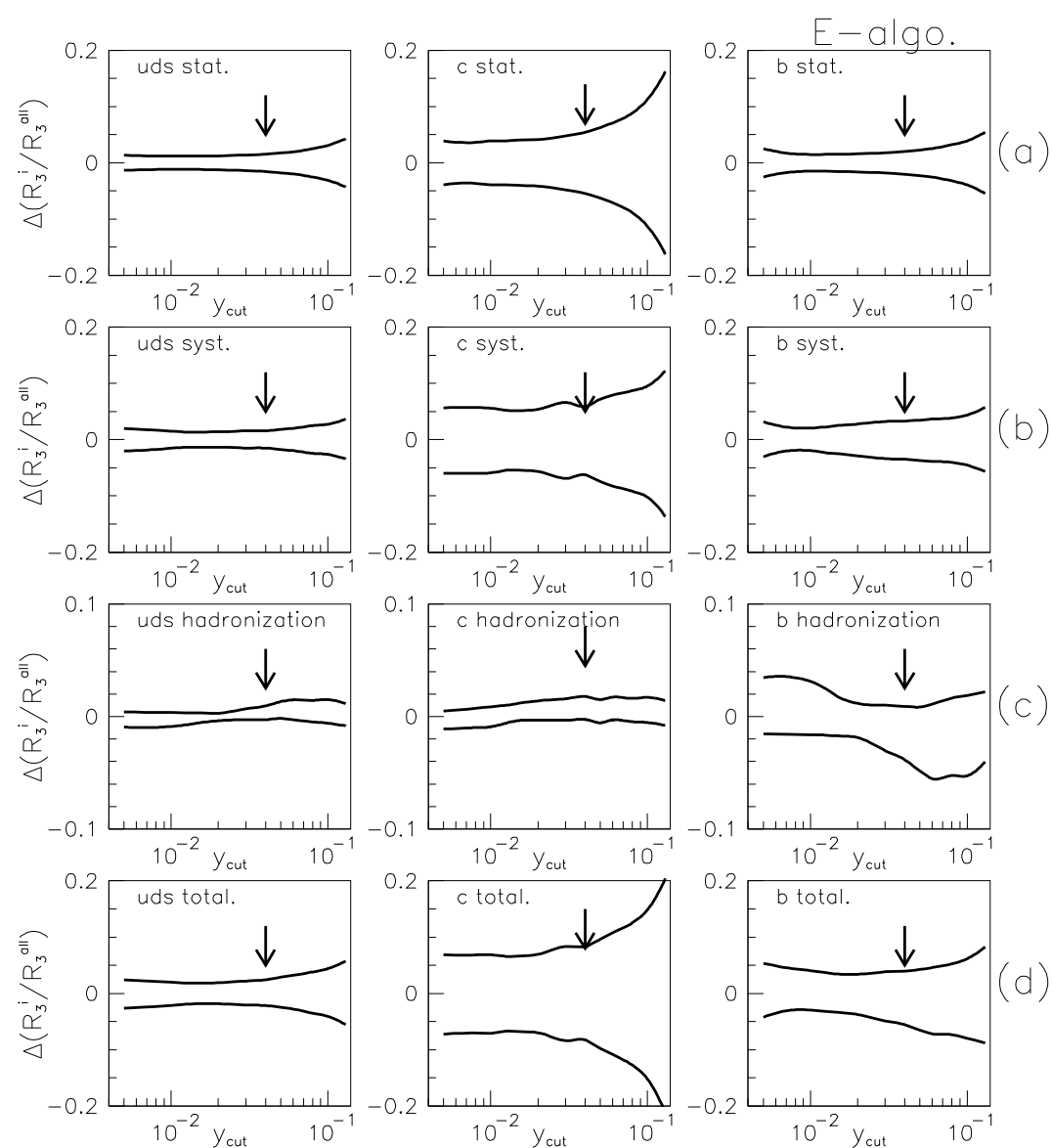
Figure 5.15: Row(a) statistical errors; (b) experimental systematic errors; (c) hadronization uncertainties; (d) total errors on $R_{3}^{i} / R_{3}^{\text {all }}$ as a function of $y_{\text {cut }}$ for $i=u d s$ (first column), $i=$ charm (second column), and $i=b$ flavors (third column), respectively, for E0-algorithm. Arrows indicate the chosen $y_{\text {cut }}$ values.
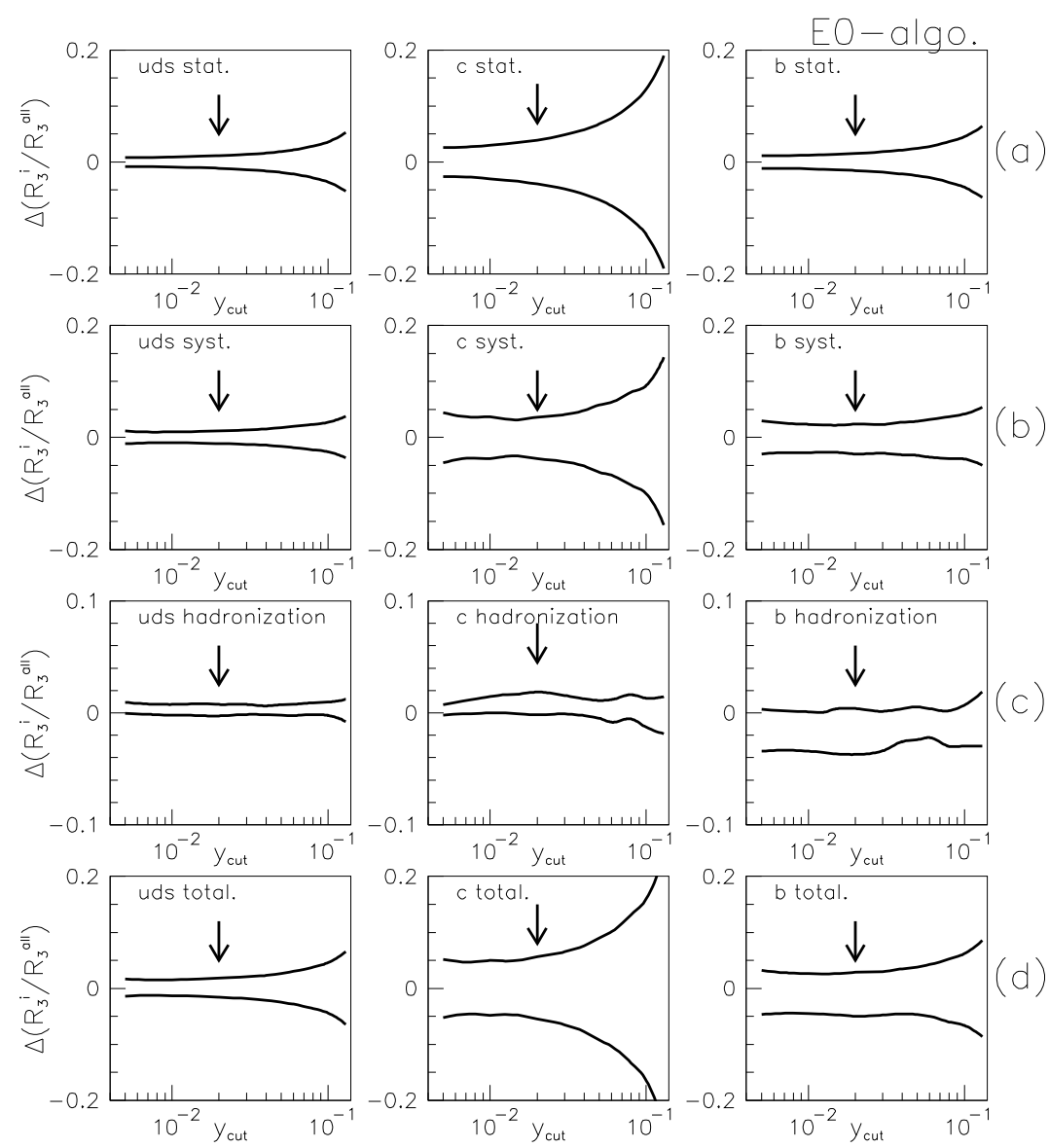

Figure 5.16: Row(a) statistical errors; (b) experimental systematic errors; (c) hadronization uncertainties; (d) total errors on $R_{3}^{i} / R_{3}^{a l l}$ as a function of $y_{c u t}$ for $i=u d s$ (first column), $i=$ charm (second column), and $i=b$ flavors (third column), respectively, for P-algorithm. Arrows indicate the chosen $y_{\text {cut }}$ values.
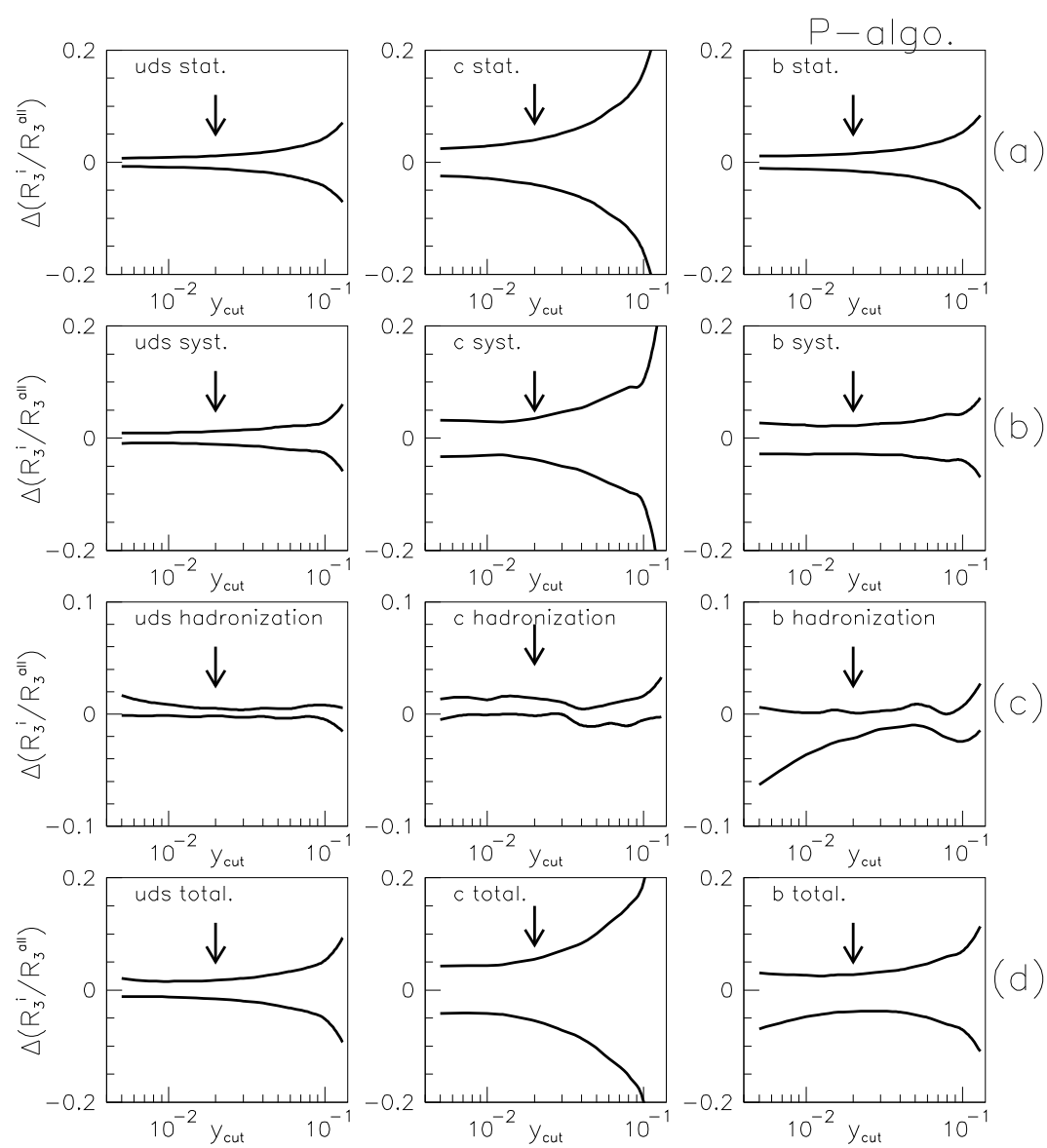
Figure 5.17: Row(a) statistical errors; (b) experimental systematic errors; (c) hadronization uncertainties; (d) total errors on $R_{3}^{i} / R_{3}^{\text {all }}$ as a function of $y_{\text {cut }}$ for $i=u d s$ (first column), $i=\operatorname{charm}$ (second column), and $i=b$ flavors (third column), respectively, for P0-algorithm. Arrows indicate the chosen $y_{\text {cut }}$ values.
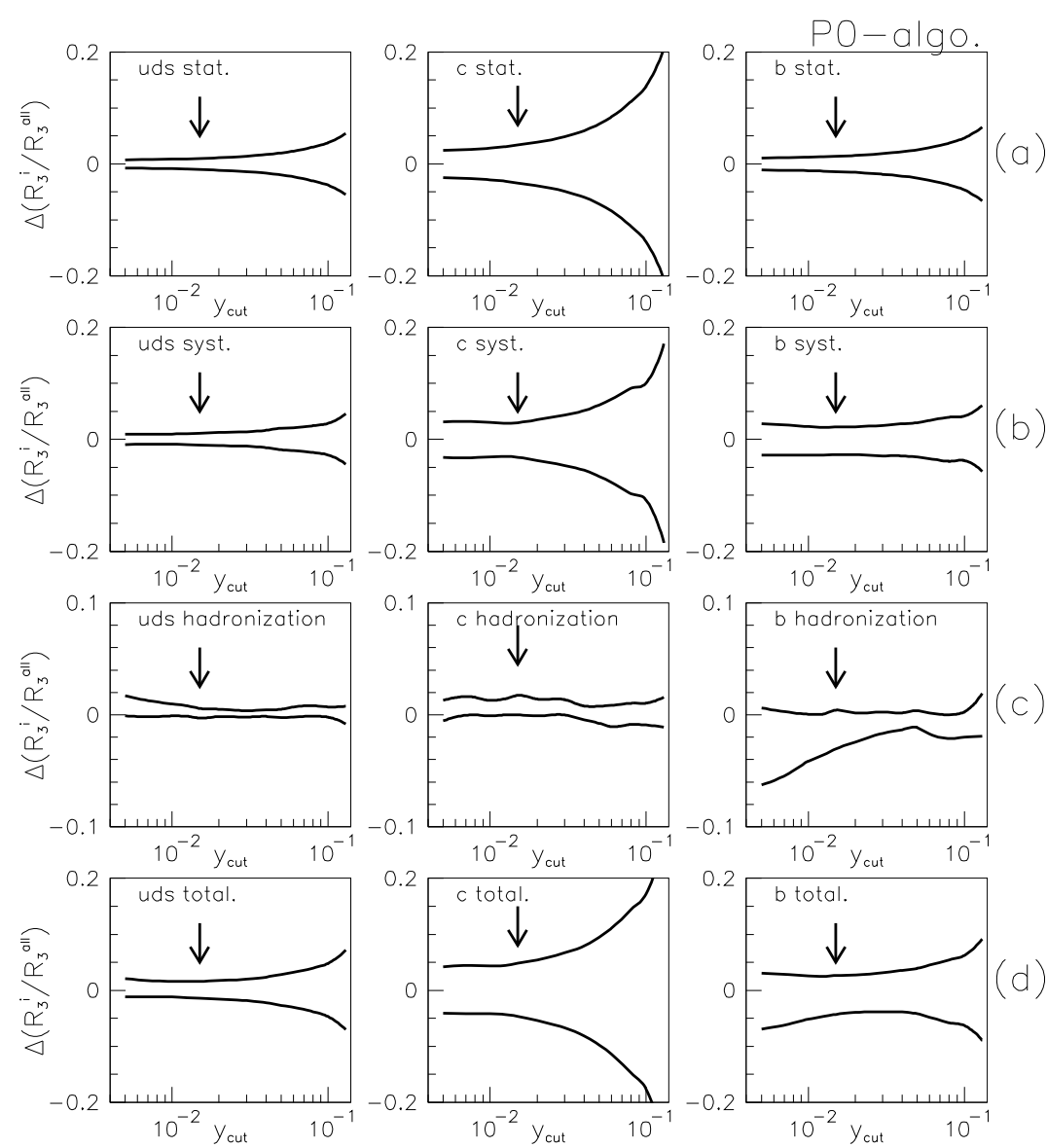

Figure 5.18: Row(a) statistical errors; (b) experimental systematic errors; (c) hadronization uncertainties; (d) total errors on $R_{3}^{i} / R_{3}^{\text {all }}$ as a function of $y_{\text {cut }}$ for $i=u d s$ (first column), $i=$ charm (second column), and $i=b$ flavors (third column), respectively, for Durham algorithm. Arrows indicate the chosen $y_{\text {cut }}$ values.
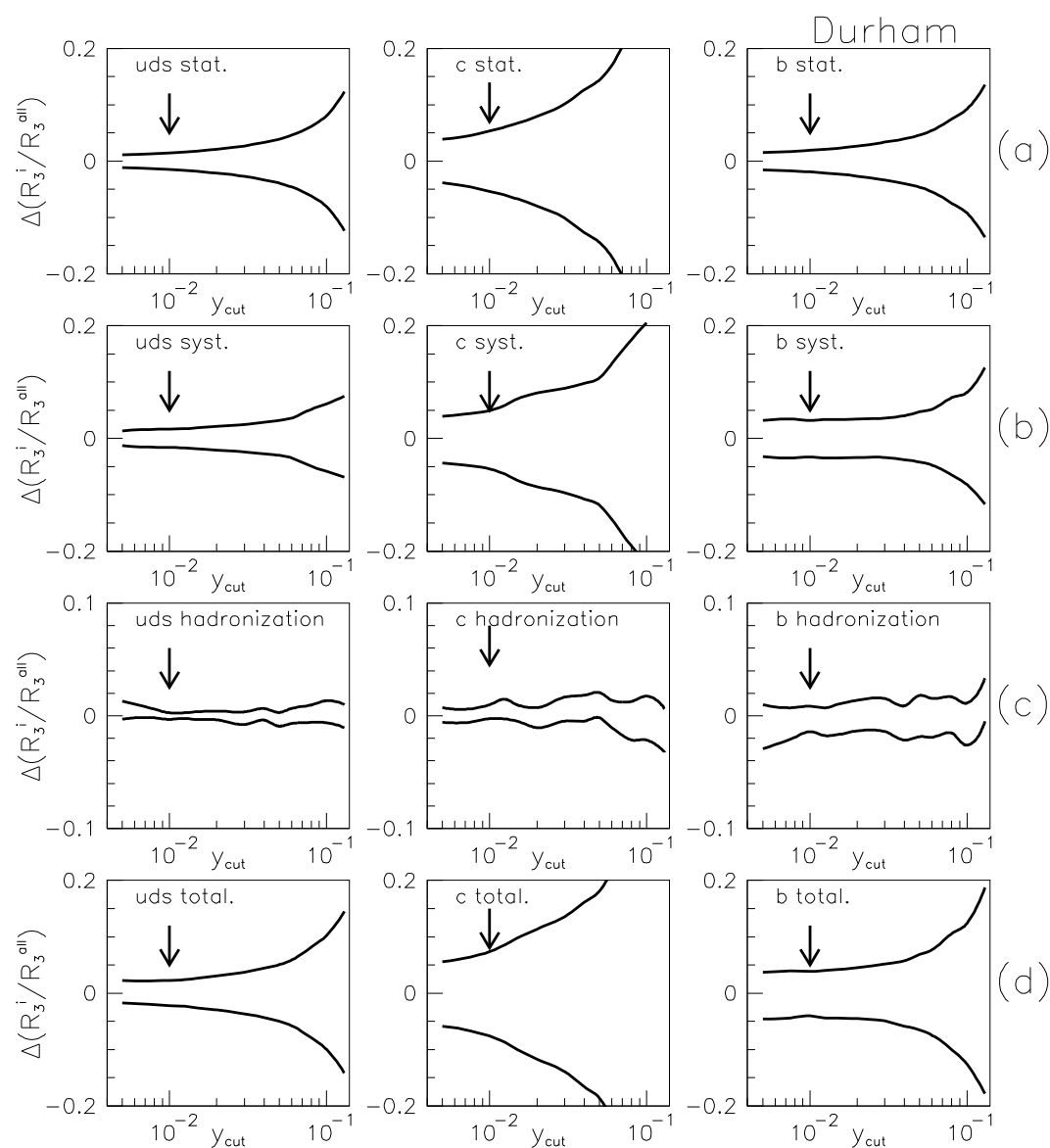
Figure 5.19: Row(a) statistical errors; (b) experimental systematic errors; (c) hadronization uncertainties; (d) total errors on $R_{3}^{i} / R_{3}^{\text {all }}$ as a function of $y_{\text {cut }}$ for $i=u d s$ (first column), $i=$ charm (second column), and $i=b$ flavors (third column), respectively, for Geneva algorithm. Arrows indicate the chosen $y_{c u t}$ values.
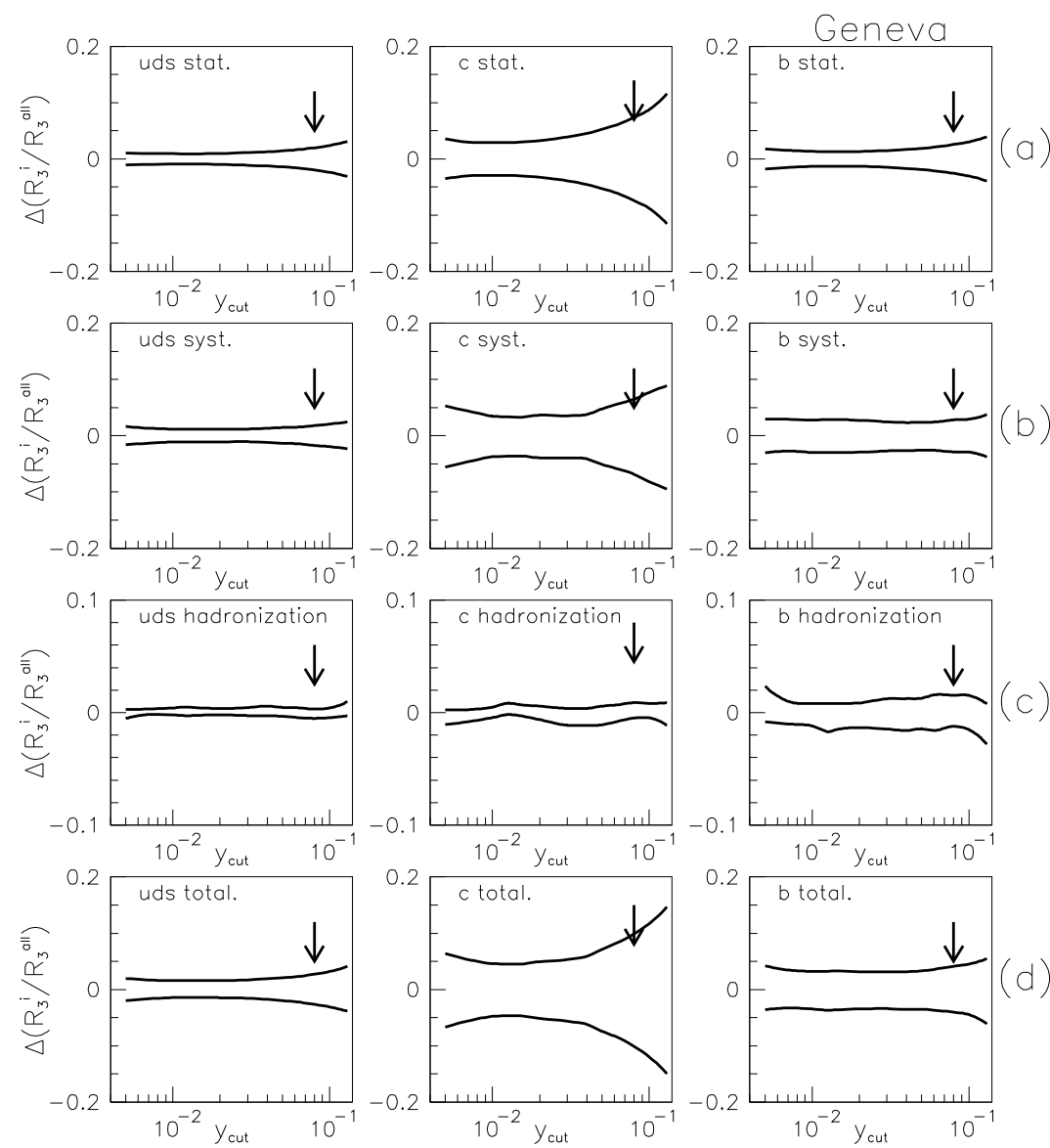

\subsection{Translation to $\alpha_{s}$ Ratios}

We then translate the values and errors on the measured $R_{3}$ ratios into the corresponding values and errors on the ratios of strong couplings [107]. This can be done using the relevant perturbative QCD calculations. With our definition, $R_{3}$ is expressed up to the Next-toLeading-Order (NLO) $\mathcal{O}\left(\alpha_{s}^{2}\right)$ in perturbative QCD :

$$
R_{3}^{i}=A^{i} \bar{\alpha}_{s}^{i}+\left(B^{i}+C^{i}\right)\left(\bar{\alpha}_{s}^{i}\right)^{2}
$$

where $\bar{\alpha}_{s}=\alpha_{s} / 2 \pi, A^{i}$ and $B^{i}$ are, respectively the Leading-Order (LO) and NLO contributions from 3 -jet final states, and $C^{i}$ is the LO contribution contribution from 4-jet final states. These coefficients depend implicitly upon the jet algorithm (see e.g. [31]) as well as on the scaled-invariant-mass-squared jet resolution parameter $y_{\text {cut }}$; for clarity these dependences have been omitted from the notation. For massless quarks we denote the coefficients $A^{0}, B^{0}$ and $C^{0}$, and calculations have been available for many years $[122,123]$. The values used are given in Table 5.12 .

\subsubsection{Heavy Quark Mass Effects}

At the $Z^{0}$ pole the quark mass often appears in terms proportional to the ratio $m_{b}^{2} / m_{Z}^{2}$, and the effects of non-zero quark mass can be neglected for many observables. For the 3 -jet rate, however, mass effects can enter via terms proportional to $m_{b}^{2} /\left(y_{\text {cut }} m_{Z}^{2}\right)$. For $b$-quarks these terms can contribute at the $\mathcal{O}(5 \%)$ level for typical values of $y_{c u t}$ used in jet clustering. Therefore, the 3-jet rate in heavy-quark events is expected to be modified relative to that in light-quark events by the diminished phase-space for gluon emission due to the quark mass, as well as by kinematic effects in the definition of the jet clustering schemes.

- Phase space suppression

The gluon radiation is suppressed by the diminished phase-space due to the quark masses $[124,125,126,127,128]$. In terms of parton shower evolution, a quark may radiate gluons until its virtual mass becomes $\sim m_{q}$ This is schematically shown in Fig. 5.21(a). The depletion of gluon radiation for $b$-quarks apparently diminishes the 3 -jet rate for $b$ events. 
- Jet-clustering invariant mass

As described in Sect. 5.2.1, two pseudo particles $i$ and $j$ are defined as two distinct jets if $m_{i j}^{2}>y_{\text {cut }} s$, where $m_{i j}$ is the invariant mass between two pseudo-particles and $\sqrt{s}$ is the center of mass energy.

For the same kinematic configuration, a $b$-quark jet is more likely to be identified as a individual jet whereas light quarks are more likely to be clustered with soft gluons. This is schematically shown in Fig. 5.21(b-c). Therefore jet clustering tends to enhance the jet rate of $b$ events relative to those of light flavors. The magnitude of the effects depends on the clustering algorithm and $y_{c u t}$.

This effect was first pointed out by Ioffe [124], and investigated by Ballestero, Maina, and Moretti $[125,126]$. Our previously published results were corrected using the relevant tree-level calculations [126].

Very recently two groups have completed next-to-leading-order $\left(\mathcal{O}\left(\alpha_{s}^{2}\right)\right)$ perturbative QCD calculations for massive-quark final states in the framework of the $\overline{M S}$ renormalization scheme. These calculations have been found to be in agreement with $[127,128]$

As well as the coupling constant $\alpha_{s}$, several valid renormalization schemes exist for quark masses, each one postulating a different renormalized mass definition. The perturbative pole mass $M_{q}$, and the running mass $m_{q}(\mu)$ renormalized at energy scale $\mu$ in the $\overline{M S}$ scheme are among the most attractive mass definitions due to their intrinsic physical properties. The former definition is the renormalized pole of the fermion propagator prescribed by the on-shell scheme. For free fermions, it corresponds to the usual kinematic mass reconstructed in experiments. The latter definition is purely dynamical, it is associated to the renormalized fermion mass of the $\overline{M S}$ scheme and depends on the energy scale $\mu$ of the process under study and is appropriate for this study. The $b$ quark mass has been extracted from the known spectra of the hadronic bound states of the $\Upsilon$ resonances using QCD sum rules or lattice calculations [129]: $M_{b} \approx 4.7 \mathrm{GeV} / c^{2}$ and $m_{b}\left(m_{b}\right)=4.2 \mathrm{GeV} / c^{2}$. As described in Sect. 2.4.1 the DELPHI collaboration recently measured the $b$ mass defined in the $\overline{M S}$ scheme from the 3-jet production rate at the $Z^{0}$ pole: $m_{b}\left(M_{Z}\right)=2.67 \pm 0.5($ total $)$ [71].

We used the results [121] of the calculations described in [128]. Values for $A^{b}, B^{b}, C^{b}$ for each jet algorithm are calculated using $m_{b}\left(M_{Z}\right)=3.0 \pm 0.5 \mathrm{GeV} / c^{2}$ for the six jet algorithms at $y_{c u t}$ values used in our study, and listed in Table. 5.13. As an example of heavy quark mass effects, the calculated ratio for $R_{3}^{b} / R_{3}^{0}$ for the Durham algorithm using $m_{b}\left(M_{Z}\right)=3.0 \mathrm{GeV}$ is shown in Fig. 5.20 as a function of $y_{\text {cut }}$.

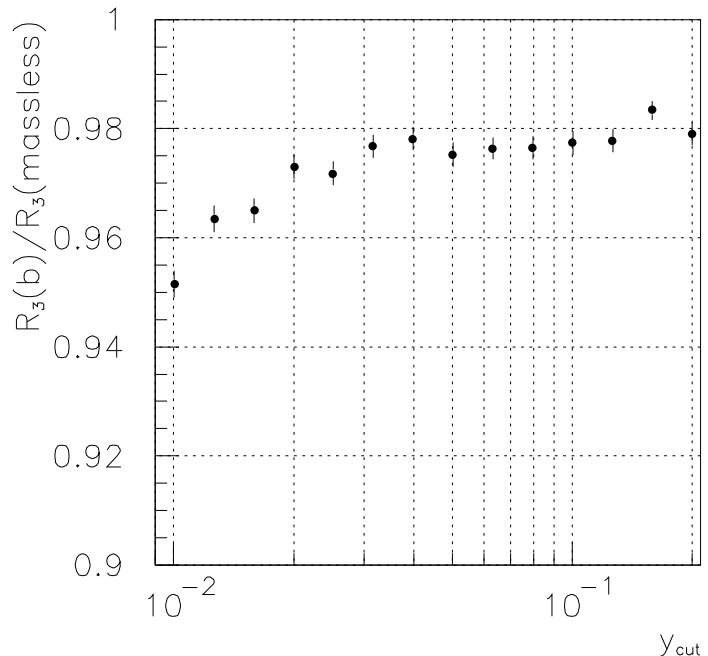

Figure 5.20: The numerical calculation of the ratio; $R_{3}^{b} / R_{3}^{0}$ for the Durham algorithm as a function of $y_{\text {cut }}$ using $m_{b}\left(M_{Z}\right)=3.0 \mathrm{GeV}$ [121]. The plots and error bars represent the central values of the numerical calculation and its errors.

For illustration, the measured ratios $R_{3}^{i} / R_{3}^{\text {all }}(i=u d s, c, b)$ are shown in Fig. 5.22(a). In this case of $b$-quarks, $R_{3}^{b} / R_{3}^{\text {all }}$ lies above unity for the JADE family of algorithms E, E0, P and P0, and below unity for the D and G algorithms; note that all six data points are highly correlated with each other, so that the differences between algorithms are more significant than naively implied by the statistical errors displayed. For comparison, the corresponding QCD calculations of $R_{3}^{b} / R_{3}^{0}$ [121] are shown in Fig. 5.22(a) for an input value of $\alpha_{s}\left(M_{Z}^{2}\right)=0.118$ under the assumption of a flavor-independent strong coupling. Under this assumption the calculations are in good agreement with the data, and the data clearly demonstrate the effects of the non-zero $b$-quark mass, which are up to twice as large as the statistical error. 
For $c$-quarks mass effects are expected to be $\mathcal{O}(1 \%)$ or less [121], which is much smaller than our statistical error of roughly $4 \%$ on $R_{3}^{c} / R_{3}^{\text {all }}$. This effects of non-zero $c$-quark mass, and of the light-quark masses, will hence be neglected here.

Table 5.12: Massless coefficients $A^{0}, B^{0}$, and $C^{0}$ for each algorithm

\begin{tabular}{|l|r||r|r|r|}
\hline Algorithm & $y_{c}$ & $A^{0}$ & $B^{0}$ & $C^{0}$ \\
\hline E & 0.040 & 14.21 & 402.48 & 26.0 \\
\hline E0 & 0.020 & 24.35 & 237.1 & 116.6 \\
\hline P & 0.020 & 24.35 & 72.9 & 116.6 \\
\hline P0 & 0.015 & 29.36 & 14.55 & 187.6 \\
\hline D & 0.010 & 15.71 & 122.88 & 83.98 \\
\hline G & 0.080 & 11.61 & 46.45 & 64.76 \\
\hline
\end{tabular}

Table 5.13: The coefficient $A^{b}, B^{b}, C^{b}$ for the massive next-to-leading-order calculation. The numbers in parentheses represent the estimated numerical precision.

\begin{tabular}{|l|r||r|r|r||r|r|r|}
\hline & \multicolumn{1}{|c||}{} & \multicolumn{3}{c||}{$A^{b}$ for $m_{b}\left(M_{Z}\right)\left(\mathrm{GeV} / c^{2}\right)=$} & \multicolumn{3}{c|}{$B^{b}$ for $m_{b}\left(M_{Z}\right)\left(\mathrm{GeV} / c^{2}\right)=$} \\
\hline Algorithm & $y_{c}$ & 2.5 & 3 & 3.5 & 2.5 & 3 & 3.5 \\
\hline E & 0.040 & $14.392(1)$ & $14.459(1)$ & $14.543(1)$ & $443(4)$ & $466(4)$ & $487(4)$ \\
\hline E0 & 0.020 & $24.850(2)$ & $25.024(2)$ & $25.231(2)$ & $277(4)$ & $291(4)$ & $310(4)$ \\
\hline P & 0.020 & $24.850(2)$ & $25.024(2)$ & $25.231(2)$ & $63(4)$ & $67(4)$ & $75(4)$ \\
\hline P0 & 0.015 & $30.054(2)$ & $30.315(2)$ & $30.631(2)$ & $2(4)$ & $14(4)$ & $29(4)$ \\
\hline D & 0.010 & $15.355(2)$ & $15.213(2)$ & $15.060(2)$ & $105(4)$ & $102(4)$ & $99(4)$ \\
\hline G & 0.080 & $11.493(1)$ & $11.435(1)$ & $11.365(1)$ & $61(4)$ & $58(4)$ & $57(4)$ \\
\hline
\end{tabular}

\begin{tabular}{|l|r||r|r|r|}
\hline \multicolumn{1}{|c|}{} & \multicolumn{1}{|c||}{} & \multicolumn{3}{c|}{$C^{b}$ for $m_{b}\left(M_{Z}\right)\left(\mathrm{GeV} / c^{2}\right)=$} \\
\hline Algorithm & $y_{c}$ & 2.5 & 3 & 3.5 \\
\hline E & 0.040 & $27.91(1)$ & $28.27(1)$ & $28.71(1)$ \\
\hline E0 & 0.020 & $125.39(7)$ & $127.34(7)$ & $129.55(8)$ \\
\hline P & 0.020 & $125.39(7)$ & $127.34(7)$ & $129.55(8)$ \\
\hline P0 & 0.015 & $202.8(1)$ & $206.1(1)$ & $209.4(1)$ \\
\hline D & 0.010 & $84.30(6)$ & $82.83(6)$ & $81.19(6)$ \\
\hline G & 0.080 & $65.55(4)$ & $64.60(3)$ & $63.56(3)$ \\
\hline
\end{tabular}

$$
\begin{aligned}
\frac{R_{3}^{i}}{R_{3}^{\text {all }}} & =\frac{A^{i}\left(y_{\text {cut }}\right) \bar{\alpha}_{s}^{i}+\left[B^{i}\left(y_{\text {cut }}\right)+C^{i}\left(y_{\text {cut }}\right)\right]\left(\bar{\alpha}_{s}^{i}\right)^{2}}{A^{0}\left(y_{\text {cut }}\right) \bar{\alpha}_{s}^{\text {all }}+\left[B^{0}\left(y_{\text {cut }}\right)+C^{0}\left(y_{\text {cut }}\right)\right]\left(\bar{\alpha}_{s}^{\text {all }}\right)^{2}} \\
& =\frac{A^{i} r_{i}+\alpha_{s}^{\text {all }} B^{i} r_{i}{ }^{2}}{A^{0}+\alpha_{s}^{\text {all }} B^{0}}
\end{aligned}
$$

where we use the notation $r_{i}=\alpha_{s}^{i} / \alpha_{s}^{\text {all }}$, and the world-average value of $\alpha_{s}^{\text {all }}\left(M_{Z}^{2}\right)=0.118[32$ were $u^{2} d^{2}$. Note that the partial derivative matrix for the error propagation is diagonal:

$$
\begin{aligned}
S_{i i} & =\frac{\partial r_{i}}{\partial R^{i}} \simeq 1 / \frac{\partial R^{i}}{\partial r_{i}} \\
& =\frac{A^{0}+\bar{\alpha}_{s}^{\text {all }}\left(B^{0}+C^{0}\right)}{A^{i}+2 \bar{\alpha}_{s}^{\text {all }}\left(B^{i}+C^{i}\right) r_{i}} \\
S_{i j} & =\frac{\partial r_{i}}{\partial R^{j}}=0 . \quad(i \neq j)
\end{aligned}
$$

where $R^{i}=R_{3}^{i} / R_{3}^{\text {all }}$. For example, for the E0 algorithm and $y_{\text {cut }}=0.02$ the statistical error matrix for $r_{i}$ was calculated:

$$
V(r)=S V(R) S^{T}=\left(\begin{array}{rrr}
0.0000605 & -0.0001969 & -0.0000108 \\
-0.0001969 & 0.0008728 & -0.0001387 \\
-0.0000108 & -0.0001387 & 0.0001329
\end{array}\right)
$$

and the statistical errors on $r_{i}$ were found to be $\sigma\left(r_{i}\right)=\sqrt{V_{i i}}=(0.008,0.030,0.012)$.

The $\alpha_{s}^{i} / \alpha_{s}^{a l l}$ values and their statistical errors are listed in Table. 5.18, and also shown in Fig. 5.22(b). The experimental systematic errors and the hadronization uncertainties were evaluated through Eqn. 5.25, and are also summarized in Table. 5.18. It can be seen that

${ }^{2}$ It turned out that the equations of the naive translation taking into account the flavor fractions in the denominator:

$$
\frac{R_{3}^{i}\left(y_{\text {cut }}\right)}{R_{3}^{\text {all }}\left(y_{\text {cut }}\right)}=\frac{A^{i}\left(y_{\text {cut }}\right) \bar{\alpha}_{s}^{i}+\left[B^{i}\left(y_{\text {cut }}\right)+C^{i}\left(y_{\text {cut }}\right)\right]\left(\bar{\alpha}_{s}^{i}\right)^{2}}{\sum_{j} f_{j}\left(A^{j}\left(y_{\text {cut }}\right) \bar{\alpha}_{s}^{j}+\left[B^{j}\left(y_{\text {cut }}\right)+C^{j}\left(y_{\text {cut }}\right)\right]\left(\bar{\alpha}_{s}^{j}\right)^{2}\right)}
$$

are parallel each other because one degree of freedom is lost by taking ratios. However, we evaluate 3 ratios $\alpha_{s}^{u d s} / \alpha_{s}^{a l l}, \alpha_{s}^{c} / \alpha_{s}^{\text {all }}$ and $\alpha_{s}^{b} / \alpha_{s}^{\text {all }}$ by substantially similar manner with our previous publication [9] as a comparison. 
the $\alpha_{s}^{i} / \alpha_{s}^{\text {all }}$ ratios determined using the different jet algorithms are consistent with unity, and are in good agreement with one another. Note that the deviations between algorithms on $R_{3}^{b} / R_{3}^{a l l}$ are significantly reduced through the translation to $\alpha_{s}^{b} / \alpha_{s}^{\text {all }}$ using the $\mathcal{O}\left(\alpha_{s}^{2}\right)$ calculations taking into account $b$ mass effects.

\section{Correlation between flavors}

Since the flavor tagged subsample could not be completely pure, there are some correlations between the unfolded results. The correlation coefficients $\rho(k, l)$ between flavors $(k-l)$ are evaluated from the full covariance matrix $V$ :

$$
\rho(k, l)=\frac{V_{k l}}{\sqrt{V_{k k} V_{l l}}}
$$

$\rho(k, l)$ could vary as $-1 \leq \rho(k, l) \leq 1$, and $\rho(k, l)=0$ means no correlation between $k$ and l. Table 5.14 shows the correlations between flavors on $R_{3}^{i}, R_{3}^{i} / R_{3}^{\text {all }}$, and $\alpha_{s}^{i} / \alpha_{s}^{\text {all }}$.

Because of the high purity ( $>90 \%$ ) of the $u d s$ and $b$ tagged subsamples, the correlations on $R_{3}$ between these two samples are small. The correlations on $R_{3}$ between $c$ and the other flavors are relatively large due to the lower purity $(\sim 65 \%)$ of the charm tagged subsample, however, substantially improved from the lifetime based tagging $(\rho(u d s, c)=$ $-0.76, \rho(u d s, b)=0.30, \rho(c, b)=-0.55$ [9], see also Sect. 5.6).

The correlations on ratios are quite large especially between $u d s$ and $c$. Unfortunately, this is inevitable because those are the ratios of small samples ( $c$ and $b)$ to all dominated by $u d s$, although the flavor tagging is optimized to minimize the flavor correlations on the jet rate $R_{3}^{i}$.

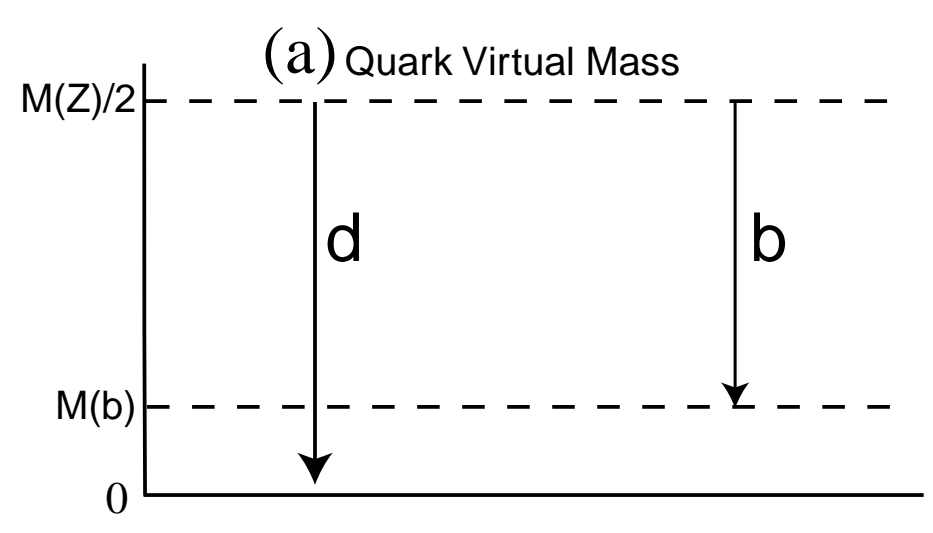

\section{Jet-clustering Invariant Mass}

(b)

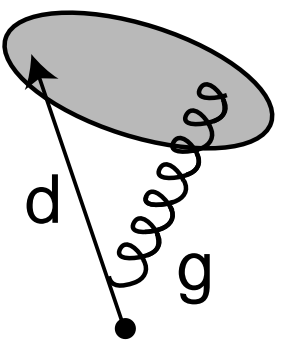

(c)

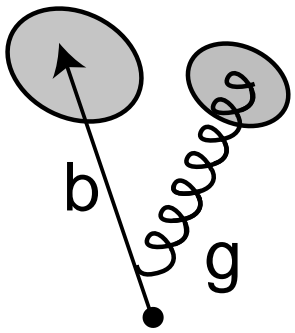

$$
\mathrm{m}_{\mathrm{ij}}^{2}>\mathrm{y}_{\text {cut }} \mathrm{S}
$$

Figure 5.21: Schematic view of the modification of jet rate for $b$ event. (a) Phase space of quark virtual mass in parton shower evolution. (b-c) Invariant mass and jet resolution parameter $y_{c u t}$. 

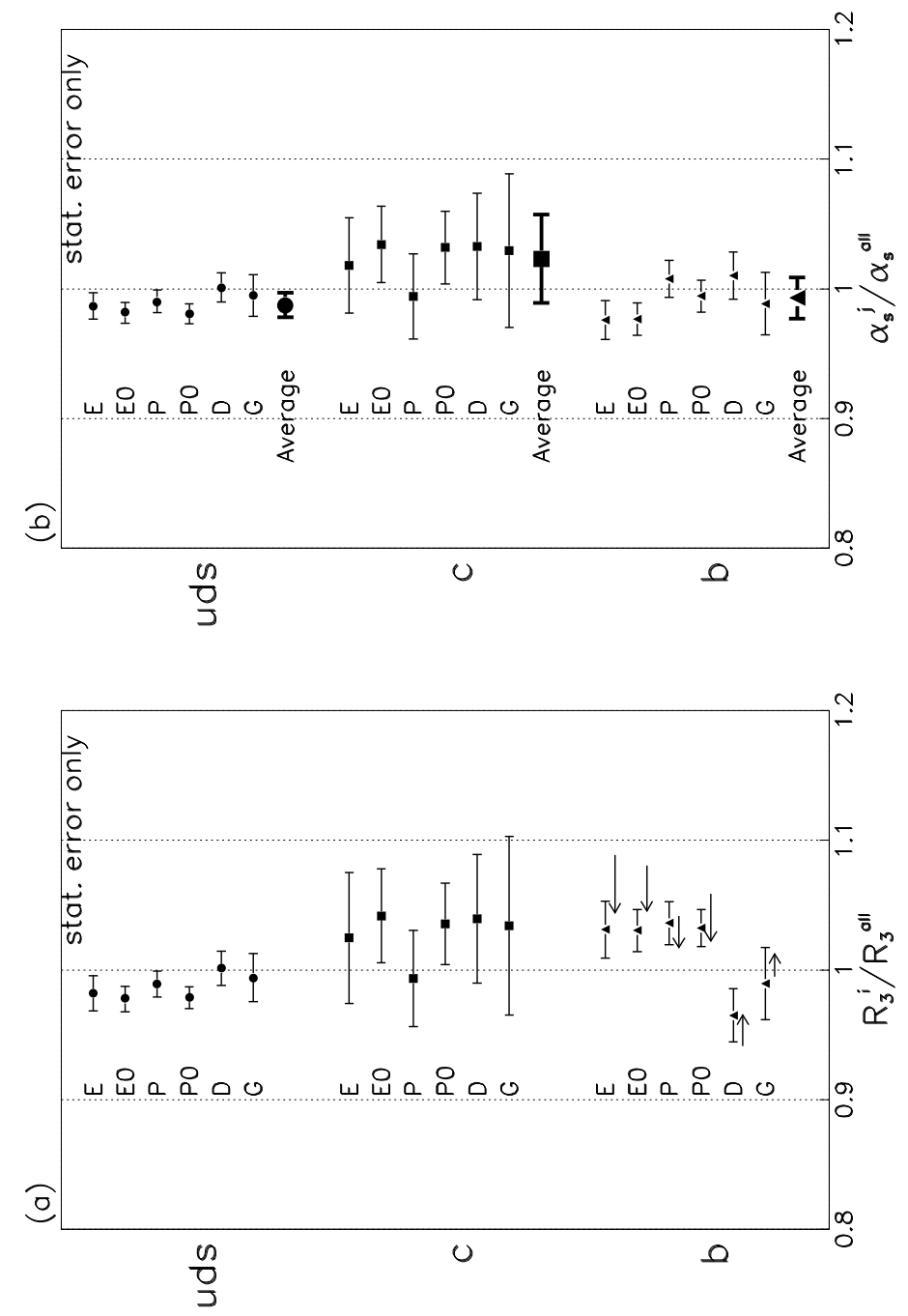

Figure 5.22: The measured ratios $R_{3}^{i} / R_{3}^{\text {all }}$ (a), and the corresponding translated ratios $\alpha_{s}^{i} / \alpha_{s}^{\text {all }}$ $(i=u d s, c, b)(\mathrm{b})$. The arrows in (a) indicate the range of the theoretical prediction described in the text for values of the $b$-quark mass in the range $2.5 \leq m_{b}\left(M_{Z}\right) \leq 3.5 \mathrm{GeV} / c^{2}$, with the arrow pointing towards the lower mass value. In (b) the weighted average over the six algorithms is also shown. In all cases only statistical error bars are displayed.

\begin{tabular}{|c|c|c|c|c|c|c|}
\hline & uds-c & uds-b & $c-b$ & uds-c & uds-b & $c-b$ \\
\hline & \multicolumn{3}{|c|}{$\mathrm{E}$} & \multicolumn{3}{|c|}{ E0 } \\
\hline$R_{3}^{i}$ & -0.40 & 0.04 & -0.18 & 0.39 & 0.04 & -0.19 \\
\hline$R_{3}^{\prime} R_{3}^{\text {all }}$ & -0.87 & -0.10 & -0.41 & -0.86 & -0.12 & -0.41 \\
\hline \multirow[t]{2}{*}{$\alpha_{s}^{i} / \alpha_{s}^{\text {all }}$} & -0.87 & -0.10 & -0.41 & -0.86 & -0.12 & -0.41 \\
\hline & \multicolumn{3}{|c|}{$\mathrm{P}$} & \multicolumn{3}{|c|}{ P0 } \\
\hline$R_{3}^{i}$ & -0.39 & 0.04 & 0.19 & -0.38 & 0.04 & -0.19 \\
\hline$R_{3}^{\prime} R_{3}^{\text {all }}$ & -0.86 & -0.12 & -0.41 & -0.85 & -0.13 & -0.41 \\
\hline \multirow[t]{2}{*}{$\alpha_{s}^{i} / \alpha_{s}^{\text {all }}$} & -0.86 & -0.12 & -0.41 & -0.85 & -0.13 & -0.41 \\
\hline & \multicolumn{3}{|c|}{$\mathrm{D}$} & \multicolumn{3}{|c|}{ G } \\
\hline$R_{3}^{i}$ & -0.42 & 0.04 & -0.19 & -0.48 & 0.05 & -0.19 \\
\hline$R_{3}^{\prime} R_{3}^{\text {all }}$ & -0.88 & -0.07 & -0.41 & -0.89 & -0.05 & -0.41 \\
\hline$\alpha_{s}^{i} / \alpha_{s}^{\text {all }}$ & -0.88 & -0.07 & -0.41 & -0.89 & -0.05 & -0.41 \\
\hline
\end{tabular}

\subsubsection{Translation Uncertainties}

In addition to the hadronization uncertainty, theoretical uncertainties arise from the translation process between the measured jet-rate ratios $R_{3}^{i} / R_{3}^{\text {all }}$ and the corresponding $\alpha_{s}$ ratios $\alpha_{s}^{i} / \alpha_{s}^{\text {all }}$. From an operational point of view the sources of uncertainty affect the values of the coefficients $A^{i}, B^{i}$ and $C^{i}$ used for the translation. For each variation considered the relevant $A^{i}, B^{i}$ or $C^{i}$ were reevaluated, the ratios $\alpha_{s}^{i} / \alpha_{s}^{\text {all }}$ were rederived, and the derivation w.r.t the central value was assigned as a systematic uncertainty.

We considered:

- Heavy Quark Mass

We used a central value of the running $b$-quark mass $m_{b}\left(M_{Z}\right)=3.0 \mathrm{GeV} / c^{2}$ and a variation of $\pm 0.5 \mathrm{GeV} / c^{2}$. This corresponds to the range $3.62<m_{b}\left(m_{b}\right)<5.06 \mathrm{GeV} / c^{2}$ and covers generously values determined from the $\Upsilon$ system using QCD sum rules, $4.13 \pm 0.06 \mathrm{GeV} / c^{2}$, as well as using lattice QCD, $4.15 \pm 0.20 \mathrm{GeV} / c^{2}[127,129]$. It is also consistent with the recent DELPHI measurement of the running mass: $m_{b}\left(M_{Z}\right)=$ $2.67 \pm 0.25$ (stat.) \pm 0.34 (frag.) \pm 0.27 (theo.) $\mathrm{GeV} / c^{2}[71]$. 
- Numerical Accuracy

The calculations of the coefficients $A^{i}, B^{i}$ and $C^{i}$ in both the massless and massive cases are performed using Monte Carlo integrations over phase-space, and the numerical accuracy achieved hence depends on the length of time for which the computer programs were run. The resulting statistical errors on the coefficients $A^{i}, B^{i}$ and $C^{i}$ are in all cases negligibly small on the scale of the experimental statistical errors on $\alpha_{s}^{i} / \alpha_{s}^{\text {all }}$.

- Higher-order Perturbative QCD Contributions

Eq. 5.24 is formally accurate only up to $\mathcal{O}\left(\alpha_{s}^{2}\right)$, and a priori unknown $\mathcal{O}\left(\alpha_{s}^{3}\right)$ and higher order terms will contribute. In the ratios $R_{3}^{i} / R_{3}^{\text {all }}$ the effects of such higherorder contributions will tend to cancel. Nevertheless we have attempted to evaluate the residual uncertainty due to missing higher-order contributions.

For this purpose a standard procedure is to vary the QCD renormalization scale $\mu$; see eg.[31]. Operationally such a variation modifies the NLO 3-jet term $B^{i}$ in Eq. 5.25 :

$$
B^{i} \rightarrow B^{i}-2 \pi b_{0} \ln f \times A^{i}
$$

where $f=\mu^{2} / s, b_{0}=\left(33-2 n_{f}\right) /(12 \pi)$, and $n_{f}$ is the number of active quark flavors $n_{f}=5$ at $\sqrt{s}=M_{Z}$. Following our study of $\alpha_{s}^{\text {all }}$ [31], for each jet algorithm we varied $f$ and $\alpha_{s}^{\text {all }}$ simultaneously in the range allowed by fits to the flavor-inclusive differential 2-jet rate. $f$-range was defined such that the goodness of the fit $\chi_{\text {d.o.f }}^{2}<5$ and $f<4.0$ (Fig. 5.23). The former requirement excludes the low $f$ regions where the fit quality is poor. The latter requirement corresponds to a reasonable physical limit $\mu \leq 2 \sqrt{s}$. We evaluated the ratios $\alpha_{s}^{i} / \alpha_{s}^{\text {all }}$ for each algorithm at $\left(f_{0}, \alpha_{s}\right),\left(f_{+}, \alpha_{s}+\Delta \alpha_{s}\right)$, and $\left(f_{-}, \alpha_{s}+\Delta \alpha_{s}\right)$. and assigned the variation w.r.t the central value as theoretical uncertainties due to the renormalization scale and overall $\alpha_{s}^{a l l}$. The corresponding central $\alpha_{s}$ and $f$ values and respective variations are listed in Table 5.16. We neglected any explicit mass-dependence in the renormalization-scale dependence of $B^{i}$.

In addition, we considered NLO contribution to the 4-jet rate. These enter formally at $\mathcal{O}\left(\alpha_{s}^{3}\right)$ in Eq. 5.24, although operationally they may be estimated by variation of the LO coefficient $C^{i}$. Since the 4 -jet rate has been calculated recently completed at NLO for massless quarks [130], these terms can be estimated reliably. For our jet algorithms and $y_{\text {cut }}$ values Dixon has evaluated the LO and NLO 4-jet contributions [131]; these are listed in Table 5.15. Based on these calculations we varied the $C^{i}$ by $\pm 100 \%$. For each jet algorithm, at the chosen $y_{\text {cut }}$ value, the measured contribution to $R_{3}^{i}$ from $\geq 5$ jet states was smaller than $1 \%$ and the corresponding $\mathcal{O}\left(\alpha_{s}^{3}\right)$ contributions to Eqn. 5.25 were neglected.

These uncertainties are listed in Table 5.17. For each jet algorithm and flavor, the uncertainties were added in quadrature to define a translation uncertainty on $\alpha_{s}^{i} / \alpha_{s}^{\text {all }}$; these are listed in Table 5.17 and 5.18. The translation uncertainty on $\alpha_{s}^{b} / \alpha_{s}^{\text {all }}$ is comparable in magnitude with the statistical error.

Table 5.15: Leading and next-to-leading order contributions to the 4-jet rate. Note that no results are given for the $\mathrm{P} 0$ algorithm; instead results are shown for the $\mathrm{P}$ algorithm at the $\mathrm{P} 0 y_{c}$ value of 0.015 . Since the $\mathrm{P}$ and $\mathrm{P} 0$ algorithms are identical at leading order, one expects the next-to-leading order contributions to be similar also for these two cases.

\begin{tabular}{|c|l|c|c|}
\hline Algorithm & $y_{c}$ & LO contribution & NLO contribution \\
\hline E & 0.04 & 0.00918 & 0.0210 \\
E0 & 0.02 & 0.0413 & 0.0720 \\
P & 0.02 & 0.0413 & 0.0637 \\
P & 0.015 & 0.0665 & 0.1005 \\
D & 0.01 & 0.0298 & 0.0470 \\
G & 0.08 & 0.0226 & 0.0325 \\
\hline
\end{tabular}


Table 5.16: Renormalization scale and corresponding $\alpha_{s}$ ranges used to estimate higherorder perturbative QCD uncertainties. $f_{+}, f_{-}$and $\alpha_{s}$ values for each algorithm are taken from Table VIII and $f_{0}$ values are read from Fig. 5.23 (copied from Fig.32 in [31]).

\begin{tabular}{|c|cc|cc|cc|}
\hline & $f_{-}$ & $\Delta \alpha_{s}$ & $f_{0}$ & $\alpha_{s}$ & $f_{+}$ & $\Delta \alpha_{s}$ \\
\hline E & $5 \times 10^{-5}$ & -0.0217 & 0.2 & 0.1273 & 4. & +0.0217 \\
E0 & $1.2 \times 10^{-2}$ & -0.0083 & 0.7 & 0.1175 & 4. & +0.0083 \\
P & $5.5 \times 10^{-3}$ & -0.0053 & 0.5 & 0.1207 & 4. & +0.0053 \\
P0 & $1.2 \times 10^{-2}$ & -0.0057 & 0.7 & 0.1190 & 4. & +0.0057 \\
D & $1.7 \times 10^{-3}$ & -0.0077 & 1.0 & 0.1245 & 4. & +0.0077 \\
G & $4 \times 10^{-3}$ & -0.0043 & 1.0 & 0.1191 & 4. & +0.0043 \\
\hline
\end{tabular}

Figure 5.23: (a) $\alpha_{s}\left(M_{Z}^{2}\right)$ and (b) $\chi_{\text {dof }}^{2}$ from the $\mathcal{O}\left(\alpha_{s}^{2}\right)$ fits to the jet rates as a function of renormalization scale factor $f$ (see text).

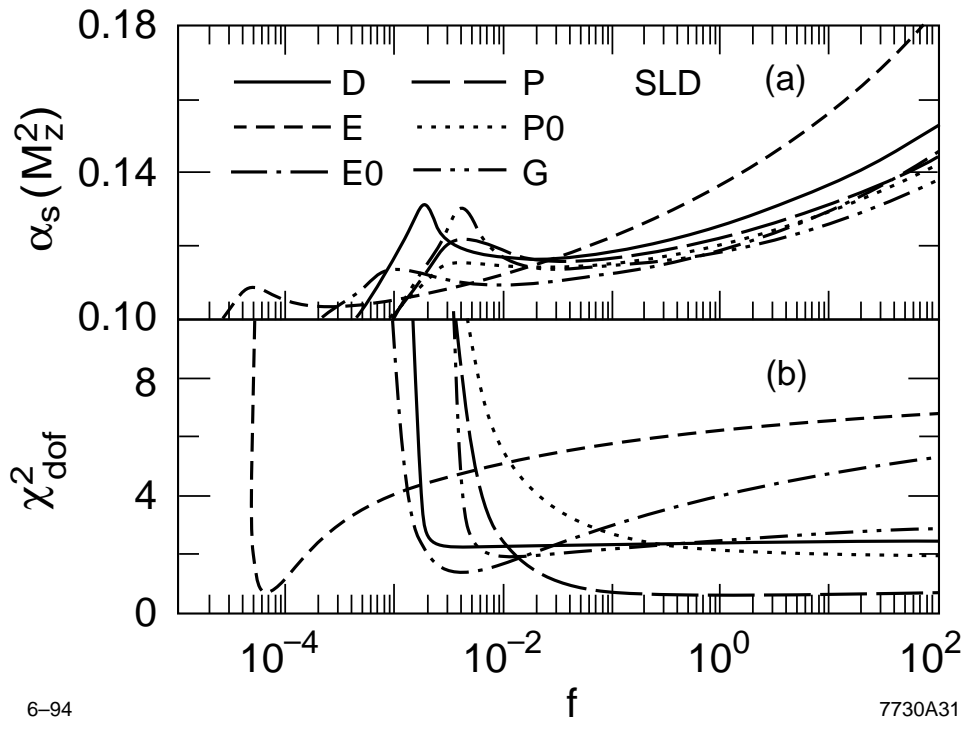

Table 5.17: Summary of translation uncertainties on the $\alpha_{s}$ ratios for each algorithm; '+' ('-') denotes the error corresponding to the relevant positive (negative) parameter variation.

\begin{tabular}{|c|c|c|c|c|c|c|c|c|}
\hline \multirow[t]{2}{*}{ source } & \multirow[t]{2}{*}{ Center } & \multirow[t]{2}{*}{ Variation } & \multicolumn{2}{|c|}{$\delta \alpha_{s}^{u d s} / \alpha_{s}^{\text {all }}(\%)$} & \multicolumn{2}{|c|}{$\delta \alpha_{s}^{c} / \alpha_{s}^{\text {all }}(\%)$} & \multicolumn{2}{|c|}{$\delta \alpha_{s}^{b} / \alpha_{s}^{a l l}(\%)$} \\
\hline & & & + & 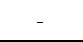 & + & - & + & - \\
\hline & \multicolumn{8}{|c|}{ E-algo $\left(y_{c}=0.04\right)$} \\
\hline$m_{b}\left(M_{Z}\right)$ & $3.0 \mathrm{GeV}$ & \pm 0.5 & 0. & 0. & 0. & 0. & -1.40 & 1.52 \\
\hline$\mu, \alpha_{s}$ dep. & & & -0.049 & 0.090 & 0.068 & -0.124 & -0.628 & 0.715 \\
\hline$\geq 4$ jet contrib. & C & $\pm C$ & 0.013 & -0.014 & -0.018 & 0.019 & -0.042 & 0.045 \\
\hline \multirow[t]{2}{*}{ Total } & & & 0.091 & -0.051 & 0.070 & -0.126 & 1.68 & -1.53 \\
\hline & \multicolumn{8}{|c|}{ E0-algo $\left(y_{c}=0.02\right)$} \\
\hline$m_{b}\left(M_{Z}\right)$ & $3.0 \mathrm{GeV}$ & \pm 0.5 & 0. & 0. & 0 . & 0 . & -1.41 & 1.14 \\
\hline$\mu, \alpha_{s}$ dep. & & & -0.115 & 0.167 & 0.220 & -0.316 & -0.559 & 0.677 \\
\hline$\geq 4$ jet contrib. & $C$ & $\pm C$ & 0.075 & -0.095 & -0.143 & 0.181 & -0.055 & 0.070 \\
\hline \multirow[t]{2}{*}{ Total } & & & 0.183 & -0.149 & 0.285 & -0.347 & 1.34 & -1.52 \\
\hline & \multicolumn{8}{|c|}{ P-algo $\left(y_{c}=0.02\right)$} \\
\hline$m_{b}\left(M_{Z}\right)$ & $3.0 \mathrm{GeV}$ & \pm 0.5 & 0. & 0. & 0. & 0 . & -1.22 & 0.902 \\
\hline$\mu, \alpha_{s}$ dep. & & & -0.106 & 0.120 & -0.064 & 0.073 & 0.089 & -0.099 \\
\hline$\geq 4$ jet contrib. & C & $\pm C$ & 0.051 & -0.067 & 0.031 & -0.041 & -0.427 & 0.576 \\
\hline \multirow[t]{2}{*}{ Total } & & & 0.130 & -0.126 & 0.079 & -0.076 & 1.07 & -1.30 \\
\hline & \multicolumn{8}{|c|}{$\mathrm{P} 0$-algo $\left(y_{c}=0.015\right)$} \\
\hline$m_{b}\left(M_{Z}\right)$ & $3.0 \mathrm{GeV}$ & \pm 0.5 & 0. & 0. & 0. & 0 . & -1.66 & 1.44 \\
\hline$\mu, \alpha_{s}$ dep. & & & -0.189 & 0.238 & 0.328 & -0.405 & -0.157 & 0.176 \\
\hline$\geq 4$ jet contrib. & C & $\pm C$ & 0.135 & -0.197 & -0.231 & 0.343 & -0.410 & 0.615 \\
\hline \multirow[t]{2}{*}{ Total } & & & 0.273 & -0.273 & 0.474 & -0.466 & 1.58 & -1.72 \\
\hline & \multicolumn{8}{|c|}{ D-algo $\left(y_{c}=0.010\right)$} \\
\hline$m_{b}\left(M_{Z}\right)$ & $3.0 \mathrm{GeV}$ & \pm 0.5 & 0. & 0. & 0. & 0. & 1.11 & -1.01 \\
\hline$\mu, \alpha_{s}$ dep. & & & 0.006 & -0.015 & 0.190 & -0.478 & 0.283 & -0.513 \\
\hline$\geq 4$ jet contrib. & C & $\pm C$ & -0.005 & 0.007 & -0.158 & 0.209 & -0.257 & 0.342 \\
\hline \multirow[t]{2}{*}{ Total } & & & 0.009 & -0.016 & 0.282 & -0.503 & 1.20 & -1.17 \\
\hline & \multicolumn{8}{|c|}{ G-algo $\left(y_{c}=0.08\right)$} \\
\hline$m_{b}\left(M_{Z}\right)$ & $3.0 \mathrm{GeV}$ & \pm 0.5 & 0. & 0. & 0. & 0. & 0.67 & -0.80 \\
\hline$\mu, \alpha_{s}$ dep. & & & -0.036 & 0.077 & 0.213 & -0.452 & -0.286 & 0.522 \\
\hline$\geq 4$ jet contrib. & C & $\pm C$ & 0.029 & -0.039 & -0.169 & 0.233 & 0.097 & -0.131 \\
\hline Total & & & 0.082 & -0.053 & 0.316 & -0.482 & 0.86 & -0.86 \\
\hline
\end{tabular}




\subsubsection{Comparison of $\alpha_{s}$ Ratios}

For each jet algorithm $n$, the statistical and experimental systematic errors were added in quadrature with the hadronization and translation uncertainties (Table 5.18) to define a total error $\sigma_{n}^{i}$ on $\alpha_{s}^{i} / \alpha_{s}^{\text {all }}(i=u d s, c, b)$. For each flavor a single value of $\alpha_{s}^{i} / \alpha_{s}^{\text {all }}$ was then defined by taking the weighted average of the results over the six jet algorithms:

$$
\alpha_{s}^{i} / \alpha_{s}^{\text {all }}=\sum_{n} w_{n}^{i}\left(\alpha_{s}^{i} / \alpha_{s}^{a l l}\right)_{n}
$$

where $w_{n}^{i}$ is the weight for each algorithm:

$$
w_{n}^{i}=\frac{1 / \sigma_{n}^{i 2}}{\sum_{m} 1 / \sigma_{m}^{i 2}}
$$

The average statistical and experimental systematic errors were each computed from:

$$
\overline{\sigma^{i}}=\sqrt{\sum_{n m} E_{n m}^{i} w_{n}^{i} w_{m}^{i}}
$$

where $E^{i}$ is the $6 \times 6$ covariant matrix with elements:

$$
E_{n m}^{i}=\sigma_{m}^{i} \sigma_{n}^{i}
$$

and $100 \%$ correlation was conservatively assumed among algorithms. The average translation and hadronization uncertainties were calculated in a similar fashion. We then calculated the r.m.s. deviation on $\alpha_{s}^{i} / \alpha_{s}^{\text {all }}$, shown in Table 5.18, and assigned this scatter between the results from different algorithms as an additional theoretical uncertainty, since all of these algorithms were applied to the same data set. The average translation and hadronization uncertainties were added in quadrature together with the r.m.s. deviation to define the total theoretical uncertainty.

We obtained:

$$
\begin{aligned}
\alpha_{s}^{\text {uds }} / \alpha_{s}^{\text {all }} & =0.987 \pm 0.010(\text { stat } .)_{-0.010}^{+0.012}(\text { syst. })_{-0.008}^{+0.009}(\text { theory }) \\
\alpha_{s}^{c} / \alpha_{s}^{\text {all }} & =1.023 \pm 0.034(\text { stat } .)_{-0.036}^{+0.032}(\text { syst } .)_{-0.014}^{+0.018}(\text { theory }) \\
\alpha_{s}^{b} / \alpha_{s}^{\text {all }} & =0.993 \pm 0.016(\text { stat } .)_{-0.023}^{+0.020}(\text { syst } .)_{-0.027}^{+0.019}(\text { theory })
\end{aligned}
$$

These ratios are shown in Fig. 5.22(b). The theoretical uncertainties are only slightly smalle than the respective experimental systematic errors, and comprise roughly equal contributions from the hadronization and translation uncertainties, as well as from the r.m.s. deviation over the six jet algorithms.

Table 5.18: Central values of $\alpha_{s}^{i} / \alpha_{s}^{\text {all }}$ and errors.

\begin{tabular}{|l|c|c|c|c|c|c|c|}
\hline Algorithm & $\mathrm{E}$ & $\mathrm{E} 0$ & $\mathrm{P}$ & $\mathrm{P} 0$ & $\mathrm{D}$ & $\mathrm{G}$ & \\
$y_{c}$ & 0.040 & 0.020 & 0.020 & 0.015 & 0.010 & 0.080 & \\
\hline \multicolumn{7}{|c|}{$\alpha_{s}^{\text {uds }} / \alpha_{s}^{\text {all }}$} \\
\hline central val. & 0.987 & 0.981 & 0.990 & 0.981 & 1.001 & 0.995 & 0.0074 \\
\hline stat. & 0.010 & 0.008 & 0.009 & 0.008 & 0.011 & 0.016 & \\
\hline exp. syst. & ${ }_{-0.011}^{+0.012}$ & ${ }_{-0.009}^{+0.009}$ & ${ }_{-0.010}^{+0.010}$ & ${ }_{-0.009}^{+0.009}$ & ${ }_{-0.013}^{+0.013}$ & ${ }_{-0.015}^{+0.016}$ & \\
\hline hadronisation & ${ }_{-0.002}^{+0.007}$ & ${ }_{-0.002}^{+0.006}$ & ${ }_{-0.001}^{+0.005}$ & ${ }_{-0.002}^{+0.005}$ & ${ }_{-0.001}^{+0.001}$ & ${ }_{-0.004}^{+0.002}$ & \\
\hline translation & ${ }_{-0.0005}^{+0.0009}$ & ${ }_{-0.0015}^{+0.0018}$ & ${ }_{-0.0013}^{+0.0013}$ & ${ }_{-0.0027}^{+0.0027}$ & ${ }_{-0.0002}^{+0.0001}$ & ${ }_{-0.0005}^{+0.0008}$ & \\
\hline \multicolumn{7}{|c|}{$\alpha_{s}^{c} / \alpha_{s}^{\text {all }}$} \\
\hline central val. & 1.018 & 1.034 & 0.994 & 1.032 & 1.033 & 1.029 & 0.014 \\
\hline stat. & 0.037 & 0.030 & 0.033 & 0.028 & 0.041 & 0.059 & \\
\hline exp. syst. & ${ }_{-0.045}^{+0.035}$ & ${ }_{-0.031}^{+0.028}$ & ${ }_{-0.034}^{+0.031}$ & ${ }_{-0.029}^{+0.026}$ & ${ }_{-0.045}^{+0.040}$ & ${ }_{-0.059}^{+0.049}$ & \\
\hline hadronisation & ${ }_{-0.000}^{+0.012}$ & ${ }_{-0.001}^{+0.015}$ & ${ }_{-0.001}^{+0.012}$ & ${ }_{-0.000}^{+0.015}$ & ${ }_{-0.002}^{+0.005}$ & ${ }_{-0.003}^{+0.004}$ & \\
\hline translation & ${ }_{-0.0013}^{+0.0007}$ & ${ }_{-0.0035}^{+0.0029}$ & ${ }_{-0.0008}^{+0.008}$ & ${ }_{-0.0047}^{+0.0047}$ & ${ }_{-0.0050}^{+0.0028}$ & ${ }_{-0.0048}^{+0.0032}$ & \\
\hline \multicolumn{7}{|c|}{$\alpha_{s}^{b} / \alpha_{s}^{\text {all }}$} \\
\hline central val. & 0.976 & 0.977 & 1.008 & 0.994 & 1.010 & 0.989 & 0.013 \\
\hline stat. & 0.015 & 0.012 & 0.014 & 0.012 & 0.018 & 0.024 & \\
\hline exp. & ${ }_{-0.022}^{+0.021}$ & ${ }_{-0.021}^{+0.017}$ & ${ }_{-0.023}^{+0.018}$ & ${ }_{-0.022}^{+0.018}$ & ${ }_{-0.027}^{+0.027}$ & ${ }_{-0.023}^{+0.022}$ & \\
\hline hadronisation & ${ }_{-0.026}^{+0.006}$ & ${ }_{-0.028}^{+0.003}$ & ${ }_{-0.019}^{+0.000}$ & ${ }_{-0.026}^{+0.003}$ & ${ }_{-0.005}^{+0.000}$ & ${ }_{-0.005}^{+0.013}$ & \\
\hline translation & ${ }_{-0.015}^{+0.017}$ & ${ }_{-0.015}^{+0.013}$ & ${ }_{-0.013}^{+0.011}$ & ${ }_{-0.017}^{+0.016}$ & ${ }_{-0.012}^{+0.012}$ & ${ }_{-0.0086}^{+0.0086}$ & \\
\hline
\end{tabular}




\subsubsection{Alternative ratios $\alpha_{s}^{c} / \alpha_{s}^{u d s}$ and $\alpha_{s}^{b} / \alpha_{s}^{u d s}$}

An alternative, and perhaps more sensible, way to quote our result is in terms of the ratios $\alpha_{s}^{c} / \alpha_{s}^{u d s}$ and $\alpha_{s}^{b} / \alpha_{s}^{u d s}$. The procedure is very similar to that described in the previous sections; we solved the equations:

$$
\begin{aligned}
\mathcal{R}^{i} \equiv \frac{R_{3}^{i}}{R_{3}^{u d s}} & =\frac{A^{i} \bar{\alpha}_{s}^{i}+\left(B^{i}+C^{i}\right)\left(\bar{\alpha}_{s}^{i}\right)^{2}}{A^{0} \bar{\alpha}_{s}^{u d s}+\left(B^{0}+C^{0}\right)\left(\bar{\alpha}_{s}^{u d s}\right)^{2}} \\
& =\frac{A^{i} r_{i}^{\prime}+\bar{\alpha}_{s}^{u d s}\left(B^{i}+C^{i}\right) r_{i}^{\prime 2}}{A^{0}+\bar{\alpha}_{s}^{u d s}\left(B^{0}+C^{0}\right)}
\end{aligned}
$$

where $r_{i}^{\prime} \equiv \alpha_{s}^{i} / \alpha_{s}^{u d s}(i=c$ or $b)$, and we used $\alpha_{s}^{u d s} \equiv 0.118^{3}$. We performed a similar analysis to that described in previous sections using instead of $R_{3}^{u d s} / R_{3}^{\text {all }}, R_{3}^{c} / R_{3}^{\text {all }}$ and $R_{3}^{b} / R_{3}^{\text {all }}$, our measured vales of $R_{3}^{c} / R_{3}^{u d s}$ and $R_{3}^{b} / R_{3}^{u d s}$ as a starting point. These results of $R_{3}^{c} / R_{3}^{u d s}$ and $R_{3}^{b} / R_{3}^{u d s}$ are listed in Table 5.21, along with the various errors. The systematic errors were rederived; as an example, for the E0 algorithms and $y_{\text {cut }}=0.02$, the experimental systematic errors and hadronization uncertainties are listed in Table 5.19.

Eqn. 5.39 were solved analytically to obtain the ratios $r_{i}^{\prime}$. The example error matrices are:

$$
V(\mathcal{R})=\left(\begin{array}{ll}
0.0021354 & 0.0002121 \\
0.0002121 & 0.0003758
\end{array}\right), \quad V\left(r^{\prime}\right)=\left(\begin{array}{ll}
0.0013947 & 0.0001307 \\
0.0001307 & 0.0002183
\end{array}\right)
$$

The central values of $r_{i}^{\prime}$ and the statistical errors are listed in Table 5.21, and shown in Fig. 5.24. The experimental errors and hadronization uncertainties on on $r_{i}^{\prime}$ are listed in Table. 5.21. The translation uncertainties were reevaluated in this framework and are summarized in Table 5.20 .

Following the procedure of the previous section we obtained

$$
\begin{aligned}
& \alpha_{s}^{c} / \alpha_{s}^{u d s}=1.036 \pm 0.043(\text { stat. })_{-0.045}^{+0.041}(\text { syst. })_{-0.018}^{+0.020}(\text { theory }) \\
& \alpha_{s}^{b} / \alpha_{s}^{u d s}=1.004 \pm 0.018(\text { stat. })_{-0.031}^{+0.026}(\text { syst. })_{-0.029}^{+0.018}(\text { theory })
\end{aligned}
$$

\footnotetext{
${ }^{3} \mathrm{~A}$ large number of measurements below the threshold for $c$ and $b$ production contribute to the worldaverage value of $\alpha_{s}^{a l l}\left(M_{Z}^{2}\right)$, which is derived under the assumption of massless quarks. It is assumed that heavy-quark mass and possible flavor-dependent effects do not bias the world average value appreciably, so that we can take $\alpha_{s}^{u d s}\left(M_{Z}^{2}\right)=\alpha_{s}^{a l l}\left(M_{Z}^{2}\right)$; any such effects would be negligible on the scale of the errors considered on $\alpha_{s}\left(M_{Z}^{2}\right)$ (Table 5.16).
}

Table 5.19: Compilation of the systematic errors for the E0 algorithm and $y_{c u t}=0.02$. The

\begin{tabular}{|c|c|c|c|c|c|c|}
\hline \multicolumn{7}{|c|}{ E0 algorithm } \\
\hline source & $\begin{array}{l}\text { Center } \\
\text { Value }\end{array}$ & Variation & $\begin{array}{l}\delta R_{3}^{c} / \\
+\end{array}$ & $\overline{R_{3}^{u d s}}$ & $\begin{aligned} & \delta R_{3}^{b} / \\
&+\end{aligned}$ & $\overline{R_{3}^{u d s}}$ \\
\hline tracking efficiency & correction & off & 0.0020 & & -0.0110 & \\
\hline 2D imp. par. res. & smear & off & -0.0100 & & 0.0080 & \\
\hline $\mathrm{z}$ track resolution & smear & off & 0.0010 & & 0.0120 & \\
\hline MC statistics & $0.8 \mathrm{M}$ & & 0.0190 & -0.0190 & 0.0091 & -0.0091 \\
\hline$B$ decay $\left\langle n_{c h}\right\rangle$ & 5.51 & \pm 0.35 trks & -0.0030 & -0.0026 & 0.0135 & -0.0132 \\
\hline$B$ fragm. $\left\langle x_{b}\right\rangle$ & 0.697 & \pm 0.008 & $<0.0001$ & 0.0004 & 0.0172 & -0.0191 \\
\hline$B$ fragm. shape & Peterson & Bowler & 0.0021 & & -0.0216 & \\
\hline$B$ meson lifetime $\tau_{B}$ & $1.56 \mathrm{ps}$ & $\pm 0.05 p s$ & -0.0021 & 0.0022 & -0.0011 & 0.0009 \\
\hline$B$ baryon lifetime $\tau_{B}$ & $1.10 \mathrm{ps}$ & $\pm 0.08 \mathrm{ps}$ & -0.0003 & 0.0003 & $<0.0001$ & -0.0000 \\
\hline$B$ baryon prod. & $7.6 \%$ & $\pm 3.2 \%$ & 0.0014 & -0.0016 & 0.0021 & -0.0023 \\
\hline$B \rightarrow D^{+}+X$ frac. & 0.192 & \pm 0.05 & 0.0011 & -0.0012 & -0.0013 & -0.0008 \\
\hline$R_{b}$ & 0.2156 & \pm 0.0017 & 0.0022 & -0.0021 & 0.0014 & -0.0014 \\
\hline$R_{c}$ & 0.172 & \pm 0.010 & 0.0272 & -0.0294 & 0.0044 & -0.0042 \\
\hline$C$ fragm. $\left\langle x_{c}\right\rangle$ & 0.483 & \pm 0.008 & 0.0213 & -0.0211 & 0.0002 & -0.0002 \\
\hline$C$ fragm. shape & Peterson & Bowler & 0.0042 & & 0.0006 & \\
\hline$D^{0}$ decay $\left\langle n_{c h}>\right.$ & 2.54 & $\pm 0.06 \mathrm{trks}$ & 0.0044 & -0.0048 & 0.0006 & -0.0006 \\
\hline$D^{+}$decay $\left\langle n_{c h}\right\rangle$ & 2.48 & \pm 0.06 trks & 0.0069 & -0.0074 & 0.0012 & -0.0013 \\
\hline$D_{s}$ decay $\left\langle n_{c h}\right\rangle$ & 2.62 & \pm 0.31 trks & 0.0039 & -0.0040 & -0.0004 & 0.0003 \\
\hline$D^{0}$ lifetime & $0.418 p s$ & $\pm 0.004 \mathrm{ps}$ & -0.0001 & 0.0001 & -0.0002 & 0.0001 \\
\hline$D^{+}$lifetime & $1.054 \mathrm{ps}$ & $\pm 0.015 \mathrm{ps}$ & 0.0001 & -0.0001 & -0.0001 & 0.0001 \\
\hline$D_{s}$ lifetime & $0.466 \mathrm{ps}$ & $\pm 0.017 \mathrm{ps}$ & 0.0001 & -0.0001 & -0.0003 & 0.0003 \\
\hline$D^{0} \rightarrow K^{0}$ mult. & 0.402 & \pm 0.059 & 0.0088 & -0.0089 & 0.0026 & -0.0026 \\
\hline$D^{+} \rightarrow K^{0}$ mult. & 0.644 & \pm 0.078 & 0.0102 & -0.0120 & 0.0027 & -0.0027 \\
\hline$D_{s} \rightarrow K^{0}$ mult. & 0.382 & \pm 0.057 & 0.0012 & -0.0013 & 0.0003 & -0.0003 \\
\hline$D^{0} \rightarrow n o \pi^{0}$ & 0.370 & \pm 0.037 & 0.0069 & -0.0075 & 0.0034 & -0.0034 \\
\hline$D^{+} \rightarrow n o \pi^{0}$ & 0.496 & \pm 0.050 & 0.0017 & -0.0018 & 0.0029 & -0.0029 \\
\hline$D_{s} \rightarrow n o \pi^{0}$ & 0.348 & \pm 0.035 & -0.0002 & 0.0001 & -0.0003 & 0.0003 \\
\hline$c \bar{c} \rightarrow D^{+}+X$ frac. & 0.259 & \pm 0.028 & 0.0029 & -0.0034 & 0.0001 & -0.0002 \\
\hline$c \bar{c} \rightarrow D_{s}+X$ frac. & 0.113 & \pm 0.037 & -0.0025 & 0.0019 & 0.0002 & -0.0002 \\
\hline$c \bar{c} \rightarrow \Lambda_{c}+X$ & 0.074 & \pm 0.029 & -0.0051 & 0.0044 & -0.0001 & -0.0001 \\
\hline$\Lambda_{c}$ decay $<n_{c h}>$ & 2.79 & \pm 0.45 trks & 0.0003 & -0.0002 & 0.0024 & -0.0024 \\
\hline$\Lambda_{c}$ lifetime & $0.216 p s$ & $\pm 0.011 p s$ & -0.0037 & 0.0011 & -0.0006 & 0.0001 \\
\hline$g \rightarrow b b$ & 0.31 & $\pm 0.11 \%$ & 0.0001 & -0.0001 & -0.0038 & 0.0039 \\
\hline$g \rightarrow c c$ & 2.38 & $\pm 0.48 \%$ & -0.0019 & 0.0020 & -0.0015 & 0.0016 \\
\hline$K^{0}$ prodn. & 0.658 trks & \pm 0.050 trks & -0.0051 & 0.0045 & -0.0061 & 0.0058 \\
\hline$\Lambda$ prodn. & 0.124 trks & \pm 0.008 trks & -0.0007 & 0.0009 & -0.0008 & 0.0009 \\
\hline Total Exp. Syst. & & & 0.0440 & -0.0480 & 0.0300 & -0.0370 \\
\hline Q0 & $1 \mathrm{GeV}$ & $+1-0.5 \mathrm{GeV}$ & 0.0074 & -0.0027 & 0.0062 & -0.0237 \\
\hline & $0.39 \mathrm{GeV}$ & $\pm 0.04 \mathrm{GeV}$ & 0.0042 & -0.0008 & 0.0015 & 0.0012 \\
\hline had & JETSET7.4 & HERWIG5.9 & 0.0123 & & -0.0383 & \\
\hline Total Hadronization & & & 0.0150 & -0.0028 & 0.0065 & -0.0450 \\
\hline
\end{tabular}
first column shows the error source, the second column the central value used, and the third column the variation considered. The remaining columns show the corresponding errors on the values of $R_{3}^{c} / R_{3}^{u d s}$ and $R_{3}^{b} / R_{3}^{u d s}$; '+' ('-') denotes the error corresponding to the relevant positive (negative) parameter variation. 
Table 5.20: Summary of translation uncertainties on $\alpha_{s}^{i} / \alpha_{s}^{u d s}$ for each algorithm; '+' ('-') denotes the error corresponding to the relevant positive (negative) parameter variation.

\begin{tabular}{|c|c|c|c|c|c|c|}
\hline \multirow[t]{2}{*}{ source } & \multirow[t]{2}{*}{ Center } & \multirow[t]{2}{*}{ Variation } & \multicolumn{2}{|c|}{$\delta \alpha_{s}^{c} / \alpha_{s}^{a l l}$} & \multicolumn{2}{|c|}{$\delta \alpha_{s}^{b} / \alpha_{s}^{a l l}$} \\
\hline & & & + & - & + & - \\
\hline & \multicolumn{6}{|c|}{ E-algo $\left(y_{\text {cut }}=0.04\right)$} \\
\hline$m_{b}\left(M_{Z}\right)$ & $3.0 \mathrm{GeV}$ & \pm 0.5 & 0.000 & 0.000 & -0.014 & 0.015 \\
\hline$\mu, \alpha_{s}$ dep. & & & -0.002 & 0.001 & 0.006 & -0.006 \\
\hline$\geq 4$ jet contrib. & C & $\pm C$ & $<0.001$ & $<0.001$ & -0.001 & 0.001 \\
\hline \multirow[t]{2}{*}{ Total } & & & 0.001 & -0.002 & 0.016 & -0.015 \\
\hline & \multicolumn{6}{|c|}{ E0-algo $\left(y_{c u t}=0.02\right)$} \\
\hline$m_{b}\left(M_{Z}\right)$ & $3.0 \mathrm{GeV}$ & \pm 0.5 & 0.000 & 0.000 & -0.014 & 0.012 \\
\hline$\mu, \alpha_{s}$ dep. & & & -0.005 & 0.004 & 0.005 & -0.005 \\
\hline$\geq 4$ jet contrib. & C & $\pm C$ & -0.002 & 0.003 & -0.001 & 0.002 \\
\hline \multirow[t]{2}{*}{ Total } & & & 0.005 & -0.006 & 0.013 & -0.015 \\
\hline & \multicolumn{6}{|c|}{ P-algo $\left(y_{\text {cut }}=0.02\right)$} \\
\hline$m_{b}\left(M_{Z}\right)$ & $3.0 \mathrm{GeV}$ & \pm 0.5 & 0.000 & 0.000 & -0.012 & 0.009 \\
\hline$\mu, \alpha_{s}$ dep. & & & $<0.001$ & $<0.001$ & -0.002 & 0.002 \\
\hline$\geq 4$ jet contrib. & C & $\pm C$ & $<0.001$ & $<0.001$ & -0.005 & 0.007 \\
\hline \multirow[t]{2}{*}{ Total } & & & $<0.001$ & $<0.001$ & 0.011 & -0.014 \\
\hline & \multicolumn{6}{|c|}{$\mathrm{P} 0$-algo $\left(y_{\text {cut }}=0.015\right)$} \\
\hline$m_{b}\left(M_{Z}\right)$ & $3.0 \mathrm{GeV}$ & \pm 0.5 & 0.000 & 0.000 & -0.017 & 0.015 \\
\hline$\mu, \alpha_{s}$ dep. & & & -0.007 & 0.005 & -0.001 & $<0.000$ \\
\hline$\geq 4$ jet contrib. & C & $\pm C$ & -0.004 & 0.006 & -0.006 & 0.008 \\
\hline \multirow[t]{2}{*}{ Total } & & & 0.008 & -0.008 & 0.017 & -0.018 \\
\hline & \multicolumn{6}{|c|}{ D-algo $\left(y_{c u t}=0.010\right)$} \\
\hline$m_{b}\left(M_{Z}\right)$ & $3.0 \mathrm{GeV}$ & \pm 0.5 & 0.000 & 0.000 & 0.011 & -0.010 \\
\hline$\mu, \alpha_{s}$ dep. & & & -0.005 & 0.002 & -0.005 & 0.003 \\
\hline$\geq 4$ jet contrib. & C & $\pm C$ & -0.002 & 0.002 & -0.003 & 0.003 \\
\hline \multirow[t]{2}{*}{ Total } & & & 0.003 & -0.005 & 0.012 & -0.012 \\
\hline & \multicolumn{6}{|c|}{ G-algo $\left(y_{c u t}=0.080\right)$} \\
\hline$m_{b}\left(M_{Z}\right)$ & $3.0 \mathrm{GeV}$ & \pm 0.5 & 0.000 & 0.000 & 0.010 & -0.009 \\
\hline$\mu, \alpha_{s}$ dep. & & & -0.005 & 0.003 & 0.005 & -0.003 \\
\hline$\geq 4$ jet contrib. & $C$ & $\pm C$ & -0.002 & 0.003 & 0.001 & -0.001 \\
\hline Total & & & 0.004 & -0.006 & 0.008 & -0.009 \\
\hline
\end{tabular}

Table 5.21: $R_{3}^{i} / R_{3}^{u d s}$ and $\alpha_{s}^{i} / \alpha_{s}^{u d s}$ values and errors.

\begin{tabular}{|c|c|c|c|c|c|c|c|}
\hline $\begin{array}{l}\text { Algorithm } \\
y_{c}\end{array}$ & $\begin{array}{c}E \\
0.040\end{array}$ & $\begin{array}{c}\text { E0 } \\
0.020 \\
\end{array}$ & $\begin{array}{c}\mathrm{P} \\
0.020 \\
\end{array}$ & $\begin{array}{c}\text { P0 } \\
0.015 \\
\end{array}$ & $\begin{array}{c}\mathrm{D} \\
0.010 \\
\end{array}$ & $\begin{array}{c}\mathrm{G} \\
0.080 \\
\end{array}$ & \\
\hline \multicolumn{7}{|c|}{$R_{3}^{c} / R_{3}^{u d s}$} & \\
\hline central val. & 1.043 & 1.066 & 1.004 & 1.058 & 1.038 & 1.040 & \\
\hline stat. & 0.064 & 0.046 & 0.046 & 0.040 & 0.062 & 0.086 & \\
\hline exp. syst. & $\begin{array}{l}+0.065 \\
-0.075 \\
\end{array}$ & \begin{tabular}{l|}
+0.044 \\
-0.048 \\
\end{tabular} & $\begin{array}{l}+0.046 \\
-0.046 \\
\end{array}$ & $\begin{array}{l}+0.039 \\
-0.039 \\
\end{array}$ & $\begin{array}{l}+0.062 \\
-0.067 \\
\end{array}$ & $\begin{array}{l}+0.074 \\
-0.085 \\
\end{array}$ & \\
\hline hadronisation & $\begin{array}{l}+0.012 \\
-0.001 \\
\end{array}$ & \begin{tabular}{l|}
+0.015 \\
-0.003 \\
\end{tabular} & $\begin{array}{l}{ }_{-0.004}^{+0.014} \\
\end{array}$ & $\begin{array}{l}+0.015 \\
-0.003 \\
\end{array}$ & $\begin{array}{l}+0.006 \\
-0.003 \\
\end{array}$ & $\begin{array}{l}+0.008 \\
-0.004 \\
\end{array}$ & \\
\hline total. & $\begin{array}{l}{ }_{-0.099}^{+0.099} \\
\end{array}$ & \begin{tabular}{l|}
+0.065 \\
-0.067 \\
\end{tabular} & $\begin{array}{l}+0.067 \\
-0.065 \\
\end{array}$ & $\begin{array}{l}+0.058 \\
-0.056 \\
\end{array}$ & $\begin{array}{l}{ }_{-0.091}^{+0.088} \\
\end{array}$ & $\begin{array}{l}+0.114 \\
-0.121 \\
\end{array}$ & \\
\hline \multicolumn{7}{|c|}{$\alpha_{s}^{c} / \alpha_{s}^{u d s}$} & r.m.s \\
\hline central val. & 1.031 & 1.054 & 1.004 & 1.052 & 1.032 & 1.035 & 0.017 \\
\hline stat. & 0.046 & 0.037 & 0.041 & 0.035 & 0.051 & 0.074 & \\
\hline exp. syst. & $\begin{array}{l}0.047 \\
-0.054 \\
\end{array}$ & $\begin{array}{l}+0.036 \\
-0.039 \\
\end{array}$ & $\begin{array}{l}+0.041 \\
-0.041 \\
\end{array}$ & $\begin{array}{l}+0.035 \\
-0.035 \\
\end{array}$ & $\begin{array}{l}+0.051 \\
-0.055 \\
\end{array}$ & $\begin{array}{l}+0.064 \\
-0.073 \\
\end{array}$ & \\
\hline hadronisation & $\begin{array}{l}+0.009 \\
-0.001 \\
\end{array}$ & \begin{tabular}{l|}
+0.012 \\
-0.002 \\
\end{tabular} & $\begin{array}{l}+0.012 \\
-0.001 \\
\end{array}$ & $\begin{array}{l}+0.013 \\
-0.003 \\
\end{array}$ & $\begin{array}{l}{ }_{-0.002}^{+0.005} \\
\end{array}$ & $\begin{array}{l}+0.007 \\
-0.001 \\
\end{array}$ & \\
\hline translation & $\begin{array}{l}0.001 \\
-0.002 \\
\end{array}$ & \begin{tabular}{l|}
+0.005 \\
-0.006 \\
\end{tabular} & $<0.001$ & $\begin{array}{l}+0.008 \\
-0.008 \\
\end{array}$ & $\begin{array}{l}0.003 \\
-0.005 \\
\end{array}$ & $\begin{array}{l}+0.004 \\
-0.006 \\
\end{array}$ & \\
\hline \multicolumn{7}{|c|}{$R_{3}^{b} / R_{3}^{u d s}$} & \\
\hline central val. & 1.050 & 1.054 & 1.048 & 1.055 & 0.964 & 0.995 & \\
\hline stat. & 0.026 & 0.019 & 0.019 & 0.017 & 0.023 & 0.032 & \\
\hline exp. syst. & $\begin{array}{l}{ }_{-0.042}^{+0.038} \\
\end{array}$ & $\begin{array}{l}+0.030 \\
-0.037 \\
\end{array}$ & $\begin{array}{l}+0.027 \\
-0.037 \\
\end{array}$ & $\begin{array}{l}+0.028 \\
-0.035 \\
\end{array}$ & $\begin{array}{l}{ }_{-0.041}^{+0.038} \\
\end{array}$ & $\begin{array}{l}+0.035 \\
-0.036 \\
\end{array}$ & \\
\hline hadronisation & $\begin{array}{l}0.011 \\
-0.046 \\
\end{array}$ & $\begin{array}{l}+0.007 \\
-0.045 \\
\end{array}$ & $\begin{array}{l}+0.002 \\
-0.026 \\
\end{array}$ & $\begin{array}{l}+0.007 \\
-0.037 \\
\end{array}$ & $\begin{array}{l}0.001 \\
-0.006 \\
\end{array}$ & $\begin{array}{l}+0.020 \\
-0.008 \\
\end{array}$ & \\
\hline total. & $\begin{array}{l}0.047 \\
-0.067 \\
\end{array}$ & \begin{tabular}{l|}
+0.036 \\
-0.061 \\
\end{tabular} & $\begin{array}{l}+0.033 \\
-0.049 \\
\end{array}$ & $\begin{array}{l}+0.033 \\
-0.054 \\
\end{array}$ & $\begin{array}{l}+0.044 \\
-0.047 \\
\end{array}$ & $\begin{array}{l}+0.051 \\
-0.049 \\
\end{array}$ & \\
\hline \multicolumn{7}{|c|}{$\alpha_{s}^{b} / \alpha_{s}^{u d s}$} & r.m.s \\
\hline central val. & 0.989 & 0.995 & 1.018 & 1.014 & 1.009 & 0.993 & 0.011 \\
\hline stat. & 0.018 & 0.015 & 0.017 & 0.015 & 0.021 & 0.027 & \\
\hline exp. syst. & $\begin{array}{l}+0.026 \\
-0.029 \\
\end{array}$ & $\begin{array}{l}+0.023 \\
-0.028 \\
\end{array}$ & $\begin{array}{l}+0.023 \\
-0.032 \\
\end{array}$ & $\begin{array}{l}+0.024 \\
-0.030 \\
\end{array}$ & $\begin{array}{l}0.034 \\
-0.036 \\
\end{array}$ & $\begin{array}{l}+0.030 \\
-0.031 \\
\end{array}$ & \\
\hline hadronisation & $\begin{array}{l}{ }_{-0.032}^{+0.008} \\
\end{array}$ & $\begin{array}{l}+0.005 \\
-0.034 \\
\end{array}$ & $\begin{array}{l}+0.002 \\
-0.022 \\
\end{array}$ & $\begin{array}{l}+0.006 \\
-0.032 \\
\end{array}$ & $\begin{array}{l}{ }_{-0.005}^{+0.001} \\
\end{array}$ & $\begin{array}{l}+0.017 \\
-0.007 \\
\end{array}$ & \\
\hline translation & $\begin{array}{l}0.016 \\
{ }_{-0.015} \\
\end{array}$ & \begin{tabular}{l|}
+0.013 \\
-0.015 \\
\end{tabular} & $\begin{array}{l}+0.011 \\
-0.014 \\
\end{array}$ & $\begin{array}{l}+0.017 \\
-0.018 \\
\end{array}$ & $\begin{array}{l}0.012 \\
-0.012 \\
\end{array}$ & $\begin{array}{l}+0.008 \\
-0.009 \\
\end{array}$ & \\
\hline
\end{tabular}



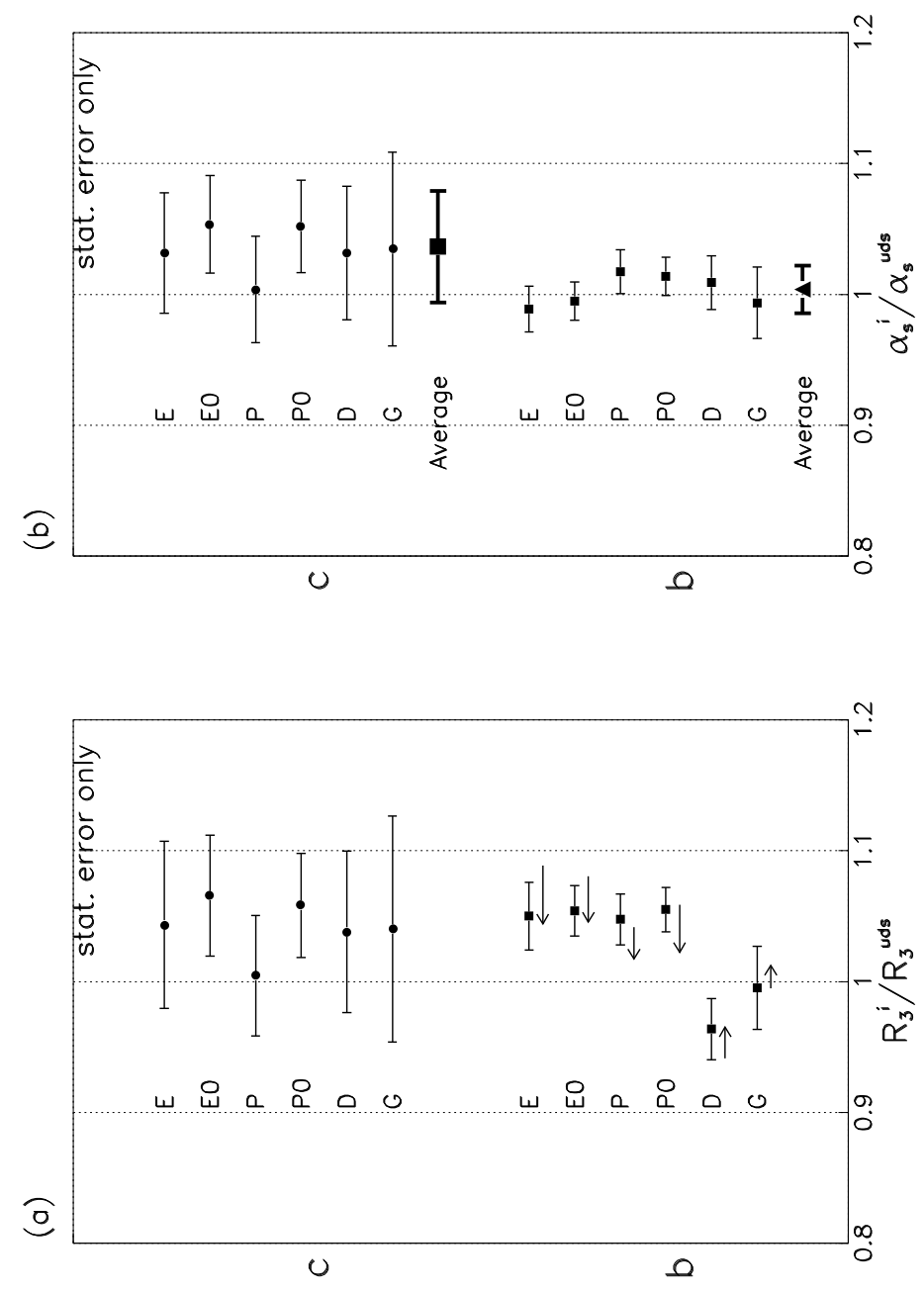

Figure 5.24: (a) The measured ratios $R_{3}^{i} / R_{3}^{u d s}$, and (b) the corresponding translated ratios $\alpha_{s}^{i} / \alpha_{s}^{u d s}(i=c, b)$. The arrows in (a) indicate the range of the theoretical prediction described in the text for values of the $b$-quark mass in the range $2.5 \leq m_{b}\left(M_{Z}\right) \leq 3.5 \mathrm{GeV} / c^{2}$, with the arrow pointing towards the lower mass value. In (b) the weighted average over the six algorithms is also shown. In all cases only statistical error bars are displayed.

\subsection{Cross Checks}

We performed a number of cross-checks on these results. First, we varied the event selection requirements. The thrust-axis containment cut was varied in the range $0.65<\left|\cos \theta_{T}\right|<$ 0.75 , the minimum number of charged tracks required was increased from 7 to 8 and the total charged-track energy requirement was increased from 20 to $22 \mathrm{GeV}$. In each case results consistent with the standard selection were obtained.

Next, we considered variations of the flavor-tagging scheme. In this section, we will describe the variation based on topological secondary vertex reconstruction as well as conventional lifetime flavor tagging.

\subsubsection{Variation of Topological Vertex Tagging}

We investigated several variations of flavor tagging based on topological SV reconstruction. Here, we briefly discuss these flavor tagging criteria. Those performances are compared in Table 5.24.

(0) We included in the unfolding procedure (Eq. 5.11 and Section 5.3.2) the 'untagged' event sample, subsample 4 (Section 4.2.3), whose flavor composition is similar to the natural composition in flavor-inclusive $Z^{0}$ decay events, and repeated the analysis to derive new values of $\alpha_{s}$ ratios. As a result, the correlations between flavors on $R_{3}$ are doubled.

(i) First, in the same unfolding procedure as the standard one, we used a more efficient tag for primary $b \bar{b}$ and $c \bar{c}$ events. We applied the scheme described in Section 4.2.3, but with a looser definition of region (A): $M_{v t x}>1.8$ OR $P_{v t x}+10<15 M_{v t x}$, then events containing any vertex in region (A) were re-defined to comprise the $b$ tagged sample (subsample 3). We also removed the cut on the vertex impact parameter $\delta_{v t x}$, and defined those events in region (B) to comprise the $c$-tagged sample (subsample 2). The $u d s$-tagged sample (subsample 1) consists of the events containing no SV with $N_{\text {sig }}=0$ is unchanged. The rest of the hadronic events (subsample 4) comprises only events with $N_{s i g} \geq 1$ and containing a SV with re-defined region $(\mathrm{C}) ; P_{v t x}>5$ and $P_{v t x}+10>15 M_{v t x}$. 
This method classified a $c$-contaminated $b$-rich region into the $b$-tag subsample. As a result, this increased the $b$-sample efficiency by $8 \%$ but decreased the purity by about $6 \%$. In addition, the $c-b$ correlation was doubled compared with the standard method

(ii) Second, we repeated this modified scheme (i), but changed the definition of tracks contribution to $N_{s i g}$ to include only those that miss the IP by $3 \sigma_{\delta}$, This loosens the lifetime anti-tag criterion for the $u d s$ tagged sample (subsample 1). Subsamples 2 and 3 are the same as (i)

Method (ii) loosens the lifetime anti-tag criteria for the $u d s$-tagged subsample. The looser significance from the IP contaminates the $u d s$ tagged subsample by the decay particles from long lifetime $D$ and $B$ hadrons. It turned out that $u d s$ efficiency increased by $25 \%$, but the $u d s-b$ and $c-b$ correlation were nearly doubled compared with method (i).

(iii) Third, we did not use vertex momentum information for the tag definitions; we used instead only vertex mass information to re-define region (A): $M_{v t x}>1.8$, and region (B): $M_{v t x}<1.8$. $b$-tagged sample (subsample 3 ) now re-defined to contain any SV in region (A), and rest of events containing SV are defined as $c$-tagged sample (subsample 2 ). The $u d s$-tagged sample (subsample 1) is the same as (ii), the rest is re-defined as subsample 4 . This criteria loosens the separation between $c$ - and $b$-tagged subsamples and the $c-b$ mixed samples are classified into the $c$-tagged subsample. As a result, the purity decreases $16 \%$ although the efficiency increases $7 \%$ in the $c$-tagged subsample.

Method (iii) does not use the momentum information of the SV, i.e. this method classifies the $b$-rich area below $M_{v t x}=1.8 \mathrm{GeV}$ in region $(\mathrm{C})$ into $c$-tagged subsample This significantly worsened the purity of the $c$-tagged subsample.

(iv) Finally, we tried a variation of (iii), in which we used event hemispheres as a basis for flavor-tagging, rather than jets as defined in Sect. 4.2; this tag is similar to that used in our recent study of the branching fraction for $Z^{0} \rightarrow b \bar{b}[110]$.

The efficiencies and purities are very similar to method (iii) but the flavor-tagging biases for $c$ and $b$ are significantly larger than those of jet-based tagging methods.

Fig. 5.29 shows the results of various cross checks.

\subsubsection{Lifetime-based Tag}

We also performed an analysis using a similar flavor-tagging technique to that reported in [9], namely made use of the properties of long-lived $B$ and $C$ hadrons without SV reconstruction. We counted the number of flavor-tagging tracks per event, $N_{s i g}$, that miss the IP by $d / \sigma_{d} \geq 3$. This distiribution is shown in Fig. 5.25; the data are well described by our Monte Carlo simulation. For the simulation, the contributions of events of different quark flavors are shown separately. The leftmost bin contains predominantly events containing primary $u, d$, or $s$ quarks, while the rightmost bins contain a pure sample of events containing primary $b$-quarks. The event sample was divided accordingly into five subsamples according to the number of 'significant' tracks: (1) $N_{s i g}=0$, (2) $N_{s i g}=1$, (3) $N_{s i g}=2$, (4) $N_{s i g}=3$, and (5) $N_{s i g} \geq 4$. We refer to subsample 1 as the uds-tagged sample, the union of subsamples 2,3 and 4 as the c-tagged sample, and subsample 5 as the b-tagged sample.

The hard $b$ tag yields a sample with very low contamination from charm events, maximizing the sensitivity of the three-flavor test. The efficiencies $\varepsilon$ for selecting events (after cuts) of type $i(i=u d s, c, b)$ with $\operatorname{tag} j$, and the fractions $\Pi$ of events of type $i$ in the $j$-tagged sample, were calculated from the Monte Carlo simulation to be: $(\varepsilon, \Pi)_{u d s}=(84.2 \pm 0.1 \%, 86.2 \pm 0.1 \%)$, $(\varepsilon, \Pi)_{c}=(57.0 \pm 0.2 \%, 32.3 \pm 0.1 \%),(\varepsilon, \Pi)_{b}=(43.4 \pm 0.2 \%, 95.2 \pm 0.1 \%)$. These are compared with those of the standard one in Table 5.24.

As an example for the E0 algorithm Fig. 5.26 shows $R_{3}^{j} / R_{3}^{\text {all }}$ for each subsample $j(1 \leq$ $j \leq 5)$ as a function of $y_{c u t}$; where $R_{3}^{\text {all }}$ is the $\geq 3$-jet rate in the total event sample, and the unfolded ratios $R_{3}^{i} / R_{3}^{\text {all }}(i=u d s, c, b)$ using a similar formalism to that defined by Eqn.5.11. For each algorithm the unfolded ratios $R_{3}^{i} / R_{3}^{\text {all }}$ are shown in Fig. 5.27. At the chosen $y_{\text {cut }}$, the $R_{3}$ ratios listed in Table 5.22 were then translated to $\alpha_{s}$ ratios using Eqn. 5.25. Fig. 5.28 illustrates those ratios for the six algorithms; some scattering on $c$-ratios presumably comes from statistical fluctuation due to relatively lower purity $(\sim 30 \%)$ for $c$-tagged subsamples.

We considered major experimental systematic effects that could modify the tagging efficiencies. The errors are summarized in Table 5.23, where averages over the six algorithms are shown. The dominant physics contributions in $\alpha_{s}^{b} / \alpha_{s}^{\text {all }}$ result from limited knowledge of the average $B$ decay multiplicity and the heavy quark fragmentation functions. The uncertainty in $B R\left(Z^{0} \rightarrow c \bar{c}\right)$ also produces variations in $\alpha_{s}^{c} / \alpha_{s}^{\text {all }}$ and $\alpha_{s}^{u d s} / \alpha_{s}^{\text {all }}$. The detector systematic error is dominated by the uncertainty in the charged track reconstruction efficiency. 
Following the procedure described in Sect. 5.5.4 we obtained :

$$
\begin{aligned}
\frac{\alpha_{s}^{u d s}}{\alpha_{s}^{a l l}} & =0.997 \pm 0.011 \text { (stat) } \pm 0.011 \text { (syst) } \pm 0.008 \text { (theory) } \\
\frac{\alpha_{s}^{c}}{\alpha_{s}^{\text {all }}} & =0.984 \pm 0.042 \text { (stat) } \pm 0.053 \text { (syst) } \pm 0.044 \text { (theory) } \\
\frac{\alpha_{s}^{b}}{\alpha_{s}^{\text {all }}} & =1.022 \pm 0.019 \text { (stat) } \pm 0.023 \text { (syst) } \pm 0.025 \text { (theory) }
\end{aligned}
$$

The theoretical uncertainties include r.m.s. over 6 algorithms. This result is also consistent with the other results (Fig. 5.29), but has larger statistical and systematic errors (Table 5.24).

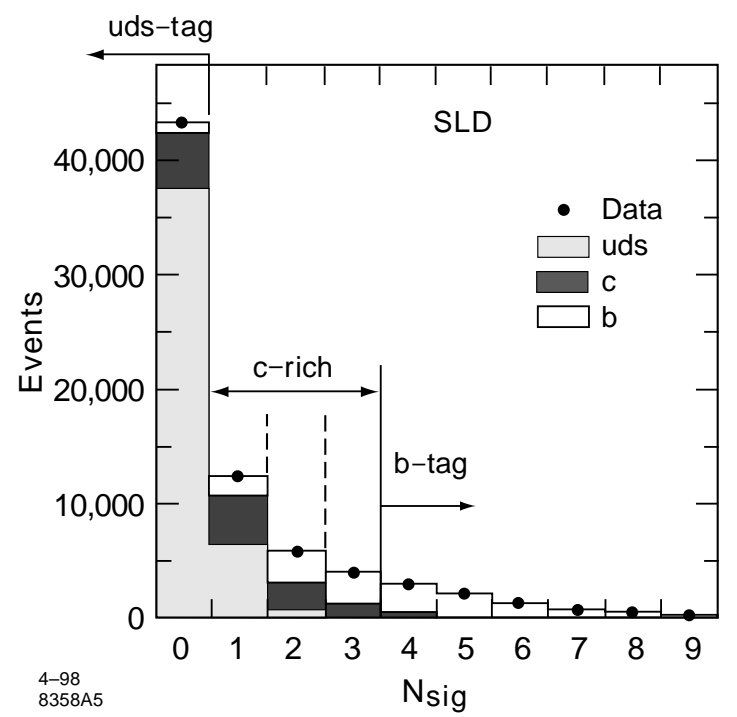

Figure 5.25: The distribution of the the number of tracks that miss the IP by at least $3 \sigma$ in terms of their impact parameter in the plane normal to the beamline: data (points); the simulated distribution is shown as a histogram in which the contributions from events of different primary quark flavor are indicated.

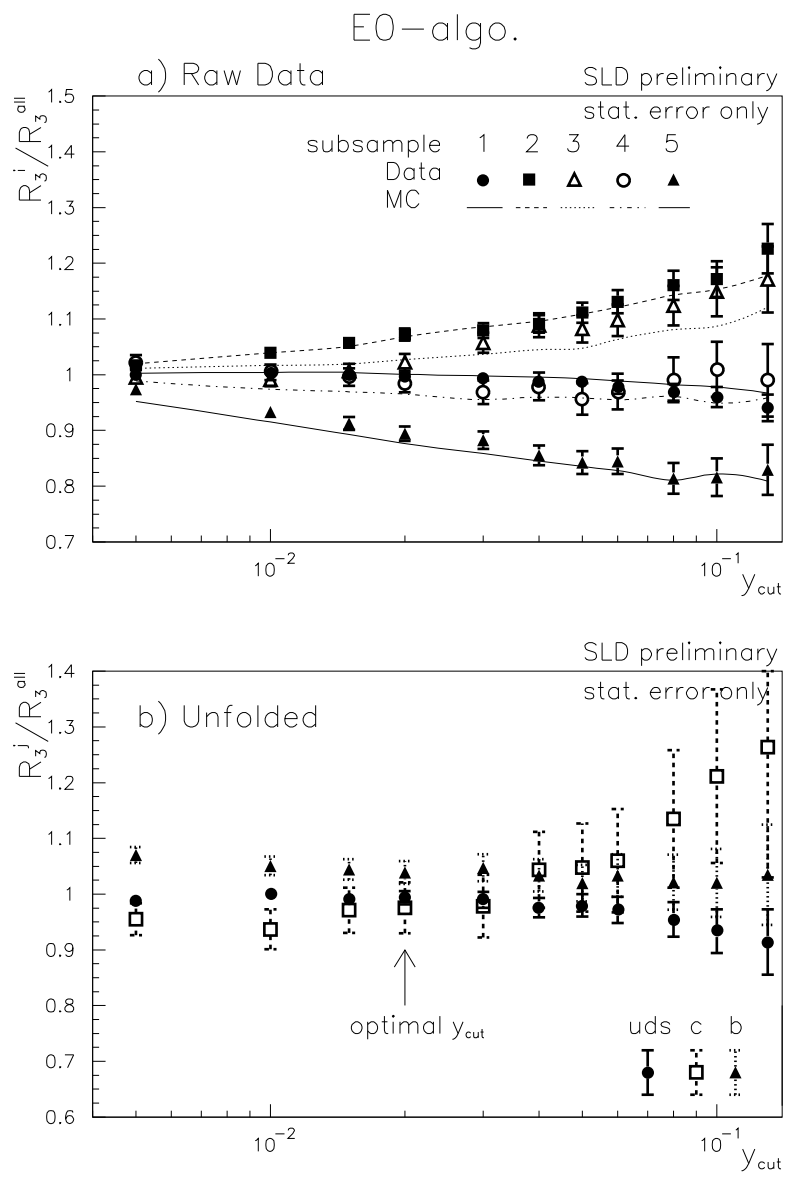

Figure 5.26: (a) The raw measured ratios $R_{3}^{j} / R_{3}^{\text {all }}, 1 \leq j \leq 5$, vs. $y_{\text {cut }}$ for the 5 subsamples classified by $N_{s i g}$ (see text); data (points with error bars), and simulation (lines joining values at the same $y_{\text {cut }}$ values as the data). (b) The unfolded $R_{3}^{i} / R_{3}^{a l l}, i=u d s, c, b$, vs. $y_{\text {cut }}$ for the 3 primary event flavor groups. Only statistical errors are shown. The arrow indicate the chosen $y_{\text {cut }}$ value. 

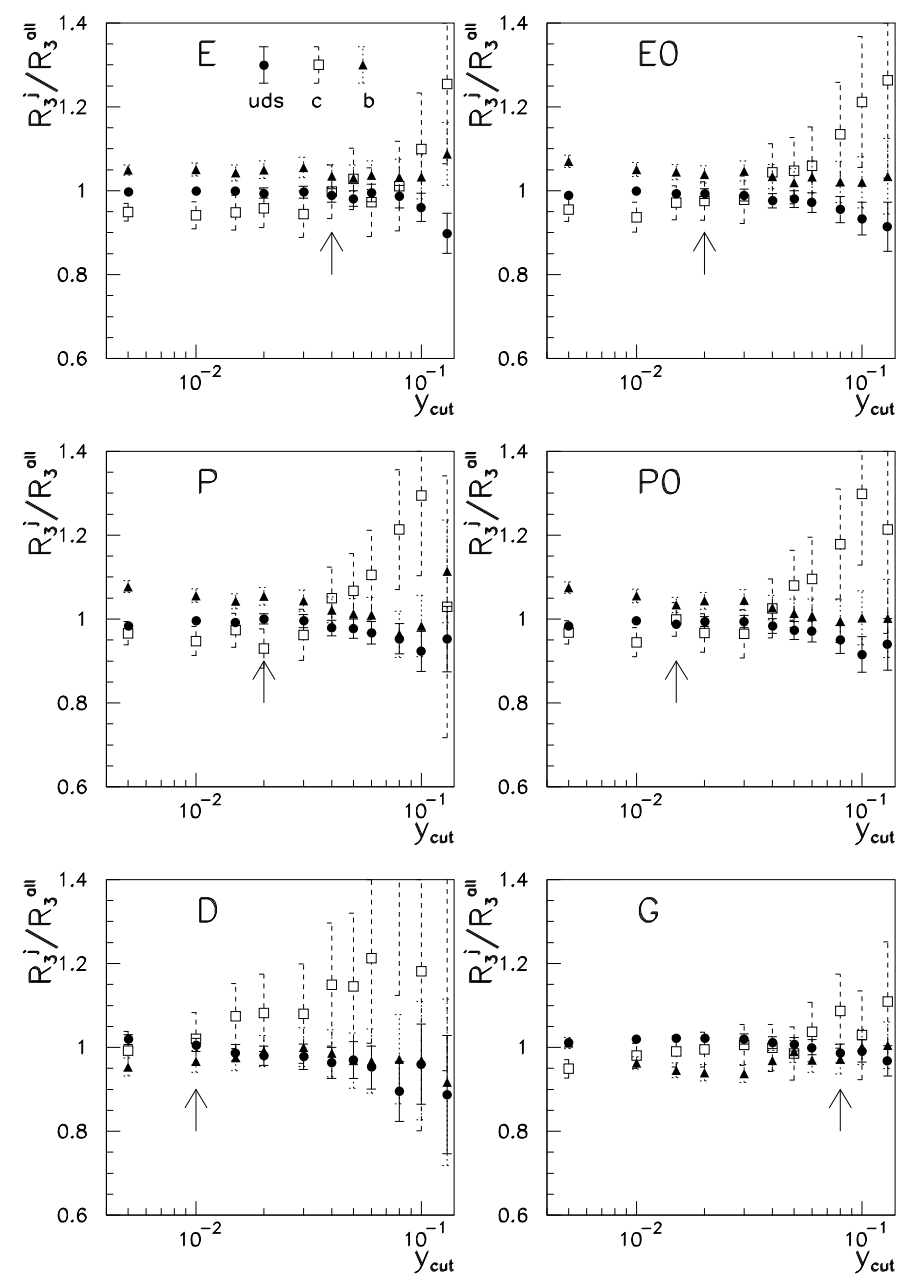

Figure 5.27: The unfolded ratios $R_{3}^{i} / R_{3}^{a l l}, i=u d s, c, b$, vs. $y_{\text {cut }}$ for each algorithms using impact parameter tag. Only statistical errors are shown. Arrows indicate the chosen $y_{\text {cut }}$ values.
Table 5.22: Results of $R_{3}^{i} / R_{3}^{\text {all }}(i=u d s, c, b)$ using lifetime (impact parameter) tag. The errors shown are statistical only.

\begin{tabular}{ccccc}
\hline Algorithm & $y_{\text {cut }}$ & $R_{3}^{\text {uds }} / R_{3}^{\text {all }}$ & $R_{3}^{c} / R_{3}^{\text {all }}$ & $R_{3}^{b} / R_{3}^{\text {all }}$ \\
\hline E & 0.040 & $0.989 \pm 0.016$ & $0.998 \pm 0.064$ & $1.035 \pm 0.027$ \\
E0 & 0.020 & $0.993 \pm 0.012$ & $0.975 \pm 0.046$ & $1.039 \pm 0.020$ \\
P & 0.020 & $1.001 \pm 0.012$ & $0.930 \pm 0.047$ & $1.054 \pm 0.021$ \\
P0 & 0.015 & $0.989 \pm 0.010$ & $0.999 \pm 0.040$ & $1.034 \pm 0.018$ \\
D & 0.010 & $1.006 \pm 0.016$ & $1.021 \pm 0.062$ & $0.966 \pm 0.026$ \\
G & 0.080 & $0.986 \pm 0.022$ & $1.086 \pm 0.088$ & $0.972 \pm 0.035$ \\
\hline
\end{tabular}

Table 5.23: Systematic errors on the results of $\alpha_{s}^{i} / \alpha_{s}^{\text {all }}$ using lifetime tag. The first column shows the error source, the second column the central value used, and the third column the variation considered. The remaining columns show the corresponding errors on the values of $\alpha_{s}^{i} / \alpha_{s}^{\text {all }}(i=u d s, c, b)$.

\begin{tabular}{|l||c|c|c|c|c|}
\hline source & Center Value & Variation & $\Delta\left(\frac{\alpha_{s}^{u d s}}{\alpha_{s}^{a d l}}\right)$ & $\Delta\left(\frac{\alpha_{a l}^{c}}{\alpha_{s}^{a l}}\right)$ & $\Delta\left(\frac{\alpha_{s}^{b}}{\alpha_{s}^{b}}\right)$ \\
\hline tracking efficiency & correction & on/off & 0.002 & 0.013 & 0.005 \\
impact parameter resolution & smear & on/off & 0.001 & 0.005 & 0.001 \\
\hline MC statistics & $0.8 \mathrm{M}$ events & & 0.005 & 0.020 & 0.008 \\
\hline$B$ decay multiplicity $<n_{c h}>$ & 5.39 & \pm 0.2 trks & 0.001 & 0.022 & 0.014 \\
$B$ fragmentation $<x_{b}>$ & 0.700 & \pm 0.008 & 0.002 & 0.008 & 0.012 \\
$B$ meson lifetime $\tau_{B}$ & $1.55 p s$ & $\pm 0.05 p s$ & 0.001 & 0.008 & 0.003 \\
$B$ baryon lifetime $\tau_{B}$ & $1.10 p s$ & $\pm 0.08 p s$ & 0.001 & 0.002 & 0.001 \\
$B$ baryon prod. rate $f_{\Lambda b}$ & $7 \%$ & $\pm 4 \%$ & 0.001 & 0.004 & 0.006 \\
$B \rightarrow D^{+}+X$ fraction & 0.15 & \pm 0.05 & 0.001 & 0.003 & 0.002 \\
$Z^{0} \rightarrow b \bar{b}: f^{b}$ & 0.2216 & \pm 0.0017 & 0.001 & 0.003 & 0.001 \\
\hline$Z^{0} \rightarrow c \bar{c}: f^{c}$ & 0.17 & \pm 0.010 & 0.0008 & 0.037 & 0.005 \\
$C$ fragmentation $<x_{c}>$ & 0.484 & \pm 0.008 & 0.003 & 0.011 & 0.002 \\
$c \bar{c} \rightarrow D^{+}+X$ fraction & 0.20 & \pm 0.04 & 0.001 & 0.003 & 0.001 \\
$C$ decay multiplicity $<n_{c h}>$ & 2.34 & \pm 0.2 trks & 0.003 & 0.008 & 0.006 \\
\hline$g \rightarrow c c$ & $1.7 \times 10^{-2}$ & $\pm 50 \%$ & 0.001 & 0.002 & 0.002 \\
$g \rightarrow b b$ & $1.6 \times 10^{-3}$ & $\pm 50 \%$ & 0.001 & 0.001 & 0.003 \\
\hline Total & & & 0.011 & 0.053 & 0.023 \\
\hline
\end{tabular}




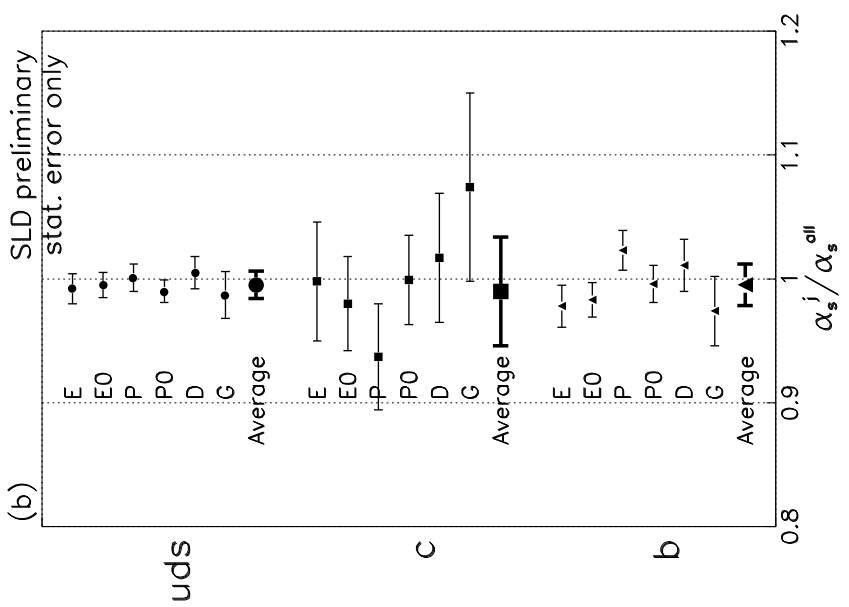

\subsubsection{Discussion}

Fig. 5.29 shows the results of the various cross checks. Note that all the results derived from different schemes are consistent within statistical errors.

Averaged over all six algorithms, those flavor tagging performances and statistical errors on the $\alpha_{s}$ ratios as well as correlation coefficients between flavors are listed in Table 5.24. None of these tags for cross checks gives smaller errors on the $\alpha_{s}$ ratios than the nominal scheme. The errors on the $\alpha_{s}$ ratios using variants of vertex mass tagging are about $20 \%$ better than those from lifetime tagging due to the relatively purified flavor tagged subsamples in the vertex mass tagging.

Fluctuations in $\alpha_{s}^{c} / \alpha_{s}^{\text {all }}$ are due to small statistics; this ratio is the most sensitive to the variation of the tagging criteria.

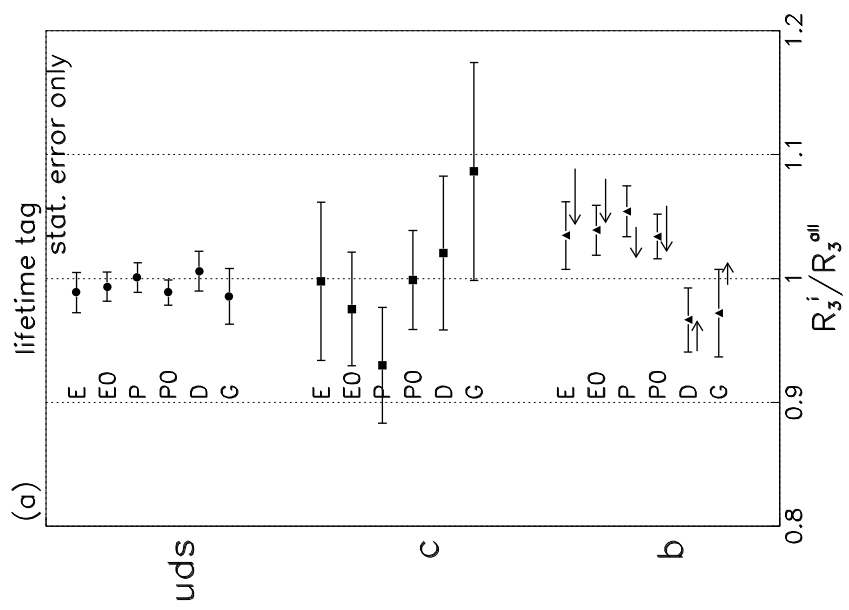

Table 5.24: (a) Comparison of tagging methods

\begin{tabular}{|c||c|c|c|c|c|c|c|c|}
\hline flavor & & VtxMass & $(0)$ & (i) & (ii) & (iii) & (iv) & lifetime tag \\
\hline \multirow{7}{*}{ uds } & eff. (\%) & 56.4 & 56.4 & 56.4 & 81.2 & 81.2 & 81.2 & 83.9 \\
& puri. (\%) & 90.6 & 90.6 & 90.6 & 87.4 & 87.4 & 87.4 & 85.8 \\
& bias. (\%) & 4.1 & 4.1 & 4.3 & -0.7 & -0.5 & -3.4 & -5.1 \\
& stat.err. (\%) & 1.0 & 0.9 & 1.0 & 1.0 & 1.0 & 1.0 & 1.1 \\
\hline \multirow{7}{*}{ charm } & eff. & 19.1 & 19.1 & 21.9 & 21.9 & 29.0 & 26.1 & 56.5 \\
& puri. & 64.4 & 64.4 & 62.1 & 62.1 & 46.4 & 49.7 & 31.9 \\
& bias & 14.5 & 14.5 & 16.2 & 16.2 & 4.4 & 23.4 & 9.8 \\
& stat.err. & 3.4 & 3.3 & 3.4 & 3.4 & 3.6 & 3.6 & 4.2 \\
\hline \multirow{6}{*}{ bottom } & eff. & 61.5 & 61.5 & 69.9 & 69.9 & 63.5 & 64.6 & 42.4 \\
& puri. & 95.5 & 95.5 & 89.8 & 89.8 & 93.3 & 93.7 & 95.1 \\
& bias. & 5.7 & 5.7 & 6.5 & 6.5 & 7.8 & 12.2 & 19.6 \\
& stat.err. & 1.6 & 1.5 & 1.6 & 1.6 & 1.6 & 1.6 & 1.9 \\
\hline
\end{tabular}

(b) tag correlation on $R$

Figure 5.28: (a) The measured ratios of $R_{3}^{i} / R_{3}^{\text {all }}$, and (b) translated ratios $\alpha_{s}^{i} / \alpha_{s}^{\text {all }}(i=$ $u d s, c, b)$ using lifetime (impact parameter) tag as a cross check (see text). The arrows in (a) indicate the range of the theoretical prediction described in the text for values of the $b$-quark mass in the range $2.5 \leq m_{b}\left(M_{Z}\right) \leq 3.5 \mathrm{GeV} / c^{2}$, with the arrow pointing towards the lower mass value. In (b) the weighted average over the six algorithms is also shown. In all cases only statistical error bars are displayed.

\begin{tabular}{|c||c|c|c|c|c|c|c|c|}
\hline \multicolumn{1}{|c|}{ (b) tag correlation on $R_{3}$} \\
\hline uds-c & $-39.6(\%)$ & -61.8 & -38.8 & -52.8 & -56.4 & 61.0 & -68 \\
uds-b & $4.0(\%)$ & 8.7 & 9.7 & 13.7 & 17.7 & 17.0 & 32 \\
c-b & $-18.6(\%)$ & -26.7 & -35.2 & -35.2 & -41.4 & -42.0 & -57 \\
\hline
\end{tabular}




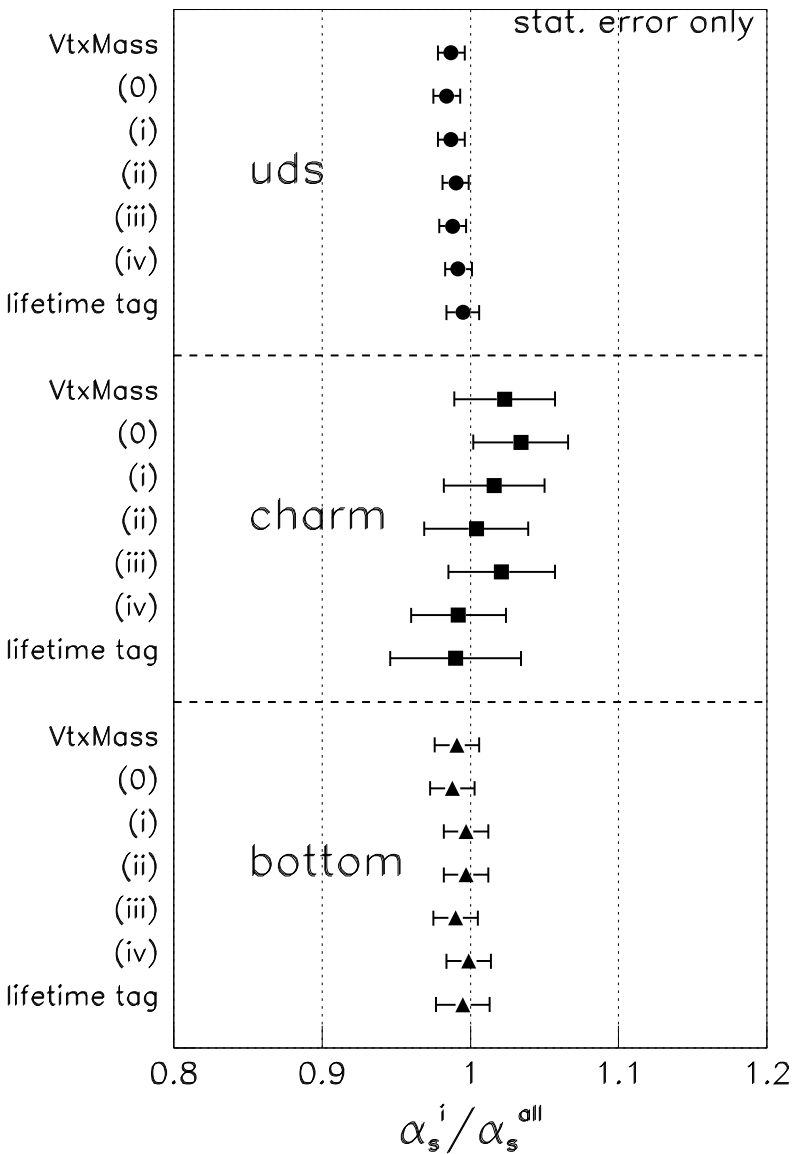

Figure 5.29: Summary of cross checks. Results for the standard flavor tagging, vertex mass reconstruction, and those variations (0-iv), as well as lifetime tag are shown (see text)

\section{Chapter 6}

\section{Summary, Discussion and Conclusions}

We measured the relative strength of the strong interaction between flavors using 150,000 hadronic $Z^{0}$ decays recorded with the SLC Large Detector at the SLAC Linear Collider between 1993 and 1995. Taking advantage of the CCD pixel based vertex detector and the tiny and stable beam interaction region, we have used the topological secondary vertex mass reconstruction technique along with hadron lifetime information as a basis of the flavor separation into three flavor samples $(u d s, c$, and $b)$, with high purities and efficiencies, and small biases against events containing 3-jets. The measured ratios of 3-jet rates using 6 different algorithms ( E, E0, P, P0, Durham, Geneva ) in different event flavor samples were translated to the ratios of strong couplings using recent next-to-leading order calculations of 3 -jet final states, including the effects of a non-zero $b$-quark mass. These effects are not small on the scale of our current errors and must be taken into account.

We obtained :

$$
\begin{aligned}
\alpha_{s}^{\text {uds }} / \alpha_{s}^{\text {all }} & =0.987 \pm 0.010(\text { stat. })_{-0.010}^{+0.012}(\text { syst } .)_{-0.008}^{+0.009}(\text { theory }) \\
\alpha_{s}^{c} / \alpha_{s}^{\text {all }} & =1.023 \pm 0.034(\text { stat. })_{-0.036}^{+0.032}(\text { syst. })_{-0.014}^{+0.018}(\text { theory }) \\
\alpha_{s}^{b} / \alpha_{s}^{\text {all }} & =0.993 \pm 0.016(\text { stat. })_{-0.023}^{+0.020}(\text { syst } .)_{-0.027}^{+0.019}(\text { theory })
\end{aligned}
$$

or alternatively

$$
\begin{aligned}
& \alpha_{s}^{c} / \alpha_{s}^{u d s}=1.036 \pm 0.043(\text { stat } .)_{-0.045}^{+0.041}(\text { syst. })_{-0.018}^{+0.020}(\text { theory }) \\
& \alpha_{s}^{b} / \alpha_{s}^{u d s}=1.004 \pm 0.018(\text { stat } .)_{-0.031}^{+0.026}(\text { syst } .)_{-0.029}^{+0.018}(\text { theory })
\end{aligned}
$$


These results are consistent with our previous measurements [9], as well as the updated results using the same technique, but are substantially more precise, as well as with measurements performed at LEP using different flavor-tagging techniques [6].

In order to compare our limits on flavor independence with those obtained by other experiments, we show our results on $\alpha_{s}^{i} / \alpha_{s}^{\text {all }}$ along with those from the LEP experiments in Figure 6.1; where necessary the published results of $\alpha_{s}^{b} / \alpha_{s}^{u d s c}$ for the other experiments [7, $67,69,72]$ are translated to our convention of $\alpha_{s}^{b} / \alpha_{s}^{\text {all }}$ taking into account $Z^{0} \rightarrow q \bar{q}$ branching fraction ${ }^{1}$.

As described in Sect. 2.4.1 a measurement of the relative strength between light flavors $(u, d$, and $s)$ is extremely difficult to achieve because it is inevitable to suffer from small statistics and large bias against 3-jet events. Whereas the most common approach is to assume that $\alpha_{s}^{u}=\alpha_{s}^{d}=\alpha_{s}^{s}=\alpha_{s}^{c}$ or $\alpha_{s}^{c}=\alpha_{s}^{b}$, we only make the weak assumption that $\alpha_{s}^{u}=\alpha_{s}^{d}=\alpha_{s}^{s}$. Note that the measurement of ratios has largely diminished the effects of the renormalization scale uncertainty and other errors common to an absolute $\alpha_{s}$ value measurement.

Our comprehensive study, involving six jet-finding algorithms, and inclusion of the resulting r.m.s. deviations of results as additional uncertainties, represents a conservative procedure. These ratios are consistent with unity, and hence the strong coupling is independent of quark flavor within our sensitivity.

${ }^{1}$ The OPAL results [7] are translated from the results on $\alpha_{s}^{i} / \alpha_{s}^{a l l}(i=u, d, s, c, b)$ and those correlations listed in Table 9 and Table 11 of [7], respectively, taking into account $Z^{0} \rightarrow q \bar{q}$ branching fraction.

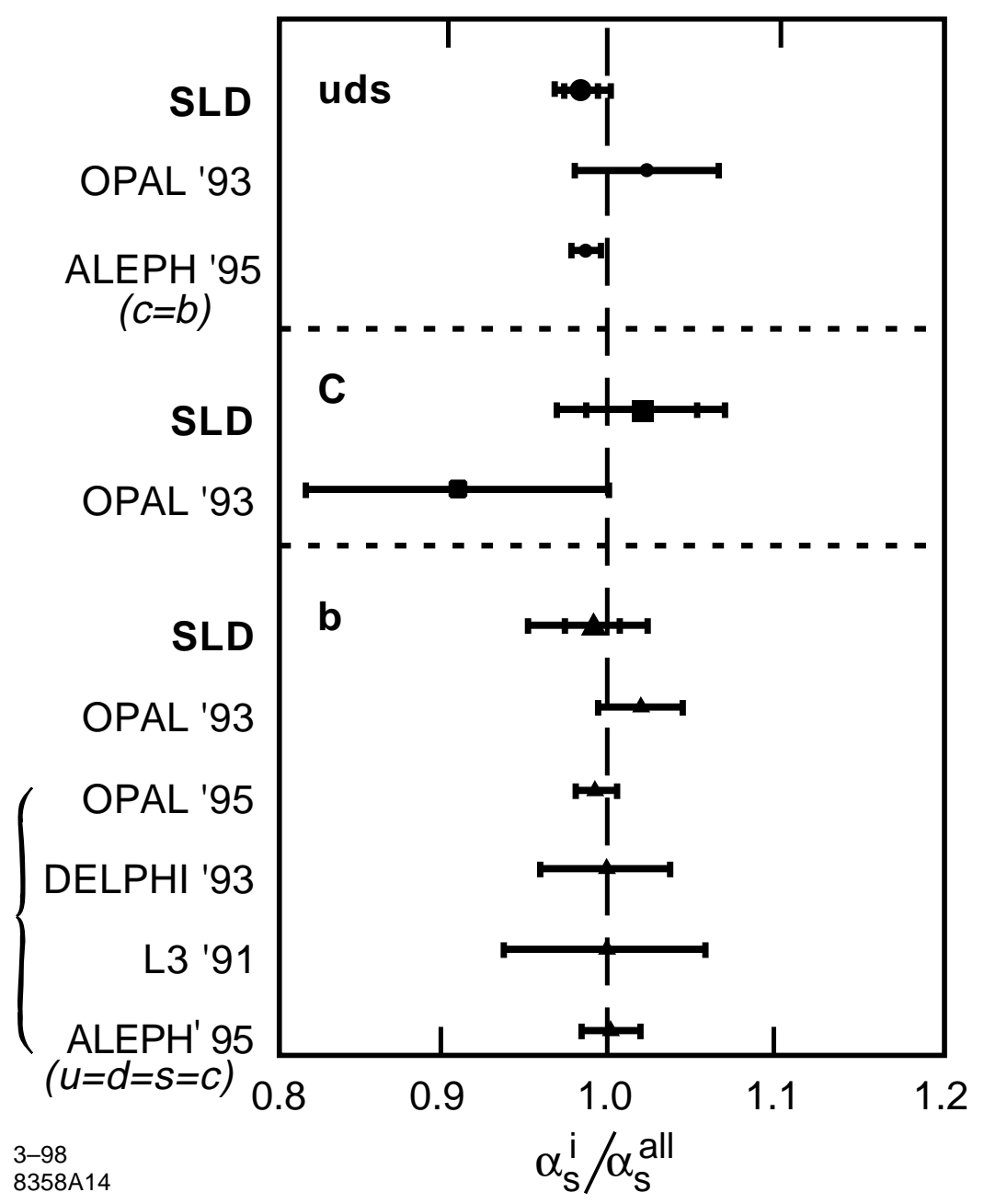

Figure 6.1: Summary of measurements of $\alpha_{s}^{i} / \alpha_{s}^{\text {all }}(i=u d s, c, b)$ from experiments at the $Z^{0}$ resonance. We derived the ALEPH $\alpha_{s}^{u d s} / \alpha_{s}^{a l l}$ value from their measured value of $\alpha_{s}^{u d s} / \alpha_{s}^{b c}$, as well as the file bracketed LEP values of $\alpha_{s}^{b} / \alpha_{s}^{a l l}$ from the measured values of $\alpha_{s}^{b} / \alpha_{s}^{u d s c}$, by assuming $\alpha_{s}^{\text {all }}=\sum_{u d s, c, b} f^{i} \alpha_{s}^{i}$, where $f^{i}$ is the Standard Model branching fraction for $Z^{0}$ decays to quark flavor $i$. 


\section{Appendix A}

\section{$V^{0}$ Rejection}

Tracks resulting both from decays of long lived neutral particles (so-called $V^{0}$ decay) such as $\Lambda$ 's and $K_{s}^{0}$ 's, and from interactions with the detector material ( $\gamma$-conversions), often have large impact parameters. They account for much of contaminations from light quark events in the $b$ - or $c$ - tagged events. Thus, it is necessary to identify $V^{0}$ and $\gamma$-conversions efficiently so that they may be eliminated from the tracks considered in the flavor-tagging while minimizing misidentification. The $\gamma$ conversion have a side benefit of being useful for verifying the simulation of the detector material. The rejection schemes for $V^{0}$ and $\gamma$-conversion described here is identical to the one used in [97].

\section{Identifying $K_{s}^{0}$ and $\Lambda$ decay}

The procedure for identifying $K_{s}^{0}$ and $\Lambda$ 's, uses all pairs of CDC tracks that pass the good track selection cuts and have opposite signed charges. A rough approximation of the $V^{0}$ decay position was assumed by taking the intersection points between the tracks treated as circles in the $x y$ plane. Only CDC track track information were used to this crude approximation, however, if the approximate position appeard to be below a radius of $2 \mathrm{~cm}$, then the combined CDC+VXD track information was used, and the position was recomputed. Next, the approximate vertex position was used as a seed for performing a complete 3-D vertex fit. Then the fitted parameters were used for the following cuts

- The angle between the vertex position vector taken from the IP to the $V^{0}$ decay vertex position, and the momentum vector sum of the $V^{0}$ tracks must be less than $0.2 \mathrm{rad}$ for
$K_{s}^{0}$ and $0.5 \mathrm{rad}$ for $\Lambda$, respectively

- The opening angle of the $V^{0}$ must be less than $1.3 \mathrm{rad}$

- The separation of the tracks in $x y$ at the reconstructed $V^{0}$ decay vertex position must be less than $0.02 \mathrm{~cm}$

- A cut on the longitudinal separation $(\delta)$ between the tracks at the reconstructed $V^{0}$ vertex position is applied with a dependence on the displacement from the IP $\left(R_{V^{0}}\right)$ : $\delta<0.3 \times R_{v^{0}}$.

- The invariant mass is determined and the $V^{0}$ passes if the mass is within $20 \mathrm{MeV}$ and $9 \mathrm{MeV}$ for the $K_{s}^{0}$ and $\Lambda$, respectively.

\section{Identifying Gamma Conversions}

The procedure for identifying $\gamma$ conversions was similar to that for $V^{0}$ except that the position of the conversion was determined by where the circle approximations of the corresponding tracks were parallel to each other in the $x y$ plane. The uncertainty in position along the $\gamma$ flight direction can be very large (several $\mathrm{cm}$ ) because of the very small opening angle resulting from massless parent particle(the $\gamma)$. A full vertex fit was not performed. Similarly to the procedure for $K_{s}^{0}$ and $\Lambda \mathrm{s}$, the following cuts were performed.

- The angle between the vector taken from the IP to the $\gamma$-conversion point, and the momentum vector sum of the converted tracks must be less than $0.2 \mathrm{rad}$.

- The opening angle of the converted tracks must be less than $0.2 \mathrm{rad}$

- The separation of the tracks in $x y$ at the vertex position must be less than $0.3 \mathrm{~cm}$

- The invariant mass is determined and the $\gamma$ is identified if this mas is less than $20 \mathrm{MeV}$

The reconstructed invariant masses are shown in Fig. A.1. Approximately 1, 0.5 and 1 tracks per event were rejected as $K_{s}^{0}, \Lambda$, and $\gamma$-conversion candidates, respectively. The number of $\gamma$-conversion is shown in Fig A.2 as a function of distance with respect to the beam-line. The simulation of the detector material well reproduces the data. 

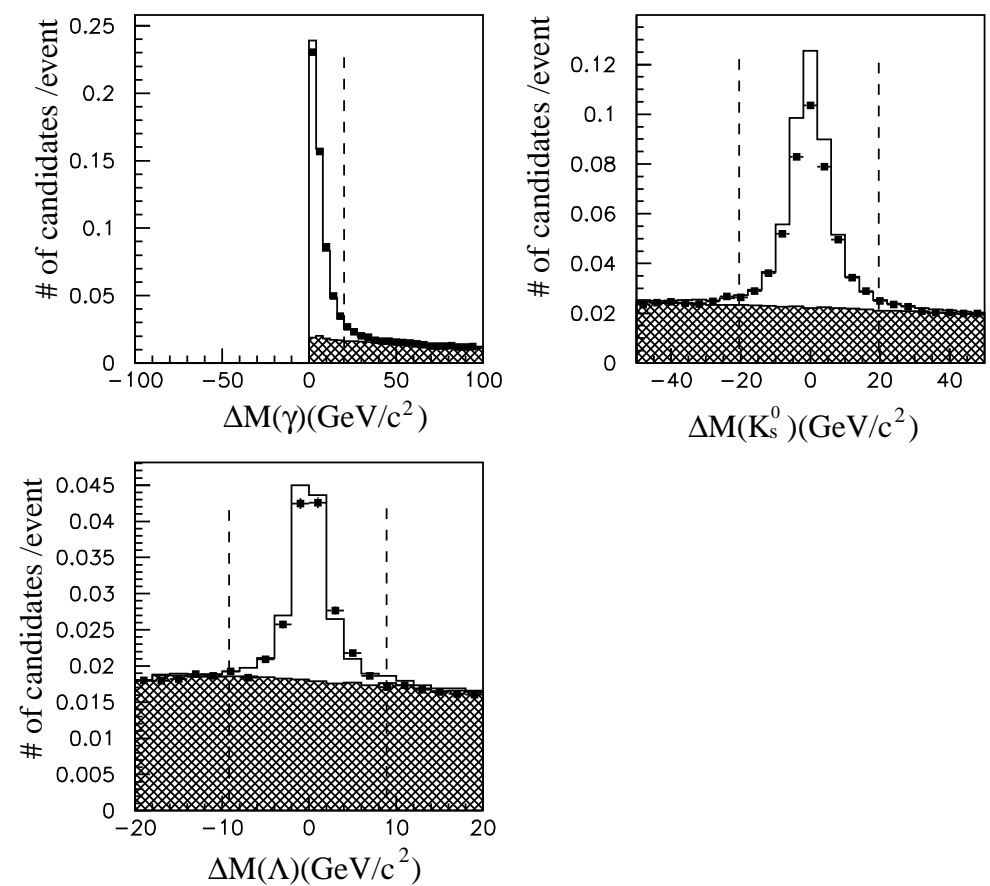

Figure A.1: Distribution of the difference between reconstructed mass and expect value of the identified $\gamma$-conversion and $V^{0}$ candidates. The points represent the data and the histogram represent the Monte Carlo, whose hatched area is the fraction of random combinatorial background.

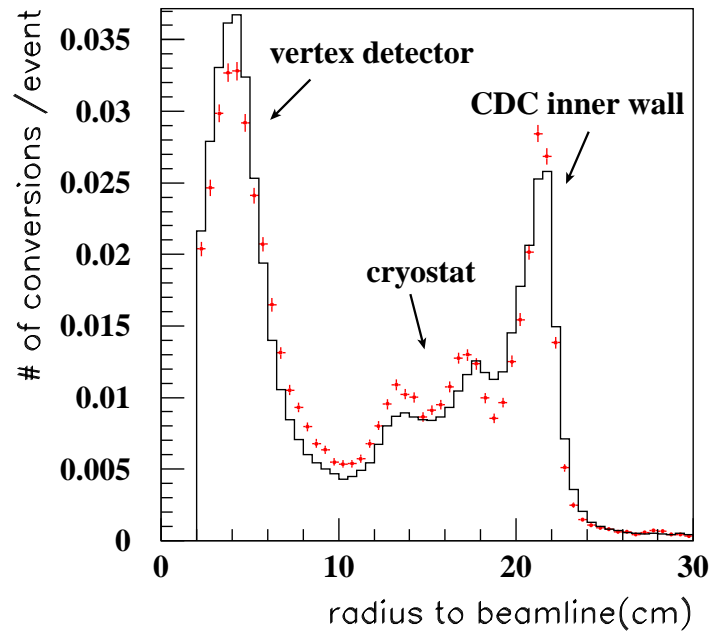

Figure A.2: Reconstructed gamma conversions as a function of distance from beam-line; the data (plots) and Monte Carlo(histogram). Peaks in the distribution correspond to the positions of the detector materials. 


\section{Appendix B}

\section{Unfolding Scheme using the Least}

\section{Squares Method}

The unfolding formula Eqn. 5.11:

$$
\begin{aligned}
& n_{2}^{j}=\sum_{i=1}^{3}\left(\varepsilon_{(2 \rightarrow 2)}^{j i}\left(1-R_{3}^{i}\right)+\varepsilon_{(3 \rightarrow 2)}^{j i} R_{3}^{i}\right) f_{i} N \\
& n_{3}^{j}=\sum_{i=1}^{3}\left(\varepsilon_{(3 \rightarrow 3)}^{j i} R_{3}^{i}+\varepsilon_{(2 \rightarrow 3)}^{j i}\left(1-R_{3}^{i}\right)\right) f_{i} N
\end{aligned}
$$

where the index $i$ refers to the primordial quark flavor; $j$ denotes the flavor tagged subsample. can be written as:

$$
\vec{Y}=\vec{A}+B \cdot \vec{r}
$$

where the elements of $\vec{Y}=\left(n_{2}^{1}, n_{2}^{2}, n_{2}^{3}, n_{3}^{1}, n_{3}^{2}, n_{3}^{3}\right)$ and is the number of 2- and 3-jet events in each flavor tagged subsamples, and the elements of $\vec{r}=\left(R_{3}^{1}, R_{3}^{2}, R_{3}^{3}\right)$ is the parton level 3 -jet rate $R_{3}^{i}$ for true flavors ( $u d s, c$, and $b$ ) to be solved.

\section{APPENDIX B. UNFOLDING SCHEME USING THE LEAST SQUARES METHOD 158}

The vector $\vec{A}$ and the matrix $B$ is given by:

$$
\vec{A}=N\left(\begin{array}{c}
\sum_{i}^{3} f_{i} \epsilon_{(2 \rightarrow 2)}^{1 i} \\
\sum_{i}^{3} f_{i} \epsilon_{(2 \rightarrow 2)}^{2 i} \\
\sum_{i}^{3} f_{i} \epsilon_{(2 \rightarrow 2)}^{3 i} \\
\sum_{i}^{3} f_{i} \epsilon_{(2 \rightarrow 3)}^{1 i} \\
\sum_{i}^{3} f_{i} \epsilon_{(2 \rightarrow 3)}^{2 i} \\
\sum_{i}^{3} f_{i} \epsilon_{(2 \rightarrow 3)}^{3 i}
\end{array}\right)
$$

$$
B=N\left(\begin{array}{lll}
\left(\epsilon_{(3 \rightarrow 2)}^{11}-\epsilon_{(2 \rightarrow 2)}^{11}\right) f_{1} & \left(\epsilon_{(3 \rightarrow 2)}^{12}-\epsilon_{(2 \rightarrow 2)}^{12}\right) f_{2} & \left(\epsilon_{(3 \rightarrow 2)}^{13}-\epsilon_{(2 \rightarrow 2)}^{13}\right) f_{3} \\
\left(\epsilon_{(3 \rightarrow 2)}^{21}-\epsilon_{(2 \rightarrow 2)}^{21}\right) f_{1} & \left(\epsilon_{(3 \rightarrow 2)}^{22}-\epsilon_{(2 \rightarrow 2)}^{22}\right) f_{2} & \left(\epsilon_{(3 \rightarrow 2)}^{23}-\epsilon_{(2 \rightarrow 2)}^{23}\right) f_{3} \\
\left(\epsilon_{(3 \rightarrow 2)}^{31}-\epsilon_{(2 \rightarrow 2)}^{31}\right) f_{1} & \left(\epsilon_{(3 \rightarrow 2)}^{32}-\epsilon_{(2 \rightarrow 2)}^{32}\right) f_{2} & \left(\epsilon_{(3 \rightarrow 2)}^{33}-\epsilon_{(2 \rightarrow 2)}^{33}\right) f_{3} \\
\left(\epsilon_{(3 \rightarrow 3)}^{11}-\epsilon_{(2 \rightarrow 3)}^{11}\right) f_{1} & \left(\epsilon_{(3 \rightarrow 3)}^{12}-\epsilon_{(2 \rightarrow 3)}^{12}\right) f_{2} & \left(\epsilon_{(3 \rightarrow 3)}^{13}-\epsilon_{(2 \rightarrow 3)}^{13}\right) f_{3} \\
\left(\epsilon_{(3 \rightarrow 3)}^{21}-\epsilon_{(2 \rightarrow 3)}^{21}\right) f_{1} & \left(\epsilon_{(3 \rightarrow 3)}^{22}-\epsilon_{(2 \rightarrow 3)}^{22}\right) f_{2} & \left(\epsilon_{(3 \rightarrow 3)}^{23}-\epsilon_{(2 \rightarrow 3)}^{23}\right) f_{3} \\
\left(\epsilon_{(3 \rightarrow 3)}^{31}-\epsilon_{(2 \rightarrow 3)}^{31}\right) f_{1} & \left(\epsilon_{(3 \rightarrow 3)}^{32}-\epsilon_{(2 \rightarrow 3)}^{32}\right) f_{2} & \left(\epsilon_{(3 \rightarrow 3)}^{33}-\epsilon_{(2 \rightarrow 3)}^{33}\right) f_{3}
\end{array}\right)
$$

In order to extract $\vec{r}$ which we want to know, we introduce the $\chi^{2}$ defined by:

$$
\chi^{2}=(\vec{Y}-\vec{A}-B \cdot \vec{r})^{T} W(\vec{Y}-\vec{A}-B \cdot \vec{r})
$$

where

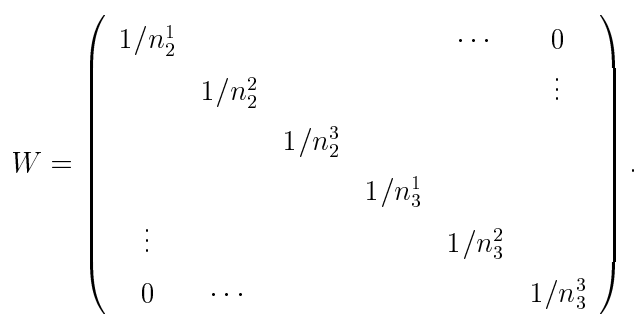

Minimizing $\chi^{2}$ require

$$
\frac{\partial \chi^{2}}{\partial \vec{r}}=-2 B^{T} W(\vec{Y}-\vec{A}-B \cdot \vec{r})=0
$$

Therefore, $\vec{r}$ can be obtained by

$$
\vec{r}=\left(B^{T} W B\right)^{-1} B^{T} W(\vec{Y}-\vec{A}) .
$$

The error matrix on $R_{3}^{i}$ is given by:

$$
V(\vec{r})=\left(B^{T} W B\right)^{-1}
$$


Approximately 50,000 hadronic decays of $Z^{0}$ were recorded in the SLD 1996 run and $\sim 300,000$ are expected during the current run in 1997-1998. The SLD collaboration is devoting a thorough effort to VXD3 alignment [74], however, our analysis of the flavor independence of strong interactions for the 1997-1998 data is too preliminary to include in this thesis. We expect that the uncertainty on $\alpha_{s}^{\text {flavors }} / \alpha_{s}^{\text {all }}$ could be reduced by a factor of 2 using the whole data sample.

\section{Appendix C}

\section{Possible Improvements with the}

\section{VXD3 detector}

Accumulated experience of the SLD vertex detector (VXD2) and semiconductor technology allowed us to design a new vertex detector (VXD3) [95]. In the beginning of 1996 VXD3 was installed in the SLD.

The $x y$ and $r z$ views of VXD3 are shown in Figures C.1 and C.2, respectively. A comparison between VXD3 and VXD2 is shown in Table C.1. VXD3 provides larger acceptance in both $x y$ and in $r z$ planes, less multiple scattering and higher efficiency. The impact parameter resolution is better than that of VXD2 by a factor of 2, which is shown in Fig. C.3 [95].

These features bring more accurate reconstruction of the secondary vertices and better flavor tagging. Table C.2 summarizes the flavor-tagging efficiencies and purities for this analysis using VXD3 compared with results from VXD2. The criteria used here are identical to the analysis for VXD2 data. Although there might be room to optimize it, $c$ tag efficiency and purity are drastically improved by about $10 \%$ and $4 \%$, respectively, and the $b$ tag efficiency is increased about $20 \%$ with similar purity. The analysis for the 1996 data is very preliminary :

$$
\begin{aligned}
\alpha_{s}^{\text {uds }} / \alpha_{s}^{\text {all }} & =0.982 \pm 0.015(\text { stat. }) \\
\alpha_{s}^{c} / \alpha_{s}^{a l l} & =1.043 \pm 0.047 \text { (stat. }) \\
\alpha_{s}^{b} / \alpha_{s}^{a l l} & =1.017 \pm 0.019 \text { (stat. })
\end{aligned}
$$

Table C.1: VXD3/VXD2 Comparison.

\begin{tabular}{|lcc|}
\hline Parameter & VXD2 & VXD3 \\
\hline \hline Motherboard material & Alumina & Beryllium \\
Number of CCDs & 480 & 96 \\
Pixels $/$ CCD & $400 \times 600$ & $800 \times 4000$ \\
Pixel size & $22 \mu \mathrm{m} \times 22 \mu \mathrm{m}$ & $20 \mu \mathrm{m} \times 20 \mu \mathrm{m}$ \\
Readout time & $160 \mathrm{~ms}$ & $210 \mathrm{~ms}$ \\
& $(19$ beam crossings $)$ & $(25$ beam crossings $)$ \\
Radius Layer 1 & $2.96 \mathrm{~cm}$ & $2.80 \mathrm{~cm}$ \\
Radius Layer 2 & $3.36 \mathrm{~cm}$ & $3.82 \mathrm{~cm}$ \\
Radius Layer 3 & $3.76 \mathrm{~cm}$ & $4.83 \mathrm{~cm}$ \\
Radius Layer 4 & $4.16 \mathrm{~cm}$ & - \\
Radiation thickness & $1.15 \% /$ layer & $0.36 \% /$ layer \\
Average hits per track & 2.3 & 3.0 \\
Two-hit coverage & $|\cos \theta|<0.75$ & $|\cos \theta|<0.90$ \\
\hline
\end{tabular}

note that these errors are statistical only. 


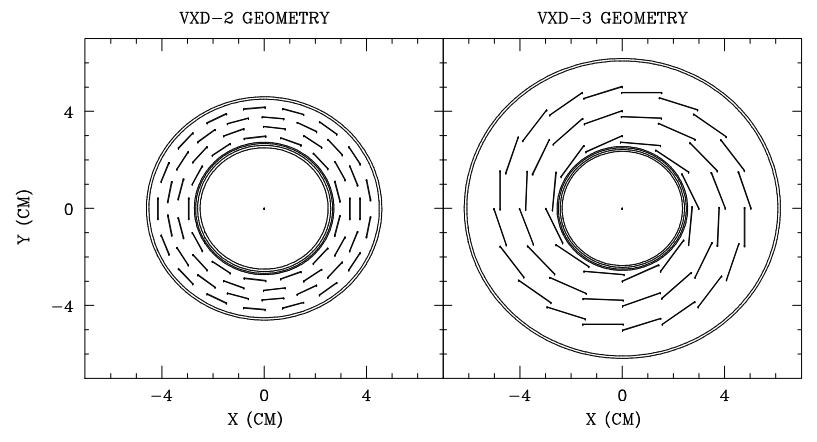

Figure C.1: Cross-section view ( $x y$ plane) of the VXD2 and VXD3.

\section{VXD3}
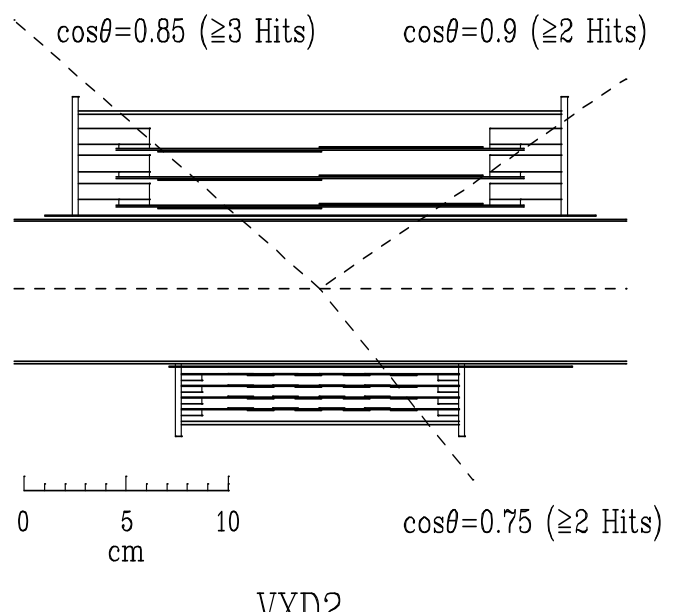

VXD2

Figure C.2: Cross-section view ( $r z$ plane) of the VXD2 and VXD3.
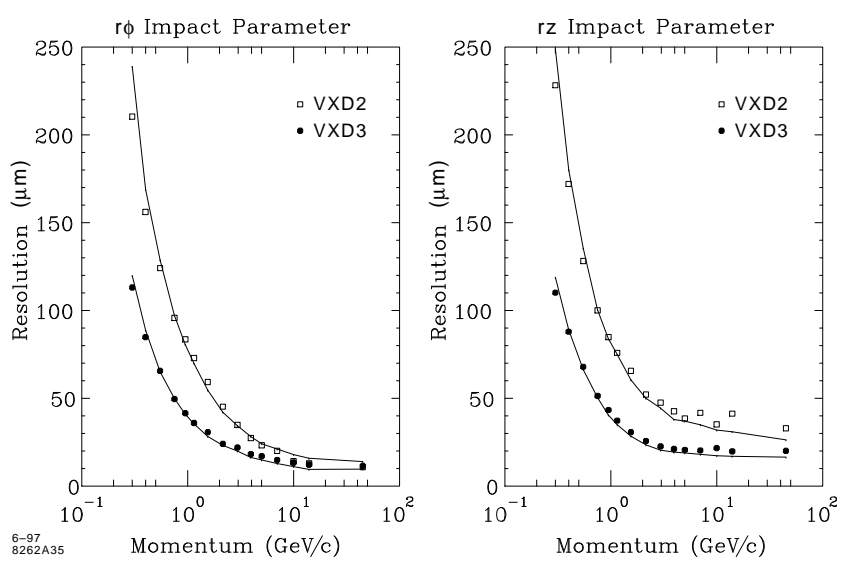

Figure C.3: Comparison of impact parameter resolution as a function of track momentum for tracks at $\cos \theta=0$ for VXD2 and VXD3, where $\theta$ is the polar angle w.r.t the beam-axis. Points are measured data and lines are the Monte Carlo simulations.

Table C.2: VXD3/VXD2 comparison for flavor tagging performance

\begin{tabular}{|c||c|c|c|}
\hline flavor & & VXD3 (1996 data) & VXD2 (1993-1995) \\
\hline \multirow{2}{*}{ uds } & eff. & $49.9(\%)$ & $56.4(\%)$ \\
& puri. & 92.4 & 90.6 \\
& bias. & 1.8 & 3.7 \\
& stat.err & 1.5 & 1.0 \\
\hline \multirow{2}{*}{ charm } & eff. & 23.6 & 19.1 \\
& puri. & 75.0 & 64.4 \\
& bias & 16.2 & 15.8 \\
& stat.err & 4.7 & 3.4 \\
\hline \multirow{2}{*}{ bottom } & eff. & 79.2 & 61.5 \\
& puri. & 91.0 & 95.5 \\
& bias. & 3.0 & 6.5 \\
& stat.err & 1.9 & 1.6 \\
\hline
\end{tabular}




\section{Appendix D}

\section{The SLD collabolation}

K. Abe, ${ }^{(29)}$ K. Abe, ${ }^{(17)}$ T. Abe, ${ }^{(25)}$ T. Akagi, ${ }^{(25)}$ N. J. Allen, ${ }^{(4)}$ A. Arodzero, ${ }^{(18)}$ D. Aston, ${ }^{(25)}$ K.G. Baird, ${ }^{(14)}$ C. Baltay, ${ }^{(35)}$ H.R. Band, ${ }^{(34)}$ T.L. Barklow, ${ }^{(25)}$ J.M. Bauer, ${ }^{(15)}$ A.O. Bazarko, ${ }^{(8)}$ G. Bellodi, ${ }^{(19)}$ A.C. Benvenuti, ${ }^{(3)}$ G.M. Bilei, ${ }^{(21)}$ D. Bisello, ${ }^{(20)}$ G. Blaylock, ${ }^{(14)}$ J.R. Bogart, ${ }^{(25)}$ T. Bolton ${ }^{(8)}$ G.R. Bower ${ }^{(25)}$ J. E. Brau, ${ }^{(18)}$ M. Breidenbach, ${ }^{(25)}$ W.M. Bugg, ${ }^{(28)}$ D. Burke, ${ }^{(25)}$ T.H. Burnett, ${ }^{(33)}$ P.N. Burrows, ${ }^{(19)}$ A. Calcaterra, ${ }^{(11)}$ D.O. Caldwell, ${ }^{(30)}$ D. Calloway, ${ }^{(25)}$ B. Camanzi, ${ }^{(10)}$ M. Carpinelli, ${ }^{(22)}$

R. Cassell, ${ }^{(25)}$ R. Castaldi, ${ }^{(22)}$ A. Castro, ${ }^{(20)}$ M. Cavalli-Sforza, ${ }^{(31)}$ A. Chou, ${ }^{(25)}$ H.O. Cohn, ${ }^{(28)}$ J.A. Coller, ${ }^{(5)}$ M.R. Convery, ${ }^{(25)}$ V. Cook, ${ }^{(33)}$ R.F. Cowan, ${ }^{(16)}$ D.G. Coyne, ${ }^{(31)}$ G. Crawford, ${ }^{(25)}$ C.J.S. Damerell, ${ }^{(23)}$ M. Daoudi, ${ }^{(25)}$ N. de Groot, ${ }^{(25)}$ R. Dell'Orso ${ }^{(21)}$ P.J. Dervan ${ }^{(4)}$ R. de Sangro ${ }^{(11)}$ M. Dima ${ }^{(9)}$ A. D'Oliveira ${ }^{(6)}$ D.N. Dong,${ }^{(16)}$ R. Dubois, ${ }^{(25)}$ B.I. Eisenstein, ${ }^{(12)}$ V. Eschenburg, ${ }^{(15)}$ E. Etzion, ${ }^{(34)}$ S. Fahey, ${ }^{(7)}$ D. Falciai, ${ }^{(11)}$ J.P. Fernandez, ${ }^{(31)}$ M.J. Fero, ${ }^{(16)}$ R. Frey ${ }^{(18)}$ G. Gladding, ${ }^{(12)}$

E.L. Hart, ${ }^{(28)}$ J.L. Harton, ${ }^{(9)}$ A. Hasan, ${ }^{(4)}$ K. Hasuko, ${ }^{(29)}$ S. J. Hedges, ${ }^{(5)}$ S.S. Hertzbach, ${ }^{(14}$

M.D. Hildreth, ${ }^{(25)}$ M.E. Huffer, ${ }^{(25)}$ E.W. Hughes, ${ }^{(25)}$ X.Huynh, ${ }^{(25)}$ M. Iwasaki, ${ }^{(18)}$

D. J. Jackson, ${ }^{(23)}$ P. Jacques, ${ }^{(24)}$ J.A. Jaros, ${ }^{(25)}$ Z.Y. Jiang, ${ }^{(25)}$ A.S. Johnson, ${ }^{(25)}$

J.R. Johnson, ${ }^{(34)}$ R.A. Johnson, ${ }^{(6)}$ R. Kajikawa, ${ }^{(17)}$ M. Kalelkar, ${ }^{(24)}$ Y. Kamyshkov, ${ }^{(28)}$ H.J. Kang, ${ }^{(24)}$ I. Karliner, ${ }^{(12)}$ Y. D. Kim, ${ }^{(26)}$ M.E. King, ${ }^{(25)}$ R.R. Kofler, ${ }^{(14)}$

R.S. Kroeger, ${ }^{(15)}$ M. Langston, ${ }^{(18)}$ D.W.G. Leith, ${ }^{(25)}$ V. Lia, ${ }^{(16)}$ X. Liu, ${ }^{(31)}$ M.X. Liu, ${ }^{(35)}$ M. Loreti, ${ }^{(20)}$ H.L. Lynch, ${ }^{(25)}$ G. Mancinelli, ${ }^{(24)}$ S. Manly, ${ }^{(35)}$ G. Mantovani, ${ }^{(21)}$ T.W. Markiewicz, ${ }^{(25)}$ T. Maruyama, ${ }^{(25)}$ H. Masuda, ${ }^{(25)}$ A.K. McKemey, ${ }^{(4)}$

B.T. Meadows, ${ }^{(6)}$ G. Menegatti, ${ }^{(10)}$ R. Messner, ${ }^{(25)}$ P.M. Mockett, ${ }^{(33)}$ K.C. Moffeit, ${ }^{(25)}$ T.B. Moore ${ }^{(35)}$ M.Morii, ${ }^{(25)}$ D. Muller, ${ }^{(25)}$ T. Nagamine, ${ }^{(29)}$ S. Narita, ${ }^{(29)}$ U. Nauenberg, ${ }^{(7)}$ M. Nussbaum, ${ }^{(6)}$ N.Oishi, ${ }^{(17)}$ D. Onoprienko, ${ }^{(28)}$ L.S. Osborne, ${ }^{(16)}$ R.S. Panvini, ${ }^{(32)}$

C. H. Park, ${ }^{(27)}$ T.J. Pavel, ${ }^{(25)}$ I. Peruzzi, ${ }^{(11)}$ M. Piccolo, ${ }^{(11)}$ L. Piemontese, ${ }^{(10)}$ E. Pieroni, ${ }^{(22)}$ R.J. Plano ${ }^{(24)}$ R. Prepost, ${ }^{(34)}$ C.Y. Prescott ${ }^{(25)}$ G.D. Punkar, ${ }^{(25)}$ J. Quigley ${ }^{(16)}$

B.N. Ratcliff, ${ }^{(25)}$ J. Reidy, ${ }^{(15)}$ P.L. Reinertsen, ${ }^{\left({ }^{2}\right)}$ L.S. Rochester, ${ }^{(25)}$ P.C. Rowson, ${ }^{(25)}$ J.J. Russell, ${ }^{(25)}$ O.H. Saxton, ${ }^{(25)}$ T. Schalk, ${ }^{\left({ }^{3}\right)}$ R.H. Schindler, ${ }^{(25)}$ B.A. Schumm, ${ }^{(31)}$
J. Schwiening, ${ }^{(25)}$ S. Sen, ${ }^{(35)}$ V.V. Serbo, ${ }^{(34)}$ M.H. Shaevitz, ${ }^{(8)}$ J.T. Shank, ${ }^{(5)}$ G. Shapiro, ${ }^{(13)}$ D.J. Sherden, ${ }^{(25)}$ K. D. Shmakov, ${ }^{(28)}$ N.B. Sinev, ${ }^{(18)}$ S.R. Smith, ${ }^{(25)}$ M. B. Smy, ${ }^{(9)}$ J.A. Snyder, ${ }^{(35)}$ H. Staengle, ${ }^{(9)}$ A. Stahl, ${ }^{(25)}$ P. Stamer, ${ }^{(24)}$ R. Steiner, ${ }^{(1)}$ H. Steiner, ${ }^{(13)}$ D. $\mathrm{Su},{ }^{(25)}$ F. Suekane, ${ }^{(29)}$ A. Sugiyama, ${ }^{(17)}$ S. Suzuki, ${ }^{(17)}$ M. Swartz, ${ }^{(25)}$ F.E. Taylor, ${ }^{(16}$ J. Thom, ${ }^{(25)}$ E. Torrence, ${ }^{(16)}$ N. K. Toumbas, ${ }^{(25)}$ A.I. Trandafir, ${ }^{(14)}$ J.D. Turk, ${ }^{(35)}$

T. Usher, ${ }^{(25)}$ C. Vannini, ${ }^{(22)}$ J. Va'vra, ${ }^{(25)}$ E. Vella, ${ }^{(25)}$ J.P. Venuti, ${ }^{(32)}$ R. Verdier, ${ }^{(16)}$ P.G. Verdini, ${ }^{(22)}$ S.R. Wagner, ${ }^{(25)}$ D. L. Wagner, ${ }^{(7)}$ A.P. Waite, ${ }^{(25)}$ C. Ward, ${ }^{(4)}$

S.J. Watts, ${ }^{(4)}$ A.W. Weidemann, ${ }^{(28)}$ E. R. Weiss, ${ }^{(33)}$ J.S. Whitaker, ${ }^{(5)}$ S.L. White ${ }^{(28)}$ F.J. Wickens, ${ }^{(23)}$ D.C. Williams, ${ }^{(16)}$ S.H. Williams, ${ }^{(25)}$ S. Willocq, ${ }^{(25)}$ R.J. Wilson, ${ }^{(9)}$

W.J. Wisniewski, (25) J. L. Wittlin, ${ }^{(14)}$ M. Woods, ${ }^{(25)}$ T. R. Wright, ${ }^{(34)}$ J. Wyss, ${ }^{(20)}$ R.K. Yamamoto, ${ }^{(16)}$ X. Yang, ${ }^{(18)}$ J. Yashima, ${ }^{(29)}$ S.J. Yellin, ${ }^{(30)}$ C.C. Young, ${ }^{(25)}$ H. Yuta, ${ }^{(2)}$ G. Zapalac, ${ }^{(34)}$ R.W. Zdarko, ${ }^{(25)}$ J. Zhou. ${ }^{(18)}$

\section{(The SLD Collaboration)}

(1) Adelphi University, South Avenue- Garden City, NY 11530

(2) Aomori University, 2-3-1 Kohata, Aomori City, 030 Japan, (3) INFN Sezione di Bologna, Via Irnerio 46 I-40126 Bologna (Italy),

(4) Brunel University, Uxbridge, Middlesex - UB8 3PH United Kingdom,

${ }^{(5)}$ Boston University, 590 Commonwealth Ave. - Boston, MA 02215, (6) University of Cincinnati, Cincinnati, $\mathrm{OH} 45221$

(7) University of Colorado, Campus Box 390 - Boulder, CO 80309

${ }^{(8)}$ Columbia University, Nevis Laboratories P.O.Box 137 - Irvington, NY 10533, ${ }^{(9)}$ Colorado State University, Ft. Collins, CO 80523,

(10) INFN Sezione di Ferrara, Via Paradiso,12 - I-44100 Ferrara (Italy),

${ }^{(11)}$ Lab. Nazionali di Frascati, Casella Postale 13 I-00044 Frascati (Italy)

(12) University of Illinois, 1110 West Green St. Urbana,IL 61801

${ }^{(13)}$ Lawrence Berkeley Laboratory, Dept.of Physics 50B-5211 University of CaliforniaBerkeley, $C A 94720$

(14) University of Massachusetts, Amherst,MA 01003,

(15) University of Mississippi, University, MS 38677 ,

(16) Massachusetts Institute of Technology, 77 Massachussetts Avenue Cambridge, MA 02139

(17) Nagoya University, Nagoya 464 Japan,

${ }^{(18)}$ University of Oregon, Department of Physics Eugene, OR 97403,

(19) Oxford University, Oxford, OX1 3RH, United Kingdom,

(20) Universita di Padova, Via F. Marzolo,8 I-35100 Padova (Italy),

(21) Universita di Perugia, Sezione INFN, Via A. Pascoli I-06100 Perugia (Italy), (22) INFN, Sezione di Pisa, Via Livornese,582/AS Piero a Grado I-56010 Pisa (Italy), ${ }^{(23)}$ Rutherford Appleton Laboratory, Chiton,Didcot - Oxon OX11 OQX United Kingdom, 
(24) Rutgers University, Serin Physics Labs Piscataway, NJ 08855-0849,

${ }^{(25)}$ Stanford Linear Accelerator Center, 2575 Sand Hill Road Menlo Park, CA 94025, (26) Sogang University, Ricci Hall Seoul, Korea,

(27) Soongsil University, Dongjakgu Sangdo 5 dong 1-1 Seoul, Korea 156-743,

(28) University of Tennessee, 401 A.H. Nielsen Physics Blg. - Knoxville, Tennessee 37996-1200,

(29) Tohoku University, Bubble Chamber Lab - Aramaki - Sendai 980 (Japan),

${ }^{(30)}$ U.C. Santa Barbara, 3019 Broida Hall Santa Barbara,CA 93106 ${ }^{(31)}$ U.C. Santa Cruz, Santa Cruz, CA 95064,

(32) Vanderbilt University, Stevenson Center,Room 5333 P.O.Box 1807.Station B Nashville, TN 37935

(33) University of Washington, Seattle, WA 98105 ,

(34) University of Wisconsin, 1150 University Avenue Madison,WS 53706

${ }^{(35)}$ Yale University, 5th Floor Gibbs Lab. - P.O.Box 208121 - New Haven,CT 06520-8121.

\section{Bibliography}

[1] SLD Collab., K. Abe et al., Phys. Rev. Lett. 73 (1994) 25. LEP Collaborations and LEP Electroweak Working Group, CERN-PPE-93-157 (1993).

[2] For a recent review of $\alpha_{s}$ measurements in $e^{+} e^{-}$annihilation see: P.N. Burrows, 'Results on $\alpha_{s}$ and QCD from (and Above) the $Z^{0}$, SLAC-PUB-7631; to appear in Proceedings of the XVII International Conference on Physics in Collision, Bristol, England, June 25-27 1997.

[3] M. Bilenky, G. Rodrigo, A. Santamaria, Nucl. Phys. B439 (1995) 505; J. Fuster, S. Cabrera, S. Marti i Garcia, Nucl. Phys. (Proc. Suppl.) 54A (1997) 39.

[4] T. G. Rizzo, Phys. Rev. D50 4478 (1994).

[5] TASSO Collaboration: W. Braunschweig et al., Z. Phys. C42 17 (1989); W. Braunschweig et al., Z. Phys. C44 365 (1989).

[6] DELPHI Collaboration: P. Abreu et al., Phys. Lett. B307 221 (1993); L3 Collaboration: B. Adeva et al., Phys. Lett. B271 461 (1991); OPAL Collaboration: M.Z. Akrawy et al., Z. Phys. C65 31 (1995); ALEPH Collaboration: D. Buskulic et al., Phys. Lett. B355 381 (1995).

[7] OPAL Collaboration: R. Akers et al., Z. Phys. C60 397 (1993).

[8] SLD Design Report, SLAC Report-273 (1984), unpublished.

[9] SLD Collaboration: K. Abe et al., Phys. Rev. D53 2271 (1996).

[10] M. Gell-Mann, Phys. Lett. 8214 (1964);

G. Zweig, CERN-TH-412 (1964). 
[11] M. Y. Han, Y. Nambu, Phys. Rev. B139, 1006 (1965).

[12] E. Bloom et al., Phys. Rev. Lett. 23, 930 (1969); M. Breidenbach et al., Phys. Rev. Lett. 23, 935 (1969).

[13] J. D. Bjorken, Phys. Rev. 179, 1547 (1969); J. D. Bjorken, Phys. Rev. D8, 4098 (1973).

[14] Mark I Collaboration: G. Hanson et al., Phys. Rev. Lett. 35, 1320 (1975).

[15] Mark I Collaboration: R. F. Schwitters et al., Phys. Rev. Lett. 35, 1609 (1975); TASSO Collaboration: M. Althoff et al., Z. Phys. C22, 307 (1984).

[16] TASSO Collaboration: R. Brandelik et al., Phys. Lett. B86, 243 (1979); MARK J Collaboration: D. P. Barber et al., Phys. Rev. Lett. 43, 830 (1979); PLUTO Collaboration: C. Berger et al., Phys. Lett. B86, 418 (1979); JADE Collaboration: W. Bartel et al., Phys. Let. B91, 142 (1980).

[17] The Particle Data Group., http://www-pdg.lbl.gov/1997.

[18] S. Weinberg, Phys. Rev. Lett. 19, 1264 (1967);

A. Salam in Elementary Particle Theory, ed. N. Svartholm(Almquist and Wiksells, Stockholm, 1969) p. 367

S. L. Glashow, J. Iliopoulos, and L. Maiani, Phys. Rev. D2, 1285 (1970).

[19] N. Cabibbo, Phys. Rev. Lett. 10, 531 (1963);

M. Kobayashi and T. Masukawa, Prog. Theor. Phys. 49, 652 (1973).

[20] The Particle Data Group., Phys. Rev. D51 (1996).

[21] S. Fanchiotti, B. Kniehl, and A. Serlin, Phys. Rev. D48 307 (1993) and references therein.

[22] W. J. Marciano and A. Sirlin, Phys. Rev. Lett. 61, 1815 (1988).

[23] The SLD Collaboration: K. Abe et al., Phys. Rev. Lett. 7325 (1994).

[24] C. N. Yang, R. L. Mills, Phys. Rev. 96191 (1954).

[25] G. 't Hooft, Nucl. Phys. 33173 (1971).

[26] J.Schwinger, Phys. Rev. 73, 416 (1948) S.Tomonaga, Phys. Rev. 74, 224 (1948) F. J. Dyson, Phys. Rev. 75, 486 (1949).

[27] G. Kramer, Theory of Jets in Electron Positron Annihilation, Springer Tracts of Modern Physics, Vol 102, Springer (1984).

[28] The Particle Data Group., Phys. Rev. D51 (1996) Chapter 9. Quantum Chromodynamics.

[29] M. Dine, J. Sapirstein, Phys. Rev. Lett. 43, 668 (1979).

[30] G. 't Hooft, M. Veltman, Nucl. Phys. B44 189 (1972); C. G. Bollini, J. J. Giambiagi, Nuovo Cim. 12B 20 (1972);

J. F. Ashmore, Lett. Nuovo Cim. 4289 (1972);

G. M. Cicuta, E. Montaldi, Lett. Nuovo Cim. 4329 (1972).

[31] SLD Collaboration: K. Abe et al., Phys. Rev. D51 962 (1995).

[32] P. N. Burrows, Acta Phys. Polon. B28 701 (1997).

[33] S. Brandt et al., Phys. Lett. 12, 57 (1964); E. Farhi, Phys. Rev. Lett. 39, 1587 (1977)

[34] L. Clavelli, Phys. Lett. B85, 111 (1979).

[35] S. Catani, G. Turnock and B. R. Webber, Phys, Lett. B295, 269 (1992).

[36] MARK-J Collab., D. P. Barber et al., Phys. Rev. Lett. 43, 830 (1979); Phys. Lett. B89, 139 (1979).

[37] G. Parisi, Phys. Lett. B74, 65 (1978); J. F. Donoghue, F. E. Low, S. Y. Pi, Phys.Rev. D20, 2759 (1979).

[38] C. L. Basham et. al., Phys. Rev. Lett. 41, 1585 (1978); Phys. Rev. D17, 2298 (1978); Phys. Rev. D19, 2018 (1979). 
[39] T. Cheng and L. Li, Gauge theory of elementary particle physics, Chapter 9.3, Oxford University Press (1984).

[40] J. C. Taylor, Nucl. Phys. B33 436 (1971).

[41] Applications of Perturbative QCD, By R. D. Field, Addison-Wesley, (1989), Chapter

[42] P. N. Burrows, Precise Tests of QCD in $e^{+} e^{-}$Annihilation, Proceedings of the XXIV SLAC Summer Institute on Particle Physics, SLAC-R-508.

[43] K. G. Wilson, Phys. Rev. D10, 2445 (1997);

T. DeGrand, Lattice Gauge Theory for QCD, Proceedings of the XXIV SLAC Summer Institute on Particle Physics, SLAC-R-508.

[44] Y. I. Azimov, Y. L. Dokshitser, V. A. Khoze, S. I. Troian, Z. Phys. C27 65 (1985).

[45] J. J. Hernandez et al., Phys. Lett. 2391 (1990).

[46] M. Schmelling, Phys. Scripta 51683 (1995).

[47] G. Altarelli and G. Parisi, Nucl. Phys. B126 298 (1977).

[48] Z. Kunszt, P. Nason, G. Marchesimi, and B. R. Webber, Proceedings of the Workshop on $Z$ Physics at LEP I;

G. Altarelli, R. Kleiss, and C. Verzegnassi, CERN-89-08 p. 414 vol1.

[49] G. Kramer and B. Lampe, Z. Phys. C42 504 (1989).

[50] S. G. Gorishny, A. Kataev, and S. A. Larin, Phys, Lett. B259, 144 (1991); L. R. Surguladze and M. A. Samuel, Phys. Rev. Lett. 66, 560 (1991).

[51] F. Le Diberder and A. Pich, Phys. Lett. B289, 165 (1992); E. Braaten, S. Narison, and A. Pich, Nucl. Phys. B373, 581 (1992);

F. Le Diberder and A. Pich, Phys. Lett. B286, 147 (1992);

M. Neubert, Nucl. Phys. B463, 511 (1996).
[52] S. Catani, G. Turnock, B.R. Webber, Phys. Lett. B263 491 (1991); S. Catani, G. Turnock, B.R. Webber, Phys. Lett. B272 368 (1991)

S. Catani, Yu.L. Dokshitzer, M. Olsson, G. Turnock, B.R. Webber, Phys. Lett. B269 432 (1991);

S. Catani, L. Trentadue, G. Turnock, B.R. Webber, Nucl. Phys. B407 3 (1993).

[53] V. N. Gribov and I. N. Lipatov, Sov. J. Nucl. Phys. 15438 (1972);

Yu. L. Dokshitzer, D. I. D'yakonov and S. I. Troyan, Proceedings of the 13th Winter School of LNPI Leningrad (1978), SLAC-TRANS-183(1978).

[54] X. Artru and G. Mennessier, Nucl. Phys. B70 93 (1974).

[55] B. Anderson and G. Gustafson, G. Ingelman and T. Sjöstrand, Phys. Rep. 9731 (1983).

[56] T. Sjöstrand, Comput. Phys. Commun. 43367 (1987).

[57] G. Marchesini et al., Comp. Phys. Comm. 67, 465 (1992).

[58] D. Amati, G. Veneziano, Phys. Lett. 83B 87 (1979).

[59] R. D. Field and R. P. Feynman, Nucl. Phys. B136, 1 (1978)

[60] JADE Collaboration: W. Bartel et al., Phys. Lett. B101 129 (1981); L3 Collaboration: M. Acciarri, et al., Phys. Lett. B345 74 (1995); DELPHI Collaboration: P. Abreu, et al., Z. Phys., C70 179, (1996); OPAL Collaboration: CERN-PPE/95-83.

[61] J. D. Bjorken, Phys. Rev. D17, 171 (1978).

[62] M. G. Bowler, Z. Phys. C11 169 (1981).

[63] C. Peterson, et. al, Phys. Rev. D27 105 (1983).

[64] ALEPH Collaboration: D. Buskulic et al., Phys. Lett. B357 699 (1995); ALEPH Collaboration: D. Buskulic et al., Z. Phys. C62 179 (1994); DEPHI Collaboration: P. Abreu et al., CERN PPE/95-08; 
OPAL Collaboration: G. Alexander et al., Phys. Lett. B364 93 (1995); OPAL Collaboration: R. Akers et al., Z. Phys. C60 199 (1993).

[65] ALEPH Collaboration: D. Buskulic et al., Z. Phys. C62 179 (1994); OPAL Collaboration: G. Alexander et al., CERN PPE/96-051.

[66] SLD Collaboration: K. Abe et al., Phys.Rev. D56, 5310 (1997).

[67] L3 Collaboration: B. Adeva et al., Phys. Lett. B271 461 (1991).

[68] JADE Collab., W. Bartel et al., Z. Phys. C33 (1986) 23.

[69] DELPHI Collaboration: P. Abreu et al., Phys. Lett. B307 221 (1993).

[70] S. Bethke et al., Nucl. Phys. B370 (1992) 310 .

[71] DELPHI Collaboration: P. Abreu et al., CERN-PPE-97-141, to appear in Phys. Lett. B.

[72] ALEPH Collaboration: D. Buskulic et al., Phys. Lett. B355 381 (1995).

[73] OPAL Collaboration: M.Z. Akrawy et al., Z. Phys. C65 31 (1995); ALEPH Collaboration: D. Buskulic et al., Phys. Lett. B355 381 (1995).

[74] P. Dervan, Ph.D. Thesis, Brunel University.

[75] M. Breidenbach, SLC and SLD - Experimental Experience with a Linear Collider, in Waikoloa 1993, Proceedings, Physics and Experiments with Linear $e+e^{-}$Colliders, vol. 1, p. 30-45; SLAC-PUB-6313.

[76] The SLD Collaboration, http://www-sld.slac.stanford.edu/.

[77] F. J. Decker, R. Holtzapple, T. Raubenheimer, Over Compression, a Method to Shape the Longitudinal Bunch Distribution for a Reduced Energy Spread, SLAC-PUB-6604, Presented at 17th International Linear Accelerator Conference (LINAC 94), Tsukuba, Japan, 21-26 Aug 1994. Published in Tsukuba LINAC 1994:47-49.

[78] M. D. Hildreth et al., Nucl. Instr. Meth. A367 111 (1995).
[79] C. J. S. Damerell et al., Nucl. Instr. Meth. A275 484 (1989);

C. J. S. Damerell et al., Nucl. Instr. Meth. A288 236 (1990);

G. D. Agnew et al. Design and Performance of the SLD Vertex Detector, a 120M Pixel Tracking System in Proceedings of the 26th International Conference on High Energy Physics, Dallas Vol2 1862 (1992).

[80] K. Abe et. al., Nucl. Instr. Meth. A343 74 (1994).

[81] D. Axen et. al., Nucl. Instr. Meth. A328 472 (1993).

[82] C. J. S. Damerell et al. IEEE Trans. on Nucl. Sci., 37305 (1990).

[83] SLD Collaboration: K. Abe et al., Phys. Rev. D53 1023 (1996).

[84] J. P. Venuti et al. IEEE Trans. Nucl. Sci., 36595 (1989).

[85] J. T. Walker et al. "Microstore - the Stanford Analog Memory Unit", IEEE Trans. on Nucl. Sci., 32616 (1985).

[86] T. Pavel, Ph. D Dissertation, Stanford University, SLAC-R-491 (1996).

[87] A. C. Benvenuti et. al., Nucl. Instr. Meth. A276 95 (1989).

[88] S. Berridge et al., IEEE Trans. Nucl. Sci. 371191 (1990); K. T. Pitts, Ph. D. Thesis, SLAC-0446, (1994).

[89] P. N. Burrows, H. Masuda, An optimized QCD Parameter Set for the JETSET 7.4 Monte Carlo Program, SLD Physics Note 36 (1995).

[90] CLEO B decay model provided by P. Kim and the CLEO Collaboration.

[91] B. Barish et al., Phys. Rev. Lett. 761570 (1996);

H. Albrecht et al., Z. Phys. C58 191 (1993);

H. Albrecht et al., Z. Phys. C62 371 (1994);

F. Muheim, in Proceedings of the 8th Meeting Division of Particles and Fields, Albuquerque, August, 1994 (World Scientific, Singapore, 1995), Vol. 1, p.851;

M. Thuladidas, Ph.D thesis, Syracuse University, 1993; 
G. Grawford et al., Phys. Rev. D45 752 (1992);

D. Bortoletto et al., Phys. Rev. D45 21 (1992).

[92] T. Junk, Ph.D. Thesis, Stanford University, Appendix E, SLAC-REPORT-95-467 (1995).

[93] R. Brun et al., CERN-DD/EE/84-1 (1989).

[94] P. Billoir, Nucl. Instrum. Meth. A225, 352 (1984).

[95] SLD Collaboration, Design and Performance of the SLD Vertex Detector, a $307 \mathrm{M}$ pixel Tracking System, submitted to Nucl. Instr. Meth. slac-pub-7385.

[96] M. G. Strauss, Performance of the SLD CCD pixel vertex detector and design of an upgrade, Proceedings of 1994 ICHEP, Glasgow, 1179-1184.

[97] H. A. Neal Jr.II, Measurements of $\Gamma\left(Z^{0} \rightarrow b \bar{b}\right) / \Gamma\left(Z^{0} \rightarrow\right.$ Hadrons $)$ using the SLD., Ph.D. Thesis, Stanford, (1995).

[98] D. Bourilkov et al. Nucl. Instr. Meth. A394 103 (1997).

[99] K. G. Baird, III, Ph.D. Thesis, Rutgers U., SLAC-R-0483, (1996).

[100] D. Jackson, Nucl. Instrum. Methods A388, 247, 1997.

[101] J. M. Yamartino, A measurement of the $e^{+} e^{-}$Decay Width of the $Z^{0} \mathrm{Ph}$. D Thesis, MIT, (1994) SLAC-Report-426 Chapter 4;

J. M. Yamartino, Hadronic Event Selection Using the LAC, SLD Physics Note 14 (1993).

[102] D. Williams, The Left-right Forward - backward Asymmetry for B Quarks at the SLD, Ph.D. Thesis, SLAC (1994) SLAC-0445, Chapter 4

[103] P. N. Burrows et al. Estimate of Combined Triggering and Selection Efficiency for Hadronic Events, SLD internal note 229, unpublished.

[104] TASSO Collaboration: W. Braunschweig et al., Z. Phys. C38 543 (1988).

[105] MARK-II Collaboration: R. Jacobsen et al., Phys. Rev. Lett. 673347 (1991).
[106] V. D. Barger, R. J. N. Phillips Collider Physics, Addison-Wesley (1987).

[107] P. N. Burrows, D. Muller, N. Oishi SLD Physics Note 67 (unpublished).

[108] For example;

Probability and Statistics in Particle Physics, By A.G. Frodesen, O. Skjeggestad, H. Tofte. Universitetsforlaget (1979).

[109] J. Zhou, Studies of Multiparticle Production in Hadronic $Z^{0}$ Decays, Ph.D. Thesis, University of Oregon (1996), SLAC-R-496, Chapter 5.

[110] SLD Collaboration: K. Abe et al., to appear in Phys. Rev. Lett.

[111] V. V. Serbo, Measurement of the Polarized Forward - Backward Asymmetry of b quark at SLD. Ph.D Thesis, Wisconsin U., Madison (1997), SLAC-R-0510, Chapter 4.

[112] The LEP Electroweak Working Group, Presentation of LEP Electroweak Heavy Flavor Results for Summer 1996 Conferences LEPHF/96-01 (1996).

[113] SLD Collaboration: K. Abe et al., Phys. Lett. B386 475 (1996).

[114] OPAL Collaboration: R. Akers et al., Phys. Lett. B352 176 (1995) DEPHI Collaboration: P. Abreu et al., Phys. Lett. B347 447 (1995).

[115] ARGUS Collaboration: H. Albrecht et al., Z. Phys. C54 13 (1992).

[116] MARK-III Collaboration: D. Coffman et al., Phys. Lett. B263 135 (1991).

[117] OPAL Collaboration: R. Akers et al., Phys. Lett. B353 595 (1995); OPAL Collaboration: R. Akers et al., Z. Phys. C67 27 (1995).

[118] I. G. Knowles et al. 1996 Physics at LEP 2 vol 2 yellow report CERN 96-01 p.112, Table 2.

[119] K. Abe et al. Phys. Rev. Lett. 783442 (1997).

[120] I. G. Knowles, G. D. Lafferty, J. Phys. G23 731 (1997).

[121] A. Brandenburg, private communications. 
[122] S. Bethke et al., Nucl. Phys. B370 310 (1992).

[123] Z. Kunszt et al., CERN 89-08 Vol. I, 373 (1989).

[124] B. L. Ioffe et al., Phys. Lett. B78 277 (1978).

[125] A. Ballestrero, E. Maina, S. Moretti, Phys. Lett. B294 425 (1992).

[126] A. Ballestrero, E. Maina, S. Moretti, Nucl. Phys. B415 265 (1994)

[127] G. Rodrigo, A. Santamaria, M. Bilenky, Phys. Rev. Lett. 79193 (1997).

[128] W. Bernreuther, A. Brandenburg, P. Uwer, Phys. Rev. Lett. 79, 189 (1997) A. Brandenburg, P. Uwer, Aachen preprint PITHA-97-29, hep-ph/9708350.

[129] C. A. Dominguez, N. Paver, Phys. Lett. B293, 197 (1992);

S. Narison, Phys. Lett. B341, 73 (1994);

M. Neubert, Phys. Rept. 245, 259 (1994);

S. Titard, F. J. Yndurain, Phys. Rev. D49 6007 (1994);

M. Crisafulli, V. Gimenez, G. Martinelli, C. T. Sachrajda, Nucl.Phys. B457, 594 (1995);

V. Gimenez, G. Martinelli, C. T. Sachrajda, Phys. Lett. B393, 124 (1997);

C. T. H. Davies et al. Phys.Lett. B345, 42 (1995).

[130] L. Dixon, A. Signer, Phys. Lev. Lett 78811 (1997)

L. Dixon, A. Signer, SLAC-PUB-7528 (1997); submitted to Phys. Rev. D.

[131] L. Dixon, private communications. 